This item was submitted to Loughborough's Research Repository by the author.

Items in Figshare are protected by copyright, with all rights reserved, unless otherwise indicated.

\title{
Compressed sensing current mapping spatial characterization of photovoltaic devices
}

\section{PLEASE CITE THE PUBLISHED VERSION}

\section{PUBLISHER}

(c) George Koutsourakis

\section{PUBLISHER STATEMENT}

This work is made available according to the conditions of the Creative Commons Attribution-NonCommercialNoDerivatives 4.0 International (CC BY-NC-ND 4.0) licence. Full details of this licence are available at: https://creativecommons.org/licenses/by-nc-nd/4.0/

\section{LICENCE}

CC BY-NC-ND 4.0

\section{REPOSITORY RECORD}

Koutsourakis, George. 2019. "Compressed Sensing Current Mapping Spatial Characterization of Photovoltaic Devices”. figshare. https://hdl.handle.net/2134/25750. 


\title{
Compressed Sensing Current
}

\section{Mapping Spatial Characterization of Photovoltaic Devices}

\author{
George Koutsourakis
}

\author{
Doctoral Thesis
}

May 2017 


\section{Al Loughborough University}

\section{CERTIFICATE OF ORIGINALITY}

This is to certify that I am responsible for the work submitted in this thesis, that the original work is my own except as specified in acknowledgments or in footnotes, and that neither the thesis nor the original work contained therein has been submitted to this or any other institution for a degree. 


\section{Abstract}

In this work a new measurement technique for current mapping of photovoltaic (PV) devices is developed, utilising the compressed sensing (CS) sampling theory. Conventional current mapping measurements of PV devices are realised using the light beam induced current (LBIC) measurement method. For its realization, a light beam scans a PV device and the induced current is measured for every point, generating the final current map of the device. Disadvantages of the LBIC method are the low measurement speed, the complicated and usually expensive measurement layouts and the impractical application of the method on PV modules. With the development of CS current mapping in this work, the above issues can be mitigated. Instead of applying a raster scan, a series of illumination patterns are projected onto the PV sample, acquiring fewer measurements than the pixels of the final current map. The final reconstruction of the current map is achieved by means of an optimisation algorithm.

Spatially resolved electrical simulations of CS current mapping demonstrate that theoretically the proposed method is feasible. In addition, it is shown that current maps can be acquired with even $40 \%$ of the measurements a standard LBIC system would require, saving a significant amount of measurement time. The performance of CS current mapping is the same, regardless of the features a sample may contain and measurements can be applied to any type of photovoltaic device. The ability of the method to provide current maps of PV modules is demonstrated. The performance of several reconstruction algorithms is also investigated.

An optical measurement setup for CS current mapping of small area PV devices was built at the National Physical Laboratory (NPL), based on a digital micromirror device (DMD). Accurate current maps can be produced with only $40 \%$ of the measurements a conventional point by point scan would need, confirming simulation results. The measurement setup is compact, straightforward to realise and uses a small number of optical elements. It can measure a small area of $1 \mathrm{~cm}$ by $1 \mathrm{~cm}$, making it ideal for current mapping of small research samples. A significant signal amplification is achieved, since the patterns illuminate half of the sample. This diminishes the use of lock-in techniques, reducing the cost for current mapping of PV devices. Current maps of an optical resolution up to $27 \mu \mathrm{m}$ are acquired, without the use of any demagnification elements of the projected pattern that the DMD generates. 
A scale up of this new current mapping method is demonstrated using Digital Light Processing (DLP) technology, which is based on DMD chips. A commercial DLP projector is utilised for building a proof of concept CS current mapping measurement system at the Centre of Renewable Energy Systems Technology (CREST). Current maps of individual PV cells in encapsulated modules can be acquired, something that is extremely difficult to achieve with conventional LBIC systems. Direct current mapping of a PV module with by-pass diodes is successfully applied for the first time. Specific shading strategies are developed for this purpose in order to isolate the cell under test. Due to the application of compressive sampling, current maps are acquired even if the signal-to-noise-ratio levels are so low that a point by point scan is not possible.

Through the above implementations of CS current mapping of this work, the proposed technique is studied and evaluated. The results demonstrate that this novel method can offer a realistic alternative approach for current mapping of PV cells and modules that can be cost effective and straightforward to implement. In addition, this work introduces the application of the CS theory and DLP technology to PV metrology in general. 


\section{Acknowledgments}

First, I would like to thank my supervisors Professor Ralph Gottschalg and Dr Thomas Betts, for giving me the chance to work in the APV group, their invaluable support and guidance throughout my PhD studies and for their confidence in my abilities to carry out this work.

I would also like to specially thank my NPL supervisor Simon Hall for his supervision, support and help during my periods working at NPL and specifically for transferring me his passion and vision about this work.

I would like to thank Dr Martin Bliss and Dr Matt Cashmore, for their technical and scientific support at CREST and at NPL respectively. Special thanks also go to Dr Xiaofeng Wu, for our perfect collaboration and research work that I really enjoyed.

For their support, special thanks are due to all current and previous members of the Applied Photovoltaics group in CREST that I have worked with and collaborated, for their help, feedback and support whenever I needed it. Special thanks should also go to my friends at the office at CREST, Michael Owen-Bellini, Farhad Anvari-Azar, Dr lan Cole and Dr Karl Bedrich, we had some great fun together during my PhD period, mostly during work times.

Many thanks have to go to my parents and my brother, for their encouragement and support throughout my PhD studies.

Above all, I would like to thank my better half Elena Koumpli, for her constant support, encouragement and for helping me concentrate in order to deliver this work. 


\section{Table of Contents}

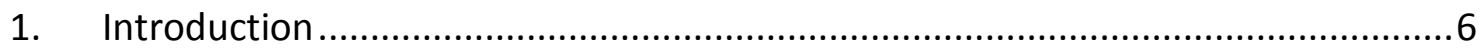

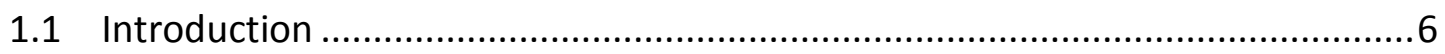

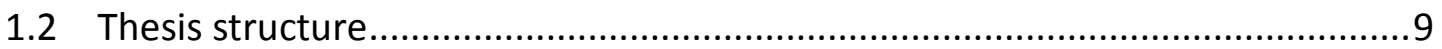

2. Solar Cell Operation and Spatial Characterisation Techniques...........................12

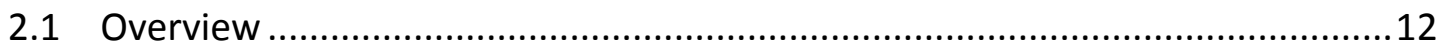

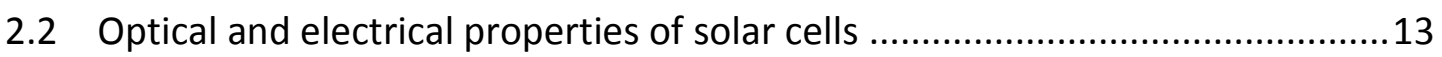

2.2.1 Semiconductors and optical absorption................................................13

2.2.2 P-n junction and PV device operation ...................................................15

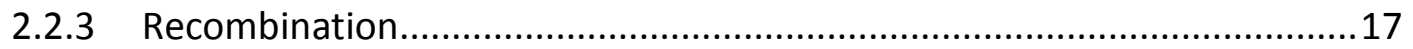

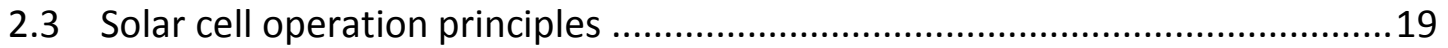

2.4 Spatial features affecting the performance of PV devices ...............................22

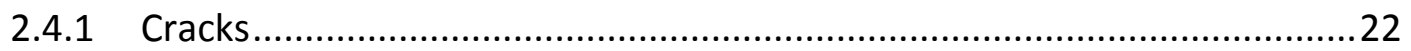

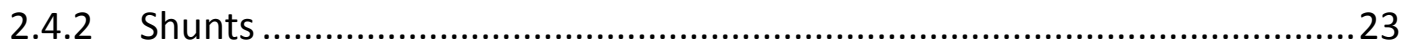

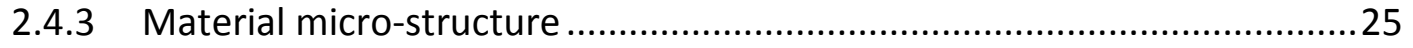

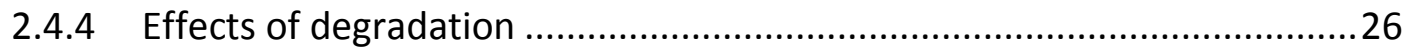

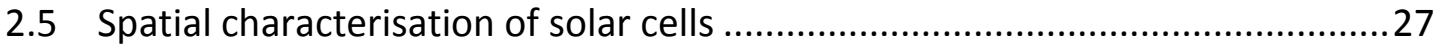

2.5.1 Light beam induced current (LBIC) measurements .................................22

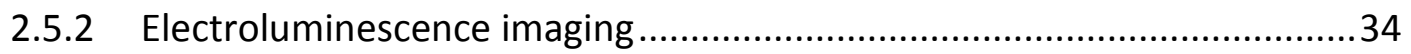

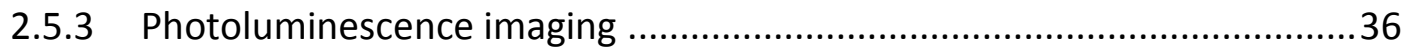

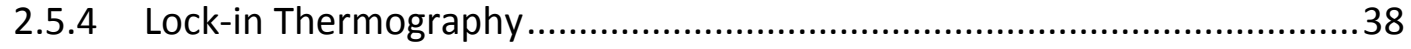

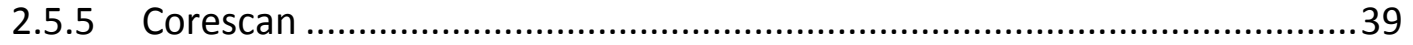

2.5.6 Electron Beam Induced Current (EBIC) measurements ............................40

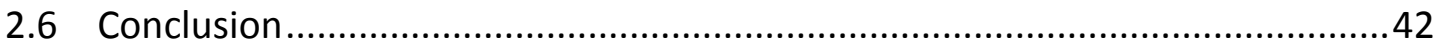

3. Compressed sensing imaging and application to PV spatial characterisation ......44

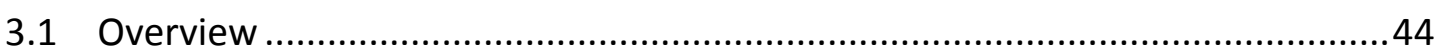

3.2 The Digital Micromirror Device (DMD) …................................................... 46

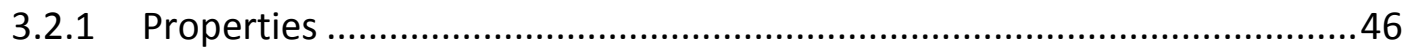

3.2.2 Application to PV characterisation ................................................... 48

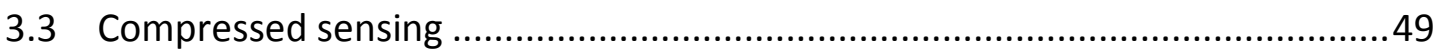

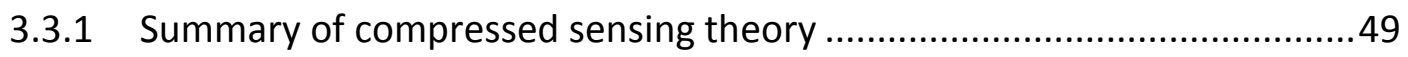

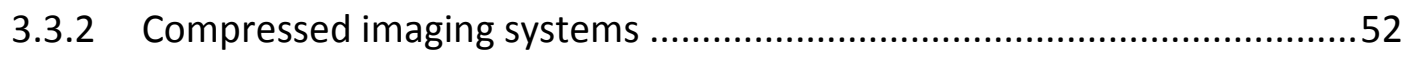

3.3.3 Applications in spatial characterisation instrumentation ........................54 
3.4 Applying compressed sensing to PV current mapping .................................55

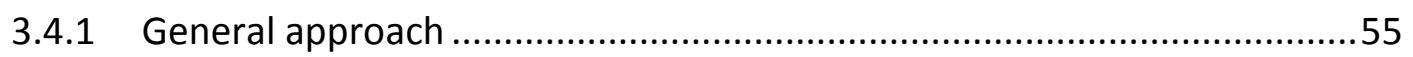

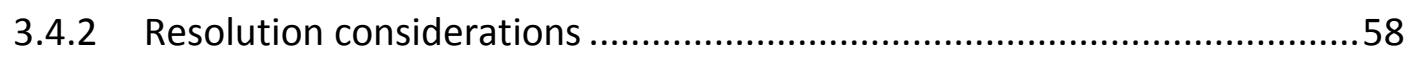

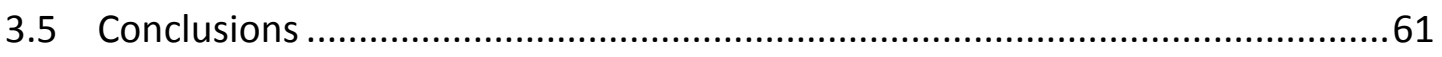

4. Spatially Resolved Electrical Simulations of Compressed Sensing Current Mapping 64

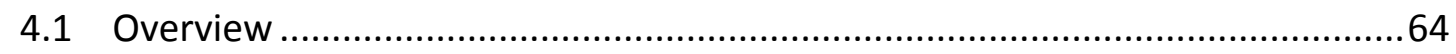

4.2 Compressive Sampling Using the PVONA toolset..........................................65

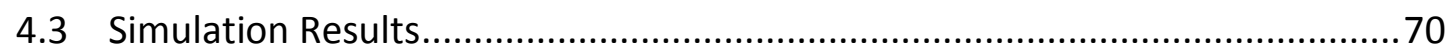

4.3.1 Simulated Crystalline Silicon PV samples ...............................................70

4.3.2 Simulated Thin Film PV Devices.............................................................. 75

4.4 Comparison of Reconstruction Algorithms.................................................... 79

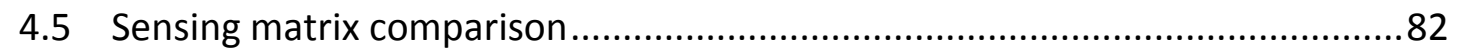

4.6 Signal levels, dynamic range and noise considerations...................................83

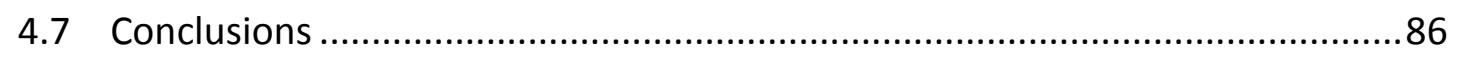

5. Small Area Compressed Sensing Current Mapping System ................................89

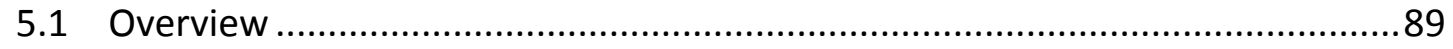

5.2 Initial CS Current Mapping System ...........................................................91

5.2.1 Optical system design .......................................................................91

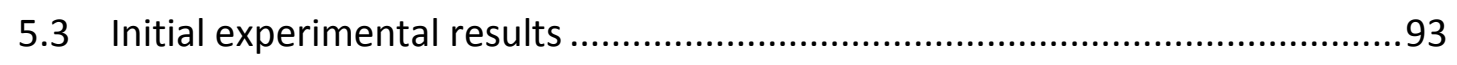

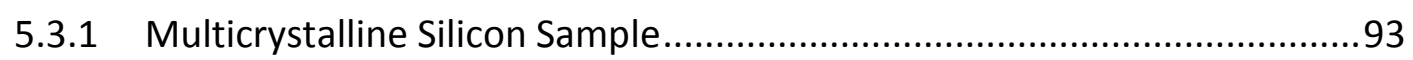

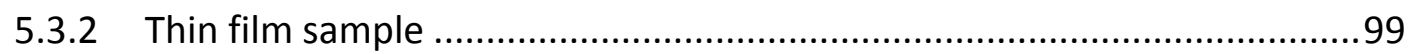

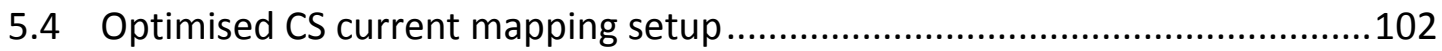

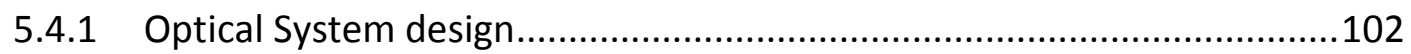

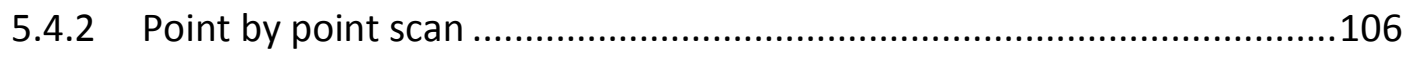

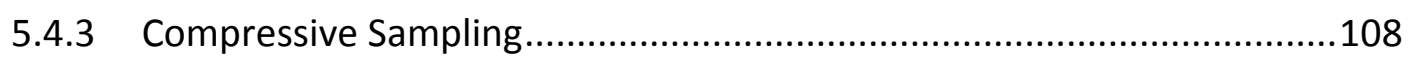

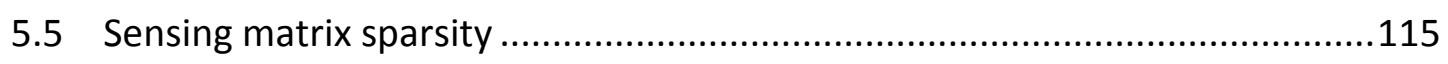

5.6 Practical Considerations and Discussion ..................................................... 121

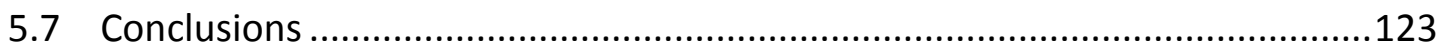

6. Scalability of Compressed Sensing Current Mapping........................................126

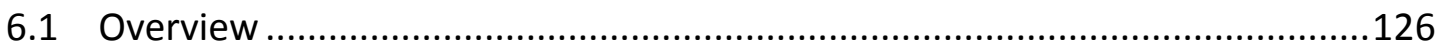

6.2 Operating principles of a Digital Light Processing (DLP) projector.................128

6.3 Evaluation of the DLP projector as a light source for current mapping ..........129

6.3.1 Projector features and initial tests ....................................................129 
6.3.2 Projector unit modifications

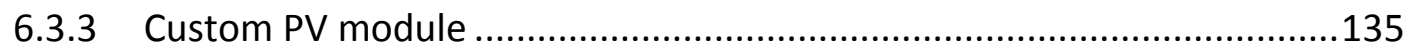

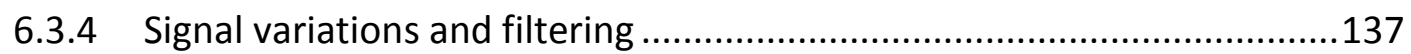

6.3.5 Experimental setup and procedure for CS current mapping ..................139

6.4 CS current mapping measurements ….......................................................141

6.4.1 Current mapping results for individual, large area cells .........................141

6.4.2 Voltage bias considerations for current mapping of modules .................144

6.4.3 Current mapping results for PV modules of interconnected cells ...........147

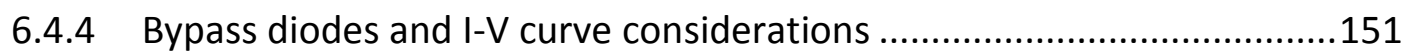

6.4.5 Practical Considerations and Discussion ............................................157

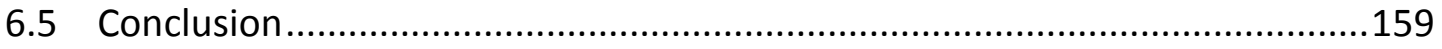

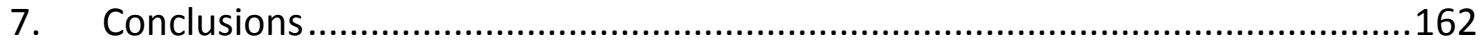

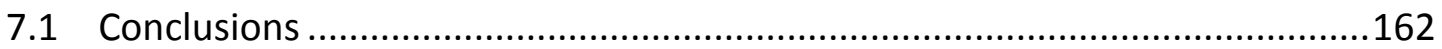

7.1.1 Spatially Resolved Electrical Simulations of Compressed Sensing Current Mapping 163

7.1.2 Small Area Compressed Sensing Current Mapping system .....................164

7.1.3 Scalability Compressed Sensing Current Mapping ..................................165

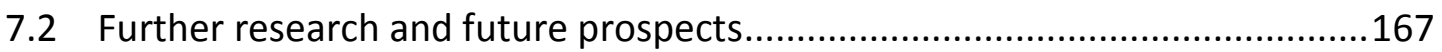

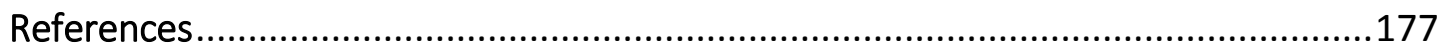




\section{Chapter 1}

\section{Introduction}

\subsection{Introduction}

Photovoltaic (PV) electricity generation currently covers $1 \%$ of global electricity demand, with optimistic future scenarios of PV penetration into the energy mix increasing this to $16 \%$ by 2050 [1]. In order to achieve this, highly efficient, stable and cost-effective products need to be supplied to the market to compete with other power generation technologies. To realise this aim, more research has to be undertaken into existing and new materials for PV applications, to increase efficiencies and long term durability. For a specific output power, higher cell efficiency directly results in a smaller and therefore less expensive PV system, provided that costs do not also rise. For established technologies, optimised production methods have led to both higher efficiencies and cost reduction of production processes. These gains are closely linked to commercial transfer of research knowledge and innovation in the PV field [2]. Optimising current PV production technologies and developing new ones will keep pushing PV product prices to even lower levels [3].

Crystalline silicon PV modules currently dominate the market, with the record efficiency for single junction mono crystalline cells reaching recently a $26.3 \%$ [4], while for multicrystalline cells the record is set at a lower efficiency at $21.9 \%$ [5]. Thin film technologies have also achieved high efficiencies recently with $\mathrm{Cu}(\mathrm{In}, \mathrm{Ga}) \mathrm{Se} 2$ (CIGS) solar cells reaching a 
record efficiency of 22.6\% [6] and CdTe cells achieving an efficiency of 22.1\% [7]. Emerging technologies such as perovskite solar cells also exhibit respectably high efficiencies. However, the issue of long term stability for such technologies has not yet been resolved. Still for all the current technologies of solar cells, there is further room for improvement on efficiency to approach the Shockley-Queisser limit [8].

For a better understanding of the impact of material properties, cell design, cell structure and ageing effects on solar cell performance, PV device characterisation methods are extremely crucial. A large number of PV characterisation techniques are available nowadays, from cell to module level. Different techniques are useful for different kinds of samples, providing a large range of parameters of devices. The most well-known standard method for characterising PV devices is by using a solar simulator to acquire the currentvoltage characteristics and derived performance parameters such as the power at maximum power point. Such measurements are most commonly carried out under standard test conditions (STC). These conditions are device temperature of $25{ }^{\circ} \mathrm{C}$, a light spectrum corresponding to air mass of a value of $1.5,1000 \mathrm{~W} / \mathrm{m}^{2}$ light intensity and normal incidence. This power rating provides a fast and simple indication of how a device performs under specific standard conditions and more importantly it sets a reference for comparison between different devices and technologies.

Confirming that a cell is underperforming by acquiring the current-voltage characteristic curve does not necessarily reveal the reason of this reduction of energy output. Local defects that can be induced during production or after degradation may have a significant impact on performance. For a more in depth investigation of the features of solar cells that affect performance, spatial characterisation of devices is necessary. Non-invasively assessing local performance can provide useful information for many properties of PV devices: material and structural properties of solar cells can be acquired, as well as local optical and electronic properties. Spatial characterisation is important in all steps of PV product development and manufacturing, from small research cells to commercial-scale modules. Various methods have been developed over the years from nanometre to millimetre resolution regions to accommodate for all types and sizes of samples. The most well-established non-destructive spatial characterisation methods are luminescence imaging methods such as electroluminescence [9] and photoluminescence [10], lock-in thermography [11] and light beam induced current mapping (LBIC) [12][13]. These methods are used extensively on crystalline silicon wafer based solar cells and modules, but are also applied to other PV technologies, such as inorganic thin film devices. 
The method developed in this work is focused on current mapping of PV devices. This is mainly achieved by using LBIC methods, for the realisation of which a light beam scans the PV sample, with the current response being measured via the output terminals at each point of the beam scan. The diameter of the light beam spot on the sample and the movement step chosen define the resolution of the final current map. The main advantage of this technique is that the same principles that generate current in real applications of solar cells are applied in LBIC measurements. In addition, this method is universal as it can be applied to all types of PV samples, as long as they can be contacted. On the other hand, a well-known drawback of this measurement technique is that it lacks speed. The diameter of the laser spot that scans the sample is usually in the micrometre scale and scanning the entire area of the cell is necessary for a complete current map. This means that high resolution measurements require long measurement times, which also scale with increasing device area. Thus, measuring entire PV modules or full wafer cells at useful spatial resolutions is rather time-consuming, especially when compared to other spatial characterisation methods. LBIC systems can also be complicated by elaborate high-gain signal amplification requirements, in order to achieve high accuracy measurements from the weak current signal that the small laser spot induces. This means that the majority of high performance LBIC systems utilise lock-in techniques, high accuracy optical elements and precise translation stages. Such components increase the cost and complexity of these measurement systems.

In this work an alternative current mapping method is developed, which aims to mitigate the above drawbacks of LBIC systems. The method brings together current mapping of PV devices with the recently developed sampling theory of Compressed Sensing (CS) [14][15]. By applying CS theory, one can reconstruct a signal from highly incomplete or inaccurate information. Image compression techniques with loss of information are widely used in everyday life. For instance, JPEG compression is used for image files. Compression techniques such as this use transforms, such as the Discrete Cosine transform or the wavelet transform, to represent the image data in a sparse form. Only the largest basis coefficients for an accurate representation are kept. When reconstructing the signal, the non-stored coefficients are simply set to zero. This means that in most cases a large part of the information volume is simply discarded without degrading the data/image significantly.

The question that arises is whether one can acquire the compressed version of the signal more directly, by taking only a small number of measurements of the signal in the first instance. Compressed sensing provides a way of acquiring a compressed version of the original signal. This is achieved by taking only a small number of linear measurements and 
then reconstructing an almost exact approximation of this signal. More precisely, using compressed imaging, an $N$ pixel image can be reconstructed from $M<<N$ observations. Compressed imaging applications for instrumentation and measurements have started appearing in the literature, with the most established being the CS magnetic resonance imaging (MRI) for medical applications [16].

Through this work, the compressed sensing sampling method is used for the first time in PV characterisation and more generally in any PV application. In addition, it is shown that by using compressive sampling, the current mapping measurement speed of PV devices can be at least doubled, with the potential of an even further improvement. Moreover, the potential for application of CS current mapping on modules is investigated, which is something that is almost impossible with current LBIC systems. As this sampling method is conceptually different from the point-by-point scan method, novel experimental layouts needed to be developed. These measurement systems are significantly simpler than standard LBIC systems and demonstrate that CS current mapping can be realised by simple experimental layouts that are cost effective and straightforward to implement. The concept of CS current mapping is investigated in three different ways: spatially resolved simulations, an experimental setup for small samples and a projection-based measurement layout for larger samples and modules.

\subsection{Thesis structure}

Chapter 2 starts by summarising the optical and electrical properties of solar cells and presenting their operation principles. The most common spatial features that affect solar cell performance are briefly described. The most well-established spatial characterisation methods for solar cells and modules are then presented, with more extensive information about the LBIC measurement technique. In chapter 3 the methodology for CS current mapping is presented. The properties of the Digital Micromirror Device (DMD) [17], which is in all cases the core of the CS current mapping system are described. The compressed sensing method is then introduced and some of its current applications in instrumentation and measurement are presented. Finally, the application of compressive sampling to current mapping for PV characterisation is described, including some considerations of resolution and speed of this approach.

In chapter 4 spatially resolved electrical simulations of CS current mapping are realised, using the PV oriented nodal analysis toolset developed in CREST [18]. The simulations utilise 
a spatially resolved electrical model to provide an objective verification of the quality of the method, in the absence of measurement noise and experimental artefacts. Undersampling levels are investigated, as well as the impact of different features in simulated samples. Different parts of the CS process such as reconstruction algorithms and sampling matrices are evaluated, in order to be used later on during the experimental procedure.

In chapter 5 an analytical experimental implementation of a small area CS current mapping system for PV devices is presented. Different stages of the implementation are demonstrated, alongside experimental results. The CS current mapping instrument developed in this work and the results presented demonstrate the first successful implementation of a CS current mapping system reported in the literature. This measurement system based on a DMD kit, can perform not only compressive sampling but also conventional point by point sampling. This feature allows a quantitative experimental evaluation of the developed method, in comparison to the conventional raster scanning method. The features and limitation of this measurement system are presented and discussed.

In chapter 6 a projection system for up scaling the CS current mapping method is developed. A commercial projector is utilised for a proof of concept measurement system that shows that CS current mapping can be applied to large silicon wafer based cells as well as PV modules. The projector as a light source is evaluated and measurement results for PV cells and modules are presented. Some considerations for applying CS current mapping to modules are also discussed, along with the challenges and limitations when using a simple commercial projector for this application. Through this proof of concept system, the properties that a dedicated projection CS current mapping system for PV characterisation has to possess are demonstrated.

Chapter 7 is the concluding chapter, in which the findings of each chapter are summarised and brought together to build the bigger picture. Prospects for further research on this subject are suggested, highlighting the aspects of this method that can be further optimised. The contributions of this work to metrology as a whole are also discussed. A list of the resulting publications, as well as other achievements and awards related to the work are listed at the end of the thesis. 


\section{Chapter 2}

\section{Solar Cell Operation and Spatial Characterisation Techniques}

\subsection{Overview}

Photovoltaic (PV) devices are used to convert solar radiation into electricity and are mainly made of semiconducting materials. Arrays of PV cells are packed together into encapsulated modules. PV modules can then be connected in series or parallel to create larger PV arrays. In this chapter, a brief overview of the properties and operation principles of PV devices is presented. This includes a summary of specific properties of semiconducting materials that govern the performance of PV devices. Additionally, the operating principles and key parameters of PV cells are highlighted. Some of the spatially varying features that can lead to overall performance reduction are also discussed.

Following this, the most established spatial characterisation techniques are presented. Since a new spatial characterisation technique is developed and described in this thesis, it is necessary to review the existing methods that have proved their usefulness in providing spatial information of PV devices. This helps to determine the specific advantages and drawbacks of compressed sensing (CS) current mapping against well-known methods and specifies what improvements it brings to the field and specifically to laser beam induced current (LBIC) measurements. 


\subsection{Optical and electrical properties of solar cells}

\subsubsection{Semiconductors and optical absorption}

Almost all PV devices are based on specific semiconductor materials which have the property of absorbing light and transferring part of the photon energy to charge carriers (electrons). Material selection depends on solar spectrum absorption efficiency and cost of material and production processes. Crystalline silicon (c-Si) is the most common material for manufacturing PV devices, as silicon technology is well developed due to its use in the electronics industry. Moreover, silicon's absorption spectrum is well matched to the terrestrial solar spectrum. Other materials and compounds are also used for manufacturing PV devices, such as amorphous silicon (a-Si), gallium arsenide ( $\mathrm{GaAs}$ ), copper indium gallium selenide ( $\mathrm{Cu}(\mathrm{InGa}) \mathrm{Se} 2$ - $\mathrm{CIGS})$ and cadmium telluride (CdTe). However, new solar cell designs are emerging which use non-semiconductor materials, such as organic, polymer and perovskite based devices [19][20].

Most semiconductors used for PV are crystalline materials, which means their atoms or molecules are periodically aligned. This structure, coupled with the atomic properties of the component elements, is what gives them very useful electronic properties. The key characteristic of a semiconductor material for PV applications is its energy bandgap Eg. A photon that has energy higher than the energy bandgap of the material can be absorbed and excite an electron from the valence band to the conduction band, thus creating an electronhole pair as shown in Figure 2.1. The empty states left at the valence band can be regarded as positively charged carriers called holes. A simplified plot of electron energy states against crystal momentum is shown in Figure 2.2 for a direct bandgap semiconductor. The crystal momentum in this instance expresses the momentum of a phonon of the crystal lattice that contributes to the interaction. In direct bandgap semiconductors, the minimum energy of the conduction band occurs at the same value of crystal momentum as the maximum of the valence band. On the other hand, in an indirect band gap semiconductor, such as silicon, the bands are not aligned and an interaction with a phonon of the crystal structure is necessary for a photon to be absorbed or to recombine. These processes are summarised in Figure 2.2. 


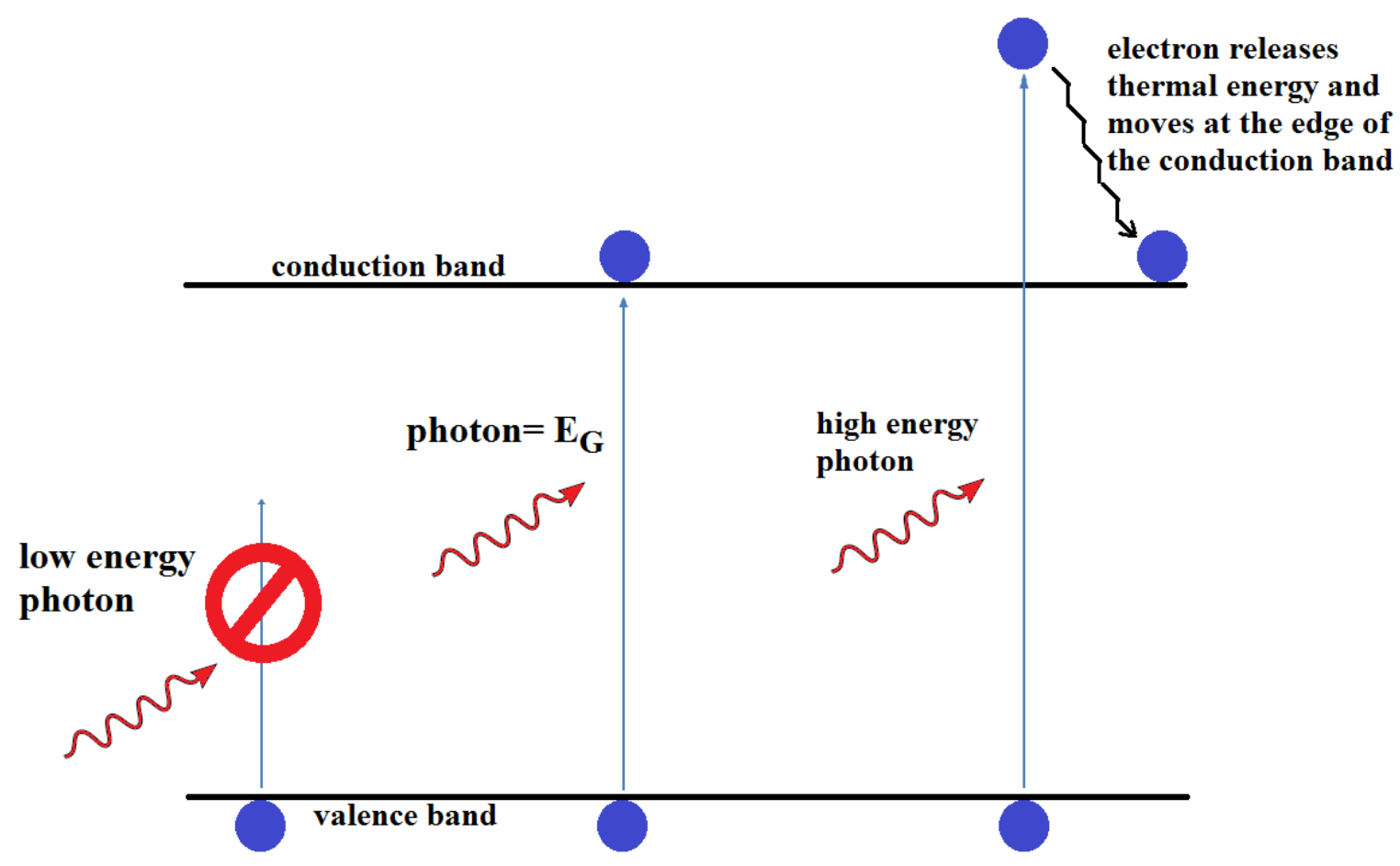

Figure 2.1 Different processes of photon absorption in semiconductors.

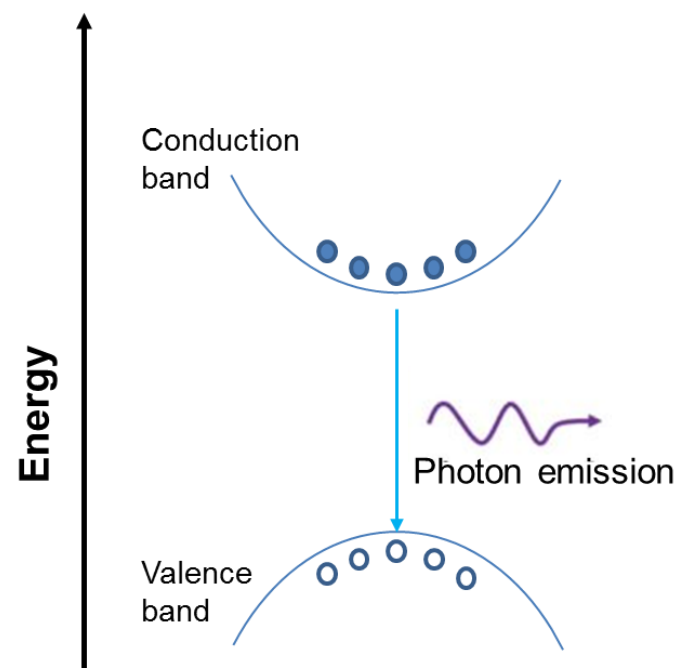

Direct bandgap

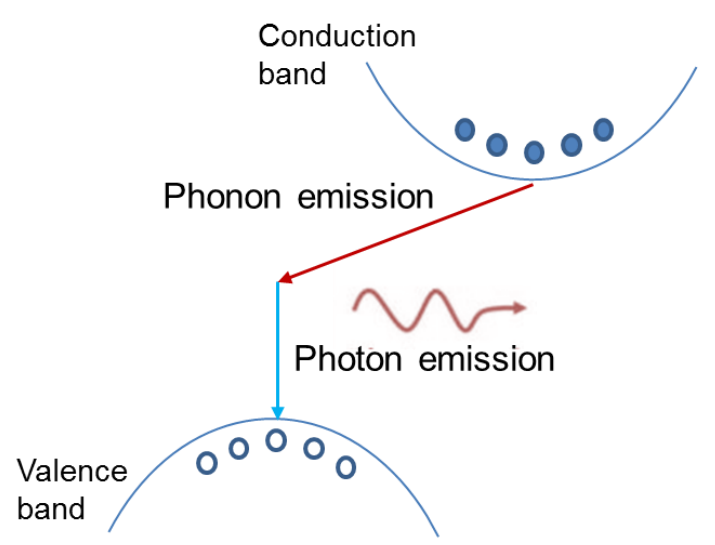

Indirect bandgap

\section{Momentum}

Figure 2.2 Photon absorption in direct and indirect bandgap semiconductors.

The number of mobile electrons and holes in a semiconductor can be controlled through the introduction of atoms of impurities, or dopants, called donors and acceptors, depending 
on whether they are inserting a negative or a positive free charge carrier. These atoms replace the atoms or molecules of the semiconductor at their lattice positions [21]. All impurities introduce additional localized electronic states into the band structure, often within the energy gap between the valence and conduction bands. Introducing donor and acceptor impurities into a semiconductor allows for the creation of n-type or $p$-type material, where electrons or holes are the primary source of electrical conduction in the semiconductor respectively. Semiconductor materials that have no introduced dopants are referred to as intrinsic semiconductors.

A significant optical parameter that determines light absorption in a material is its absorption coefficient $\alpha$. Although the optical absorption is a strong function of the thickness and geometry of a sample, the absorption coefficient is a constant for a given material and wavelength and is not affected by thickness [22]. The absorption coefficient describes the decrease in light intensity as a beam of light propagates through a material. Using the absorption coefficient, the absorption depth can also be determined. This is the inverse of $\alpha$, expressing the depth into a material where, after absorption, the remaining energy of a beam of a specific wavelength is $1 / e$ percent of its initial energy, where $e$ is Napier's constant.

\subsubsection{P-n junction and PV device operation}

A p-n junction is created at the interface between a p-type and an n-type semiconductor, as shown in Figure 2.3. Due to the high electron and hole concentrations in the $n$-type and $p$ type sides, respectively, electrons diffuse to the p-type side and holes to the n-type side [23]. This diffusion of charge carriers leaves behind exposed charges, namely: positive ions in the $\mathrm{n}$-side and negative ions in the $\mathrm{p}$-side. Thus, an electric field is built up in this region. The charge diffusion process continues until an equilibrium is created between the charge concentration and the developing electric field. This region of exposed ions is called the "depletion region" or "space charge region". A voltage bias due to the electric field is formed at the junction. Almost all photovoltaic energy conversion technologies use semiconductor materials in the form of a $p-n$ junction. When the device is illuminated, minority charge carriers will be generated. The field at the junction pulls minority carriers across the junction, which results in charge separation and reduces the probability of recombination. 


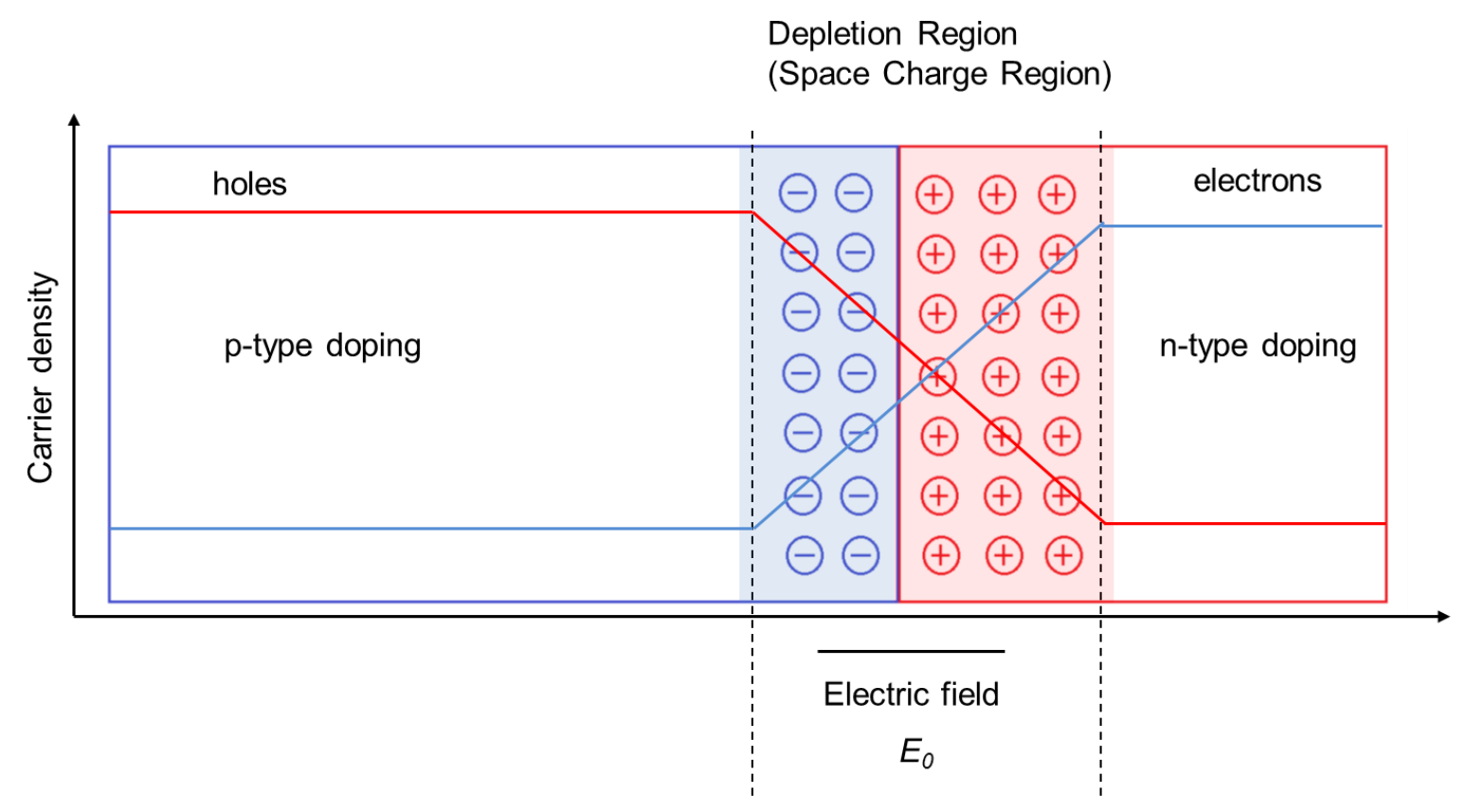

Figure 2.3 Schematic diagram of a p-n junction and charge carrier density.

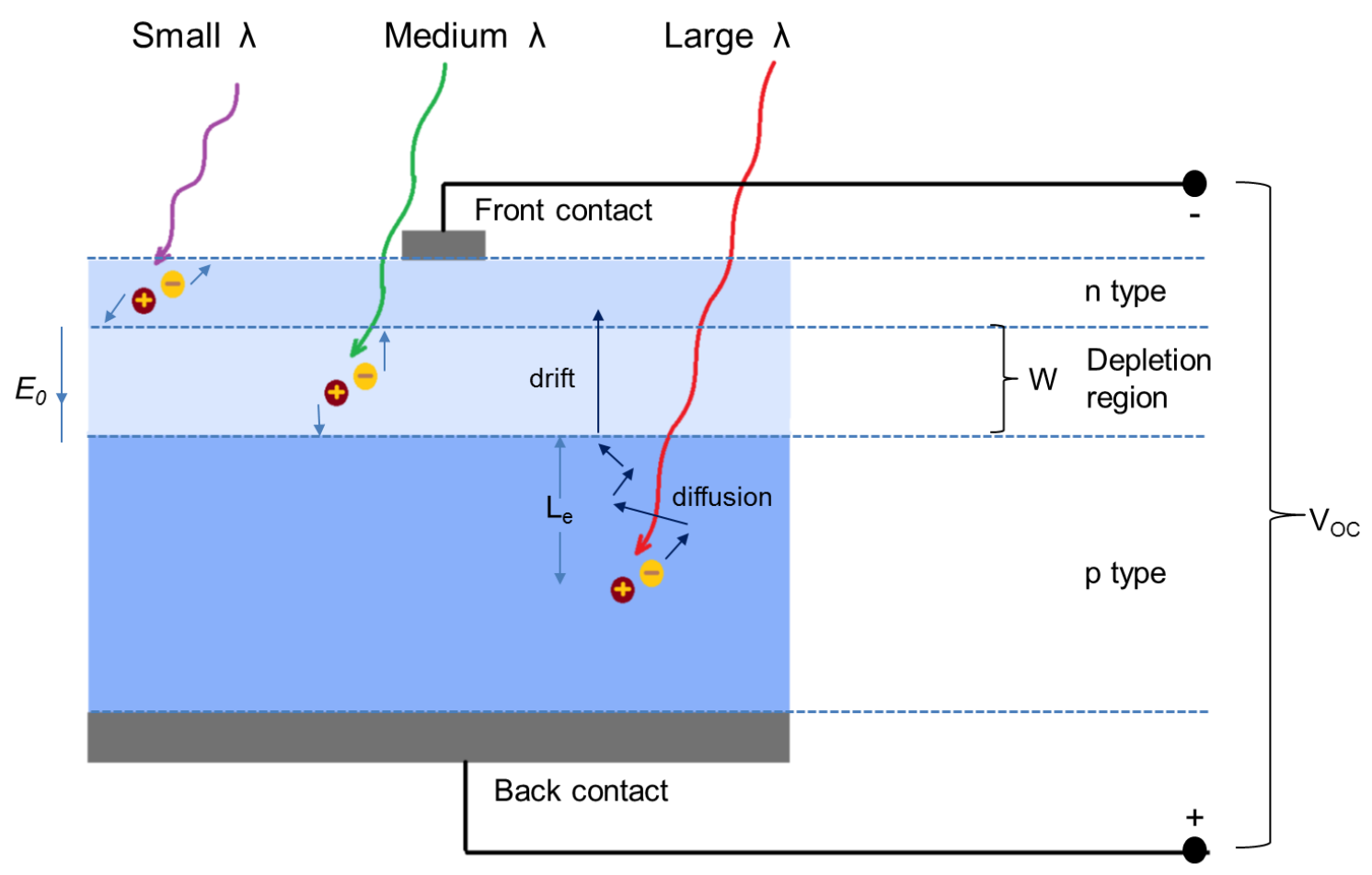

Figure 2.4 Operating principle of a photovoltaic device

In Figure 2.4, a simplified common structure of a c-Si PV device is presented. The device usually consists of a thin highly doped $n$-type region and a thick $p$-type side, while light enters 
the device from the $n$-type side. Lately, $n$-type bases are also used with thin $\mathrm{p}$-type regions to realise the junction. The depletion region has a thickness $W$ and an electric field $E_{0}$ is formed inside it [24]. Most of the incident photons are absorbed in the depletion and p-type regions, creating electron and hole pairs. In the depletion region, these pairs are instantly separated. The electron collected at the $n$-type side charges the side by -e while the hole charges the $p$ side $+e$, thus an open circuit voltage is created across the device. Longer wavelength photons are absorbed deeper in the $\mathrm{p}$-type region, where there is no electric field to drive them to the $\mathrm{n}$-type side. If their lifetime is $\tau_{e}$ then the electron will diffuse a mean distance (diffusion length) [23]:

$$
L_{e}=\sqrt{D_{e} \tau_{e}}
$$

Where $D_{e}$ is the diffusion constant of the p-type material. Electrons generated closer than $L_{e}$ from the depletion region can travel to this region through diffusion and are then driven to the $n$-type side by the electric field. Electron-hole pairs generated at a longer distance are lost due to recombination. The same principles stand for holes in the n-type region with the diffusion length of the holes being $L_{h}$.

\subsubsection{Recombination}

When excess charge carriers are generated in a semiconductor, either by light absorption or by current injection, they must recombine after the source has been switched off or disconnected [25]. Recombination in solar cells can be distinguished between bulk and surface recombination. Recombination is also classified as radiative and non-radiative. Radiative recombination dominates in pure direct bandgap semiconductors with no defects. In principle, an electron in the conduction band recombines with a hole in the valence band, emitting a photon of energy Eg, the energy of the bandgap.

Non-radiative recombination is divided between defect assisted and Auger recombination. Defect assisted or Shockley-Read-Hall recombination is described by the SRH theory [26] and does not occur in pure semiconductor materials without defects. A midgap energy state is introduced through defects in the crystal lattice or impurities. An electron can be trapped in this energy state and if a hole moves up to the same energy state before the electron is thermally re-emitted into the conduction band, then they recombine. In Auger recombination, an electron and a hole recombine, but rather than emitting the energy as heat or as a photon, the energy is given to a third carrier, an electron in the conduction band. This 
excited electron then thermalizes back down to the conduction band edge. Different recombination processes dominate at different carrier injection levels [27]. Auger recombination is important in heavily doped materials with high carrier concentrations. All the different types of recombination are schematically described in Figure 2.5.

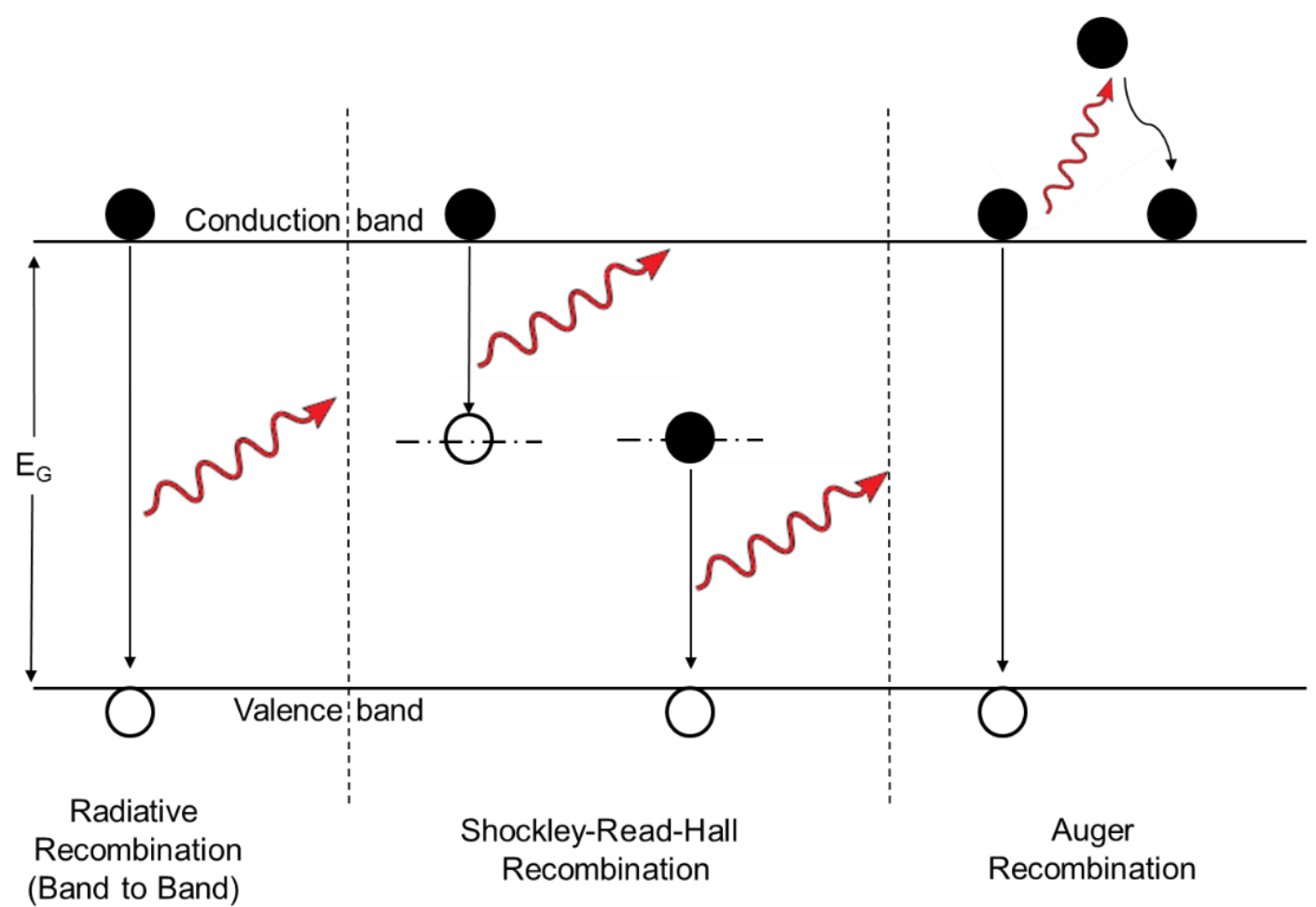

Figure 2.5. the different types of recombinations processes present in semiconductor solar cells.

Surface recombination is an important phenomenon that affects the dark saturation current and quantum efficiency of solar cells. Since the surface of the solar cell is a disruption of the crystal lattice, it is a site of particularly high recombination. Surfaces and interfaces introduce midgap states that can be described as broken (or strained) bonds and impurities, in other words, recombination sites. To reduce surface recombination in solar cells, a layer that introduces a back surface field is applied that prevents minority carriers from reaching the surface and hence, reduces recombination. For surface passivation, an oxide layer is usually used. In these cases, surface recombination velocity is strongly dependent on deposition conditions.

The carrier lifetime of a material is defined as the average time that a minority charge carrier lives until it recombines. The usual value of carrier lifetime for high quality monocrystalline silicon is approximately $1 \mathrm{~ms}$. Equivalently, the carrier diffusion length can also be defined as the average distance a carrier can travel from the location of generation until it 
recombines. For silicon, this can be some hundreds of micrometres. These two parameters are indicators of material quality and suitability for solar cell use.

\subsection{Solar cell operation principles}

For electrical modelling purposes, a solar cell is usually represented by the equivalent circuit displayed in Figure 2.6, known as the one diode model. This includes a current source $I_{\mathrm{ph}}$, a parallel diode with current $I_{\mathrm{D}}$, a parallel resistance $R_{\mathrm{sh}}$ that represents leakage current across the junction and a series resistance $R_{\mathrm{s}}$. The I-V characteristic is described by equation [28]:

$$
I=I_{p h}-I_{o}\left[e^{\frac{V+I R_{s}}{n V_{T}}}-1\right]-\frac{V+I R_{S}}{R_{s h}}
$$

Where $n$ is the diode ideality factor (between 1 and 2 for a single junction cell), $I_{0}$ is the saturation current, and $V_{\mathrm{T}}$ is the thermal voltage given by $V_{T}=\frac{k T}{q}, k$ being Boltzmann's constant, $T$ is the temperature of the cell and $q$ the elementary charge.

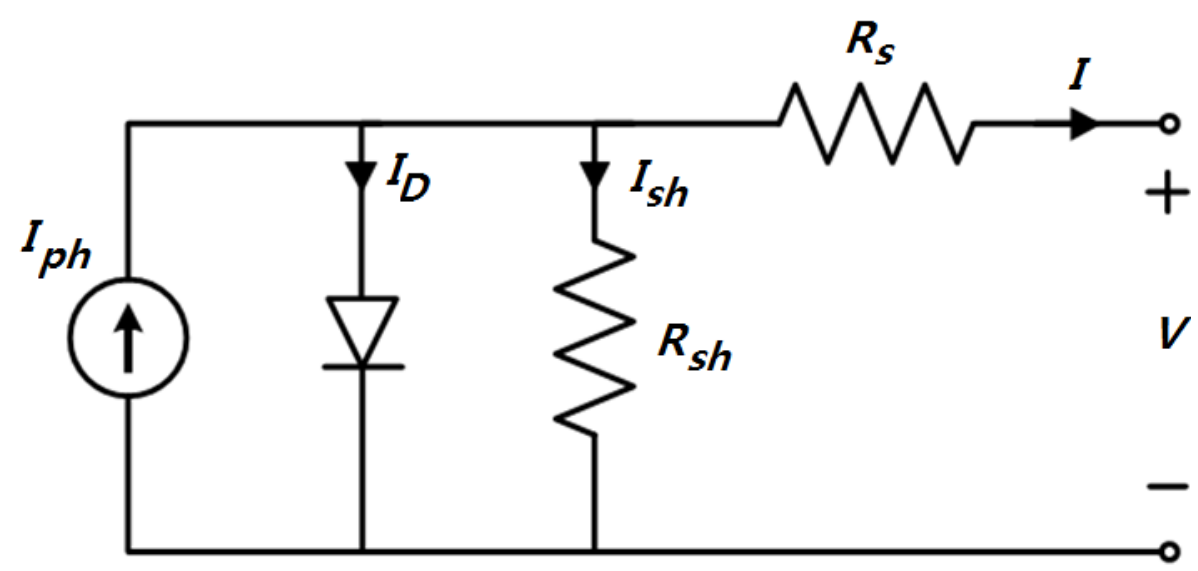

Figure 2.6. The one diode model for PV cells

The cell generates maximum power $P_{\mathrm{M}}$ at a voltage $V_{\mathrm{M}}$ and current $I_{\mathrm{M}}$, and it is convenient to define the fill factor (FF) as: 


$$
F F=\frac{I_{\mathrm{M}} V_{\mathrm{M}}}{I_{\mathrm{SC}} V_{\mathrm{OC}}}=\frac{P_{\mathrm{M}}}{I_{\mathrm{SC}} V_{\mathrm{OC}}}
$$

Where $I_{S C}$ and $V_{O C}$ are the short circuit current and open circuit voltage of the cell, respectively. These parameters are labelled in the typical I-V characteristic curve shown in Figure 2.7.

The maximum efficiency is the ratio between the maximum power and the incident light power, given by the equation:

$$
n_{\text {max }}=\frac{P_{M}}{P_{\text {incident }}}=\frac{I_{M} V_{M}}{A G}
$$

Where $A$ is the cell area $\left(\mathrm{m}^{2}\right)$ and $G$ is the incident irradiance $\left(\mathrm{W} / \mathrm{m}^{2}\right)$.

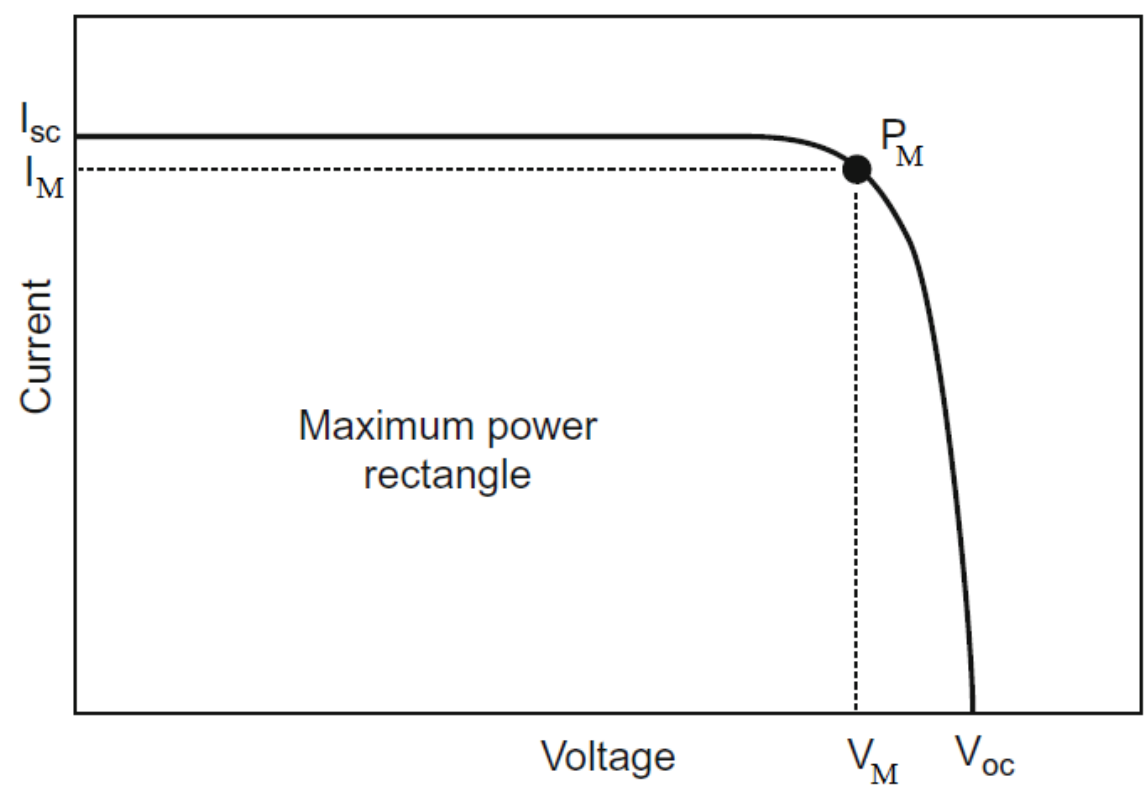

Figure 2.7 The I-V curve of an ideal solar cell.

In practice, the I-V curve of a real solar cell is different from the above case. For a more physically meaningful modelling, a two-diode model is often used to fit an observed curve, with a second diode [29]:

$$
I=I_{p h}-I_{01}\left[e^{\frac{V+I R_{S}}{V_{T}}}-1\right]-I_{02}\left[e^{\frac{V+I R_{S}}{2 V_{T}}}-1\right]-\frac{V+I R_{S}}{R_{s h}}
$$


The second diode $I_{02}$ in parallel with the first represents recombination in the junction and its ideality factor is set to 2 . The diode $I_{01}$ represents recombination in the bulk of the device and its ideality factor is set to 1 .

Another significant metric of solar cell performance is the quantum efficiency. This is the ratio of the number of carriers collected by the solar cell to the number of photons of a given energy (wavelength) incident on the solar cell. One can define external (EQE) and internal quantum efficiencies (IQE) where all photons incident on the cell surface are considered in the value of the EQE, but only photons that are not reflected out of the PV device are considered in the value of IQE. The values of the internal and external quantum efficiency are measured using interference filters or monochromators, in order to access the performance of a PV device as a function of the wavelength of the incident light. Quantum efficiency can be expressed in terms of spectral response:

$$
\begin{gathered}
E Q E(\lambda)=S R_{\text {ext }}(\lambda) \cdot \frac{h c}{q \lambda} \\
I Q E(\lambda)=\frac{E Q E(\lambda)}{1-R(\lambda)} \\
S R_{\text {ext }}(\lambda)=\frac{I_{S C}}{G(\lambda) A}
\end{gathered}
$$

Where $S R_{\text {ext }}$ is the external spectral response of the solar cell under consideration, $\lambda$ is the wavelength of the incident light, $h$ Planck's constant, $q$ the electron charge, $c$ the speed of light in vacuum, and $G$ the intensity of the incident light and $A$ the area of the sample. 


\subsection{Spatial features affecting the performance of PV devices}

Non-uniformity of material properties and local defects have an impact on overall performance of solar cells and eventually modules and systems. Local features and defects present in silicon wafer based solar cells and modules are usually different from defects and structures detected in thin film devices. This is mainly because crystalline PV modules and thin film PV modules possess a very different design, different properties and are produced using different manufacturing processes. In addition, new emerging technologies introduce new types of defects, spatial properties and degradation profiles that make spatial characterisation crucial for solar cell material development. While there is a huge variety of defect types and local features in PV devices that can affect performance, in this section the most common ones will be summarised. These features concern mainly PV device material properties and features at cell level, therefore they do not include additional effects at module scale, such as delamination, discoloration or module breakage.

\subsubsection{Cracks}

Cracks in PV devices are a very common occurrence, mainly in crystalline wafer solar cells and modules. It is difficult to avoid cracks in PV modules and even harder to evaluate quantitatively their influence on module lifetime and durability. Cracks and micro cracks usually have only a minor effect on the power of a PV module if there are no areas of cells separated and electrically disconnected. For new modules, the reduction of power due to cracks is usually lower than $2.5 \%$ [30]. However, as a PV module containing cracks ages, it is exposed to severe thermal and mechanical stresses. This potentially results in crack propagation that could electrically disconnect large areas of PV cells and reduce the power at the module terminals. In addition, even if only one cell suffers from significant power losses due to cracks, this cell affects the performance of the whole sub-string of cells within the module.

It has been shown that the initial orientation of cracks in cells plays a significant role in crack propagation and subsequently power losses after thermal and mechanical stresses. Cracks parallel to the busbars are more prone to cause electrical separation of cell areas while cracks perpendicular to the busbars seem to have a much smaller impact [31][32]. Production processes such as soldering can also cause cracks [33]. Transport, handling and installation cause additional mechanical stresses, while cell type and thickness also have an impact on crack propagation due to these mechanical stresses [34][35]. On the other hand, if a crack is 
already present in the wafer before the emitter deposition, it can lead to a strong ohmic shunt. This is because an emitter layer may be established across the crack and short the emitter against the base contact, creating a linear (ohmic) shunt along the crack [36]. An electroluminescence image of a commercial mc-Si PV module is presented in Figure 2.8. A large number of cracks and several disconnected areas that reduce the performance of the module are visible in the image. Such defects are very typical for such PV modules.

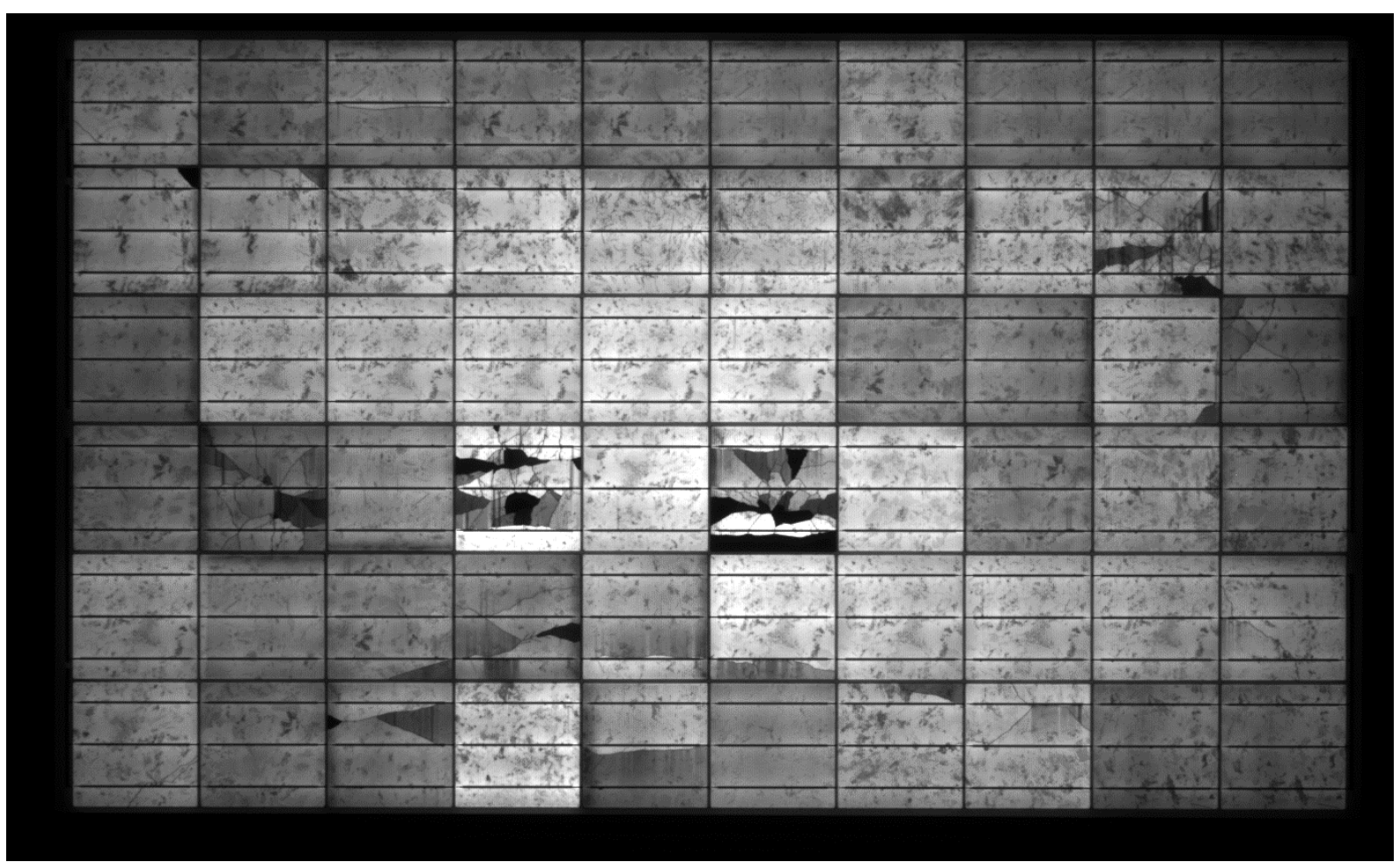

Figure 2.8. Electroluminescence image of a commercial PV module. Cracked cells with disconnected areas are visible.

\subsubsection{Shunts}

As described in [36] a shunt can be defined as "any position in a solar cell showing under forward or reverse-bias a dark-current contribution additional to the diffusion current". In other words, local dark leakage current sites can be characterised as shunts. In the cases that such shunts are sufficiently strong, the fill factor and subsequently the efficiency of a device can be reduced, as presented in Figure 2.9. Moreover, shunts can lead to high current flow in these defective regions, which may result in the creation of hot spots. This effect becomes critical in the case of an illuminated PV module; when the cell that contains a strong shunt is 
shaded or electrically mismatched, a large current generated by the illuminated cells is forced to flow through its shunted region, creating a strong reverse bias. The result can be high local overheating that can permanently damage the PV cell and subsequently the module [37][38].

Wafer based crystalline silicon solar cells almost always contain shunts that result in some local leakage current. The most common are edge shunts, where the emitter may not be perfectly separated from the rear contact of the device [39]. Other shunts present in PV cells may be due to scratches on the emitter or cracks formed in the wafer before the emitter diffusion [36]. Shunts caused by damage to the emitter of the device most often show the electrical behaviour of a Schottky contact.

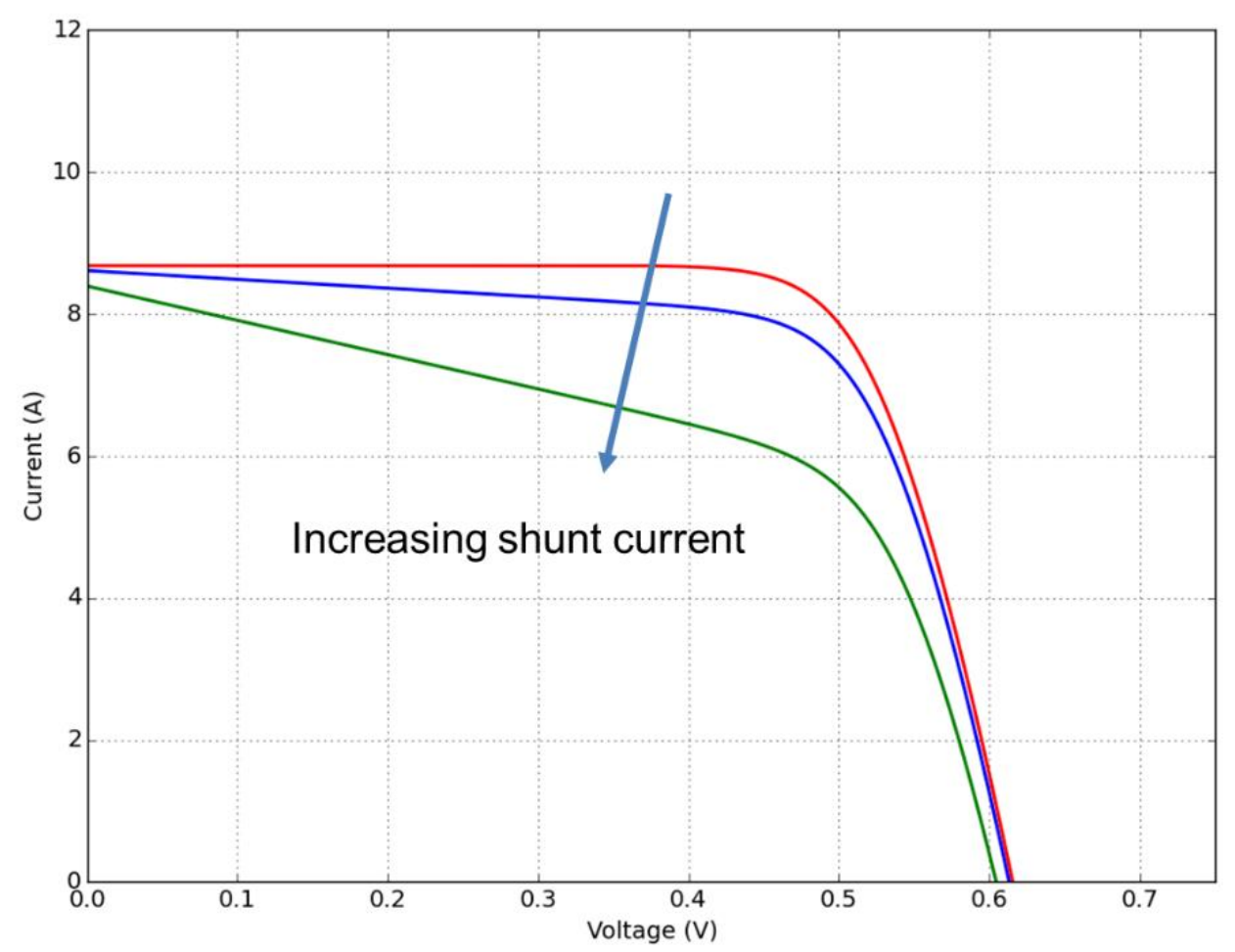

Figure 2.9. The effect of increasing shunt current from increasing shunt density on PV device performance

Shunts are not only an issue in wafer based silicon solar cells; they also appear in thin film devices. Shunts in CIGS PV modules can be observed using electroluminescence imaging, although they have a minor effect on device performance [40]. Shunts also appear in organic PV devices [41]. It has been shown that the leakage current due to shunts exhibits very similar features in all thin film PV devices [42]. This is mostly because the formation of the usual shunting defects in such devices is very similar; non-uniform deposition or fabrication can 
lead to the metals or transparent conductive oxides coming into direct contact with the absorber layer, to form a parasitic metal-semiconductor structure.

\subsubsection{Material micro-structure}

The microstructure properties of the active material are essential for any multi crystalline material used for PV applications. Material structure properties of PV devices can lead to local features that affect overall device performance. Multicrystalline Si PV cells are not only affected by defects induced during or after the manufacturing process, but they are also influenced by the inherent structural properties of the initial silicon material. The most common are grain boundaries and dislocations, which are always present and whose impact depends on material quality and the technique of initial material production [43].

Grain size mainly depends on the manufacturing technique used to produce the silicon wafers. Grain boundaries, if electrically charged, can attract or repel minority charge carriers and consequently can become active recombination centres for photo-generated charge carriers. The electrical activity of grain boundaries is determined by their impurity decoration (specifically by transition metals) and strongly increases with higher impurity concentrations. Nevertheless, it has been demonstrated that at high contamination levels, larger precipitation of metal impurities in grain boundaries is usually preferable to having a higher density of smaller precipitates and point defects distributed in the bulk of the material [44]. Configuring growth conditions, metal contaminants can be controlled and their impact on device performance can be minimised.

Dislocations in crystalline silicon are formed during crystal growth, as plastic deformation take place in order to reduce the thermal stresses. Some of these dislocation networks can be strong recombination centres and consequently they can have a negative impact on the lifetime of minority charge carriers, which dominates the PV device performance. In similar fashion to grain boundaries, dislocations are sites of high metal impurity concentrations [45], which determine their electrical activity.

Dislocations and grain boundaries are also present in the active material of CdTe and CIGS solar cells. In the case of CdTe devices, although the effect of grain boundaries on untreated CdTe has been recognised as a limiting factor to performance, the role of grain boundaries in finished (treated) devices is still controversial [46]. While using specific experimental techniques it has been revealed that grain boundaries in treated devices can significantly enhance collection current [47], other studies show that grain boundaries have 
a negative effect on performance [48]. More research in this matter using additional characterisation techniques is necessary in this case. Grain boundaries also influence the performance of CIGS solar cells [49][50]. It has been demonstrated recently that chemical modification of grain boundaries in CdTe and CIGS solar cells is possible, in order to tune and suppress their negative effects on solar cell performance [51].

\subsubsection{Effects of degradation}

Material degradation in all types of PV devices is a major source of non-uniformities and local defects. Regarding crystalline silicon PV devices, alongside module impacts such as corrosion, delamination and discoloration, degradation can also increase crack and shunt density in PV cells of a module [52]. It has been demonstrated that ageing of PV cells and modules usually leads to increasing crack density and broken front contact fingers [53][54]. This has a major effect on power losses, as series resistance increases while the fill factor decreases. Most material degradation effects in crystalline Si based solar cells such as cracks, shunts and broken fingers are localised features that eventually affect the overall performance of the device. Degradation issues also affect inorganic thin film solar cells. Aside from module issues, ageing CdTe devices can also result in a decrease in local contact quality, creating localised areas of underperformance [55].

Degradation and stability behaviour always leads to local defects and non-uniformities and is extremely important for new emerging materials for PV devices, such as organic and perovskite PV devices [56]. The most frequent local defects introduced in such material types are degraded areas due to decomposition of the active material [57]. The characterisation of such spatially varying features is extremely important for the progress and development of such novel concepts for solar cell materials. 


\subsection{Spatial characterisation of solar cells}

Characterization has a significant role in the advancement and realisation of photovoltaic (PV) technologies. More specifically, spatial characterization of the optical, electrical and material properties of PV devices is used for the detection of various kinds of defects, which can arise due to production processes, material properties or degradation of the device under study. Thus, PV device characterization leads to an increase of PV product quality through detection and prevention of production induced defects and better understanding of material and degradation properties of PV devices. Moreover, characterization techniques are also necessary for research into new PV materials and designs and for optimization of manufacturing methods.

The most common methods for spatially resolved non-destructive measurements are Light (or Laser) Beam Induced Current Measurements (LBIC), luminescence methods such as Electroluminescence (EL) and Photoluminescence (PL) and finally Lock-in Thermography (LIT). Since in this work a new method is developed with more similarities to LBIC measurements, this specific technique is described in more detail here. Luminescence and thermography methods will also be reviewed in this chapter, to provide a means of comparison with LBIC and the CS current mapping method under development.

Luminescence techniques such as Electroluminescence (EL) and Photoluminescence (PL) are much faster as they can capture images in seconds [58], but cannot deliver such a high resolution as the LBIC technique. With such methods, qualitative analysis can be realised for imaging specific parameters of a PV sample, such as series-resistance, implied voltage or saturation current density imaging as will be discussed later. Lock-in Thermography (LIT) is also faster than LBIC, though slower than EL or PL, but usually yields lower resolution images [59]. Using LIT imaging, spatial information about several solar cell parameters can be acquired. The main disadvantage of the aforementioned methods for acquiring a sample's performance parameters is that they rely on a number of assumptions in order to be applied. One of the assumptions is that the short circuit current is spatially homogeneous. This has been shown to be untrue in most cases and large local variations of the short circuit current density are observed in the majority of PV samples [13].

A more significant argument is that electronic properties of solar cells, such as the diffusion length of minority charge carriers, are usually extracted from quantum efficiency (QE) data. The values of these properties are typically measured in an energy range slightly above the band-gap energy, where the QE is still close to its maximum [60]. For this reason, quantitative current mapping provided by LBIC measurements is essential for directly 
assessing the electronic, optical and material properties of PV devices. Particularly for research devices, new materials and new design concepts where any assumptions are difficult to rely on, LBIC systems can provide accurate local quantification of the electrical properties of a sample. For these reasons, LBIC measurements are often used as reference for validation of luminescence methods.

LBIC measurements can be applied to solar cells and modules (encapsulated or not), but not to wafers or, more generally, samples with no way of contacting. Through this section, it will become clear that the different characterisation methods of PV devices are not competing with each other but rather are complementary. Different characterisation methods are necessary for different cases, samples, parameters to be measured, time margins or for validation of results [61][62]. Apart from a brief description of each characterisation technique, the LBIC systems in CREST will be described in this section.

\subsubsection{Light beam induced current (LBIC) measurements}

\subsubsection{Overview}

Light/Laser Beam Induced Current (LBIC) imaging is a non-destructive characterization technique, which can be used for mapping the current response of PV cells and modules. For its realisation, a light beam scans the PV sample and the induced current is measured for each point. Using a light beam to probe a $p-n$ junction was introduced as a method for the first time in the 50's by Goucher et al [63], for scanning a Germanium p-n junction with infrared light. Since then, such methods have been widely used for characterizing semiconductors. The application of LBIC to solar cells was introduced in the late 70's and early 80's [64][65][66]. A variety of different system approaches have been proposed, allowing LBIC measurement systems to deliver spatial maps of electrical properties, reflectivity, performance parameters and material properties of solar cells. However, this technique lacks speed as the small spot size (usually of a diameter of some micrometres) has to scan the entire area of the cell for a complete cell map, which means the smaller the spot size the lengthier the measurements. On the other hand, the resolution that can be achieved with LBIC, which can be as good as several micrometres, cannot be accomplished with other imaging methods.

One of the first analytical descriptions of an LBIC measurement system is found in [66]. The setup consisted of two He-Ne lasers, one of which operated at $632.8 \mathrm{~nm}$ and the other at $1150 \mathrm{~nm}$. One of the lasers was selected for the scan, with beam being focused on the sample using a lens. The cell was placed on an $x-y$ translation stage with a motor drive for making line 
scans of the sample. Although modern systems differ somewhat from this setup, they all share some common principles. These are: a light source; an optical system for manipulating and focusing the beam on the sample; a system that enables movement of the sample in relation to the beam; and the necessary electronic equipment and software for recording the signal and subsequently realising the LBIC measurements.

\subsubsection{Experimental approaches and development}

The first and most significant part of an LBIC system is the light source. The most common approach is to use one or more laser sources in the wavelength range of $400 \mathrm{~nm}$ to $1150 \mathrm{~nm}$. For scanning crystalline silicon solar cells, the wavelength range most widely used is from $600 \mathrm{~nm}$ to $1000 \mathrm{~nm}$, as shorter wavelengths cannot penetrate more than $1 \mu \mathrm{m}$ into silicon. In addition, for longer wavelengths there is parasitic absorption from free charge carriers and the back surface of the solar cell [67]. Usually multi-wavelength systems are used for providing more information about the properties of the sample under consideration. The initial aim of LBIC systems that used multiple laser sources was to obtain an approximation of the local effective diffusion length of silicon devices, through LBIC measurements [68][69]. This was achieved by using lasers of several wavelengths from $750 \mathrm{~nm}$ to $900 \mathrm{~nm}$ and applying P. Basore's IQE analysis [70][71]. Multi wavelength LBIC systems allow measurement of a larger variety of samples and not only crystalline silicon. Local spectral response at the available wavelengths can also be measured when more wavelengths in the solar cell's operating range are available [13]. Different depths or layers of a PV device can be also accessed and compared when using multiple wavelengths [72]. Concentrated solar light has been used as a light source in an LBIC system [73]. Recently a local solar simulator which is simultaneously an LBIC system was reported [74]. This system used a super continuum laser along with optical elements to create a beam with a spectrum that simulates the standard solar spectrum.

It has been shown than semiconductor lifetime and subsequently the diffusion length of minority carriers depends on the carrier injection level, which is the level of light intensity incident on the cell or the level of forward bias [75][27]. For this reason, bias illumination was introduced to LBIC systems in several cases at a very early stage, especially when the intensity of the light used for scanning the cell was low [76]. The bias illumination is either inserted in the optical system [76] or applied from the side onto the sample [77]. Uniformity of bias light is important for accurate measurements, especially in nonlinear samples or when mapping 
multiple cell devices, as indicated in [78]. Modern systems that include bias light utilise arrays of lamps to achieve uniform bias light of adequate intensity [12][79].

Control of the laser or light beam and focusing it to a small spot on the sample is very important for LBIC systems. Early systems used an objective lens just above the sample for focusing the beam to a small spot, while the sample was placed on a $x-y$ translation stage [80][66]. This approach is also the most widespread in modern experimental setups as it is convenient for scanning relatively small samples. However, different approaches are used for module-scale devices and generally larger samples. The CELLO (solar CELL LOcal characterization) LBIC system [12] utilizes a piezo-controlled mirror system for guiding a beam of $100 \mu \mathrm{m}$ spot size focused on the sample, which is in a stable position. This approach was introduced very early in LBIC systems [81]. A similar approach is presented in [79] where the beam is guided to the required position on a solar cell or module by a pair of mirrors. Using a mirror system is convenient for larger samples, when movement with an $\mathrm{x}$-y translation stage is not a practical option. Background illumination is also more easily integrated into these systems, as the position of the optical system away from the sample prevents shadowing. On the other hand, systems that use mirrors can introduce interference patterns in current maps when scanning PV devices that are encapsulated in glass, due to multiple internal reflections of the beam between the sample surface and the outer glass surface [81][78]. Reflectivity measurements are also difficult to implement in such systems

In an LBIC setup, the beam that scans the solar cell is focused to a very small spot on the cell, with a size of several to some hundreds of micrometres, usually with the utilization of a microscope objective. Even initial systems managed to achieve a spot of less than $10 \mu \mathrm{m}$, utilizing microscope objectives, pinholes and laser sources [80][82]. Using an optical system of several lenses and a pair of mirrors can provide an adjustable spot size, with a range of $2 \mu \mathrm{m}$ to $100 \mu \mathrm{m}$, as reported in [83]. For achieving such a small spot size, a well collimated light beam with a small diameter is required. It is possible to achieve sub-micron resolution levels in LBIC measurements, by adopting a confocal microscope configuration, as it is reported in [84]. If optical fibres have to be used, single mode fibres are preferable because of their small core size and small numerical aperture [85][72]. When a focused beam with spot sizes of the order of $1 \mu \mathrm{m}$ is used, the local laser intensity can easily become large enough to cause local heating effects. As a consequence, extra care has to be taken for reducing the light power density while reducing the spot size in order to avoid such effects.

Over time, more features have been added to the initial elementary setup for measuring more electrical, optical and material properties of samples. As presented in [80], the LBIC 
measurement setup utilizes a beamsplitter for monitoring the incident beam intensity. This approach has been adopted for almost every system that has been presented in the literature, as measuring the intensity of the beam while scanning a sample enables both monitoring the stability of the light source and being able to calculate local EQE of the scanned solar cell for the specific wavelength. By monitoring the incident light intensity, even small intensity fluctuations can be cancelled out. This is necessary even with the most stable light sources. It is especially necessary for outputs of optical fibres, through which light is sometimes guided from the light source to the optical system [85]. Any minor movement of the optical fibre will slightly alter the output intensity of the fibre. Measuring the reflected beam from the sample is also important as reflectivity maps can be produced [86]. Moreover, the reflectivity is necessary when calculating IQE, in order to acquire approximations for the diffusion length. For efficient measurement of the local $I_{s c}$ of a sample, lock-in amplifiers synchronized to the laser beam modulation signal were introduced into LBIC setups at a very early stage [87].

The latest improvements in LBIC system have moved the spot size to a sub-micron level, using microscopy configurations, for thorough investigation of localized defects, especially in thin film PV devices [88][89][90]. Advancements in laser technology have also benefitted LBIC system development, as supercontinuum lasers have been used recently [74][91]. Supercontinuum lasers have a very broad spectrum, covering the whole useful range for PV devices and delivering high power of more than 20W. Due to the broad range of emitted spectrum, parabolic mirrors are used for focusing. On the contrary, there has been little improvement of the speed of the LBIC technique, which means scans are still time consuming, especially when compared to other imaging methods. A significant time improvement was achieved by introducing an on-the-fly mode for LBIC systems [92][93]. When an LBIC system works in this mode, the laser spot moves continuously on the sample while measurements are acquired, without the spot stopping at each point. Measurements are significantly accelerated, although some minor distortions are inserted in the current maps. In the same work it was also demonstrated that specific areas of the sample can be selected and measured with different levels of resolution, achieving a resolution of up to $1 \mu \mathrm{m}$.

LBIC measurements are also useful in research of alternative architectures of crystalline silicon solar cells. Current mapping of Interdigitated Back Contact (IBC) solar cells revealed that although IBC cells avoid optical shading losses by placing the metal grids on the backside of the cell, electrical shading losses still exist, due to rear side recombination in the regions of the base fingers [94][95]. Diffusion length measurements using an SR-LBIC setup have helped the optimization of metal wrap through (MWT) solar cells, by detecting areas of lower 
diffusion length [96]. Recently, a very high resolution LBIC setup with a scanning spot size of less than $5 \mu \mathrm{m}$ was utilized to investigate local defects in the a-Si/c-Si interface of silicon heterojunction with intrinsic thin layer (HIT) cells [97].

The LBIC measurement technique is a useful characterization tool in research of novel thin film devices, as its application has been demonstrated not only on new inorganic material devices but also on organic and dye-sensitized solar cells (DSSC). Current mapping and performance parameter characterization of an InGaP/InGaAs/Ge triple junction solar cell using an LBIC system is reported in [98] while the local EQE of a CZTSe solar cell is delivered in [99]. Spatial current mapping and degradation studies have been performed for organic solar cells [100] and modules [101]. LBIC maps in organic devices provide valuable insight into spatial distribution of photocurrent and its subsequent degradation behaviour, by determining whether the loss is occurring uniformly over the sample or locally as in the case of pinhole formation [102]. The use of LBIC scans on DSSC devices is limited, as the very slow response time of DSSCs in the decay process makes this method extremely time consuming [103]. However, some new methods have been explored for the utilization of LBIC measurements on such devices [104][105]. Silicon nanowire-based solar cells on glass have also been characterized using the LBIC technique, as presented in [106].

\subsubsection{The LBIC systems in CREST}

Two LBIC systems are available at CREST, each using different principles and serving different purposes. The initial large area system able to perform measurements on both PV cells and modules is presented in Figure 2.10. The system includes three different laser sources, which have wavelengths of 410,633 and $785 \mathrm{~nm}$. A piezo electric mirror system is utilised to realise the scans and background bias light is applied with an array of lamps. Measurements take place in a large dark chamber. The lasers are frequency modulated and a lock in system is used for accurate measurements. The spot size of the beam on the sample is approximately $150 \mu \mathrm{m}$. The system has been analytically described in [79][78]. 


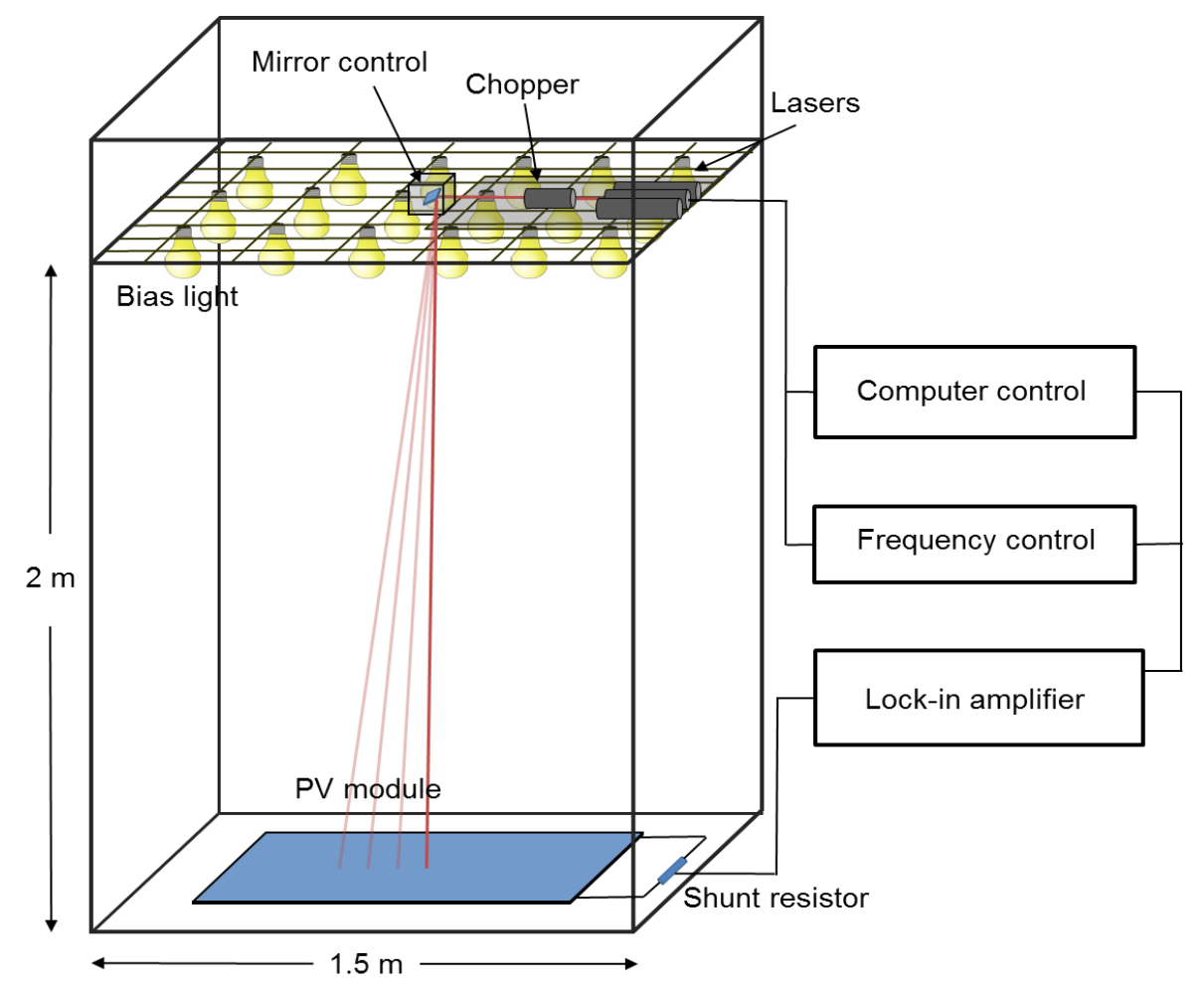

Figure 2.10. The initial large area LBIC system in CREST, for solar cells and modules.

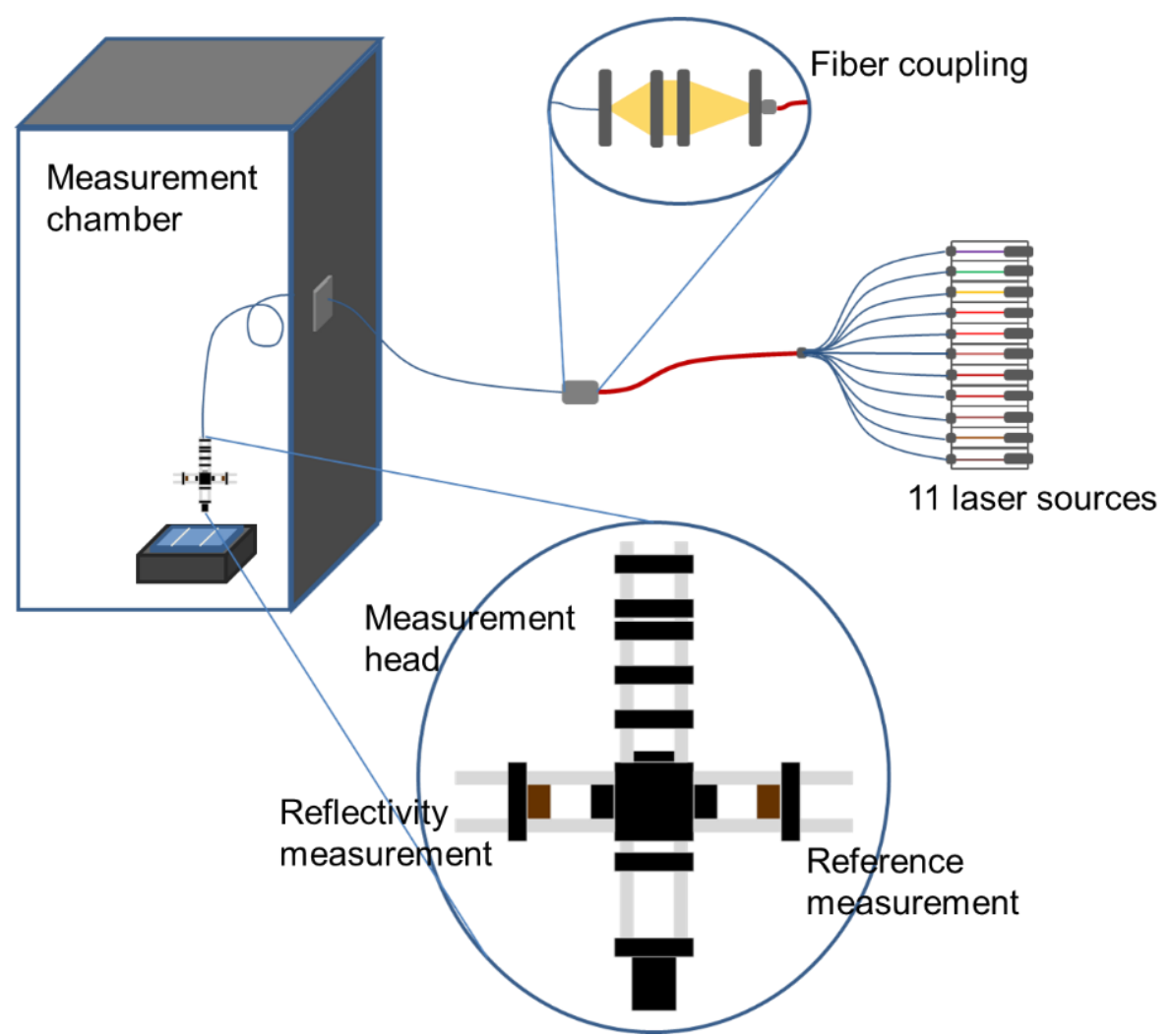

Figure 2.11 the small area LBIC system in CREST, consisting of 11 laser sources. 
The second and more recent laser system is a small area LBIC measurement setup [107]. It consists of 11 laser sources, frequency modulated, fiber coupled into one laser beam. A multimode fiber guides the light to the measurement head. The output goes through a beamsplitter; a part of the beam propagates towards the sample and another part towards a photodiode for reference measurement of light intensity. A microscope objective lens focuses the beam to a small spot on the sample, while collecting the reflected light, which is guided to the reflectivity measurement photodiode. A lock-in amplifier is used for measuring the response for each of the modulated laser sources. In this way, measurements with all 11 laser sources can be performed simultaneously. Part of this thesis work included the design of the optical configuration of this small area LBIC system.

\subsubsection{Electroluminescence imaging}

In electroluminescence (EL) imaging, a PV device is electrically contacted and forward biased, resulting in electrons being excited into the conduction band of the semiconductor device. During this forward bias, a CCD array or similar camera detector captures an image of the emission due to radiative recombination of minority carriers. The technique was introduced by Fuyuki et al. [9] as a contactless photographic surveying method for the analysis of silicon solar cells and photovoltaic modules. The typical set-up for an electroluminescencebased inspection system comprises the solar cell sample connected to a power supply for forward biasing and a silicon charge-coupled device (CCD) camera used to capture the picture, which is then processed for correction of imaging artefacts [108]. A schematic of the system layout of the small area EL system at CREST is presented in Figure 2.12 [109], along with an EL image of a silicon solar cell.

It has been demonstrated that EL imaging is a very useful technique for fast spatial imaging of PV cells and modules. Various faults regarding PV module damage are detectable by EL imaging, such as cracks, disruptions within the metal front contact grid of the solar cell, shunts between base and emitter, and disruptions of the electrical interconnections [110]. The main disadvantages of EL imaging are that images are dominated by radiative recombination and series resistance losses and the images do not accurately reflect the performance of the device under actual operating conditions. The current paths when forward biasing a PV device are different to those when illuminating it [111]. When injecting 
current into a PV device, the current density is larger in the cell areas close to the top metal fingers and lower in the central region of the device, away from the metal grid. On the other hand, under actual operating conditions of illumination, the current flow in the cell is reversed, resulting in a higher operating voltage in the central emitter region.
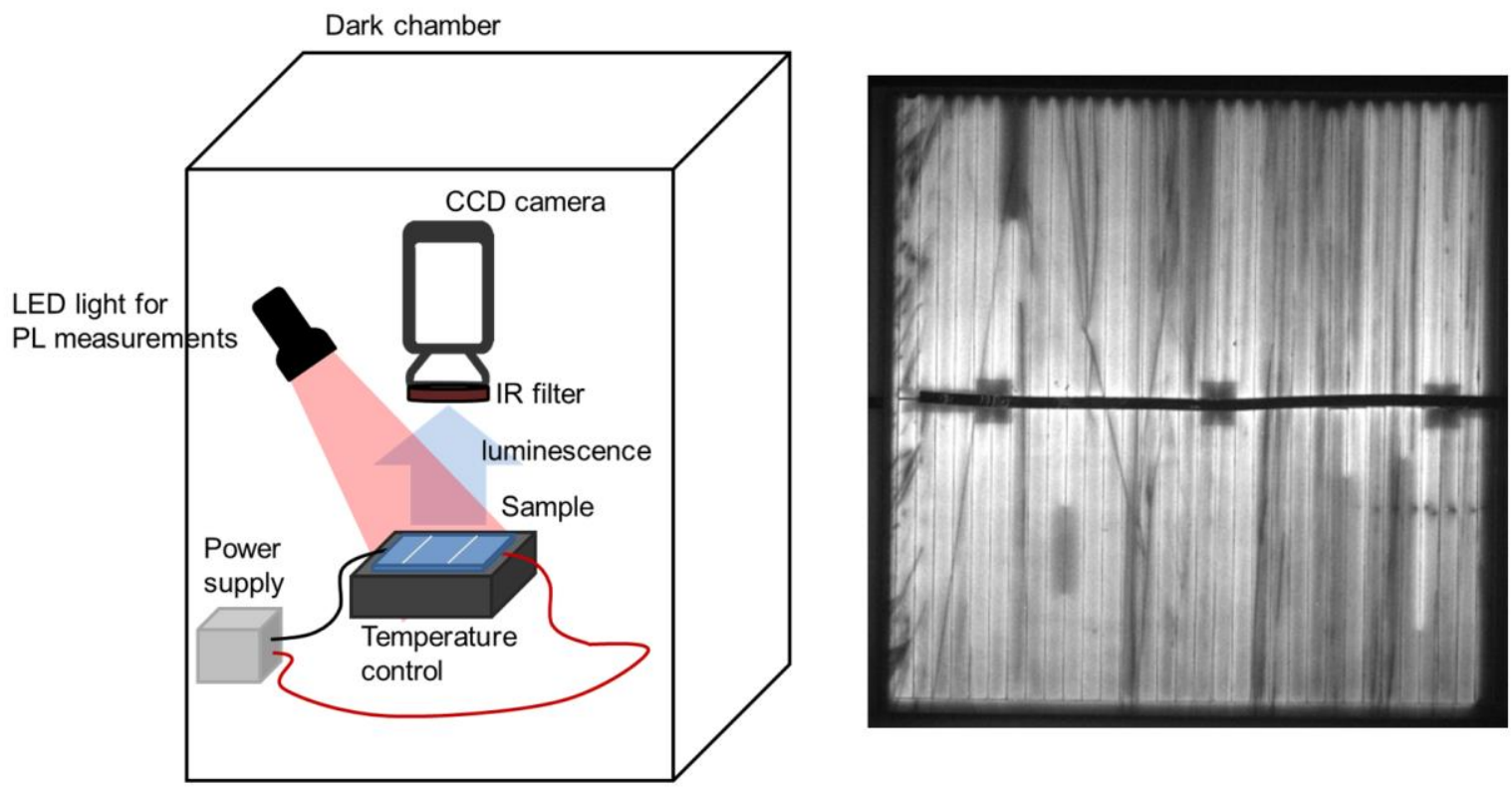

Figure 2.12. On the left, the EL imaging setup at CREST. On the right, an EL image of a ribbon mc-Si PV cell, obtained with the system. Cracks, broken fingers and other defective areas are visible.

The wavelength range of the luminescence excitation signal of crystalline silicon PV devices varies from 950 to $1250 \mathrm{~nm}$, with the peak occurring at approximately $1150 \mathrm{~nm}$. Emission intensity is dependent on the density of defects in the silicon, with fewer defects resulting in more emitted photons, as the minority charge carrier concentration is larger. Methods have been developed to calculate several parameters using EL imaging, such as the diffusion length [112][113][114], the local junction voltage [115][116], and the series resistance of a solar cell [117][118][119]. Degradation mechanisms of PV modules can also be investigated using EL imaging [120]. A method for mapping the apparent absorber bandgap using EL imaging has also been demonstrated recently, with measurements applied on a CIGS PV module [121]. Such parameters can be approximated using a combination of spectral and spatial EL measurements and several assumptions. That is achieved by capturing EL images under different operating conditions and with the use of optical filters that control the 
detectable luminescence spectrum. However, such calculation methods require larger exposure times and multiple images. Still, measurement time is shorter than other spatial characterisation methods.
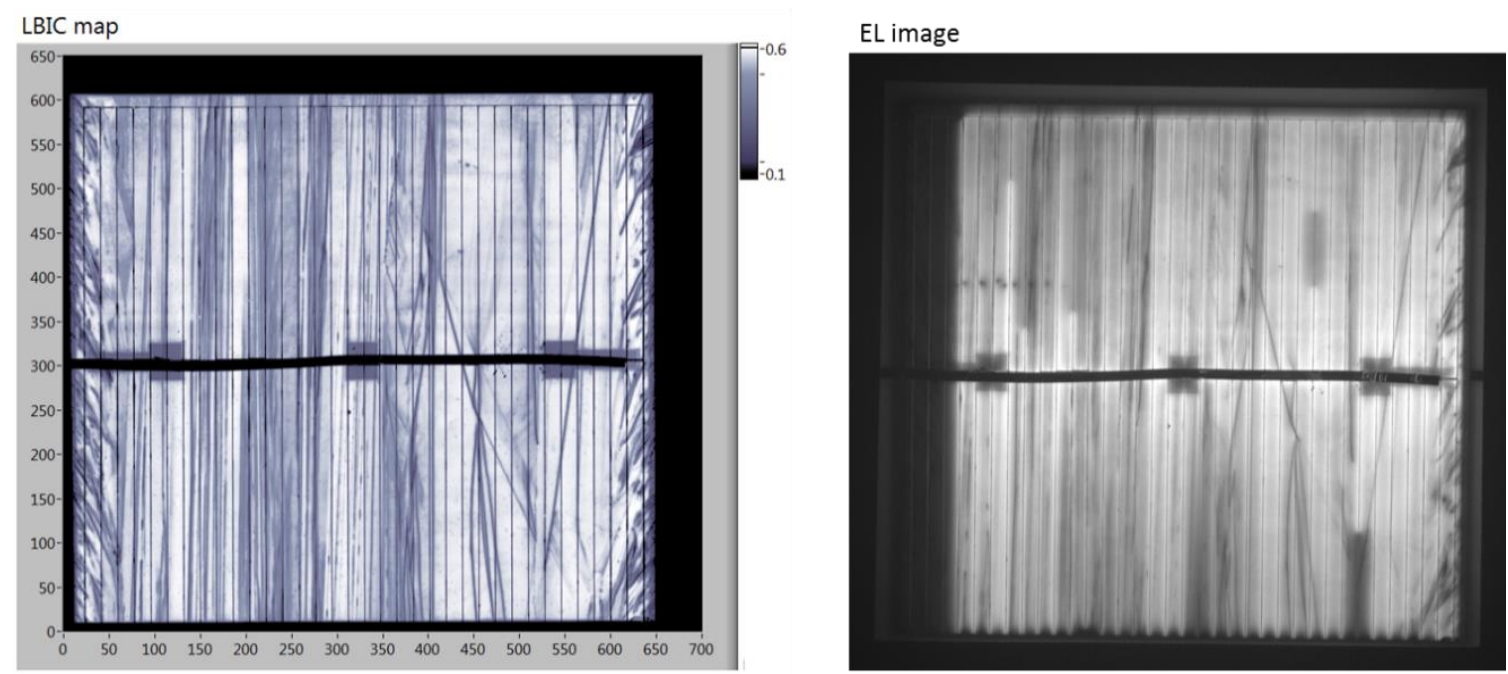

Figure 2.13. On the left, an LBIC current map of an EFG mc-Si solar cell, acquired with the latest LBIC system in CREST, using a laser wavelength of 980 $\mathrm{nm}$. On the right, the EL image of the same sample.

EL provides useful results also for thin film solar cells. EL imaging has been applied and its usefulness has been investigated for CdTe solar cells [122]. A methodology for evaluating the local value of $V_{O C}$ of CdTe solar cells using EL measurements is demonstrated in [123]. A method for calculation of the sheet resistance of the transparent conductive oxide (TCO) layer in thin film PV modules has been introduced recently [124] Moreover, local series resistance analysis of the window and back contact layers can be realised using EL imaging for CIGS modules [40][125]. EL imaging can also be useful for investigating degradation processes of perovskite solar cells [126][127].

\subsubsection{Photoluminescence imaging}

Photoluminescence (PL) imaging also utilises the emission due to recombination of minority carriers in semiconductor devices and captures images of the PV devices using CCD cameras or InGaAs detectors, just as with EL imaging [10]. However, the PV device under test is not excited by forward bias but by illumination with an external light source. In this way, PL imaging avoids physical contact with the sample in most cases, an important practical aspect 
for inline applications in terms of both measurement throughput and reduced risk of mechanical damage. It is also obvious that PL imaging can be applied to every stage of PV manufacturing procedure, from wafers to completed cells and modules. Since in principle the emission is the same as with EL imaging, the emission band is also the same, so it can be detected partially with silicon CCD cameras and almost completely with InGaAs detectors.

PL mapping was initially introduced as a point by point microscopy scanning technique for PV characterisation [128][129]. PL scanning microscopy is still an extremely useful method for investigating material structure and recombination properties of structural defects, especially in thin film devices [130]. Using a confocal configuration, resolution can be significantly increased, which results in an essential tool for material research [89].

$\mathrm{PL}$ imaging with a camera detector was then demonstrated as a very fast technique to measure the minority carrier lifetime with high spatial resolution [131]. Using PL imaging, the effective minority carrier lifetime images of passivated silicon wafers can be acquired at megapixel resolution within seconds. This is orders of magnitude faster than is possible with e.g. microwave photoconductance decay mapping tools [132] that are usually used for measuring lifetime in silicon wafers. Moreover, PL imaging can be useful also for other solar cell manufacturing technologies.

As in the case of EL imaging, there are several device parameters that can be calculated or approximated with the use of PL imaging, especially for crystalline silicon wafers and solar cells. Information about minority charge carrier lifetime can be acquired with PL imaging, with inline application to quality control of silicon wafers [133][134]. Interstitial iron concentrations in multicrystalline silicon wafers can be also imaged using PL [135][136]. The series resistance of finished silicon solar cells can be spatially evaluated, as is demonstrated in [137][138]. Nevertheless, in this case contacting the cell is necessary, as current has to flow over the contacts of the PV device. Imaging the series resistance using PL is preferable to using EL imaging, as PL imaging combined with current flow in the PV device simulates more closely the actual operating conditions. Specific operation points on the IV can be selected, equivalent to the maximum power point. By acquiring several PL images, it has also been demonstrated that a set of performance parameters of the cell such as local power, fill factor and efficiency can be calculated [139].

Most of the time such methods require specific assumptions that do not always hold true. One of the most common being that the light generated current density is uniform across the cell area. Moreover, PL imaging methods need accurate calibration that is 
sometimes sample specific. Lateral variations of minority carrier lifetime can affect calibration procedures and insert errors in measurements [140].

\subsubsection{Lock-in Thermography}

Infrared (IR) thermography is a spatial non-destructive characterization technique that utilizes the radiation in the infrared range of the electromagnetic spectrum (approximately 0.9-14 $\mu \mathrm{m}$ ) to produce images of a specific temperature pattern. Infrared radiation is emitted by all objects and it is proportional to their temperatures, hence, IR thermography makes it possible to determine the surface temperature of a PV device. As steady-state thermography of PV devices shows very poor spatial resolution because of lateral heat spreading in the device, lock-in techniques have been utilized.

The technique of infrared (IR) camera-based lock-in thermography (LIT) was introduced for the first time by Kuo et al [141]. This technique has been further developed and established as a successful spatial characterization technique for solar cells and other electronic devices. The lock-in process requires a periodic signal from the solar cell to be measured. This can be achieved by applying a pulsed voltage to the sample or by illuminating the cell with pulsed light and by measuring the surface temperature modulation with a sensitive infrared (IR) camera. The high cost of such detectors is a disadvantage of LIT. The choice of frequency is a compromise between spatial resolution and detection sensitivity [142]. For low frequencies, lateral heat diffusion makes the acquired images appear blurrier, although the signal is stronger. On the contrary, using higher frequencies improves spatial resolution, but the signal is much weaker. Lateral resolution of thermography images always depends on the diffusion of the thermal waves in the material. The signal-to-noise ratio is determined by the measurement time, that is, the number of lock-in periods used for the measurement. LIT is schematically described in Figure 2.14.

The primary results of a LIT measurement are an in-phase image $\left(0^{\circ}\right.$ image $)$ and an outof-phase image ( $-90^{\circ}$ image). These acquired signals can easily be converted into images of the amplitude and the phase. The phase signal is scaled in degrees expressing the phase shift between the signal and the lock-in reference signal. The amplitude signal cannot be evaluated quantitatively but it provides a good resolution image of local heat source and it is the standard way to display LIT results. 


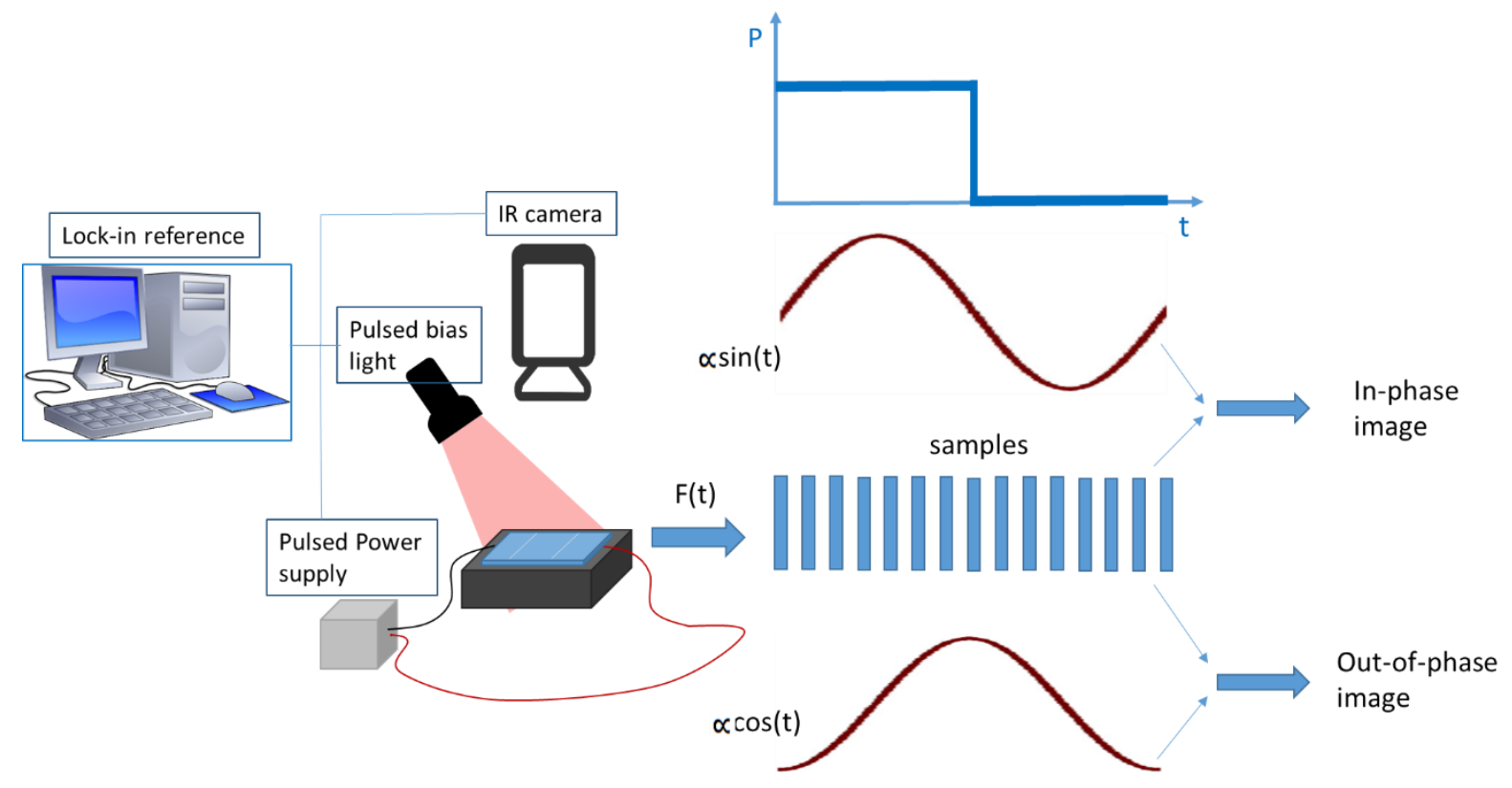

Figure 2.14. Measurement principle of LIT

Due to the dynamic character of the measurement, lateral heat diffusion is limited, resulting in an improved effective spatial resolution. Lock-in thermography is applicable not only for single solar cells but also for solar modules [143]. Initially LIT was developed for application in the dark (DLIT). In DLIT, the modulated bias voltage is applied in the dark and it is the method that was introduced for quantitative evaluation of shunts in solar cells [11]. Illuminated or Light modulated LIT (ILIT or LimoLIT) utilises a modulated light source to provide the pulsed signal, instead of pulsed bias voltage [144]. In this way, a contactless way of applying LIT is provided, although additional voltage bias provides more realistic measurement conditions, simulating actual operating conditions. In similar ways to PL and EL imaging, LIT can evaluate several parameters and features of solar cells. Local series resistance investigations can be realised with i-LIT [145].

\subsubsection{Corescan}

The Corescan (COntact REsistance Scan) method is a destructive method for series resistance measurements in wafer based crystalline silicon solar cells [146][147]. Current is generated locally using a light beam large enough to cover the fingers closest to the scanning point. A probe centred in the light beam is continuously in contact with the surface of the sample, measuring the potential at the front surface of the cell and moves together with the 
light beam over the cell to implement the scan. The probe is made of tungsten and is pressed against the surface by the probe holder weight. This means that the surface is scratched, removing the anti-reflection coating to enable electrical contact with the silicon surface.

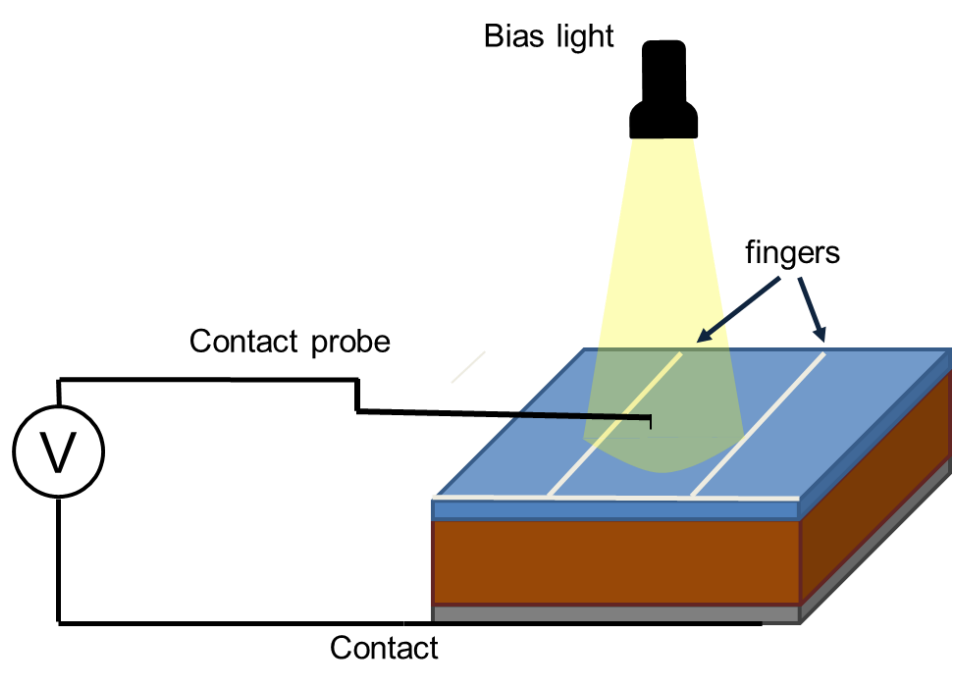

Figure 2.15. Measurement principle of the Corescan method

Effectively, this technique measures the potential difference between a finger of the PV cell and the local point of contact. Using this technique, the series resistance and shunts of wafer based PV cells can be mapped. This becomes useful especially for development and validation of non-destructive measurement methods for series resistance, such as luminescence methods [138][148]. A drawing of the measurement principle of Corescan is presented in Figure 2.15.

\subsubsection{Electron Beam Induced Current (EBIC) measurements}

The EBIC method uses similar principles to LBIC measurements, but instead of scanning the semiconductor area with a light beam, an electron beam provided by a scanning electron microscope (SEM) is used [149]. Classic SEM mapping measurements on PV samples would need the samples to be cleaved and the electron beam would scan the side of the sample as is summarised on the left of Figure 2.16. A typical EBIC measurement system also consists of an SEM and a low noise current amplifier. In this case, the electron beam by the SEM scans the surface of the solar cell, generating electron-hole pairs within the volume that the beam 
penetrates in the cell. The carriers can be collected and amplified, so that a current map is generated, where variations in the generation or recombination of these carriers can be displayed. EBIC imaging is very sensitive to electron-hole recombination and thus it is very useful for determining specific defects that act as recombination centres in semiconductor materials. The general principles of the EBIC method are summarised on the right of Figure 2.16 .
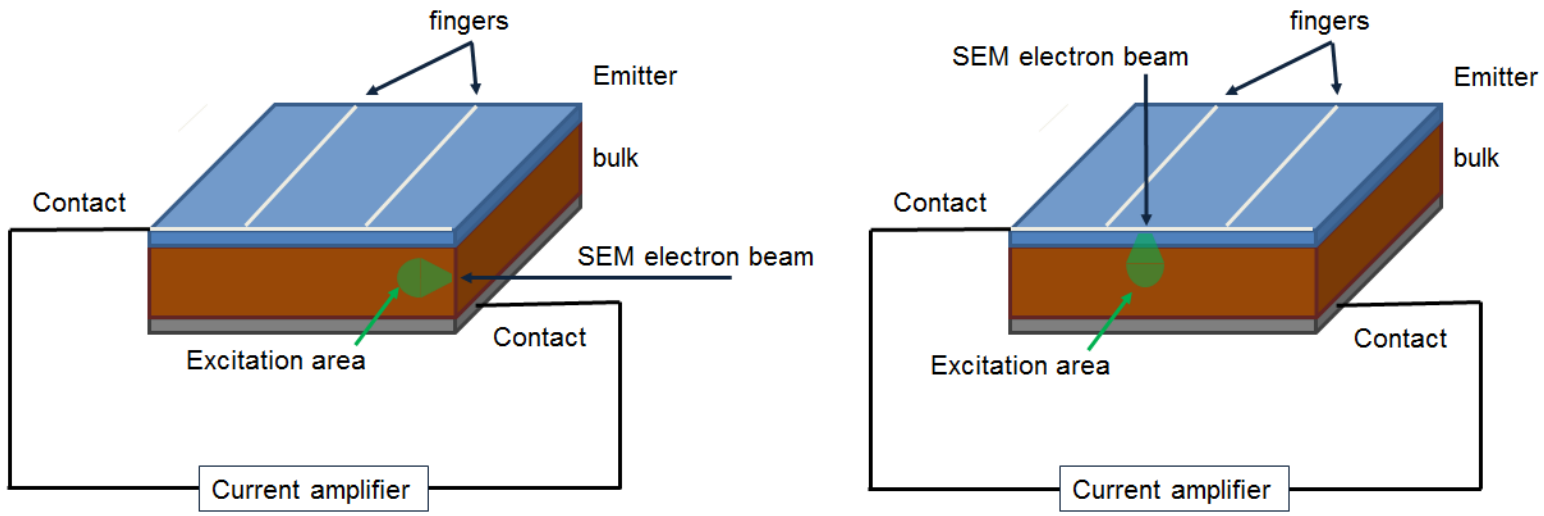

Figure 2.16. On the left, a drawing of the standard way of performing SEM measurements on PV samples. On the right, the measurement principles of the EBIC technique.

EBIC measurements can only be applied to bare cells that are not encapsulated. They have been useful for characterising grain boundaries, dislocations and generally the electrical behaviour of microstructure. EBIC has been used for crystalline silicon[150], although for small areas and not whole cells. The very high resolution it yields and the ability to electrically characterise microstructure, makes it more suitable for characterisation of small research samples such as small CdTe solar cells [151] or organic PV samples [152]. 


\subsection{Conclusion}

As has been described in this chapter, the overall performance of PV cells, and consequently PV modules, can be dominated by local defects and areas of underperformance within the cells. Local features can be inactive areas or can act as recombination centres that reduce the efficiency of PV devices. These spatial features can be production induced, can appear due to ageing of devices or can be just features of specific intrinsic material properties. Different types of PV devices suffer from different types of defects and local features. New emerging technologies such as organic and perovskite solar cells are very unstable and exhibit a large number of defects when aged. For all the above reasons, the development of high efficiency, cost effective solar cells is strongly connected with the development of useful PV spatial characterisation techniques.

In this chapter the established characterisation methods were summarised. It became clear that each of the methods described is more suitable for detecting and investigating specific features. Moreover, some methods are more useful for specific types of solar cells than others. All these methods are not necessarily competing with each other but are complementary, as other authors have also pointed out [153][154][155]. Within this context of spatial characterisation methods, this work focuses on the development of a new current mapping technique, which uses different sampling principles to LBIC measurements, which is the standard current response mapping method. This new development will not only provide an alternative new method for current mapping, but has the potential to significantly reduce measurement time for current mapping of PV devices. 


\section{Chapter 3}

\section{Compressed sensing imaging and application to PV spatial characterisation}

\subsection{Overview}

The novel spatial characterization method that is developed in this work is based on a recently reported data acquisition theory named compressed (or compressive) sensing, introduced by D. Danoho [14] and J. Candès, J. Romberg and T. Tao [156]. This theory has offered a new sampling concept which can become very useful in situations where the classic point by point sampling procedure is very time consuming, impractical or expensive. According to this theory, one can reconstruct a signal from highly incomplete or inaccurate information.

Lossy compression techniques for datasets are widely used in everyday life; MP3 music files are compressed audio files, JPEG compression is used for image files. In such instances, a large dataset is transformed and represented by a different basis. For instance, a dataset can be represented in the Fourier space, using the Fourier coefficients. In the case of JPEG compression for images, the complete dataset (typically several megapixels) of the image is initially available but after lossy compression, only the largest basis coefficients are stored. When reconstructing the signal the non-stored coefficients are simply set to zero. This means 
that on almost all occasions a large part of the information is simply thrown away during the compression process.

In many cases, large effort is spent in order to obtain full information of a signal of $N$ elements and afterwards most of the information is discarded at the compression stage, where only $K$ elements are kept. One might ask whether there is a practical way of obtaining the compressed version of the signal more directly, by taking only a small number of measurements of the signal in the first instance. This can be extremely useful in cases when measurements have a high cost and reducing the number of measurements can efficiently reduce cost and time of the measurement procedure. It is not obvious at all whether this is possible, since directly measuring the largest coefficients requires their location to be known beforehand. Nevertheless, compressed sensing provides a way of constructing a compressed version of the original signal by taking only a small number of linear and non-adaptive measurements. The precise number of required measurements is comparable to the compressed size of the signal, though clearly the measurements have to be suitably designed.

The traditional approach to measuring signals follows the well-known Shannon-Nyquist sampling theorem, which states that the sampling rate must be at least twice the highest frequency of a signal [157]. In many applications, including digital imaging and video cameras, the Nyquist rate is so high that more samples are acquired than are practical to manipulate, making compression a necessity prior to storage or transmission. In other applications, including imaging systems (medical scanners and radars) and high-speed analog-to-digital converters, a higher sampling rate is prohibitive, either because of a high cost per sample, or because specific samplers cannot achieve the high sampling rates required by the ShannonNyquist theorem. Compressive sensing (CS) can potentially overcome these difficulties, demonstrating that certain signals or images can be recovered from what was previously believed to be a highly incomplete measurement set.

In order to have a reduced number of acquired measurements and still adequately describe a signal, its compressibility has to be exploited. The measurements in the CS case are not point samples but more general linear combinations of the signal. An overview of the compressed sensing theory, compressed imaging and its applications in instrumentation development is given in this chapter. At the core of almost all physically realised CS imaging systems is a Digital Micromirror Device (DMD), the properties of which and applications to PV characterisation will be discussed. Finally the general methodological approach of applying CS to current mapping will be described, along with some considerations for this specific application. 

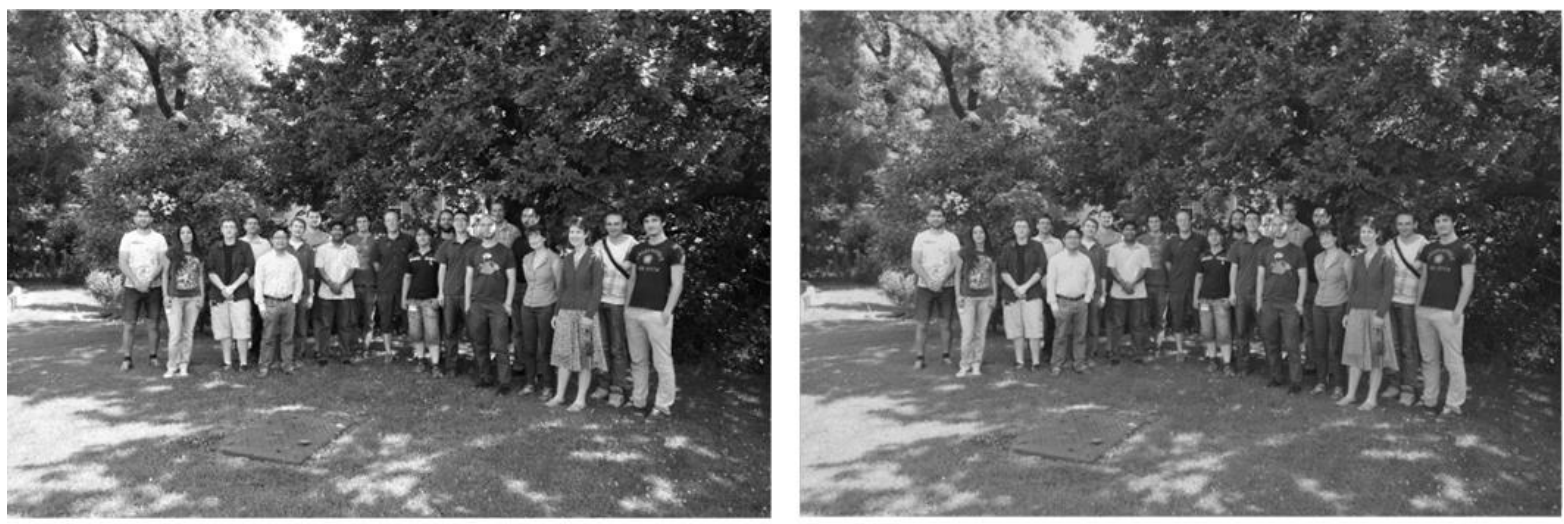

Figure 3.1 A photograph of the APV group in CREST and a reconstructed image of the initial photograph, using only the largest $2 \%$ of the Discrete Cosine Transform (DCT) coefficients.

\subsection{The Digital Micromirror Device (DMD)}

The micromirror elements that are the heart of the DMD are optical micro-electromechanical (MEMS) components and were introduced and patented in 1987 as spatial light modulators [158]. Their usefulness for digital projection applications was proposed some years later [159][160]. After several improvements over time, the micromirror array device design in current use was invented and patented in 1996 [161]. Any display device that is based on a DMD chip for creating the projections is referred to as Digital Light Processing (DLP). DLP technology has been adopted for almost all display applications, such as simple projectors, television sets, and cinema projectors.

\subsubsection{Properties}

The structure of the DMD chip is illustrated in Figure 3.2. The micromirror array area consists of thousands of individual aluminium micromirrors, the actual number depending of the maximum resolution the specific DMD model can provide. Maximum resolution varies from $1024 \times 768$ to $2560 \times 1600$. The size of the micromirror array and the individual micromirrors also depend on the DMD model. The mirror array area is square in almost all cases, with each micromirror having a size of $13.7 \mu \mathrm{m}$ by $13.7 \mu \mathrm{m}$ for the lower resolution 
models and down to $7.6 \mu \mathrm{m}$ by $7.6 \mu \mathrm{m}$ for the high resolution ones. In every case, the distance between micromirrors is approximately $1 \mu \mathrm{m}$. The micromirrors sit on a silicon oxide memory cell, which defines the state ( 0 or 1 ) of each mirror. Mechanical stops limit the micromirror tilt angle to $\pm 10^{\circ}$ depending on the digital state of the underlying CMOS (complementary metal-oxide-semiconductor) memory cell. Thus, each one of the micromirrors can reflect light in one of two distinct directions, depending on if it is set on the "on" or "off" state. This is achieved with the use of a yoke relative to the substrate which enables each micromirror to tilt about a pair of hinges. These components are illustrated in Figure 3.2. The maximum switching rate between states depends on the model of the DMD array and can be as fast as $22.7 \mathrm{kHz}$.

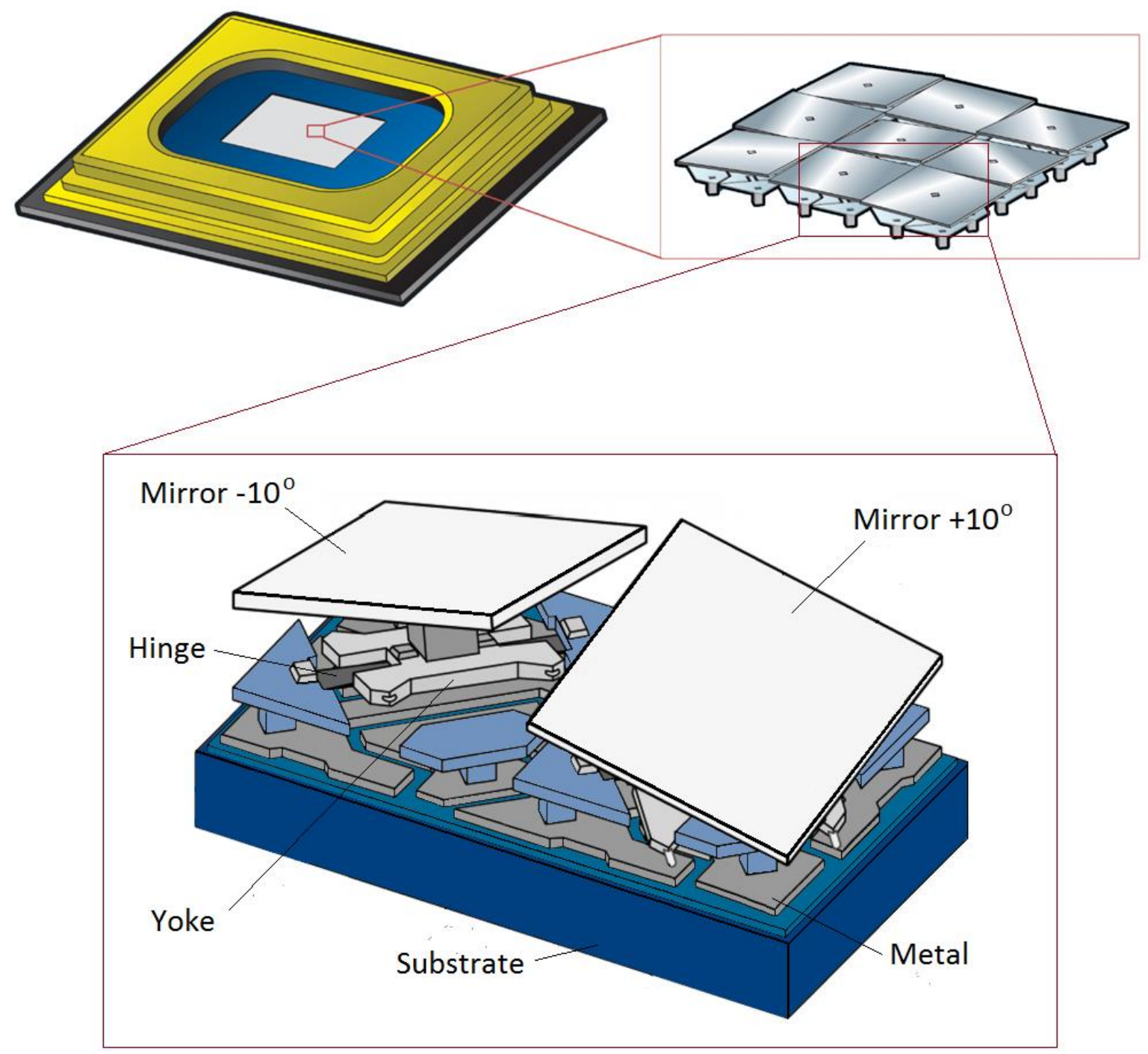

Figure 3.2 Drawing of the micromirror array and details of its specific components. 
The reliability and lifetime range of DMD chips has been extensively tested and it has been demonstrated that their lifetime without degradation can exceed 100,000 hours [162]. Initial durability experiments revealed specific failure mechanisms associated with hinge memory and surface contamination [163][164]. The problem of hinge memory lifetime has the impact that a micromirror after a long period of use that specifically lands the pixel on one side more often than the other, the micromirror may not return its exact actual position. Contamination of the surface of the micromirror array area with foreign particles can lead to difficulty of movement and even damage to the micromirrors. After 100,000 hours of operation, calculated MTBF (mean time between failures) has been reported to be higher than 650,000 hours [162]. DMD chips have shown robustness under extreme environmental conditions of temperature and light intensity.

\subsubsection{Application to PV characterisation}

Apart from the method that is described in this work, there are only a few cases where DMD chips have been used directly or indirectly in instrumentation for characterisation of PV devices. The first time a DMD chip was used for PV characterisation was a tomographic line scan setup for current mapping of PV devices reported in [165]. This was also the first time that a pattern projection sequence (line scan) was adopted instead of a point by point scan for current mapping. Current mapping measurements of a solar cell using a DLP projector were also reported in [166]. The spatial resolution achieved was approximately $1.5 \mathrm{~mm}$.

DMD use has also been proposed for building small scale solar simulators or solar cell spectral response characterisation layouts. By utilising a super continuum laser and a DMD, a small scale solar simulator useful for PV characterisation can be realised, that can reproduce very accurately the AM1.5 spectrum [167]. This is achieved by combining a prism or grating and a DMD to create an optical filtering procedure that can accurately control the output spectral radiance of the system. In the same way, a spectral response measurement system can be built [168]. Frequency modulation of the micromirrors in such a system can result in a decrease in spectral response measurement times by a factor of 500 [169]. The work of this thesis adds one more application of the DMD chip to PV characterisation. 


\subsection{Compressed sensing}

\subsubsection{Summary of compressed sensing theory}

Following the usual sampling process for measuring a signal $\boldsymbol{x}[n], n=1,2, \ldots \mathrm{N}$, a potentially large number of samples $N$ have to be acquired. This is the case even when the data will be compressed to a $K$ element vector, where $K<<N$. After acquiring $N$ measurements, the system must compute all of the $N$ transform coefficients, even though it will only keep the values and locations of the $K$ largest elements needed for an accurate representation in the transform domain (compressed form) and discard the rest. The purpose of compressive sampling is to directly measure these $K$ coefficients, applying $M<<N$ number of measurements, where $K<M$. Since the locations of the $K$ coefficients are not known, one has to apply a slightly larger number of measurements $M>K$ to ensure that the $K$ coefficients are captured.

The signal to be sampled is a real-valued, finite-length, one-dimensional, discrete-time signal $\boldsymbol{x}$. It is represented as an $N \times 1$ column vector in $\mathrm{R}^{N}$ with elements $\boldsymbol{x}[n], n=1,2 \ldots, N$. Any image can be represented as a long, one-dimensional vector. For instance, an image of size $\sqrt{N} \times \sqrt{N}$ pixels can be described by an $N \times 1$ vector. Using compressed sensing, a compressed representation of a signal, $\boldsymbol{x}$, is acquired using $M<N$ linear measurements between $\boldsymbol{x}$ and a set of test functions $\left\{\varphi_{m}\right\}_{m=1}^{M}$, forming $\boldsymbol{y}[m]=\left\langle\boldsymbol{x}, \varphi_{m}\right\rangle$ in which $\boldsymbol{y}$ is the actual measurement. Stacking test functions $\left\{\varphi_{m}\right\}_{m=1}^{M}$ as rows in an $M x N$ matrix $\Phi=\left[\varphi_{1}, \varphi_{2}, \varphi_{3}, \ldots \varphi_{m}\right]$, the problem can be written as [170]:

$$
\boldsymbol{y}=\Phi \boldsymbol{x}
$$

In general there is loss of information as a result of the transform from $\boldsymbol{x}$ to $\boldsymbol{y}$, as $\boldsymbol{y}$ has significantly fewer dimensions than $\boldsymbol{x}$. Since $M<N$, there are infinitely many $\{\boldsymbol{x}: \boldsymbol{y}=\Phi \boldsymbol{x}\}$. This is an underdetermined problem with infinite solutions. However, a sensing matrix $\Phi$ can be designed such that an almost exact approximation of signal $\boldsymbol{x}$ can be recovered from measurement $\boldsymbol{y}$, if $\boldsymbol{x}$ is sparse or compressible. A signal being sparse means that almost all of its elements are zero. In practice, few real-world signals are truly sparse, although almost all of them are compressible, meaning that they can be well-approximated by a sparse signal, or are sparse after a transform [171]. 


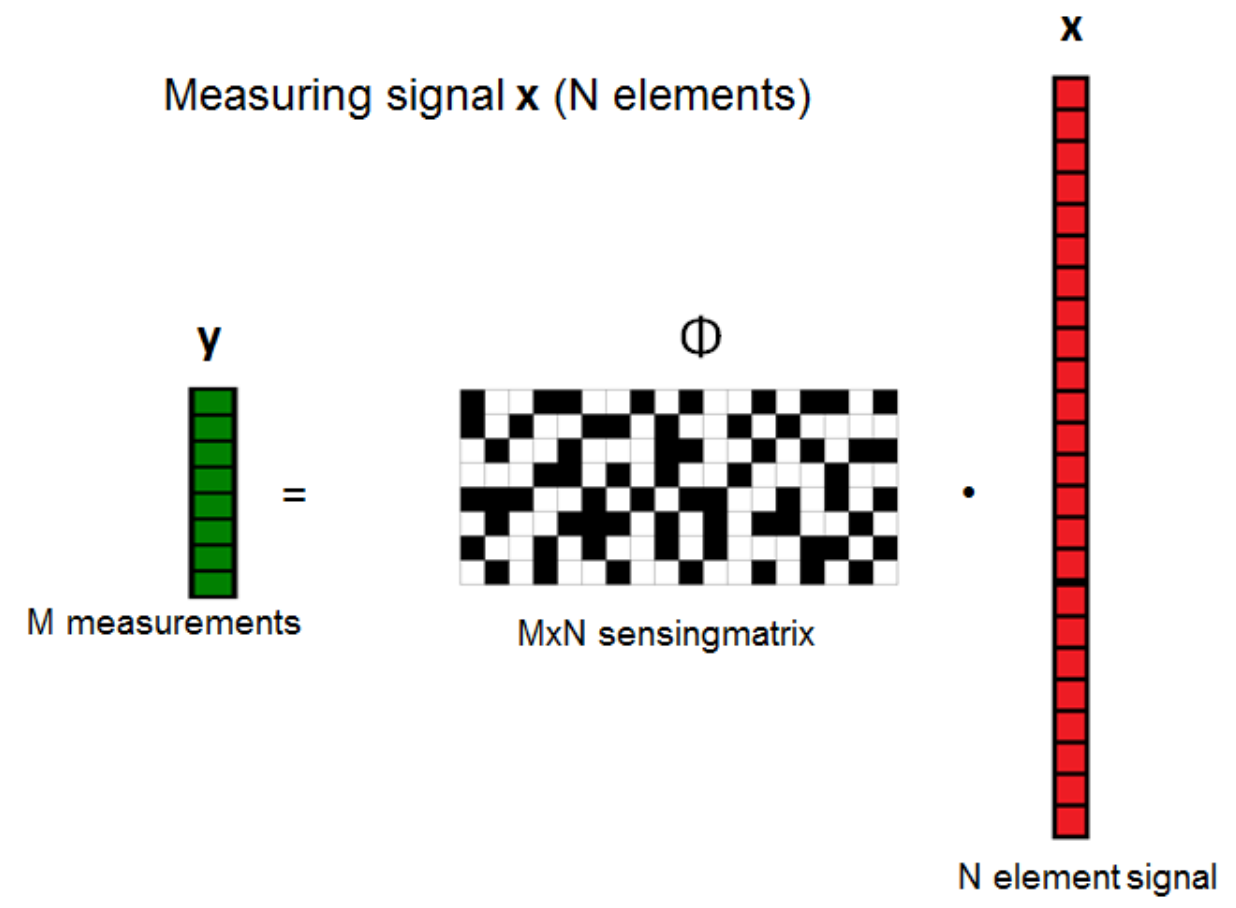

Figure 3.3. Sampling a signal of $N$ elements with an $M x N$ sensing matrix results in the measurement vector consisting of $M$ elements.

The sensing matrix $\Phi$ should have some specific properties so that a successful CS process can be achieved [172]. As mentioned above, real signals are compressible, which means they have a sparse representation after a transform, $\Psi$. When $\Psi \in R^{n x n}$ is a representing basis (or dictionary), the sparse version of $\boldsymbol{x}$ is $\boldsymbol{\alpha}$, satisfying

$$
x=\Psi \alpha
$$

It has been shown that in order to achieve successful CS measurements, a matrix $\Phi$ must be chosen having minimal coherence with $\Psi$ [170]. In other words, a matrix $B=\Phi \Psi$ is desired to have columns with the smallest possible correlations. A second important property is that $\Phi$ must satisfy the Restricted Isometry Property (RIP) [173]. A matrix $\Phi$ satisfies the RIP of order $\mathrm{k}$ if there exists a $\delta_{k} \in(0,1)$ such that [171]:

$$
\left(1-\delta_{k}\right)\|x\|_{2}^{2} \leq\|\Phi x\|_{2}^{2} \leq\left(1+\delta_{k}\right)\|x\|_{2}^{2}
$$

If a matrix $\Phi$ satisfies the RIP of order $2 k$, then it is ensured that $\Phi$ approximately preserves the distances between any pair of $k$-sparse vectors. This is especially important for signal recovery when measurements are contaminated with noise. 
In practical applications it is difficult to test if a sensing matrix satisfies the aforementioned properties. In most cases, $\Phi$ would have thousands of rows and columns. However, both the RIP and incoherence can be achieved with high probability simply by selecting $\Phi$ to be a random matrix [174]. Several types of matrices have been used in CS applications; Gaussian random matrices [14], scrambled Fourier (SF) matrices [156], binary orthogonal transform matrices [175] and sparse matrices generated from block diagonal matrices, such as the scrambled block Hadamard (SBH) matrix [176]. As mentioned above, even scrambled random binary matrices work with high efficiency, as long as they are highly sparse [177]. For applying compressive sampling, the matrices do not have to be binary (ones and zeroes) but can have any values, as long as they possess the necessary incoherence with the representation basis and that they satisfy the RIP.

The signal reconstruction algorithm must take the $M$ measurements in the vector $y$, the random measurement matrix $\Phi$, the basis $\psi$ (transform) and reconstruct the $N$-length signal $\boldsymbol{x}$ or, equivalently, its sparse coefficient vector $\boldsymbol{\alpha}$, as $\boldsymbol{x}=\Psi \boldsymbol{\alpha}$ and $\boldsymbol{y}=\Phi \boldsymbol{x}=\Phi \Psi \boldsymbol{\alpha}$. Among a large variety of algorithms in the literature, the $\ell 1$ minimisation algorithm is mostly preferred in this work. The reasons are the simplicity of the algorithm, its well-characterised performance and the open access software packages available. It has been shown that using the $\ell_{1}$ minimisation optimisation one can exactly reconstruct $k$-sparse vectors and closely approximate compressible vectors stably with high probability using a small number of random measurements [14]. In other words the solution to the underdetermined problem is the $\boldsymbol{x}$ vector (or more precisely the $\alpha$ vector) with the minimum $\ell_{1}$ norm [174]:

$$
\widehat{\boldsymbol{x}}=\operatorname{argmin}\|\boldsymbol{x}\|_{1} \text { subject to } \Phi \boldsymbol{x}=\boldsymbol{y}
$$

Or

$$
\widehat{\boldsymbol{\alpha}}=\operatorname{argmin}\|\boldsymbol{\alpha}\|_{1} \text { subject to } \Phi \Psi \boldsymbol{\alpha}=\boldsymbol{y}
$$

This problem is called basis pursuit and can be reduced to a linear problem [14][170]. There are many algorithms available in the literature, as well as open access code for their solutions. In this work the main algorithm used is the primal dual $\ell_{1}$ minimisation, included in the open access " $l_{1}$ magic" toolbox in MatLab, which is written by Justin Romberg [156]. Other methods that were also examined in this work are the SPGL1 algorithm (spectral projected-gradient for $\ell_{1}$ minimisation) [178] and the Danzig selector algorithm, included in the " $\ell_{1}$ magic" toolbox [179]. Additionally, the $\ell_{1}$ minimisation algorithm can also be altered to take into account measurement noise ( $\ell_{1}$ denoise). 
Apart from the $\ell_{1}$ minimisation, other algorithms were also investigated in this work, in order to determine the most suitable for this specific application. "Greedy" algorithms exist for CS reconstruction. Greedy algorithms choose the locally optimal solution at each iteration with the hope of finding a global optimum. A greedy strategy does not in general result in an optimal solution, but may yield locally optimal solutions that approximate a global optimal solution in reasonable time and accuracy. Among the available greedy algorithms for CS, the Orthogonal Matching Pursuit algorithm (OMP) [180] was used, as it can be more useful in very specific cases, as will be demonstrated in the next chapter. The Compressive Sampling Matching Pursuit (CoSaMP) algorithm [181] and the Total Variation (TV) minimisation algorithm (included in the " $\ell_{1}$ magic" package) were also tested. More than 40 open access toolsets for CS reconstruction were found in the literature. This means that an evaluation of all the available software is not possible and also not within the scope of this work. Additionally it is outside the aim of this work to build a new algorithm or code one of the existing methods from scratch. Nevertheless, among the algorithms explored here, the $l_{1}$ primal dual algorithm provided the most reliable and accurate solutions, as will be illustrated in the next chapter.

\subsubsection{Compressed imaging systems}

For building a CS imaging system, there are several important considerations to bear in mind. The design should be a compromise between physical considerations, such as size and cost, reconstruction accuracy and reconstruction speed. One of the first examples of a CS imaging system was the Rice single-pixel camera [182], where a single pixel detector is used to image a scene. In this single pixel camera system, the scene of interest is projected on the DMD pattern generator, like it would be projected on the pixel detector array of a CCD camera. The DMD inserts the binary patterns and the total intensity of each projection is measured with the single detector. Since the individual orientations of the micromirrors in the DMD can be altered very rapidly, a series of different binary random projections can be measured successively in a relatively short period of time. The original image is then reconstructed from the resulting observations using the techniques described in the previous section. Any binary projection matrix can readily be implemented in such a system, so that existing CS theory can be directly applied to the measurements. The main disadvantage is that the camera has to be focused on the object of interest until enough samples have been collected for reconstruction. This also means that the time required may be prohibitive in some applications. 
This initial CS imaging demonstration with the single pixel camera reflects the general measurement principles that CS imaging systems follow. In all systems the sampling is achieved using a sensing matrix consisting of the binary projection sequence that is inserted in the system by the DMD. Any CS imaging system will have to rely on an encoding device that inserts the patterns/test functions into the sampling procedure. The two cases of CS imaging system approaches are presented in Figure 3.4. The approach adopted in this work is the one illustrated on the left of Figure 3.4. A collimated beam overfills the DMD, which creates the patterns. With suitable optics, the sequence of patterns is projected onto the sample, in our case a PV device. In the second case, the sample or the object to be observed is excited/illuminated and the DMD is actually spatially encoding the image with the pattern sequence just before a detector measures the overall signal, as is illustrated on the right of Figure 3.4.
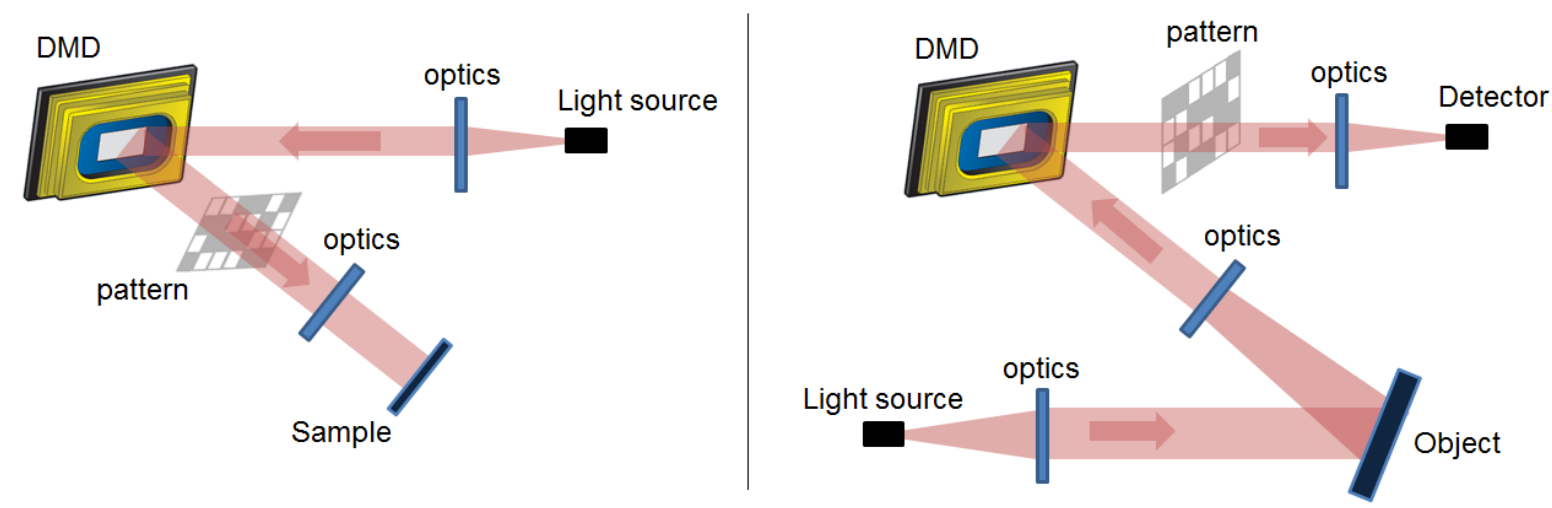

Figure 3.4. CS imaging experimental layout approaches mainly used in CS applications.

This latter system architecture is extremely useful for applications in which the detectors are expensive and building a high resolution multi-pixel camera has a prohibitive cost. Adopting the CS imaging approach, systems that can provide spatial information can be realised based on just a single pixel detector. This feature has been highlighted to have great potential for applications in astronomy [183]. There are other specific cases where data are already encoded, incomplete or are compressively sampled without the need of an external encoder such as the DMD. In such cases the application of compressive sampling is even more attractive for successfully reconstructing spatial information. Another significant feature that is specifically essential for this work is that using compressive sampling, the number of measurements that a system has to acquire in order to provide an estimation of a signal can 
be significantly reduced, to something much smaller than the number of elements of the reconstructed signal.

\subsubsection{Applications in spatial characterisation instrumentation}

The first application of CS was in the medical domain. One of the first CS systems demonstrated was CS Magnetic Resonance Imaging (MRI) [16][184]. CS has the potential to reduce the total scan time and cost of $\mathrm{MRI}$ acquisitions and consequently to minimise the burden of imaging to patients. This can lead to greater patient throughput, reducing the cost of $\mathrm{MRI}$, or permit more accurate imaging to be completed per unit time. Another medical imaging application of CS that has been proposed is CS x-ray computed tomography, which can potentially reduce the x-ray dose to patients, due to the reduction of sampling requirements [185]. Apart from medical applications, CS applications in radar systems have also been presented, in order to reduce data size, complexity, instrument weight, power consumption and system cost [186][187]. Similarly, ground penetrating CS radar systems have been proposed [188].

Recently CS has been proposed as a more efficient way of sampling in hyperspectral imaging applications. Compressive sampling has been demonstrated for fluorescent microscopy, with the same system being able to provide spatial as well as spectral information of biological samples [189]. Utilising CS in imaging instruments is a very convenient way of adding an extra dimension. Projecting the series of patterns while measuring the spectrum for each pattern, maps for each wavelength can be produced. This makes the acquisition of spatial and spectral information of samples possible [190][191]. Due to this reason the implementation of compressed sensing Raman imaging has been proposed, with the actual optimal configuration of such an instrument still being under research [190][192].

CS microscopy instruments have been proposed and demonstrated in different applications and adopting different approaches. Applying compressive sampling to holographic microscopy shows that such a data acquisition strategy is robust to high noise levels and can significantly reduce measurement time [193]. Compressive confocal microscopy has been demonstrated, with the significant advantages of increased signal levels and the fact that the spatial filters necessary for the typical confocal configuration can be omitted [194]. This is achievable due to the double operation of the micro mirrors of the DMD as a pattern generator as well as pinholes for spatial filtering. Compressive sampling has also 
been proposed for surface morphology characterisation measurements [195], electron tomography [196] and atomic force microscopy [197].

Apart from CS MRI, almost all other CS applications for spatial characterisation instrumentation are at a preliminary stage. There is a large number of reconstruction algorithms reported in the literature and more are being developed constantly. Its complexity, especially regarding the reconstruction process, is also a factor that hinders its adoption and development for further spatial characterisation applications. Moreover, the high prices of the DMD chips until recently were also an extra aversive factor, although prices have dropped significantly lately, with the utilisation of DMD chips in DLP projectors and televisions. Due to this last reason, more applications of CS are expected to appear in the near future, the techniques developed in this work being one of them.

\subsection{Applying compressed sensing to PV current mapping}

\subsubsection{General approach}

It has been made clear that the application of compressed sensing in spatial characterisation instrumentation can lead to some significant advantages; a lower number of measurements needed for acquiring a measurement map and increased signal levels. Consequently, CS can also prove useful for current mapping of PV devices and the principles of this approach are presented in this section.

The standard approach of current mapping that conventional LBIC systems use is a point by point scan of a laser beam across the sample. The current response is measured at the device electrical terminals or contacts for each laser point position, in order to produce the current map. For the application of CS for current mapping, sampling has to be achieved using the test functions in the sensing matrix. These test functions are in reality random binary patterns. This sequence of patterns has to be projected on the cell so that compressive sampling can be achieved. The sampling procedure is illustrated in Figure 3.5. The patterns are expressed as rows in the binary sensing matrix $\Phi$. The sample under test is illuminated with the sequence of binary patterns, and the current response is measured for each pattern, thus populating measurement vector $\boldsymbol{y}$. 
Following this procedure, the measurement vector $y$ is acquired, its number of elements depending on the level of undersampling; for 50\% undersampling, $\boldsymbol{y}$ has half the elements that the final current map vector has (the number of image pixels in that latter case). The sensing matrix $\Phi$ is known by construction; in this work uniformly distributed random patterns are used as test functions. Equation (3.4) can be then used to acquire the current map in vector form $\boldsymbol{x}$. In practice, the sensing matrix is transformed using the Discrete Cosine Transform (DCT) before being inserted in the reconstruction algorithm. In this work the transformed sensing matrix is inserted into one of the available CS reconstruction solvers in MatLab, along with the measurement vector $y$. For the $\ell 1$ reconstruction algorithm, equation (3.5) is used and the output of the reconstruction algorithm is vector $\boldsymbol{a}$. The inverse DCT provides the result, which is current map $\boldsymbol{x}$.

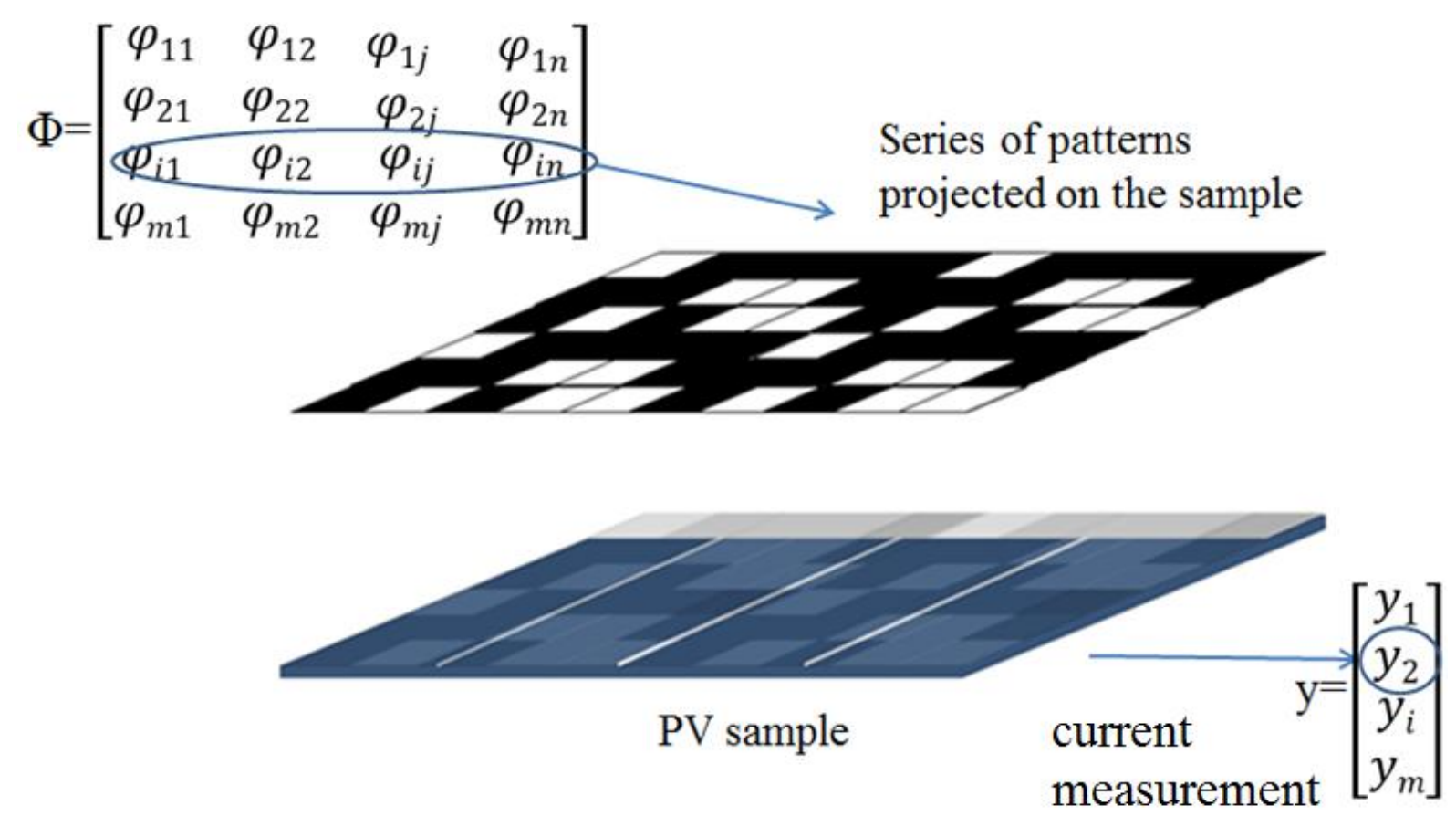

Figure 3.5. The compressive sampling procedure for PV current mapping; the binary patterns in sensing matrix $\Phi$ are projected on the PV device under test. For each pattern, the current response is measured, populating measurement vector $\boldsymbol{y}$.

The compressive sampling and reconstruction procedures are realised in different steps and are not embedded in the same software throughout this work. The flowchart of the overall process is illustrated in Figure 3.6. 

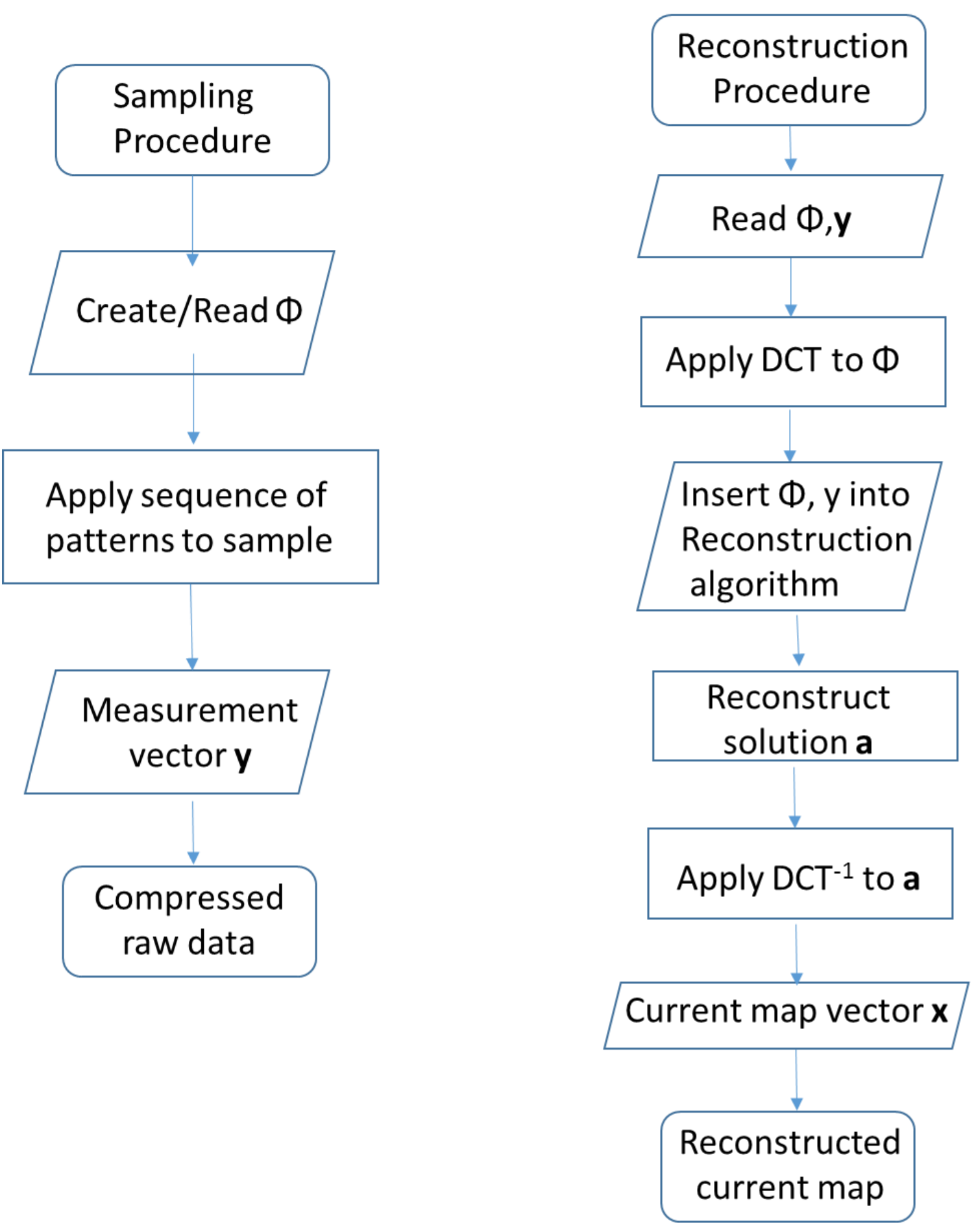

Figure 3.6. Flowchart of the CS current mapping procedure that is used in this work

The compressive sampling software is implemented in LabView in all cases, with an extra step in $\mathrm{C}++$ in the simulations case, as will be described in the next chapter. The sensing matrices are always constructed in LabView before the sampling procedure, the DCT transform is also applied in LabView. The reason is that the hardware control is done with 
LabView for the experimental implementations of this work, so the initialisation of the system and final representation is implemented in LabView. A MatLab script is used in LabView to call the functions for reconstructing, so the user actually never uses MatLab directly. The straightforward implementation of transforms with LabView with its internal functions is also a useful feature that has been exploited.

The reconstruction is an offline process, which means that while a set of measurements is being processed, another sampling process can begin. This is extremely practical as it allows the reconstruction of a set of data which in realistic use can require around a minute, while more measurements are being acquired. Moreover, the reconstruction software produced in this work is universal for all sampling occasions. In detail, the raw data $(\Phi, y)$ are inserted once again into LabView, which applies the DCT transform to $\Phi$ and calls the chosen MatLab algorithm with a MatLab script within the LabView environment. The inverse DCT is then applied to solution $\boldsymbol{a}$ of equation (3.5) in LabView to yield the actual current map $\boldsymbol{x}$.

\subsubsection{Resolution considerations}

In any kind of numerical analysis, the time required for reconstruction depends on the number of points to be used and in many cases increases exponentially. Given a specific final resolution of the current map, it must be verified that for the application in this work the reconstruction process is achieved within realistic time limits. One needs to make certain that the overall measurement time, i.e. the time required for acquisition and reconstruction, is acceptable for the intended purpose. This was investigated the very beginning by utilising an electroluminescence (EL) image. This type of image was chosen as it can be easily acquired and local current measurements have a similar structure. Thus, such an image provides a realistic test-case. Four cases of different levels of resolution were examined; $40 \times 40,60 \times 60$, $80 \times 80$ and $100 \times 100$ pixel images, all presented in Figure 3.7. 

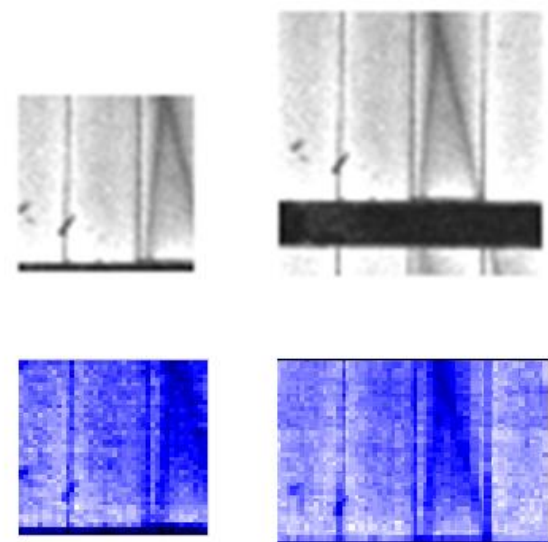

(a)

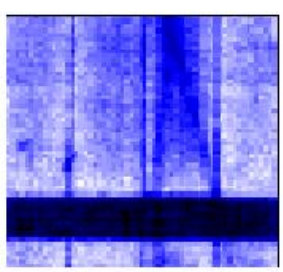

(b)
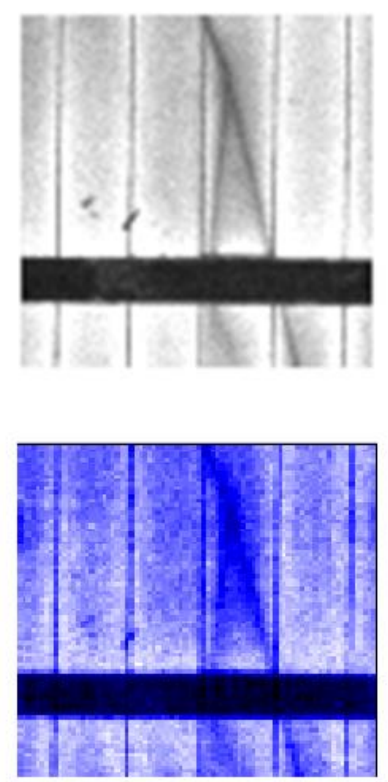

(c)
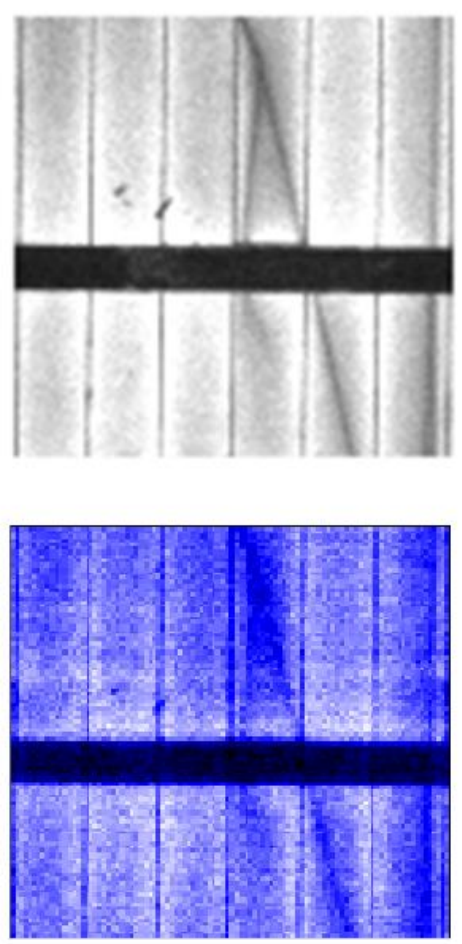

(d)

Figure 3.7. The four parts of an EL image used for the investigation of the impact of the measurement resolution on the reconstruction process (Upper row) and virtual CS measurements (bottom row), with 50\% of measurements used for reconstruction: (a) $40 \times 40$ pixels, (b) $60 \times 60$ pixels (c) 80x80 pixels (d) $100 \times 100$ pixels.

The images were digitised and expressed as matrices, with the values of their elements depending on the EL signal of the equivalent pixels of the image. The procedure for applying CS sampling illustrated in Figure 3.5 was followed. To achieve that, the image matrices were multiplied by the necessary patterns of the sensing matrix, realising a virtual compressive sampling procedure. This procedure was also used as a test process to validate that the available algorithms and the constructed sensing matrices actually work as they should.

The reconstruction time using the $\ell 1$ minimisation algorithm was recorded for each case and the results are presented in Figure 3.8. Most algorithms used in this work exhibit convergence speed of the same order of magnitude, with the $\ell 1$ being the fastest among the ones investigated. The performance of different algorithms is evaluated in more detail in the next chapter. Measurements in the graphs are presented as a percentage, expressing the ratio of the measurements acquired (patterns virtually projected on the sample) to the total number of pixels of the final current map. A conventional point by point method would require as many measurements as the total number of pixels. As expected, higher resolution 
current maps will require more time for reconstruction. In this work a resolution of $100 \times 100$ pixels will be used in most cases, since the time constraints for the reconstruction process are now well defined. An Intel i5, 3.4 GHz, $28 \mathrm{~GB}$ of RAM is used in this work. A maximum of around 4 minutes is observed in the case of the acquisition of $90 \%$ of measurements. However, as will be shown in the following chapters, a 50\% measurement acquisition is enough for an almost exact approximation of a sample's current map. The time required for such a reconstruction is approximately one minute. Since the procedure is an offline process, this time is not directly added to measurement time, as a new set of measurement can start while the previous one is still processed. This last feature proved extremely useful throughout this work and it can be crucial for actual future applications.

For the purpose of analysing each case of different resolution levels, the numerical root mean square error (NRMSE) was calculated, as a function of the number of measurements acquired for reconstruction. The NRMSE for each case is presented in Figure 3.9. It is clear that the higher the resolution, the faster the optimisation algorithm converges and provides a solution. High resolution current maps will converge slightly faster in terms of number of measurements. Fewer measurements can be applied, thus saving considerable time in actual measurements. This effect however, saturates as resolution increases.

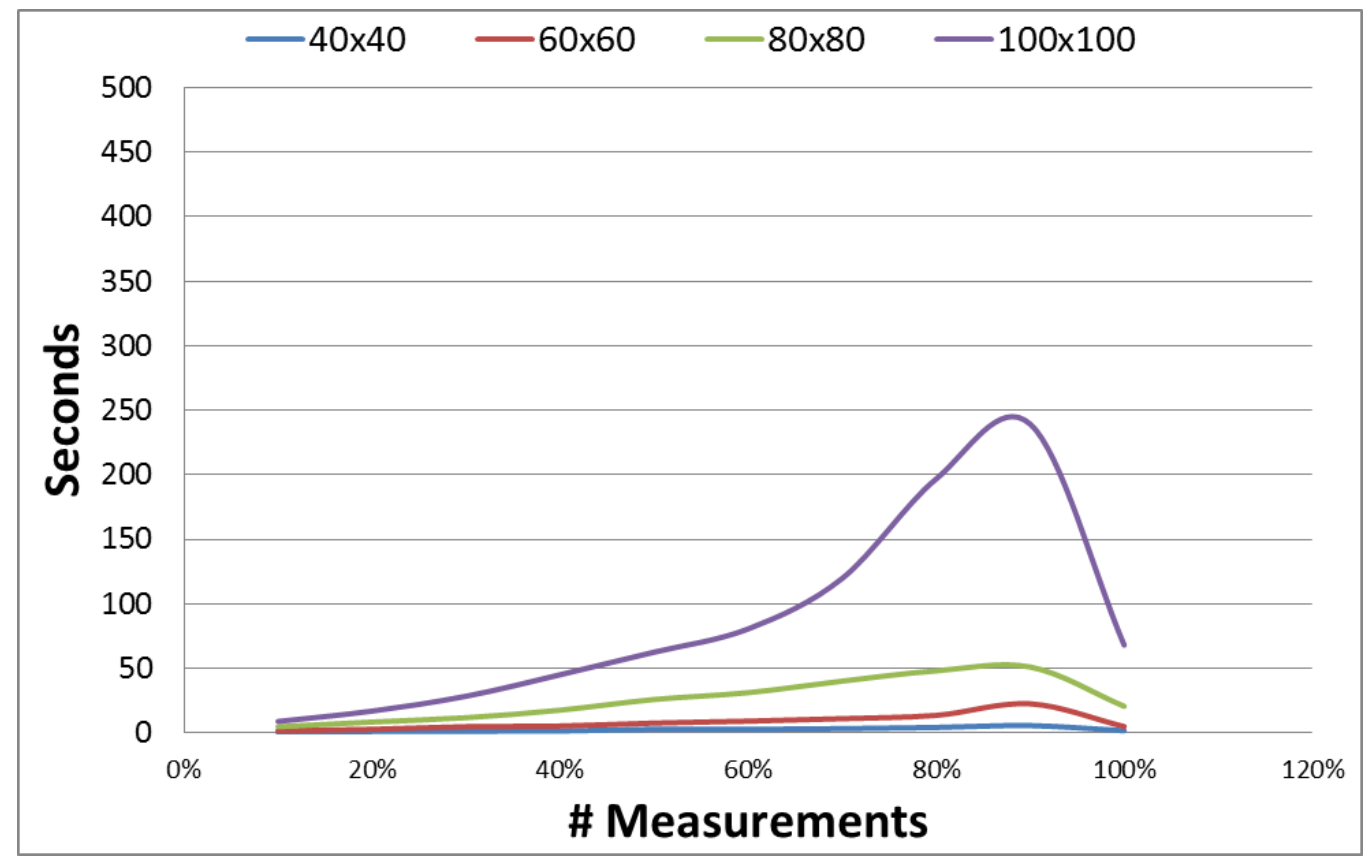

Figure 3.8. Reconstruction time in seconds for four different resolution cases. Numbers of measurements used for reconstructing the current map are presented as percentages of the total final image pixel count. 


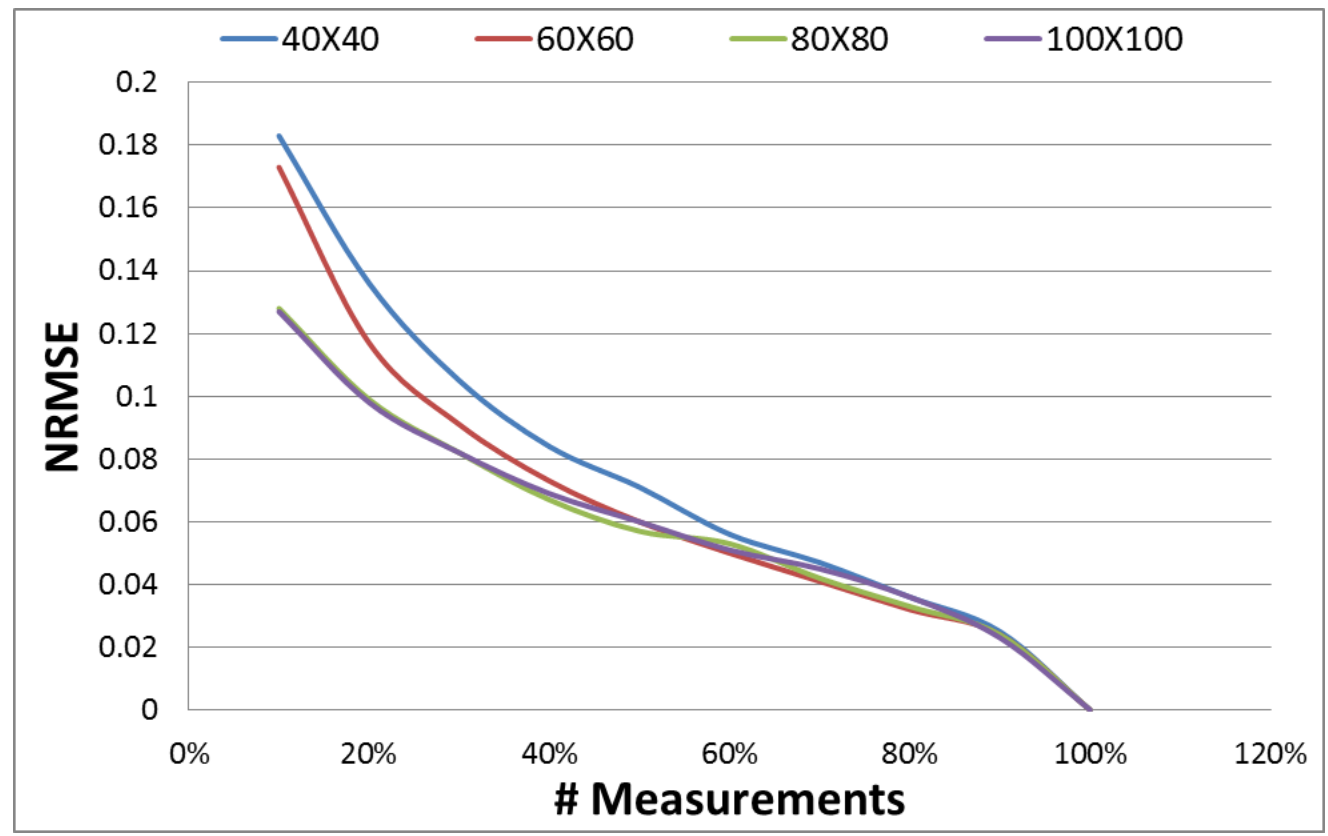

Figure 3.9. NRMSE of four different resolution cases. Numbers of measurements used for reconstructing the current map are presented as percentages of the total final image pixel count.

\subsection{Conclusions}

Compressed sensing theory was summarised in this chapter and some of its applications to spatial characterisation were presented. The DMD chip is an essential part of most experimental applications when compressive sampling is used and so its features were briefly explained. The CS current mapping procedure that is developed in this work for PV device characterisation and its properties were described in detail. These procedures will be used throughout this work. Some insights for the best practices to use were gained from previous CS applications to instrumentation reported in the literature. The $\ell 1$ minimisation algorithm will be mostly used for reconstruction throughout this work, although other algorithms will also be tested. This choice is made as this algorithm has also been tested and used in other CS applications, its performance is well known, it is open access and straightforward to implement. Nevertheless, additional algorithms are used and evaluated in the following chapters. 
The investigation of CS current mapping is realised using electrical spatial simulations as well as experimentally. Regarding the experimental case, a DMD chip is utilised in any case, either directly or indirectly; in the first case, a prototype optical setup will use the DMD directly for generating the necessary patterns; a second approach is to use a commercial projector in order to apply the necessary patterns, indirectly using the DMD integrated within the projector. In the following chapters the potential of CS current mapping will be explored through these implementations, applying the principles already presented. 


\section{Chapter 4}

\section{Spatially Resolved Electrical Simulations of Compressed Sensing Current Mapping}

\subsection{Overview}

Spatial variations of solar cell parameters are crucial for understanding device performance. Spatial variability of generated photocurrent densities in PV devices and even small local flaws can have very significant impacts on overall device performance. Even in ideal cases, the local photocurrent density is not perfectly uniform across a PV device. As was highlighted in the previous chapters, current mapping of PV cells is mainly realised by LBIC measurements. This technique has been widely used for spatial characterisation of electrical properties of solar cells as well as photodiodes [198] and other semiconductor devices.

An alternative method of PV device current mapping that uses a different sampling approach is developed in this work. As it was described in the previous chapter, this method utilises the compressive sensing (CS) sampling theory, which can extract a signal from incomplete or inaccurate observations [156][14]. CS current mapping is realised by projecting a series of patterns onto the sample while measuring its current response for every pattern. The final current map is acquired via a reconstruction algorithm. The aim of this procedure is to produce an accurate current map having acquired far fewer measurements than is required 
by a point-by-point approach. Undersampling and increased signal to noise ratio have the potential to significantly increase measurement speed and simplify measurement layouts.

The proposed method is potentially a useful tool, but its performance for identifying different types of defects and features in semiconductor devices is not well defined. This will be achieved in this chapter, through a series of electrical simulations. Investigations are realised of whether the performance of this technique depends on the type of localised features in the devices to be measured. Additionally, the theoretical performance limits of the method are explored. It is shown that the technique is applicable to all types of solar cells, regardless of them being thin films or wafer based semiconductors. A demonstration of applying the method to integrated series connected devices (PV modules) is presented.

Simulated measurements are selected to provide an objective verification of the quality of the method. Electrical simulations involve modelling spatially varying solar cell characteristics. A controlled environment is provided and this enables the quantification of realistic undersampling levels for PV measurement using structured illumination and CS. A spatially resolved model based on a circuit network is used and the PV-Oriented Nodal Analysis (PVONA) toolset [199] is used to calculate the PV devices' response to various illumination patterns. Simulation results of CS current mapping measurements for the cases of c-Si samples and thin film solar cells are demonstrated. Different defect schemes are investigated, to test whether the CS current mapping method can reconstruct every kind of current response caused by non-uniformities of samples.

The potentially strongest feature of this method is also demonstrated, which is the inherent ability to scan multiple cell PV devices (modules), limiting the cell under measurement while biasing the others in the module. This means that a fully encapsulated module can be scanned at high speed, using the method's undersampling principles. The need to limit the cell under test when conducting current mapping measurements on PV modules has already been highlighted in [78]. The electrical simulations also offer a way to test some different sensing matrix groups and reconstruction algorithms.

\subsection{Compressive Sampling Using the PVONA toolset}

PV-Oriented Nodal Analysis (PVONA) is a software toolset developed in CREST for solving the spatially-resolved model (SRM) of PV devices [200]. A PV cell can be described by an SRM in the form of a discrete nonlinear circuit network as shown in Figure 4.1. In the SRM, a PV cell is modelled as an array of virtual sub-cells. Each local sub-cell corresponds to a finite 
rectangular-shaped area of the physical cell. The nonlinear behaviour of the local sub-cells, which are represented by the blocks with an arrow in Figure 4.1, is described by local diode models. The contact schemes are represented by two resistor networks, one on top and one at the bottom of the cell.
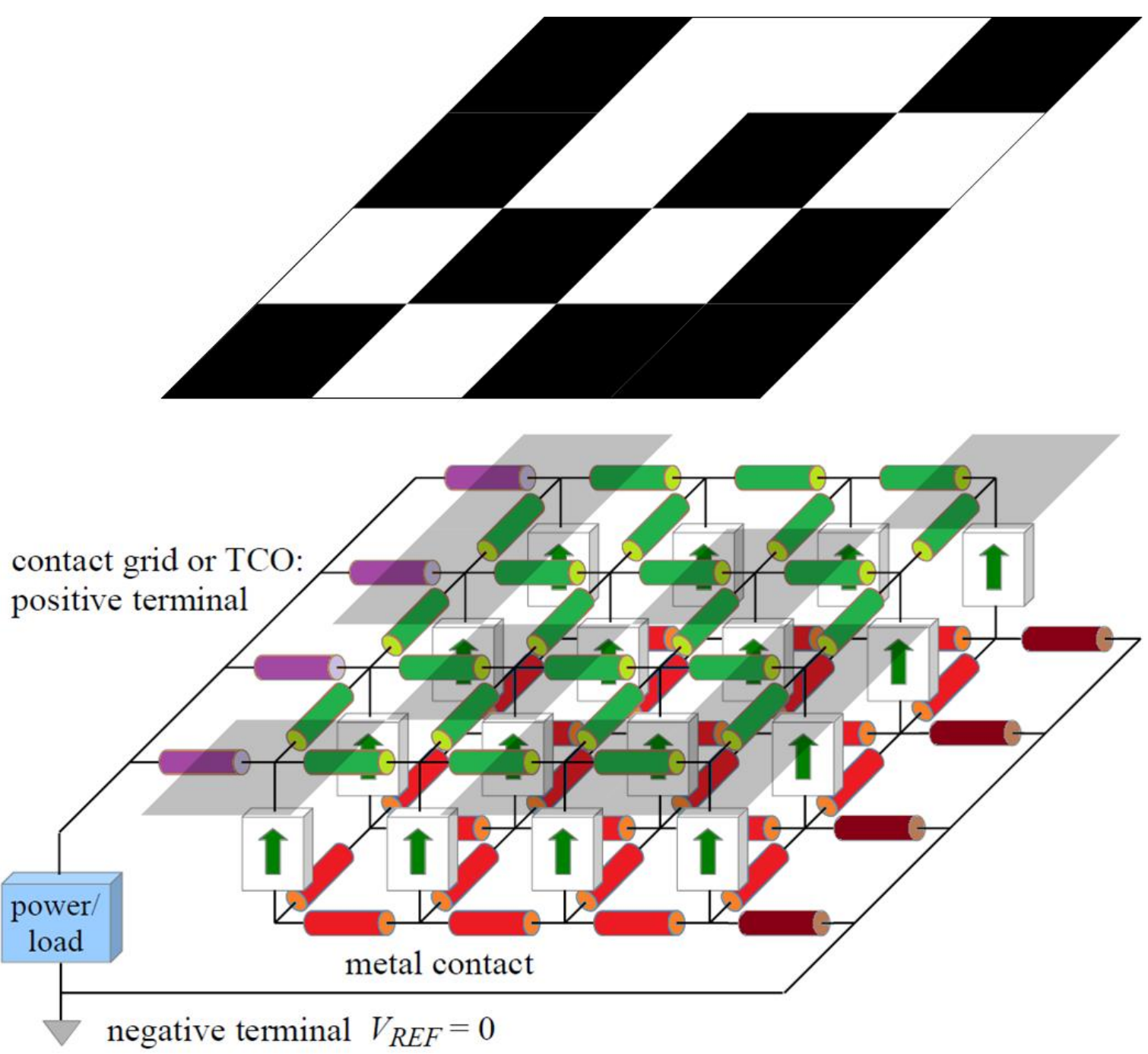

Figure 4.1. Schematic diagram of the circuit network structure of the SRM of a PV cell. A random pattern is projected on the cell for compressive sampling and the PVONA toolset solves the SRM and provides the electrical output parameters.

In the circuit of Figure 4.1, a node is a point where circuit components and branches connect. Hence, using nodal analysis the potential difference between nodes can be determined. A nodal equation system is generated by applying Kirchhoff's current law to each node. The PVONA toolset can solve the SRM efficiently by utilising an optimised parallel solver 
based on a Newton-Raphson method as described in [18]. It allows high-resolution (megapixel level) simulations of PV cells with significantly reduced computation time and memory usage. It is thus useful for investigation of the effects of inhomogeneities in PV cells and modules [199].

Compressive sampling is simulated by feeding the PVONA toolset the spatially nonuniform irradiance produced by each sensing pattern; in the sensing matrix $\Phi$ every row represents a pattern that is projected on the simulated device under test. When an illumination pattern is set for projection on the simulated devices, the PVONA toolset solves the SRM for these specific illumination conditions that the binary projection creates. The solver provides the electrical output parameters, which in this case are the global and local current and voltage values of the simulated device. The global current response to each pattern is of interest for simulating CS current mapping, as every reading populates vector $\boldsymbol{y}$, as described in the previous chapter. The complete procedure simulates CS current mapping measurements but unlike an actual experimental procedure, the results include no noise or other measurement artefacts. Consequently, the theoretical performance limits of CS current mapping can be realistically evaluated.

Two cases are examined: a crystalline silicon (c-Si) single cell and a thin film device consisting of 3 individual cells connected in series are virtually constructed for the purpose of the simulations. The simulation of the front contact layer of the c-Si sample is illustrated in Figure 4.2 (left). The sample consists of $60 \times 60$ virtual sub-cells which also defines the resolution of the final current map. A front contact layer is used to model the metal busbar and fingers and a top resistance layer is introduced to model lateral current paths. The busbar occupies 10 columns and each of the three fingers occupies one row. The resistivity of the back metal contact can be assumed to be very low and uniform, thus the back contact resistance is neglected in this study [18]. The one-diode model is used to describe local electrical properties of sub-cells, using the input parameters listed in Table 4.1. More elaborate diode models can be used but the one-diode version is used here since the aim is to assess the behaviour of the CS approach rather than precision modelling of specific PV device designs.

To assess the performance of CS current mapping measurements for different types of features, defects are introduced by changing the diode model parameters of the virtual PV cells in small, localised groups. For example, a crack or defective area can be modelled by a series of sub-cells with reduced or zero photocurrent $J_{\text {ph }}$ and a very high emitter resistance $R_{\text {memitter }}$ value, $R_{\mathbf{m}}$ being the sheet resistance. For the case of the virtual thin film device, a 3- 
cell $60 \times 60$ pixel device is configured as presented in Figure 4.2 (right). Each individual cell has a size of 20x60 with the individual cells being series interconnected. The values of the main parameters set for the thin film virtual cells are also presented in table 4.1.

\section{TABLE 4.1}

Electrical Parameters of the Simulated Samples

\begin{tabular}{lll}
\hline & $\mathrm{c}-\mathrm{Si}$ & Thin film \\
$J_{\mathrm{ph}}\left(\mathrm{A} / \mathrm{cm}^{2}\right)$ & 0.035 & 0.0135 \\
$J_{\text {sat }}\left(\mathrm{A} / \mathrm{cm}^{2}\right)$ & $5.0 \times 10^{-7}$ & $1.0 \times 10^{-10}$ \\
$\mathrm{n}$ & 1.4 & 2 \\
$R_{\text {sh }}\left(\Omega \cdot \mathrm{cm}^{2}\right)$ & $8.35 \times 10^{4}$ & $5.3 \times 10^{4}$ \\
$R_{\mathrm{s}}\left(\Omega \cdot \mathrm{cm}^{2}\right)$ & 0.05 & 0.1 \\
$R_{\text {घemitter }}(\Omega / \square)$ & 80.0 & 80.0 \\
$R_{\mathbf{m g r i d}}(\Omega / \square)$ & $1.0 \times 10^{-3}$ & 10 \\
\hline
\end{tabular}

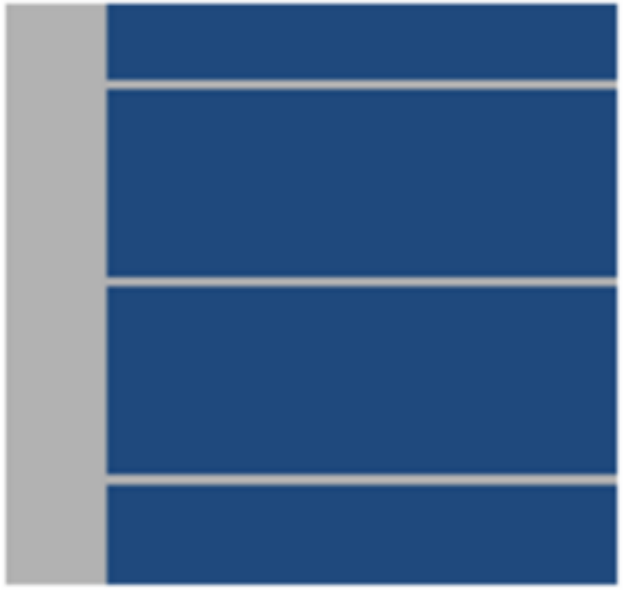

(a)

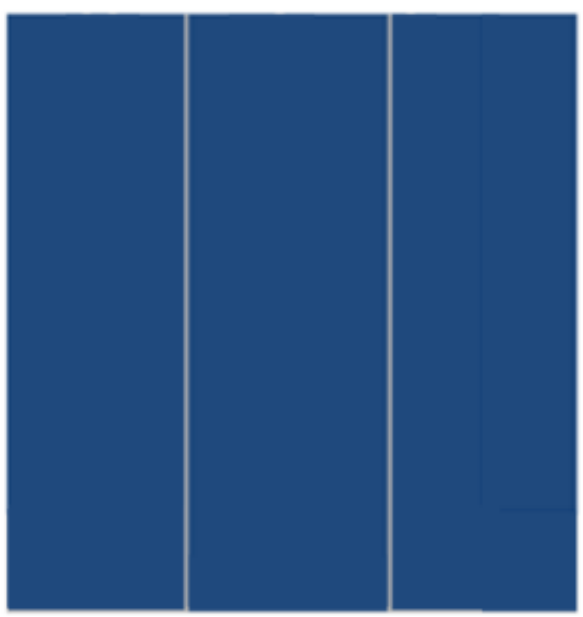

(b)

Figure 4.2. (a) The layout of the front contact layer of the c-Si virtual sample. (b) The layout of the 3 cell thin film virtual device.

The cell size is represented in arbitrary units. However, considering that a finger of a cSi PV cell front contact has a typical width of approximately $100 \mu \mathrm{m}$, the simulated c-Si sample would correspond to a $6 \mathrm{~cm}$ by $6 \mathrm{~cm}$ area of a cell. This is a reasonable measurement area for a small area optical setup, such as the one that will be presented in the next chapter. Similar 
considerations can be made for the thin film simulation. The size is arbitrary as in the previous case. Having fewer pixels for each cell $(20 \times 60)$ means that when making an approximation to actual size, an even smaller area is eventually measured. This would correspond to a small research device, where shunts or other defects would be visible. In the case of the series interconnected simulated module, the principles of compressed sensing are tested for modules, making the size totally arbitrary. This means that in actual commercial PV modules such resolution would not suffice for detection of extremely small local defects. Nevertheless, as will be demonstrated in chapter 6 , the principles used for these simulations with such a low resolution can be applied also to actual PV modules. The low resolution used here is enough to demonstrate the feasibility of CS current mapping for modules, and to test the performance limits of the method.

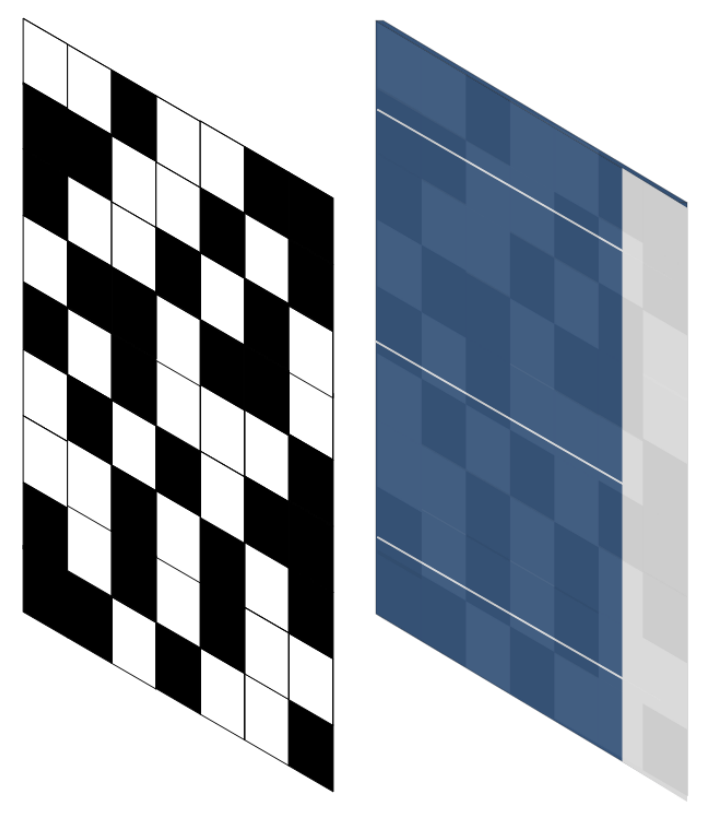

a)

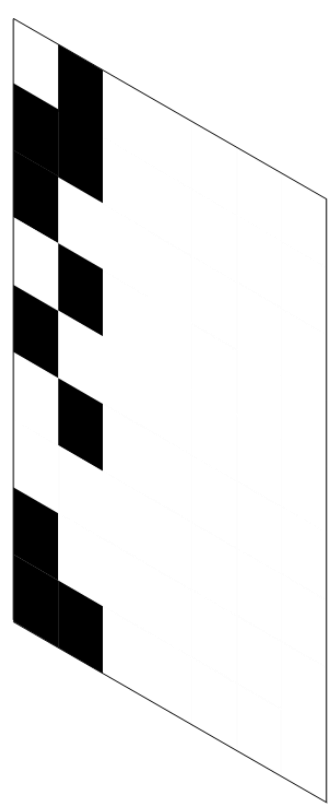

b)

Figure 4.3. The compressive sampling process for the cases investigated in this work. (a) For the c-Si case the generated random patterns are projected on the whole area of the virtual samples. (b) For the thin film 3-cell mini-module, the series of random patterns are projected onto each individual cell while uniform illumination is cast on the remaining cells. As a result, the cell under measurement is the current-limiting cell of the module.

The simulation of CS current mapping measurements is done using the irradiance configuration capabilities provided by PVONA and applying the principles described in section 
3.4. The sensing matrix $\Phi$ consists of binary matrices expressed as rows that represent the patterns to be projected onto the virtual samples. For an illuminated simulated sub-cell (pixel), the photocurrent $J_{p h}$ listed in Table 4.1 is applied. For an inactive area such as a finger or a busbar, the $J_{p h}$ is set as zero. For an underperforming sub-cell $J_{p h}$ has a reduced value. For each pattern projected, the short circuit current $\left(I_{s c}\right)$ of the overall simulated cell for the given conditions is calculated with PVONA, populating the CS measurement vector $\boldsymbol{y}$. Up to this point, simulations are realised in $\mathrm{C}++$, since the PVONA software is programmed in this environment, with the output being vector $\boldsymbol{y}$. The current map is finally reconstructed by applying a reconstruction algorithm. The $\ell_{1}$ minimisation algorithm of the $\ell_{1}$ magic toolset is used if not stated otherwise. This final step is implemented in the LabView environment, using a MatLab script to call the necessary algorithms, as described in the previous chapter.

\subsection{Simulation Results}

\subsubsection{Simulated Crystalline Silicon PV samples}

For the simulated c-Si sample, several cases of the $60 \times 60$ pixel cell of one busbar and three fingers were explored. As illustrated in Figure 4.4, six different cases were investigated; an ideal cell with no defects, 3 cells with cracks of different orientations and positions, a cell with a dead spot and a cell with a combination of these features. For the network's sub-cells (pixels) forming the defects, zero photocurrent and a much higher series resistance were set, except for the last case, in which the crack is simulated by reducing the photocurrent to $50 \%$ of the initial value, in order to achieve a more realistic case. At the points of fingers and busbars, the resistance towards neighbouring points was set close to zero, in order to simulate the high conductivity, while photocurrent was set to zero.

When applying compressive sampling, a random illumination pattern is projected on the simulated device and the PVONA toolset provides the local and global electrical output. As an example, the local current response and voltage maps generated by PVONA for a random pattern projected on the sample are presented in Figure 4.5. The simulated sample with the combination of defects is used as the input. The defects exist but are barely visible in the current response map, as the local current generation due to the projected pattern 
dominates. Only the diagonal crack appears in the local voltage map. The global current response results (i.e. the current flowing from the terminals of the device) for each pattern are of interest for simulating CS current mapping. The total current generated for each projected pattern provides the current measurements that populate vector $\boldsymbol{y}$. These are the measurements then used to reconstruct the final current map.

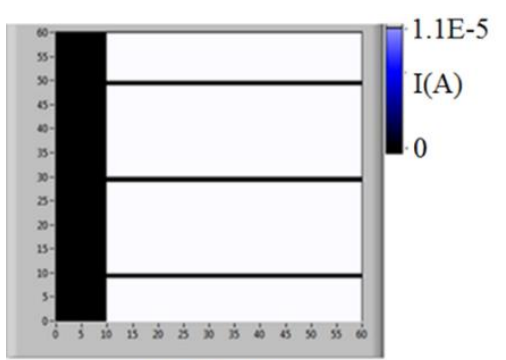

no defects

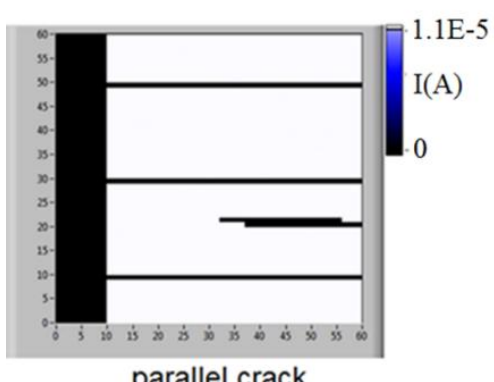

parallel crack

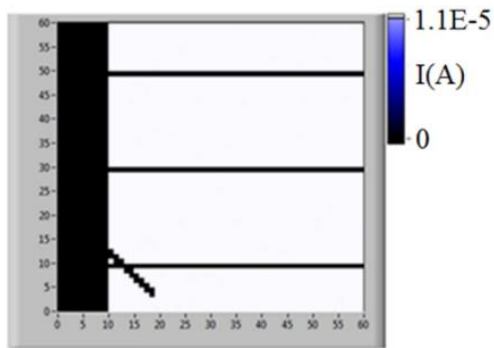

diagonal crack

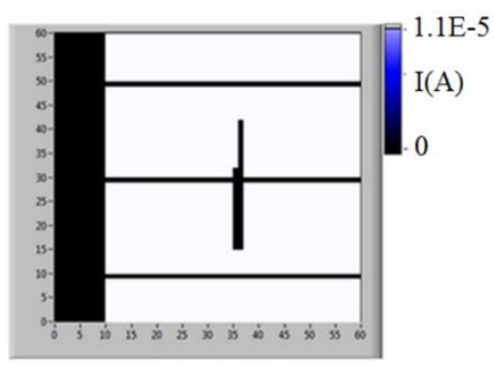

vertical track

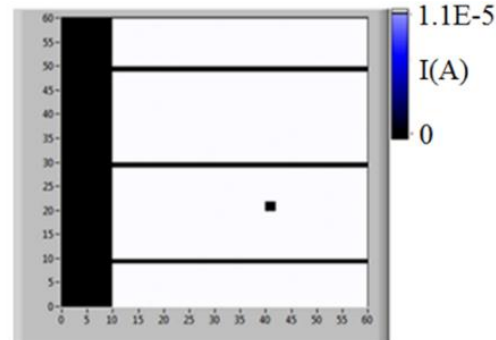

dead spot

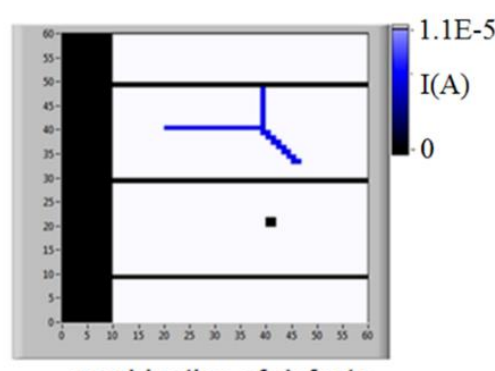

combination of defects

Figure 4.4. The actual current maps of the configured c-Si samples for the simulations of the CS current mapping method.
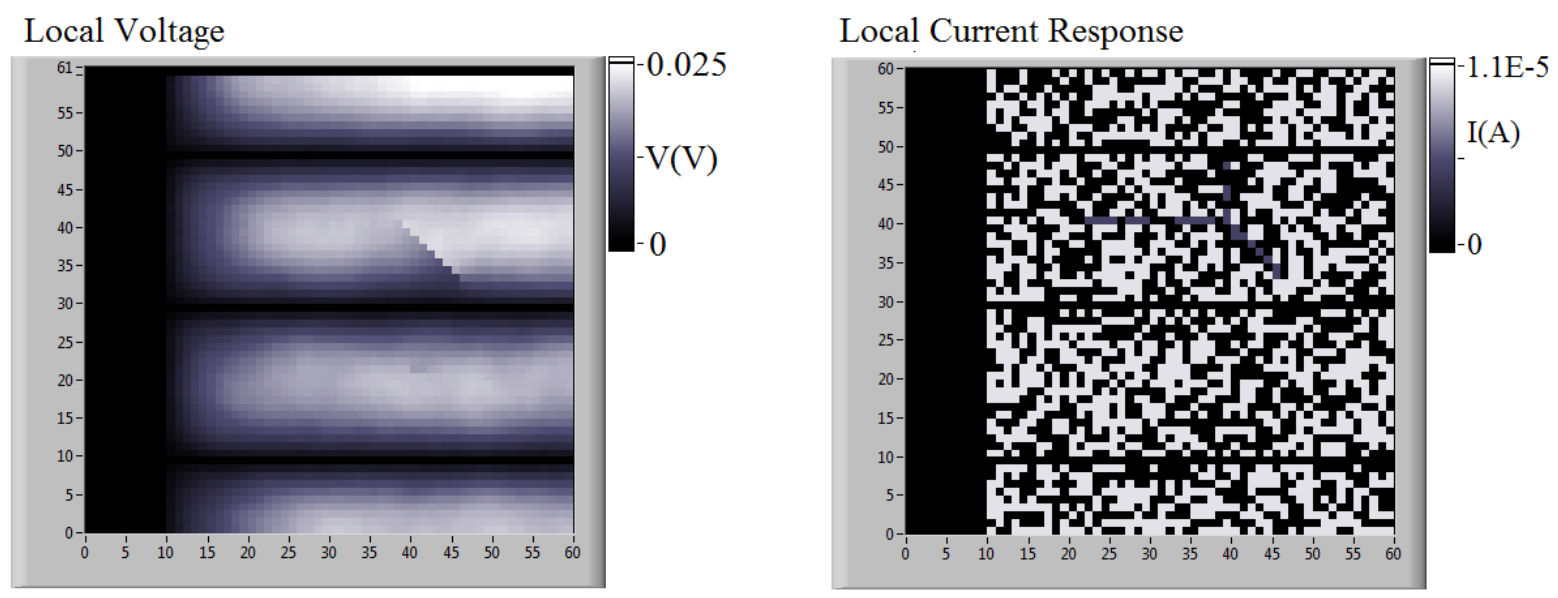

Figure 4.5. On the left, the local voltage map that PVONA generates for the simulated c-Si device with the crack and the dead spot, when projecting a random pattern on the device. On the right, the generated current response of the same simulated device when projecting a random pattern. 

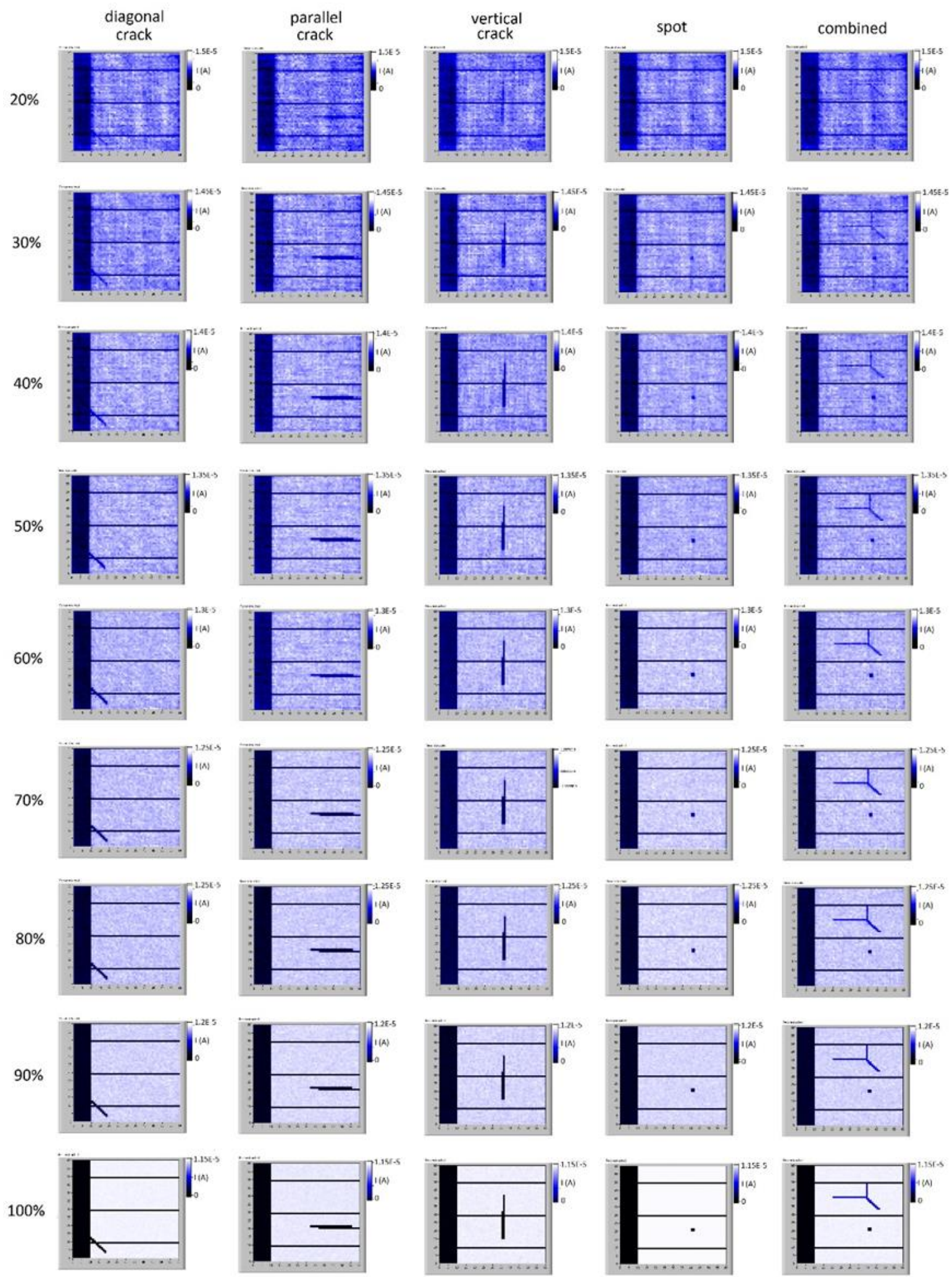

Figure 4.6 Reconstructed current maps of all the different simulated c-Si cells, with different levels of undersampling. The percentages in the left column express the ratio of number of measurements to the total number of pixels. 
The reconstructed current maps for all the simulated c-Si cells with different defects are presented in Figure 4.6. The percentages in the left column express the ratio of the number of measurements to the total number of pixels. For instance, the $40 \%$ current maps consist of 3600 pixels and were reconstructed by acquiring 1440 measurements, which mean that 1440 patterns were projected on the samples, calculating the current response for each pattern. In practice, one measurement is considered to be one pattern projected on the device to achieve a current reading. Applying a conventional raster scan, 3600 point measurements would be needed to create the 3600 pixel current map. Almost all the features of the cell are distinguishable at $30 \%$ undersampling, even if the reconstruction is still rather noisy. Adding more and more measurements the image quality increases gradually and converges to the actual current map, producing a perfect reconstruction when using $100 \%$ of the measurements (3600 measurements). In actual CS applications, the $40 \%$ or $50 \%$ current maps would already be sufficient for characterising the local current response of a device with minimal errors. The above simulation results initially confirm that CS current mapping is realistic and can in practice provide results with much fewer measurements than a traditional point by point scan would need. The reconstructed current maps give accurate quantitative results for the local current response and additionally, the method exhibits similar performance for all types of defect shapes and orientations for this simulated sample.

For a quantitative evaluation of the performance, the actual and reconstructed current maps were compared at a pixel by pixel level. Pearson's correlation coefficient and Normalized root mean squared error (NRMSE) were calculated for each level of undersampling used for reconstruction and are presented in Figure 4.7. The correlation coefficient is calculated by dividing the covariance of the real and reconstructed current map by the product of their standard deviations:

$$
\rho(\widehat{x}, \boldsymbol{x})=\frac{\operatorname{cov}(\widehat{\boldsymbol{x}}, \boldsymbol{x})}{\sigma_{\widehat{\boldsymbol{x}}} \cdot \sigma_{\boldsymbol{x}}}
$$

Where $\mathbf{x}$ is the real current map and $\hat{\mathbf{x}}$ is the reconstructed current map, in vector form.

As expected from the above results, the proposed measurement technique demonstrates quantitatively almost identical performance for all the different features investigated, as is presented in the graphs of Figure 4.7. The slightly different behaviour in the case of the samples with the vertical and parallel crack is due to the symmetry present in these samples. The DCT transform is used for providing the sparse representation, which 
means symmetrical structures can exhibit slightly different performance. An absolutely symmetrical structure would be rare in reality, but not impossible. However, even samples with highly symmetrical actual current maps will have minimal differences in convergence practically.
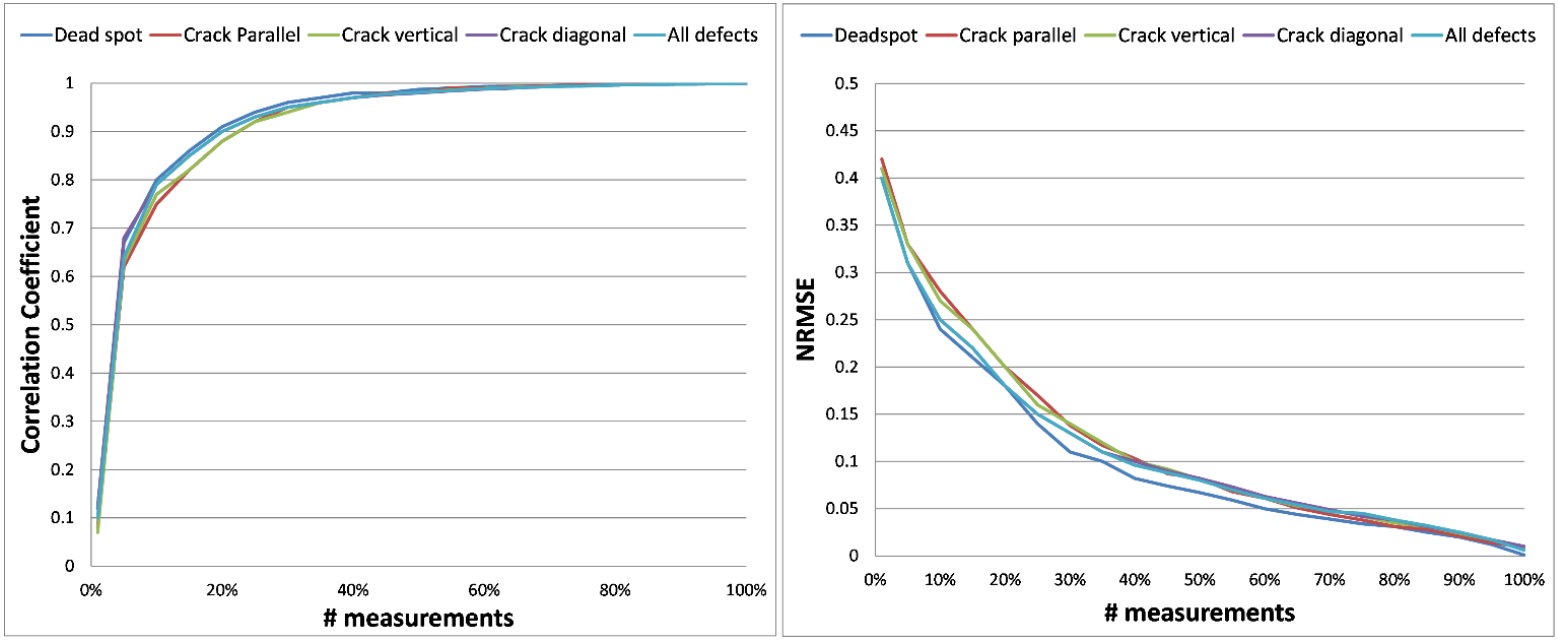

Figure 4.7. Correlation coefficient and NRMSE for the configured c-Si samples between the CS and the real current maps, as a function of number of measurements.

Current maps using more than $40 \%$ of measurements for reconstruction have Normalised Root Mean Square Error (NRMSE) below 10\% and correlation coefficient greater than 0.95. Bearing in mind the reconstruction considerations in the previous chapter, it is probable that for higher resolution samples the performance of the CS current mapping technique can have an even better performance. This means that higher degrees of undersampling can be achieved for reconstructing a reliable high resolution current map.

A reconstruction error map is presented in Figure 4.8, for the case of the combination of defects and for $50 \%$ undersampling. The error is normalised and expressed as a percentage, stating the difference between actual and reconstructed values. A maximum of $48 \%$ can be observed, at the position of the dead spot. This is due to the current value of the spot being very close to zero, making the relative error value very large. Nevertheless, the fingers and busbar areas which also have an almost zero value current exhibit a very small error, in the range of $5 \%$. This indicates that very small local defects may be more difficult to quantitatively evaluate with compressive sampling than larger structures. Nevertheless, such small defects are still detected, but in order to accurately determine their values further measurements (projected patterns) are needed. 

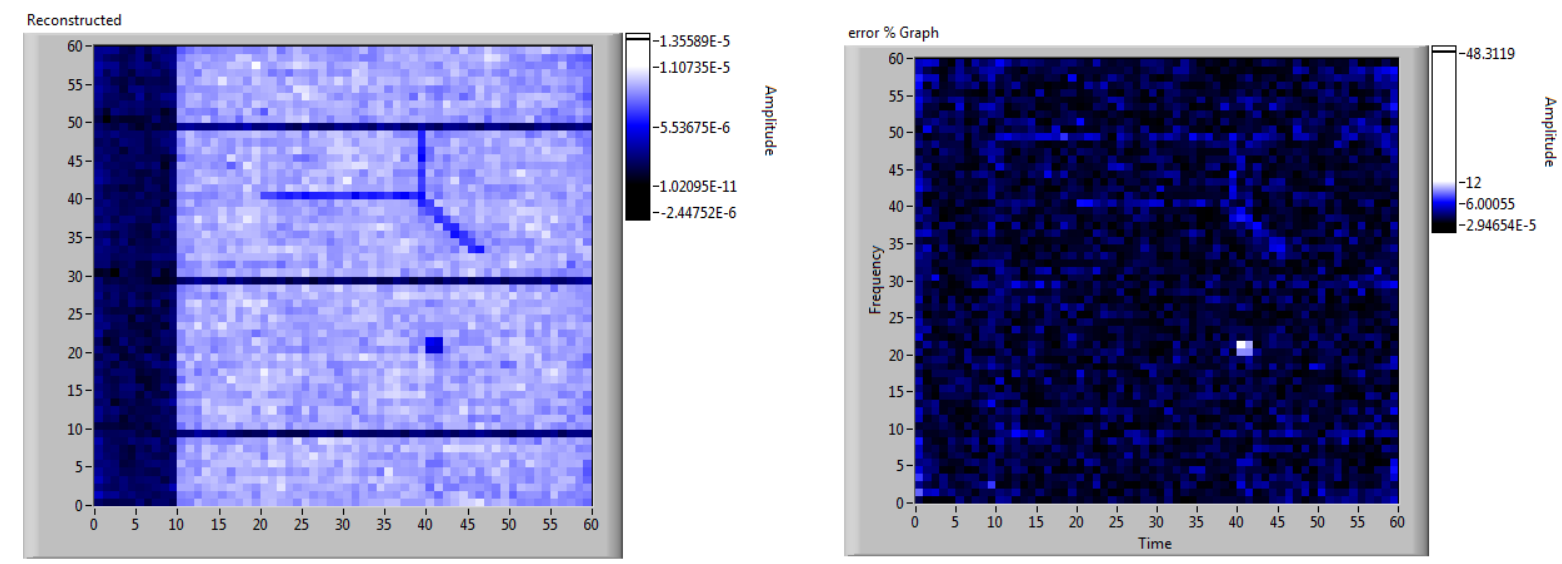

Figure 4.8. On the left, the reconstructed current map of the cell with all the defects combined, using 50\% undersampling (1800 measurements). On the right, the error map, calculated from the actual current map and the reconstructed map.

\subsubsection{Simulated Thin Film PV Devices}

For the thin film PV device case, 3 different cells were individually simulated and then combined to a single PV device. This simulates a series interconnected mini module consisting of these three cells. Three different cases of individual cell features are configured and PVONA is used to solve the SRM for each cell, with a resolution of $60 \times 20$ for each case. The first is an ideal cell without any defects, with a very uniform current response. The second case includes a cell with a large area of underperformance at one end of the cell, which in reality could represent a degraded area. The third case simulates a cell with a single small local shunt. All three cases for individual cells are presented in Figure 4.9. The parameter values of a pixel with no defects for simulating the thin film cells are presented in table 4.1.

The reconstructed current maps for these individual cells are presented in Figure 4.9, with different levels of undersampling. The simulated ideal cell is almost completely uniform, which may be a rare case in reality but it is possible to find high performance thin film solar cells with no defects. It is clear from the results that due to increased pixelation, the reconstruction process yields a rather noisy current map with the $\ell 1$ minimisation algorithm. This is an artefact that this algorithm inserts when current maps are very uniform, as will be demonstrated also in the next chapter. For this reason the OMP (Orthogonal Matching Pursuit) algorithm was used and its reconstruction results are compared with $\ell 1$ minimisation in Figure 4.9. The configured samples have low display resolution (60×20 pixels) and as a result reconstruction is not as successful as for the case of the previous $60 \times 60$ pixel cells, but it still 
gives reliable current maps for $60 \%$ of measurements and above. Higher resolution sampling would allow higher levels of undersampling, as was discussed in section 3.4.2. Nevertheless, the resolution used is enough for this specific investigation.
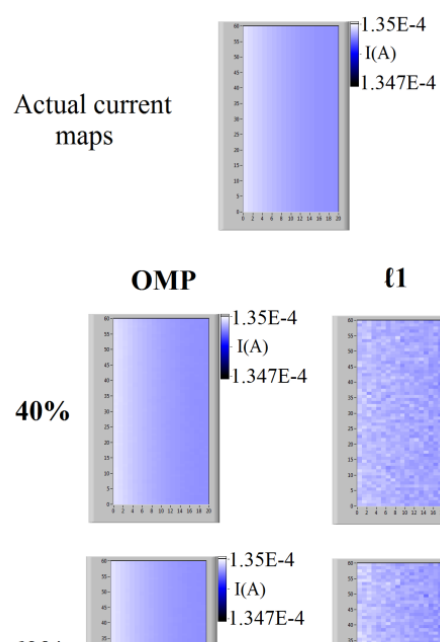

$60 \%$
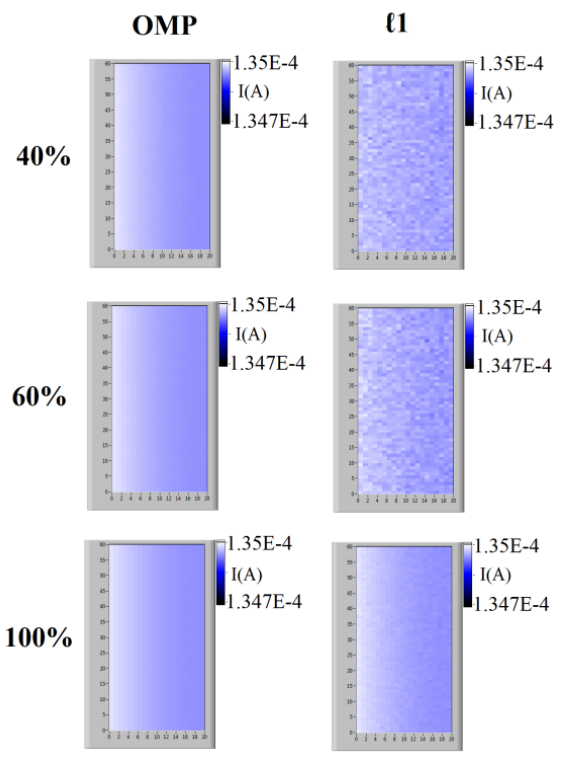
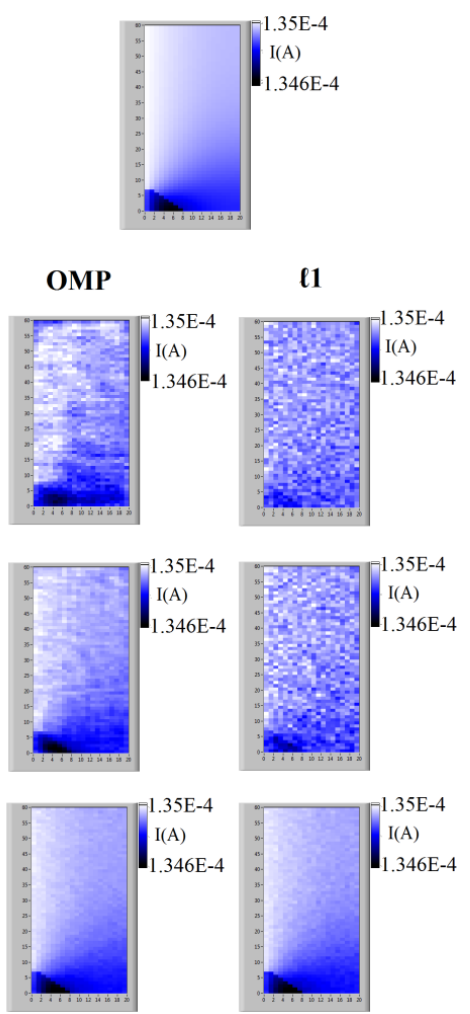

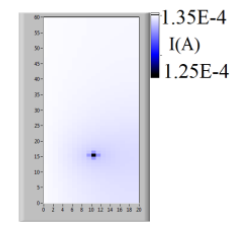

OMP
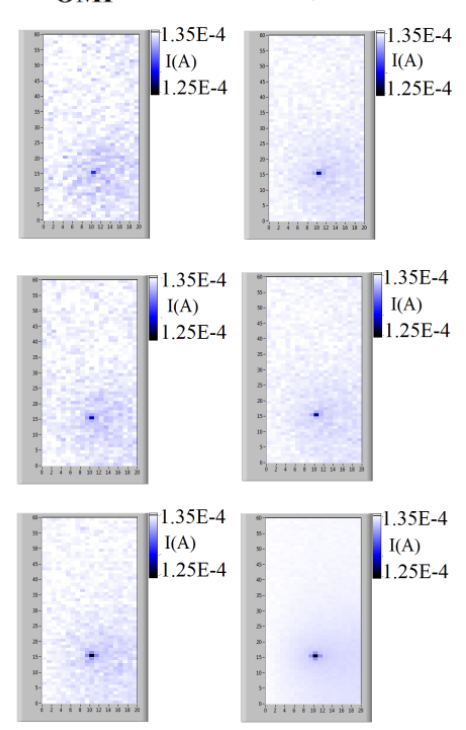

Figure 4.9. CS current maps for the different cases of thin film samples, reconstructed from different numbers of measurements, with the percentages expressing the ratio of number of measurements to the total number of pixels of the device. Reconstruction results with both $\ell 1$ minimization and OMP algorithms are presented.

For the cell with the small shunt, reconstruction with the $\ell 1$ minimisation algorithm produces a much better result compared to OMP reconstruction. For the other two cells (uniform, degraded area) OMP delivers superior results, as the actual current maps are rather uniform with no sharp structures. Between the aforementioned algorithms, the $\ell 1$ minimisation is the most suitable choice where there is no prior knowledge of the structure of the samples under test as will be discussed later, when different algorithms are compared. Each algorithm apparently has advantages and drawbacks, as some artefacts will always be inserted in the solution. The $\ell 1$ minimisation algorithm may result in pixelation when used for very smooth signals while the OMP may miss small local defects, although it works better for smooth signals. Nevertheless, there is a wide range of algorithms available in the literature 
for reconstruction after compressive sampling. The algorithms used here were selected due to their known performance and use in other CS applications, their simple implementation, their open access availability and because they belong to two different major families of reconstruction algorithm for CS.

By connecting in series all three different cells, a mini module can be simulated. CS current mapping of this PV device can be achieved by applying the necessary patterns on the cell under test while fully illuminating the others (as illustrated in Figure 4.3(b)). This is necessary to ensure that the correct current is measured every time, as has been demonstrated experimentally in [78]. The ratio of on and off pixels in the random patterns projected can be specified to ensure that the cell under measurement is always the limiting cell of the device. This feature could be crucial for devices that consist of cells with serious defects. If the current of the defective cell when fully illuminated is lower than the current of the cell under measurement, compressive sampling will not work. In this case, the current readings at the contacts of the module will not be governed by the cell under measurement but by the defective cell, producing false results. However, by reducing the number of pixels (elements of the sensing matrix) in the "on" state until the current of the measured cell is below the current of any other defective cell, such issues can be eliminated. Thus CS current mapping can work regardless of the performance of each cell in a module. The use of sensing matrices with different sparsity levels (number of pixels at the "on" state) is demonstrated in the next chapter. CS current mapping of a module with cells of different performance is demonstrated in chapter 6 . In these simulations there are no such cases, as the performance of the cells is predefined.

An overview of these simulations for the PV mini module is presented in Figure 4.10. A thin film device consisting of the three individual cells already investigated separately above is used for simulations of CS current mapping. Applying the method according to Figure 4.3(b), cell by cell compressive sampling is realised. In order to achieve higher reconstruction performance, the $\ell_{1}$ minimisation algorithm is used for reconstruction of the current map for the cell with the shunt, while the OMP algorithm is used for the other cells. The resulting current maps show that this technique can be applied to characterisation of series interconnected devices such as PV modules. This would be extremely difficult to achieve with conventional LBIC methods, as mechanical shading of the cell under measurement (to force current limiting) would be necessary in order to obtain meaningful results. 


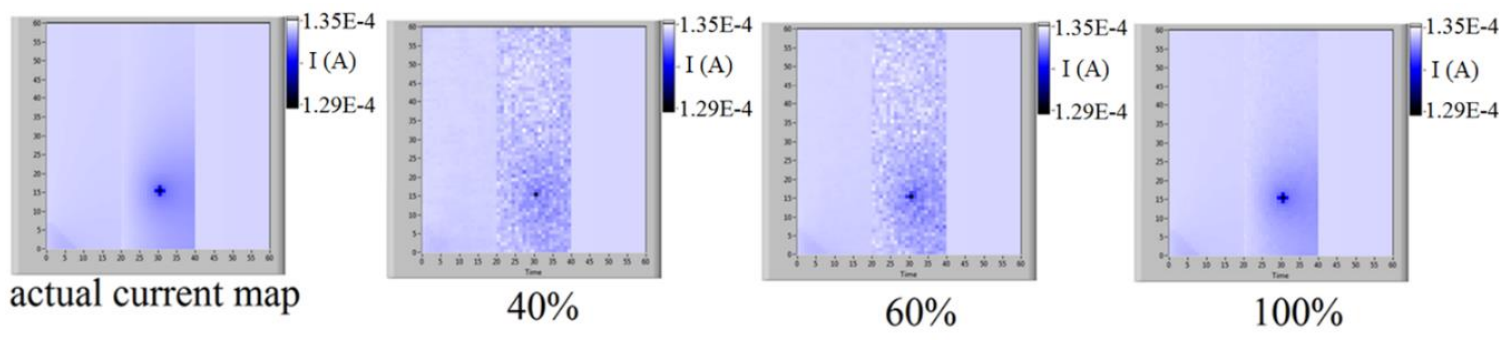

Figure 4.10. CS current maps for the 3-cell device, reconstructed from different numbers of measurements, with the percentages expressing the ratio of number of measurements to the total number of pixels of the device. The $\mathrm{e} 1$ reconstruction algorithm is used for the middle cell, while the OMP algorithm is used for the rest of the cells.

In Figure 4.11 the correlation coefficient and NRMSE are presented as a function of the number of measurements obtained, similar to the c-Si cell case. Compared to the $60 \times 60 \mathrm{c}-\mathrm{Si}$ cell, the reconstructed current map converges towards the actual current map significantly slower. However, both this graph and the reconstructed current maps in Figure 4.9 confirm that even with this low resolution the method works and results can be obtained with fewer measurements than a point by point scan, which is the purpose of this technique.
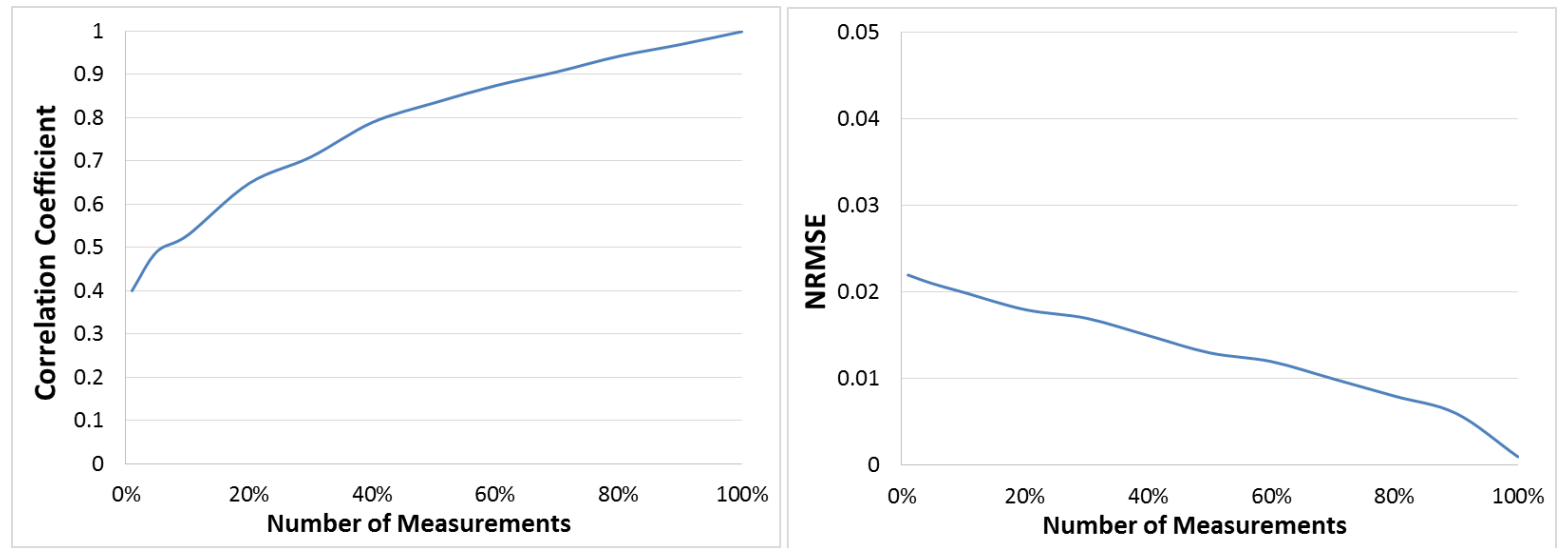

Figure 4.11. Correlation coefficient for the configured thin film device consisting of three cells, as a function of number of measurements with the CS current mapping method. 


\subsection{Comparison of Reconstruction Algorithms}

The simulation environment provides the opportunity to test several reconstruction algorithms available in the literature. This is because no measurement noise or other issues encountered in experimental work can influence results. Hence, reconstruction artefacts and convergence speed mainly depend on the reconstruction algorithm used. Several algorithms are investigated in order to select the most appropriate to be used throughout this work. The algorithms included are open access implementations in MatLab and were not developed in this work, neither were any changes applied to them. The algorithms compared are $\ell_{1}$ norm minimisation, Total Variation (TV) norm minimisation, Spectral Projected-Gradient $\ell_{1}$ (SPGL1), Dantzig selector algorithm for $\ell 1$, Orthogonal Matching Pursuit (OMP) and Compressive Sampling Matching Pursuit (CoSaMP). The first four are norm minimisation algorithms and they are all variations of the $\ell_{1}$ norm minimisation algorithm, apart from the TV which uses a different norm. The OMP and CoSaMP are greedy algorithms. A brief summary of each of these algorithms is given in the Appendix of this work. The reconstruction accuracy and speed of these algorithms are evaluated through these simulations and are presented in this section.

The accuracy of the reconstruction is investigated by calculating the NRMSE and the correlation coefficient of reconstructed current maps, when compared with the actual current map of the cell. The simulated c-Si cell with the combination of defects is used in this instance. The results are presented in Figure 4.12 for different levels of undersampling (number of measurements). All algorithms converge to the real current map as the number of acquired measurements increases. All of them also exhibit similar trends but different performance.
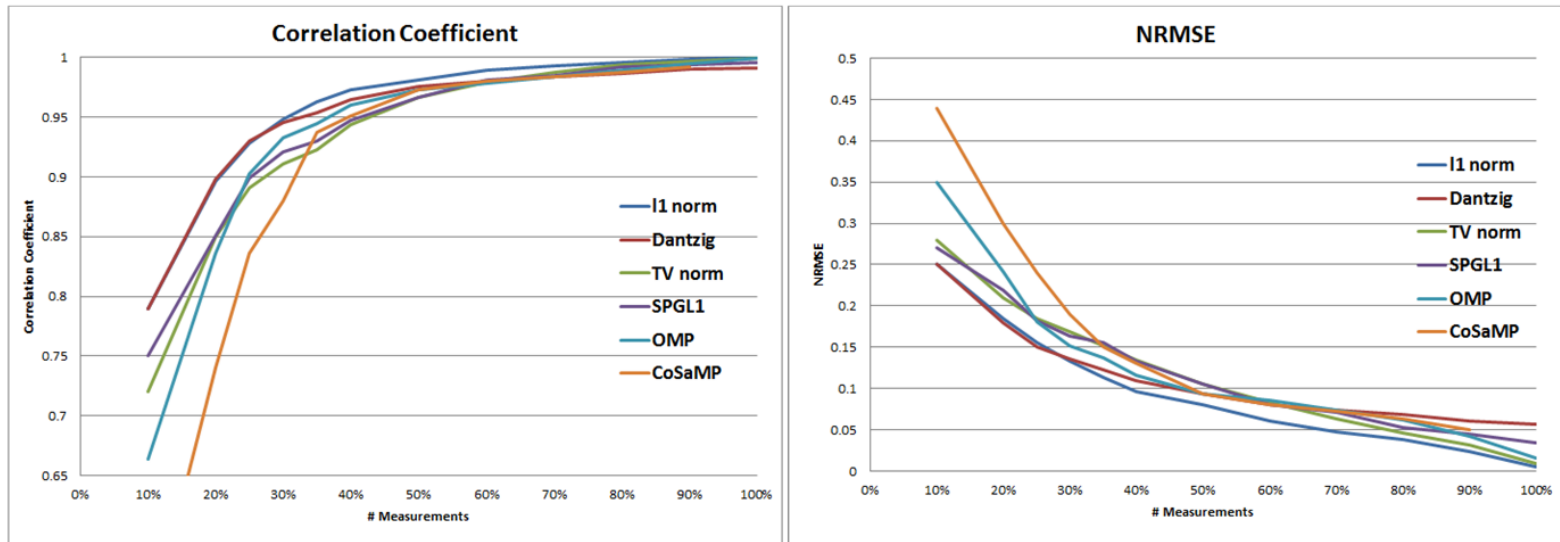

Figure 4.12. On the left, the NRMSE for the reconstructed current maps using the different algorithms. On the right, the correlation coefficient between the reconstructed current maps and the actual current map, using different algorithms. 
The results indicate that among the algorithms used, the $\ell 1$ minimisation algorithm has clearly the best performance for reconstruction for this specific CS application. The difference may be small, but especially in the range of interest, above $30 \%$ of measurements acquired, the $\ell 1$ minimisation algorithm consistently outperforms the others. Reconstructed current maps with 50\% undersampling using all these algorithms are presented in Figure 4.13. There are visually very small differences, but it can be observed that the $\ell 1$ exhibits slightly better performance than the others in the comparison. The defects inserted in the simulated cell (crack, spot) are slightly sharper in the case of $\ell 1$ reconstruction, although the differences are marginal.

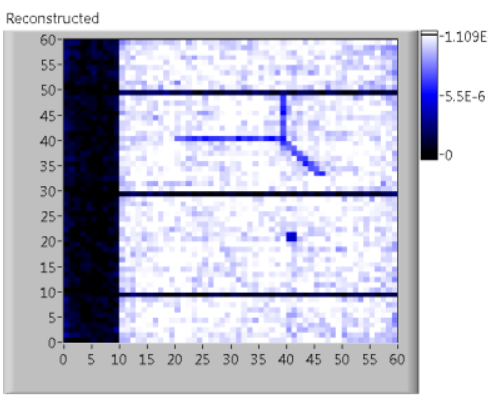

11

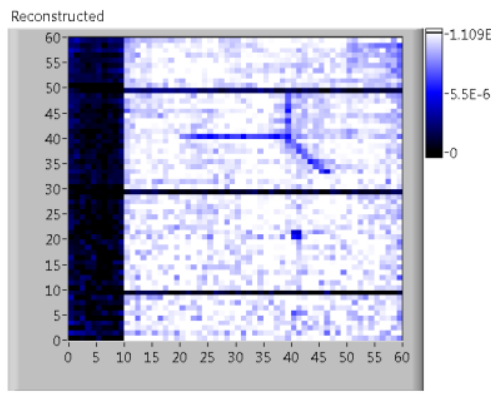

TV

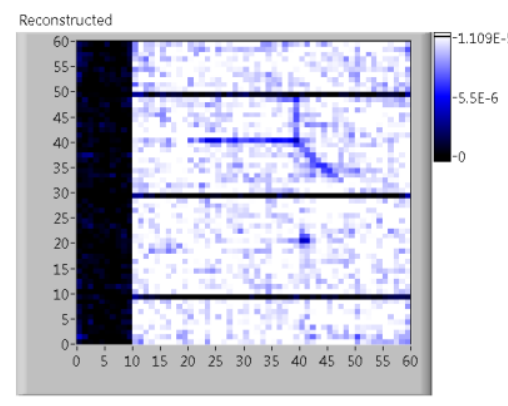

OMP

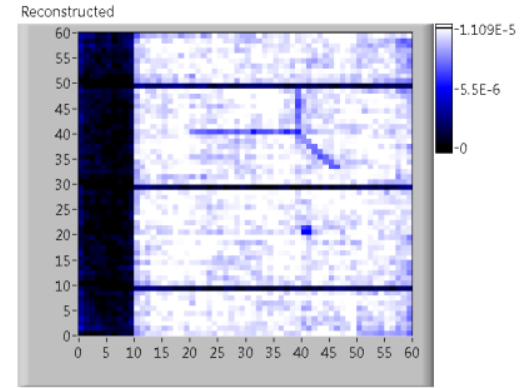

I1 Dantzig

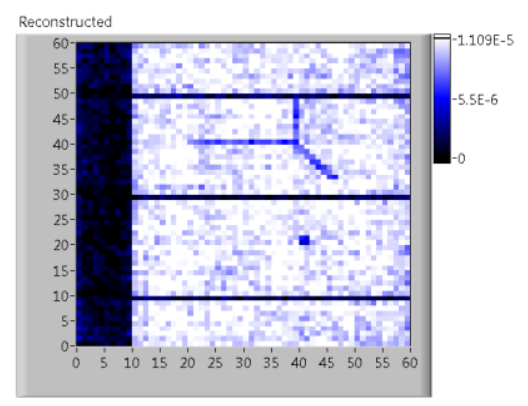

SPGL1

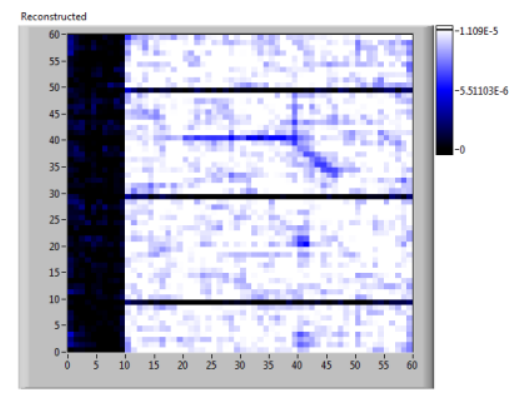

CoSaMP

Figure 4.13. Current map comparison of the different reconstruction algorithms included in this work, using 50\% undersampling (50\% acquired measurements).

A second property of the algorithms that is studied is the processing time needed for reconstructing a current map, depending on the levels of undersampling. This will help to select the best performing algorithm not only in terms of accuracy but also in terms of reconstruction speed. An Intel i5, 3.4 GHz, 28 GB of RAM computer is used. In Figure 4.14, reconstruction time is presented in seconds, as a function of number of measurements, for all the investigated algorithms. The graph clearly indicates that the $\ell 1$ and SPGL1 algorithms are the fastest and they exhibit almost identical speed, reconstruction is achieved within 
several seconds. The OMP algorithm also has similar performance up to $60 \%$ of measurements acquired, when reconstruction time starts increasing significantly. The rest of the algorithms are slightly slower, but still reconstruction is always achieved in less than a minute, except for the case of CoSaMP. In this latter case, reconstruction time is much larger compared to the other algorithms, in the range of minutes. It is also apparent from the graph that the two types of algorithms used here have different behaviour; the OMP and CoSaMP (Greedy algorithms) exhibit similar trends, although in different magnitude ranges. The same stands for the norm minimisation algorithms.

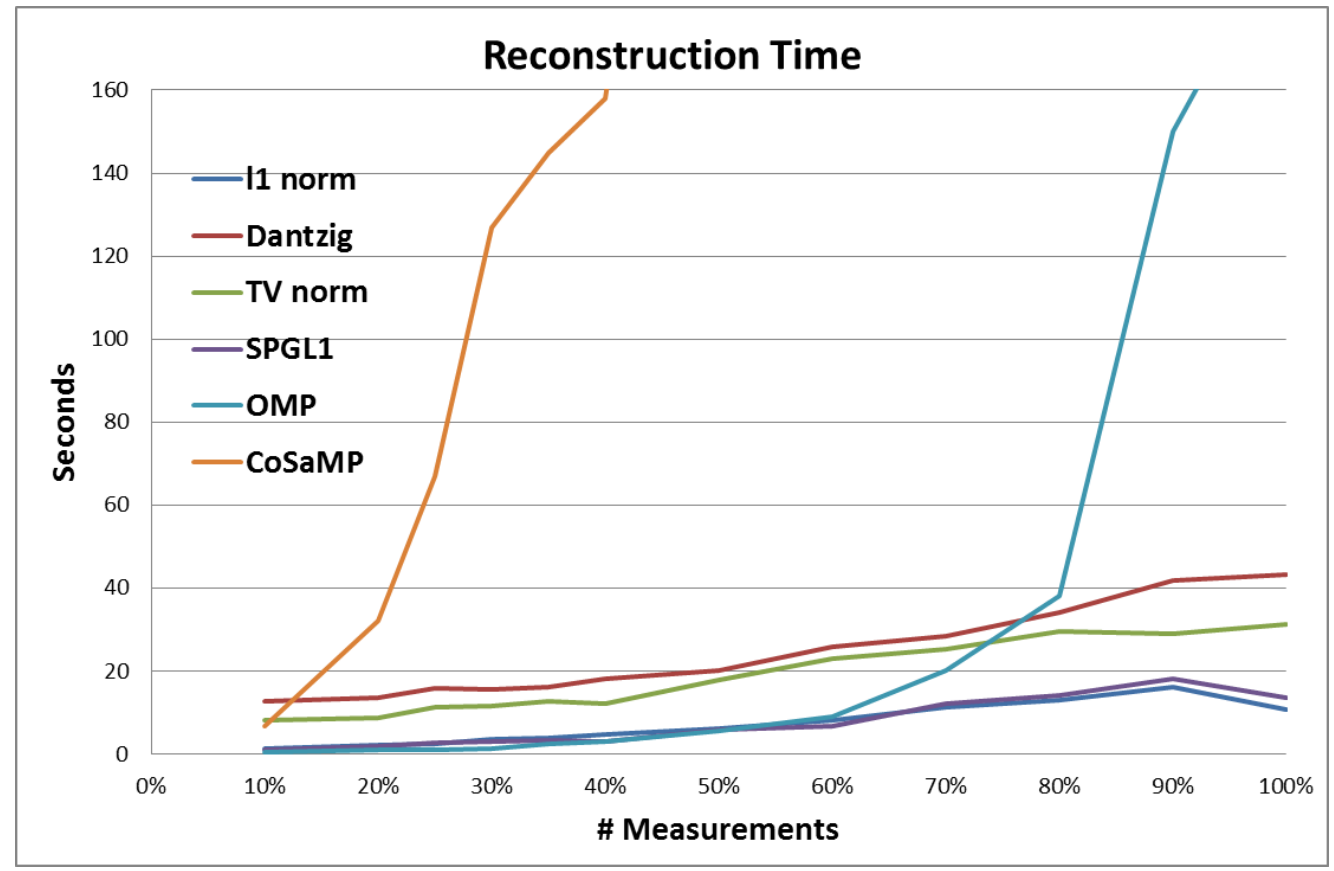

Figure 4.14. Reconstruction time in seconds that each algorithm needs for the case of the c-Si with the combination of defects.

The results presented in this section clearly indicate that the most suitable reconstruction algorithm for this application among those investigated is the $l 1$ minimisation algorithm. There are a vast number of reconstruction algorithms available in the literature, most of them having open access usage rights. Nevertheless, it is outside the scope of this work to benchmark all the available algorithms in order to define the best for this CS application. Although some effort has been spent for a minimal comparison of the most established algorithms, it is possible that better performing algorithms exist but have not been considered in this work for this application. In addition, it is very likely that better 
algorithms will be developed in the future, utilising advances in mathematics and processing power of computers.

It is expected that increasing resolution will result in increasing reconstruction time. This consideration was investigated in section 3.4.2 of the previous chapter. In this case of CS current mapping simulations, $60 \times 60$ pixel maps were used. In the following chapters in which experimental implementations are presented, the selected resolution is in most cases $100 \mathrm{x}$ 100 pixel maps. Reconstruction time is slightly increased, but is always under a minute for such a resolution level.

\subsection{Sensing matrix comparison}

In this section the sampling end of CS current mapping is investigated. Two different approaches for producing the sensing matrices to be used for compressive sampling are explored. The first is by generating random binary matrices based on several different distributions. The second is using structured binary matrices, in our case, scrambled block Hadamard (SBH) binary matrices, as it has been reported that they can be used for compressive sampling [176]. The $\ell 1$ norm minimisation algorithm is used in all cases for the reconstruction of the current maps. The results for the correlation coefficient and NRMSE for the reconstructed current maps as a function of number of measurements acquired are presented in Figure 4.15. For each point, an average of 100 runs was calculated, generating a new sensing matrix in each run for compressive sampling. For the randomly distributed matrices the generators used were Gaussian noise, Bernoulli noise, Poisson noise and uniform noise. This means that the generation of random ones and zeroes in the sensing matrix was based on different distributions. The aim of exploring different distributions is to study if they have any influence on the performance of the method. Zero is set as the mean value of all distributions, with negative values generating zeroes and positive values ones, which populate the sensing matrix in every case. This means that each element has the same probability to be one or zero.

The graphs indicate that random matrices have better performance in the range below $30 \%$ of measurements than SBH. However, in the range of interest (above $40 \%$ ) there is no difference in the performance of the different approaches so the choice of one or the other has no impact on reconstruction. Similarly, the different types of random binary matrices 
themselves have almost an identical performance. In this work uniformly distributed random binary matrices will be used for sampling, as they are very easy to implement. There are several approaches of creating sensing matrices for CS in the literature of either structured or random types and it is possible that a better approach may exist. Nevertheless, random sensing matrices are favoured here since they are guaranteed to meet the necessary incoherence criterion (chapter 3).
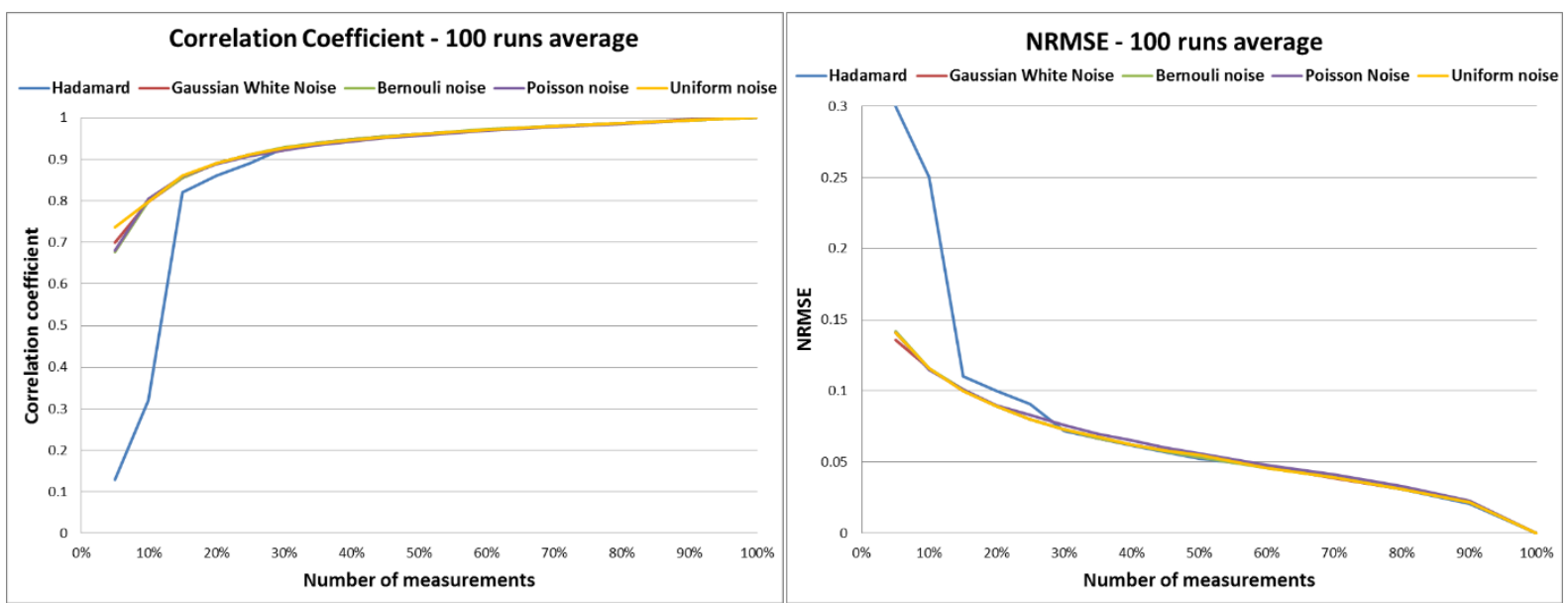

Figure 4.15 Correlation coefficient and NRMSE of reconstructed current maps in comparison to the real current map, using different sensing matrices.

\subsection{Signal levels, dynamic range and noise considerations}

As was presented above for the case of the simulated c-Si cell, the maximum current value of a pixel-point of a virtual cell is approximately $11 \mu \mathrm{A}$, which in real applications would represent an area corresponding approximately to a $100 \mu \mathrm{m}$ diameter spot, as discussed earlier. This is the signal level that a conventional LBIC system would have to accurately measure to produce the current map. In addition, a higher optical resolution scan would result in even smaller current values, in the range of nA. In Figure 4.16, the values of the elements of the CS measurement vector y for the case of the simulated c-Si cell are presented. All the values are in the range $15.1 \mathrm{~mA}$ to $16.1 \mathrm{~mA}$, which means that the current signal is enhanced by at least three orders of magnitude. This is an important feature that can be highly 
advantageous in cases where the signal level of individual pixel-points is very weak to measure with a point by point process, as will be demonstrated in the next chapter.

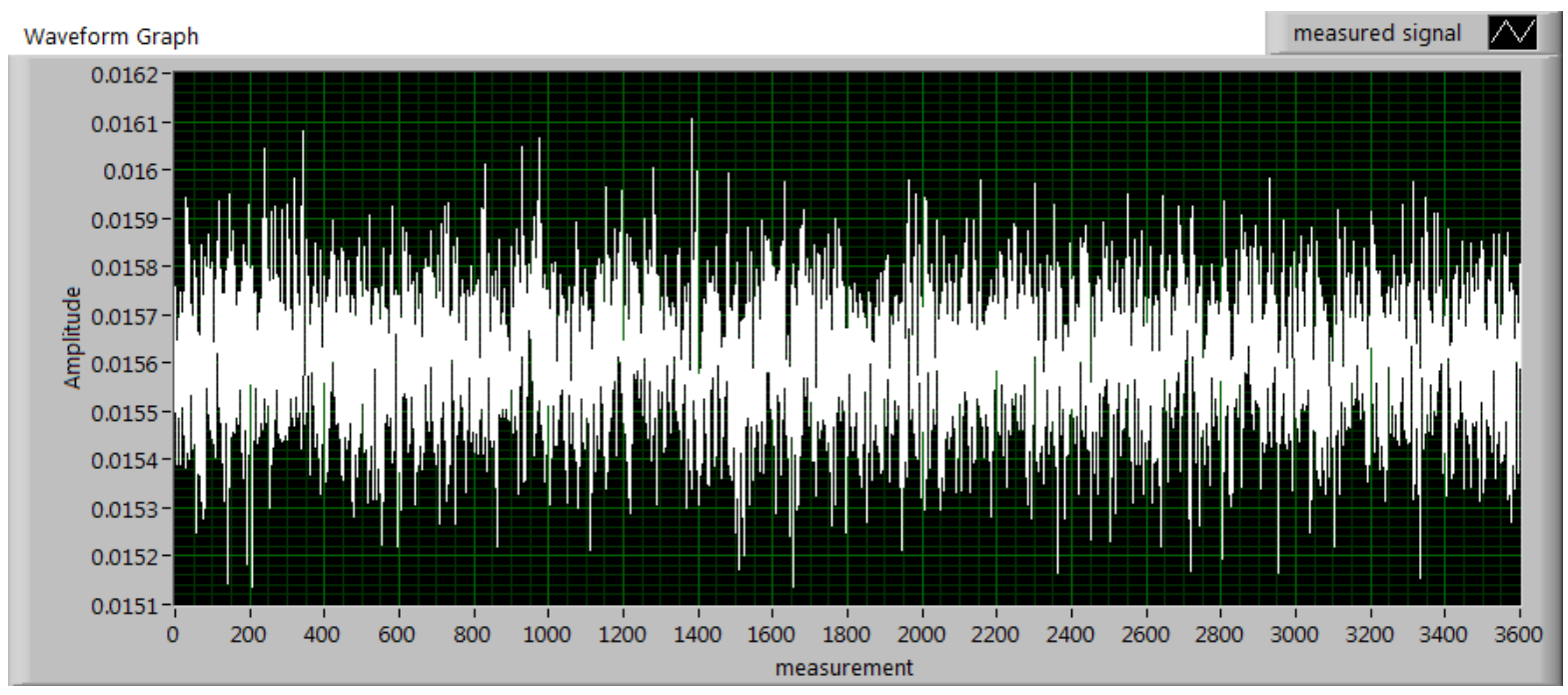

Figure 4.16. The values of all the elements of the measurement vector $y$ resulting from CS current mapping simulations. All the values are within a very small range. Each element (measurement) is the global current response of the simulated cell when projecting a single pattern.

Another significant feature that can be observed in Figure 4.16 is the fact that all values are within a very narrow range $(15.1 \mathrm{~mA}$ to $16.1 \mathrm{~mA})$. This means that when acquiring measurements, the minimum and maximum measurement reading range can be set easily in a way that provides a very high dynamic range for the sampling procedure, which can increase accuracy of measurements. This feature of compressive sampling has already been demonstrated in the literature [201]. Additionally, problematic measurements will appear as outliers and can be excluded easily from the reconstruction process. Although the signal levels are greatly enhanced with compressive sampling, actual measurements will be contaminated with noise, which slightly influences the reconstruction process.

In order to evaluate the influence of noise on the reconstruction process, the simulated compressive sampling measurements (vector y) are contaminated with several levels of uniform white noise. The levels of noise selected are randomly distributed in the range of $0.1 \%, 0.2 \%$ and $0.5 \%$ of the signal value. The reconstruction results are presented in Figure 4.17. Apart from the $\ell 1$ minimisation reconstruction algorithm, the implementation of $\ell 1$ minimisation reconstruction including measurement noise is used ( $\ell 1$ denoise). This algorithm has slightly more relaxed constraints to include measurement noise, as described 
in the Appendix. It is clear from the graph that background noise has an impact on the reconstruction process, while the use of the $\ell 1$ denoise algorithm does not provide better results in any case. Reconstruction for $100 \%$ sampling fails completely in all instances and this is also confirmed in the next chapter where actual experimental results are used.

In practice, as signal levels are significantly enhanced by using compressive sampling, the measurement noise levels are kept very small, in most cases below $0.1 \%$ of the signal. As will be demonstrated in the following chapters, there are occasions where compressive sampling can provide current maps where point by point scans fail completely due to weak signals or high background noise levels.

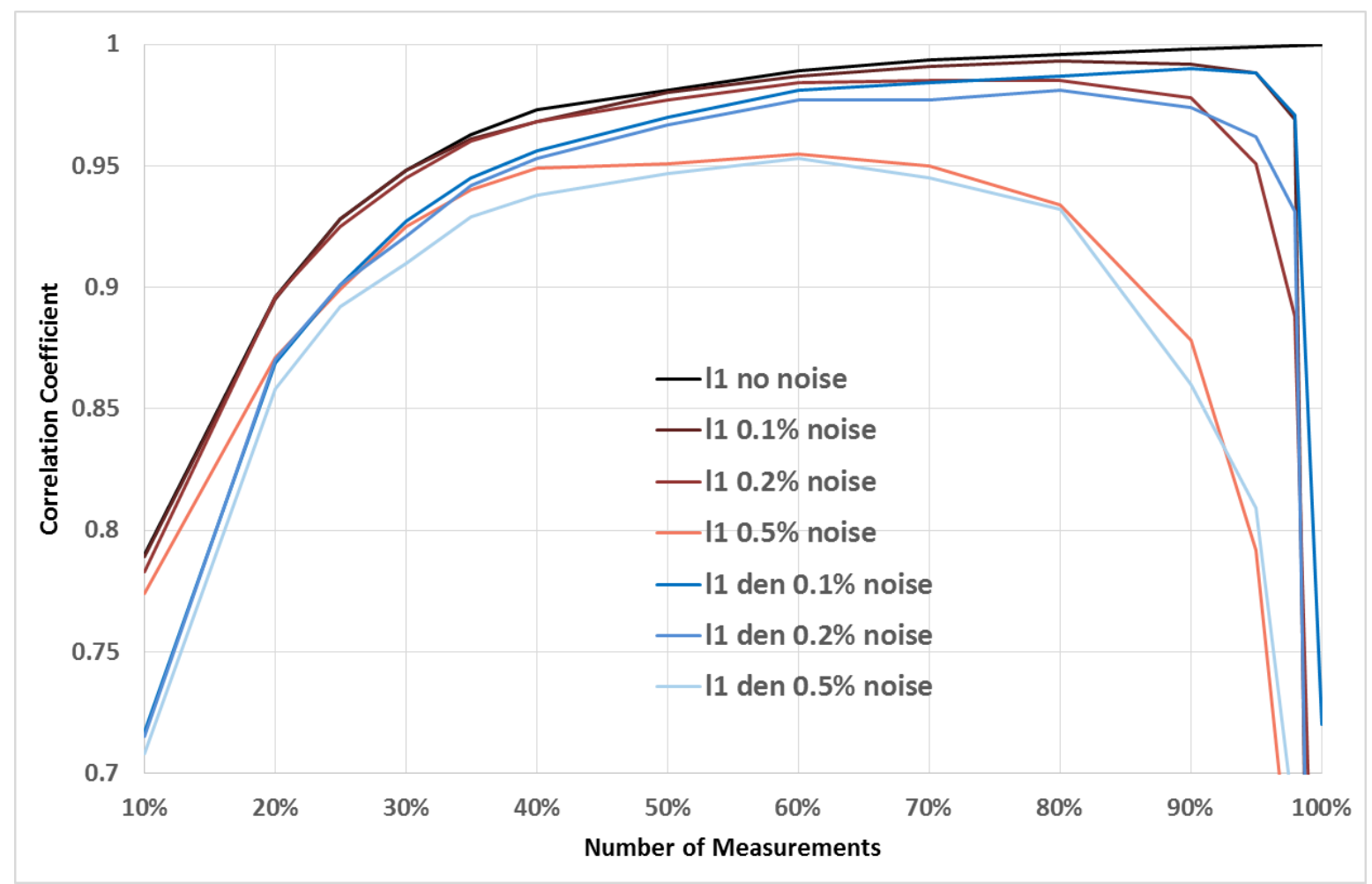

Figure 4.17. Correlation coefficient as a function of number of measurements, between the reconstructed and the real current map, with increasing levels of measurement noise, using two different implementations of the $\mathrm{\ell} 1$ norm algorithm. The random noise is expressed as a percent of the signal value. 


\subsection{Conclusions}

Simulations of CS sensing current mapping were implemented in this chapter, to theoretically confirm the feasibility of the method and evaluate its performance. The spatially resolved electrical simulations were realised using the PVONA toolset for solving the SRM of solar cells. Both simulated $\mathrm{c}-\mathrm{Si}$ and thin film devices were investigated. A realistic undersampling level demonstrated is approximately $40 \%$ of the measurements a standard LBIC system would require. This shows that this method can be at least one order of magnitude faster than the LBIC method, saving a significant amount of time. The performance of CS current mapping is the same, regardless of the features a sample may contain. Finally, results demonstrate that CS current mapping can be theoretically applied to any type of photovoltaic device. A significant feature of CS current mapping that is demonstrated through these simulations is the ability of the method to provide current maps of PV modules. This will also be experimentally verified in chapter 6 [cross ref]. Current mapping of PV modules with series interconnected cells is almost impossible to achieve with conventional raster scanning LBIC systems.

Since the simulations provide an environment where measurement noise is absent, the performance of different reconstruction algorithms and sensing matrices was explored. Among the several algorithms explored, the $\ell 1$ minimisation exhibited the best performance and is selected for used throughout this work. For the sampling procedure, random binary sensing matrices are preferred to structured matrices as they are easy to implement and their performance is slightly better.

A vast number of reconstruction algorithms and sensing matrices exist in the literature and can be tested for this application. However, a benchmarking of all the algorithms and sensing matrices available is outside the scope of this work. As the role of the reconstruction algorithm is essential, future work can concentrate on more investigations for the determination of the most suitable algorithms for this specific application of compressive sampling. The most significant characteristics of a reconstruction algorithm are speed and ability to solve large-scale problems, as well as tolerance to measurement noise. This can enable the application of CS current mapping for very fast acquisition of high resolution current maps of solar cells and other semiconductor devices.

A significant advantage of compressive sampling is that the patterns that are projected on the sample illuminate a large area instead of just a small point, which is the case in 
conventional raster scanning LBIC systems. As a result, the signal levels can be greatly enhanced by at least three orders of magnitude, depending on the sensing matrices used. This can be very practical when samples have a very weak signal, or when the background noise levels are too high to implement a point by point scan. Nevertheless, it will become clear in the next chapter that in CS current mapping there is a compromise between measurement accuracy and amplification of the signal. The $\ell 1$ minimisation algorithm performs well even in the presence of noise, although reconstruction for $100 \%$ sampling always fails in this case. On the other hand, the sampling range of interest when CS is used is within $40 \%$ to $70 \%$ of measurements, thus the reconstruction problems in the presence of noise over $90 \%$ are irrelevant in this application.

The insights of this chapter will prove useful for the experimental implementation presented in the next chapter. The choices of sensing matrices and algorithms made here will also be adopted for the experimental cases. Compressive sampling is expected to significantly enhance the current signal and measurement results are expected to be within a small range of values. Reliable reconstructed current maps are expected for measurement acquisition of more than $40 \%$. Noise will always be present in the experimental implementation of such measurements, thus reconstruction using $100 \%$ compressive sampled data is expected to fail. Whether these expectations are met or not is discussed in the following chapters, where experimental implementations of CS current mapping for PV devices are presented. 


\section{Chapter 5}

\section{Small Area Compressed Sensing Current Mapping System}

\subsection{Overview}

The main advantage of LBIC mapping is that the same principles that generate current in real applications of solar cells and modules are applied. On the other hand, a well-known drawback of this measurement technique is that it lacks speed. The small laser spot, usually of a diameter in the micrometre scale, needs to scan the entire area of the cell for a complete current map. This means the smaller the laser spot size or the bigger the sample under test, the lengthier the measurements. Thus, measuring entire PV modules or full wafer cells is usually very time consuming as the production of maps at useful spatial resolutions requires large numbers of individual point measurements. In addition, LBIC systems are usually complicated, high precision systems that also require lock-in methods for accurately measuring the very low current response of the device under test to the small area illumination.

The aim of this chapter is to demonstrate a hardware implementation of an alternative approach for current mapping of PV devices, which has the potential to reduce measurement time and simplify experimental layouts. The main aspects of the proposed method are the utilisation of a DMD kit and the application of compressive sampling for acquiring 
measurements. As discussed in chapter 3, according to the CS theory one can reconstruct a signal from highly incomplete or inaccurate information. Compressed sensing provides a way of acquiring a compressed version of the original signal by taking only a small number of linear measurements and then reconstructing an almost exact approximation of this signal. More precisely, using compressed imaging, an $\mathrm{N}$ pixel image can be reconstructed from $\mathrm{M}<<\mathrm{N}$ observations. Instead of applying a raster scan, a series of patterns are projected on the PV sample using a DMD, acquiring fewer measurements $(\mathrm{M})$ than the pixels of the final current map (N). The final reconstruction of the current map is achieved by means of an optimisation algorithm, exploiting the compressibility (sparse representation after a transform) of the measured signal.

In the previous chapter, spatially resolved electrical simulations showed that CS current mapping is theoretically possible and can actually decrease measurement acquisition time to half of what a raster scan would need. Additionally, it can be applied to both crystalline silicon and thin film devices and the performance of the method is independent of the specific spatial features or type of defect a sample possesses. The next step of this work aims on confirming experimentally the simulation results of the previous chapter.

In this chapter an analytical experimental implementation of a small area CS current mapping system for PV devices is described. The implementation was realised in several stages and it was an ongoing development process until the final experimental implementation. The layouts of two different stages are presented in this chapter; the first design and implementation related to this work, including measurement results; the final optimised version of the CS current mapping measurement system and final measurement results. All the experimental work described in this chapter took place at NPL where the optical setup was built. 


\subsection{Initial CS Current Mapping System}

\subsubsection{Optical system design}

The experimental setup is presented in Figure 2.1. Two laser sources are available, a 40 $\mathrm{mW}$ laser at $658 \mathrm{~nm}$ wavelength and a $100 \mathrm{~mW}$ laser at $785 \mathrm{~nm}$. A single mode fibre delivers the light to the optical system in both cases. The beam is expanded and collimated in order to overfill the micro-mirror array (DMD), which is a V-7000 module. It has a size of approximately $1.4 \mathrm{~cm} \times 1.0 \mathrm{~cm}$ and consists of a 1024 by 768 array of square micro-mirrors, each of $13.7 \mu \mathrm{m} \times 13.7 \mu \mathrm{m}$ size. The DMD is the most significant part of this system, as it plays the role of the pattern generator. The output of the single mode fibre creates a beam with a Gaussian profile. Overfilling the DMD with the central part of the beam results in a quasi-tophat beam profile, which means it is not perfectly uniform but sufficient for this series of experiments. The maximum difference in intensity due to this non-uniformity is below $10 \%$.

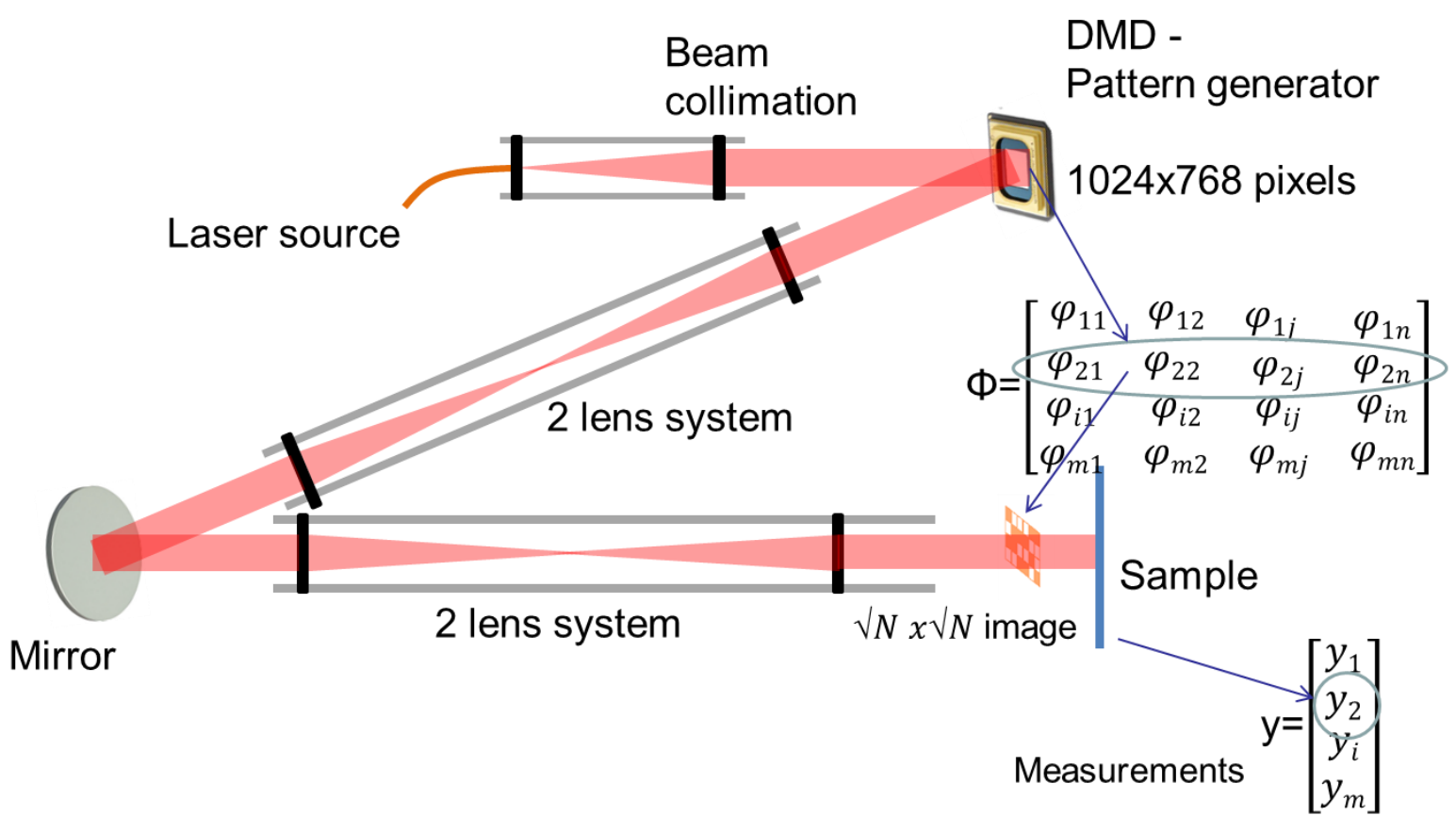

Figure 5.1. Schematic diagram of the first version CS current mapping experimental setup at NPL developed in this work.

2-lens systems with spatial filters are used for rejecting out of focus, diffracted beams and cleaning the image to be projected. This is necessary as diffraction from the DMD may be minimised but is still present. Achromatic doublets of $25.4 \mathrm{~mm}$ lens diameter were used. These 2-lens systems also create the projection of the pattern onto the sample. However, the pinhole should not be extremely small, as it has to include the first order high frequencies, 
or else the projection will lack sharpness and the reconstructed current map will appear more blurred. The sample is placed vertically with a sample holder at the focal length of the last achromatic doublet. Electrical measurements of the PV cell's current response are achieved using a Vinculum SP042-series current-to-voltage amplifier while the voltage output is measured using a National Instruments NI USB 6211 AD converter.

Using an $\mathrm{x}-\mathrm{y}$ translation stage for a raster scan, some milliseconds would be required to move from one point to the next. A significant advantage of using the DMD is that its response time is under $20 \mu \mathrm{s}$, a property that can result in increased measurement speed, which would be only limited by the sampling rate and the number of samples taken for each pattern. The sampling speed of this initial system in this series of experiments was 2 samples (patterns) per second. Thus, when taking half the measurements a point by point scanning system would need (50\%) and considering that a $100 \times 100$ pixel image (10000 pixels) is acquired, measurements last approximately 40 minutes. The main reason for the limited sampling speed was that the current measurement of this system was slightly noisy and not very accurate due to the low reading accuracy of the Vinculum SP042. A reference measurement is also acquired for monitoring laser light intensity. The maximum laser intensity on the sample that can be achieved is approximately $100 \mathrm{~W} / \mathrm{m}^{2}$ for the $658 \mathrm{~nm}$ laser and $300 \mathrm{~W} / \mathrm{m}^{2}$ for the $785 \mathrm{~nm}$ one, considering the illuminated areas of the sample. The experimental results presented in this work are all acquired with these irradiance levels on the sample, for an illuminated pixel. Although the laser sources have relatively high power and could theoretically provide higher irradiance on the sample, there are a lot of losses due to the expansion of the beam and the optical elements included. All measurements are normalised with the reference measurement, in order to correct for any laser instability.

The experimental compressive sampling procedure is also included in Figure 5.1. The projected binary patterns that apply compressive sampling are physically projected on the sample with the utilisation of the DMD. The current response of the PV device is measured for each pattern, thus populating measurement vector $y$, which is also illustrated in the figure. The patterns-test functions populate sensing matrix $\Phi$ and the required current map in vector form $\boldsymbol{x}$ is acquired by solving the resulting underdetermined optimization problem. The discrete cosine transform is applied as a basis $\psi$ to provide the sparse representation of the signal. Reconstruction of the final current map is implemented in MatLab, using the $\mathrm{l} 1 \mathrm{MAGIC}$ package [15]. 


\subsection{Initial experimental results}

\subsubsection{Multicrystalline Silicon Sample}

An $8 \mathrm{~cm}$ by $8 \mathrm{~cm}$ ribbon mc-Si cell was encapsulated and characterised at CREST in order to be used as a sample for CS current mapping with the experimental setup at NPL and be durable enough to be exchanged between the laboratories. Results using this sample are presented to evaluate this first version of the experimental setup. The sample contained a lot of defects, as can be seen from the EL image presented in Figure 5.2. Characteristic twin boundaries typical for ribbon silicon cells are also visible [43].
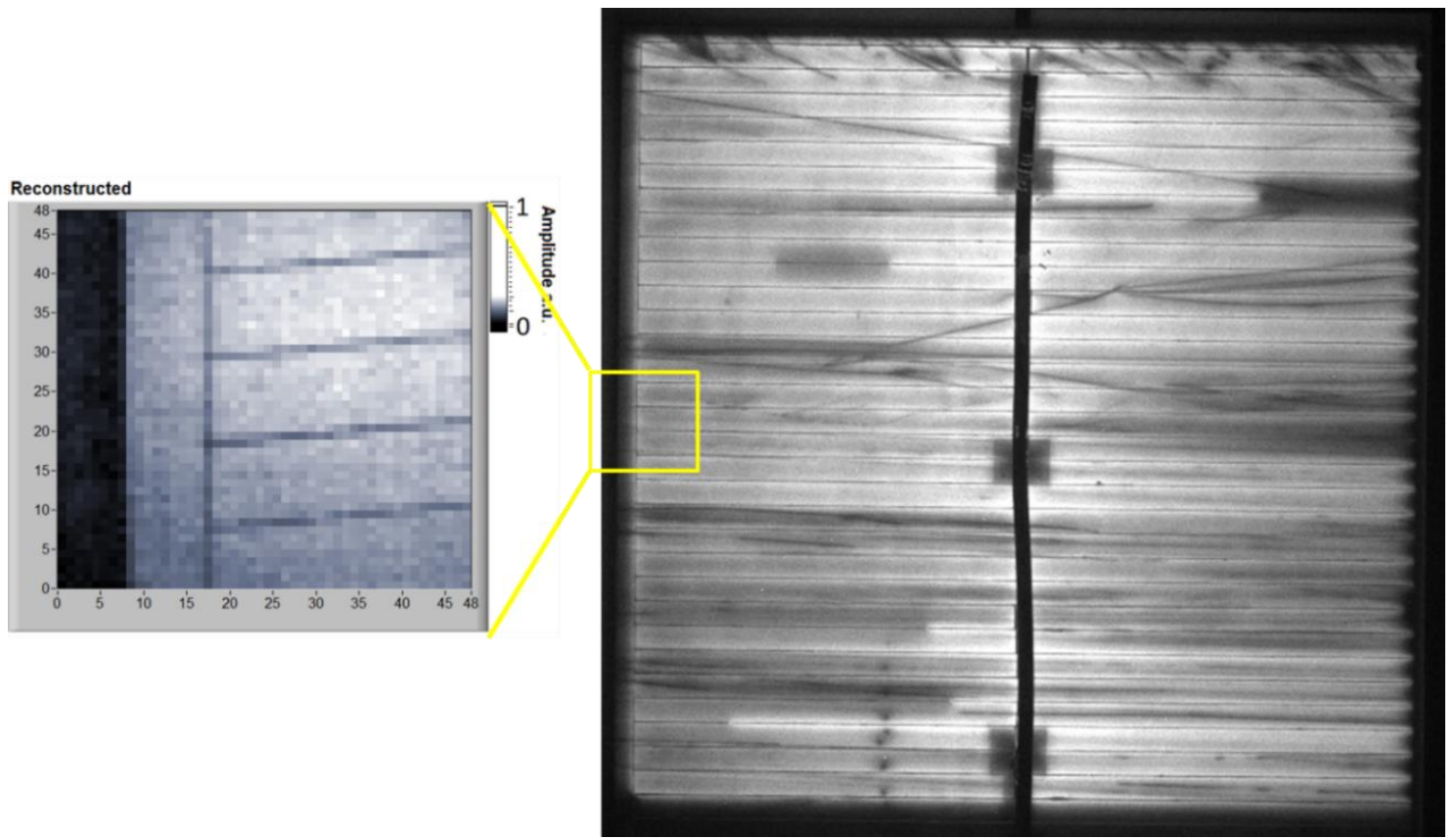

Figure 5.2. EL image of the mc-Si encapsulated cell used for CS-current mapping (right) and a small area $48 \times 48$ pixel CS current map at the edge of the sample, delivered by the prototype CS setup (left).

Initially an area at the edge of the sample was measured, as presented in Figure 5.2. The sharp transition from the active area to the area outside of the sample gave a first reference point to detect, which helped to confirm the functionality of the setup and optimise alignment of the measurement setup. The $658 \mathrm{~nm}$ laser was used at this stage. By grouping different numbers of the micro-mirrors of the DMD together, current maps with several levels of resolution can be realised. Although the DMD consists of $1024 \times 768$ pixels, a $768 \times 768$ area of the DMD was used for creating a square projection for simpler analysis. As a result, current maps of $48 \times 48,64 \times 64,96 \times 96$ and $128 \times 128$ pixels were acquired with the current 
setup. For instance, when acquiring measurements with $48 \times 48$ resolution, $265(16 \times 16)$ micromirrors are binned together for each pixel, producing a current map of 2304 pixels. The same approach is followed for the other levels of resolution. Depending on the number of measurements implemented, maps up to $64 \times 64$ need several seconds to reconstruct using the $\ell 1$ minimisation algorithm, while achieving higher resolution requires several minutes. This does not include measurement time but only data processing when sampling has finished. For the initial step of alignment and optimisation, $48 \times 48$ maps of the area at the edge of the sample were acquired.

When using the $658 \mathrm{~nm}$ wavelength laser, light does not penetrate deep into the cell and mainly the surface is scanned, rather than the bulk. This means that internal cracks, grain boundaries and defects of the cell that are visible in the EL image cannot be detected. The 48 $x 48$ current maps of a small area of the cell using this laser are presented in Figure 5.3. The image consists of 2304 pixels and current maps reconstructed acquiring different numbers of measurements are presented as a percentage of the total number of pixels of the current map (i.e. $100 \%$ being the number of measurements a raster scan would need). For instance, the 30\% current map was produced with 691 measurements. The measurements acquired with compressive sampling will be expressed as a percent throughout this chapter, following this logic.

In this first low resolution set of measurements several issues are noticed. The Gaussian top-hat distribution of the irradiance of the beam is obvious in the current maps of Figure 5.3. In addition, the fingers of the sample do not appear perpendicular to the side finger as they should be, but seem to have an inclination. This is because the projection plane does not exactly coincide with the plane of the sample, thus the image appears distorted, as if it were observed from an angle. Although the collimated beam is incident on the DMD perpendicularly, the patterns are inserted into the optical system by an angle and this is the reason for this specific artefact. Despite these artefacts in the final images, current maps are producible by acquiring only $40 \%$ of the measurements a raster scan would need. Below $20 \%$ there is not enough information for a proper reconstruction of a current map, but adding more measurements from that point the features of the sample start to become visible. Above $40 \%$ no significant improvement of the images is noticed. No other features except the edge of the sample and its finger are visible, due to the short wavelength of the laser having limited penetration into silicon and the low resolution selected. 


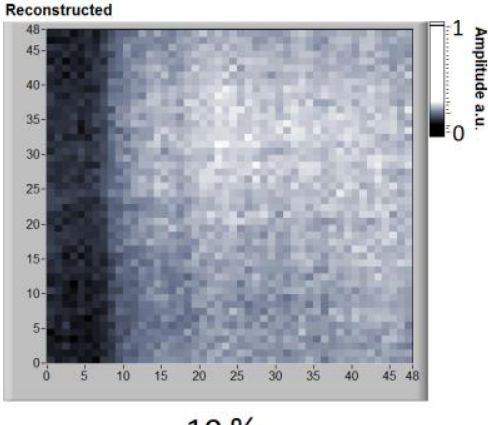

$10 \%$

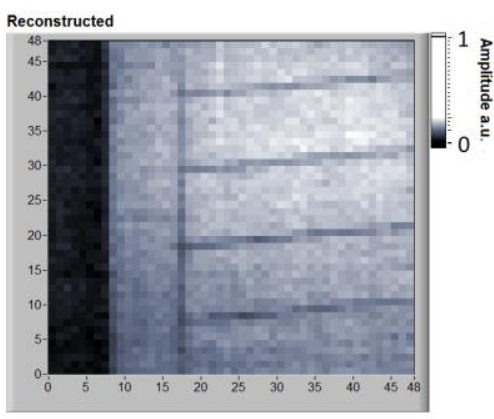

$40 \%$

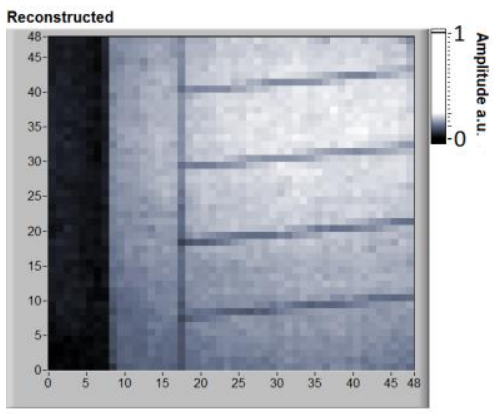

$70 \%$

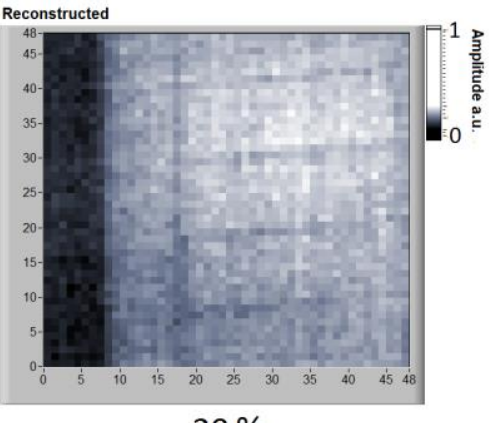

$20 \%$

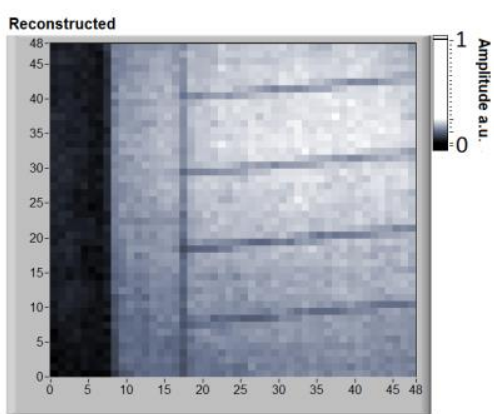

$50 \%$

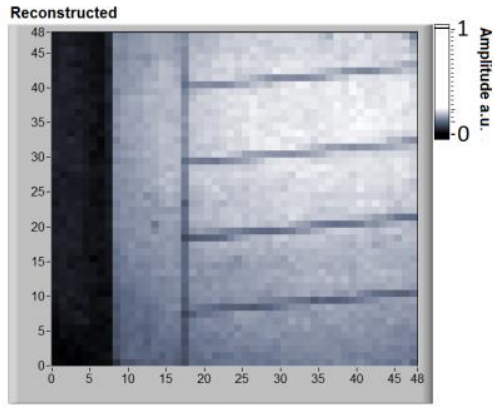

$80 \%$

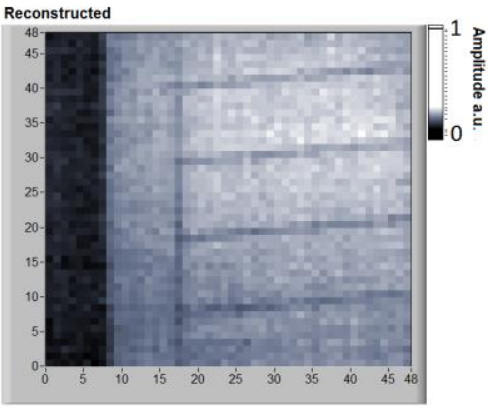

$30 \%$

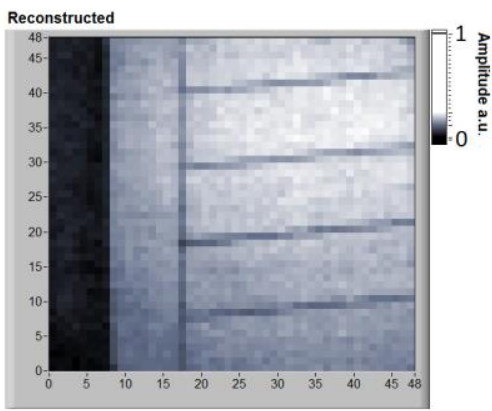

$60 \%$

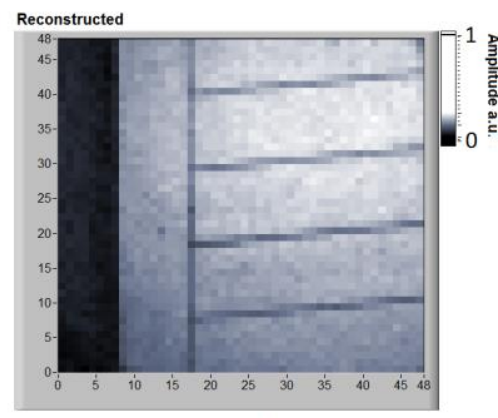

$90 \%$

Figure 5.3. $48 \times 48$ pixel CS current maps at the edge of the sample, delivered by the prototype CS current mapping setup. The percentages represent the ratio of the number of measurements to the total pixels of the image. The current signal is expressed in arbitrary units.

To penetrate deeper into the PV sample, the $785 \mathrm{~nm}$ laser source was used and an area of the PV sample with a crack was measured, as presented in Figure 5.4. Penetration depth at this wavelength is not more than a few micrometres, however, it is adequate for detecting the cracks visible in the EL images. The same artefacts as before are apparently present in the reconstructed current map: imperfectly uniform irradiance on the sample and the difference between the plane of the projection and the plane of the sample distorting the current map, making the fingers look inclined and not perpendicular to the busbar.

Measurements with several levels of resolution using this laser wavelength were realised for this small $1 \mathrm{~cm} \times 1 \mathrm{~cm}$ area containing a crack and two very small spots. The lowest 
resolution was $48 \times 48$ pixel images, corresponding to a resolution of $220 \mu \mathrm{m}$ and the highest $128 \times 128$ pixel maps, corresponding to an $82 \mu \mathrm{m}$ resolution, considering that each micromirror has a size of $13.7 \mu \mathrm{m}$ by $13.7 \mu \mathrm{m}$. Increasing optical resolution makes the features of the sample such as fingers, cracks and spots become sharper.

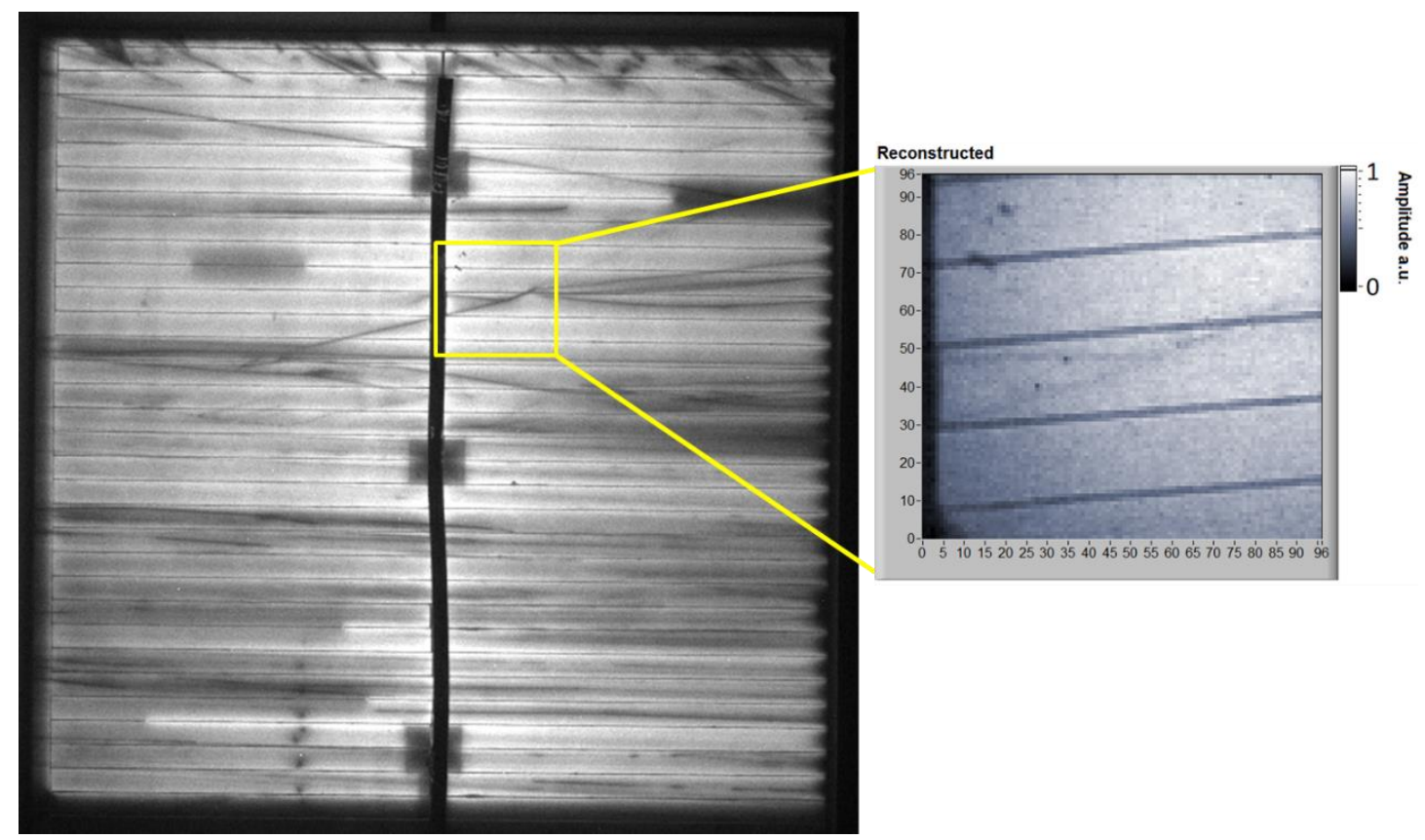

Figure 5.4. EL image of the mc-Si encapsulated cell used for CS current mapping (left) and a small area $96 \times 96$ pixel CS current map at an area of the sample where a crack is present, delivered by the prototype setup (right).

In the $48 \times 48$ pixel images presented in Figure 5.5 the crack is barely visible, which is expected at this level of resolution. However when increasing optical resolution the defects of this small area become visible, as presented in the $96 \times 96$ pixel current maps (9216 pixels) of Figure 5.6. In this case the crack and the small spots are visible already with 2765 measurements (30\%) and above 3787 measurements (40\%) there is no significant improvement of the current map quality. Above 8294 measurements (90\%) measurement noise starts to affect the reconstruction process, making the reconstructed current map rather noisy. When acquiring 9216 measurements (100\%), which is what a point by point scan would need, the reconstruction process fails completely. The $99.9 \%$ reconstruction current map included in Figure 5.6 shows that no useful results are acquired when approaching $100 \%$ of measurements. This was expected from the predictions in the previous chapter, where noise was inserted in the simulation process. 


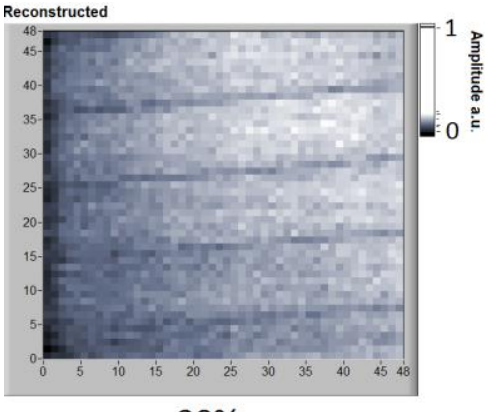

$20 \%$

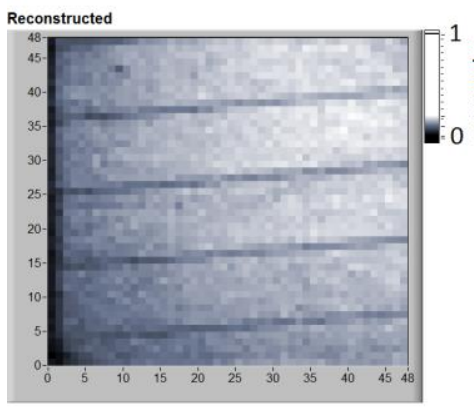

$50 \%$

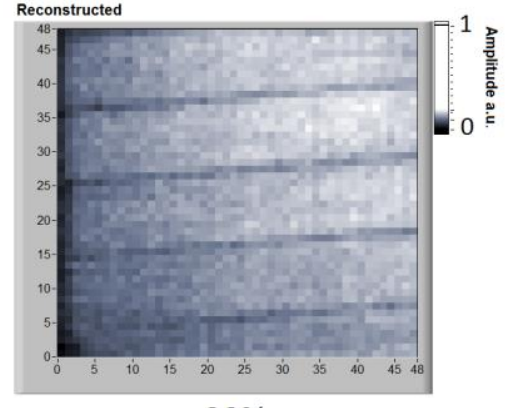

$30 \%$

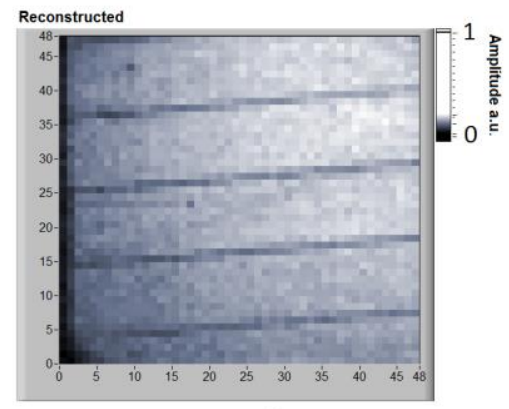

$60 \%$

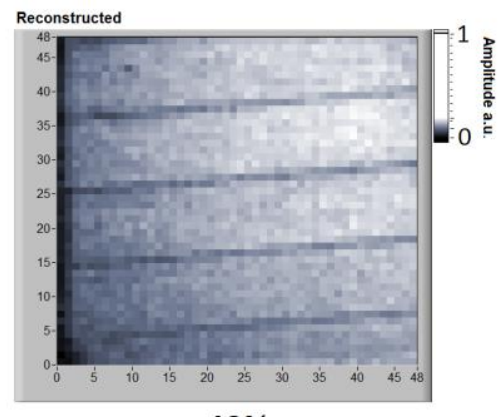

$40 \%$

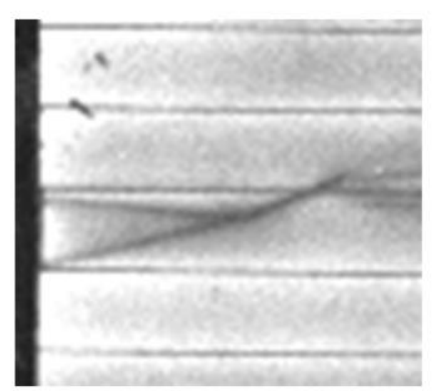

EL image

Figure 5.5. $48 \times 48$ pixel CS current maps at the area of the sample where a crack is present, delivered by the prototype CS current mapping setup. The percentages represent the ratio of the number of measurements to the total pixels of the image. The EL image of this area is also included for comparison. The signal is expressed in arbitrary units.

By considering only that fewer measurements are needed for a current map, this first series of measurements shows that a reduction of solar cell current mapping measurement time using this approach is realistic. A CS current mapping system would require less than half of the time of what a standard LBIC system would need to scan the same area with the same resolution, assuming the same sampling speed for both cases. This on its own is a significant improvement compared to standard LBIC measurements. Above $90 \%$ measurements, noise begins to impact on the optimisation algorithm that reconstructs the images, thus the image is contaminated with random noise. It is clear from the results in Figure 5.6 that for this level of resolution the highest quality images are delivered in the range of $40 \%$ to $80 \%$ of measurements. However, as discussed in the previous chapter, this optimum range will depend on the amount of noise present in the measurement process. In reality, this will be determined by the design of the measurement setup, the sampling equipment and the sample under measurement. 

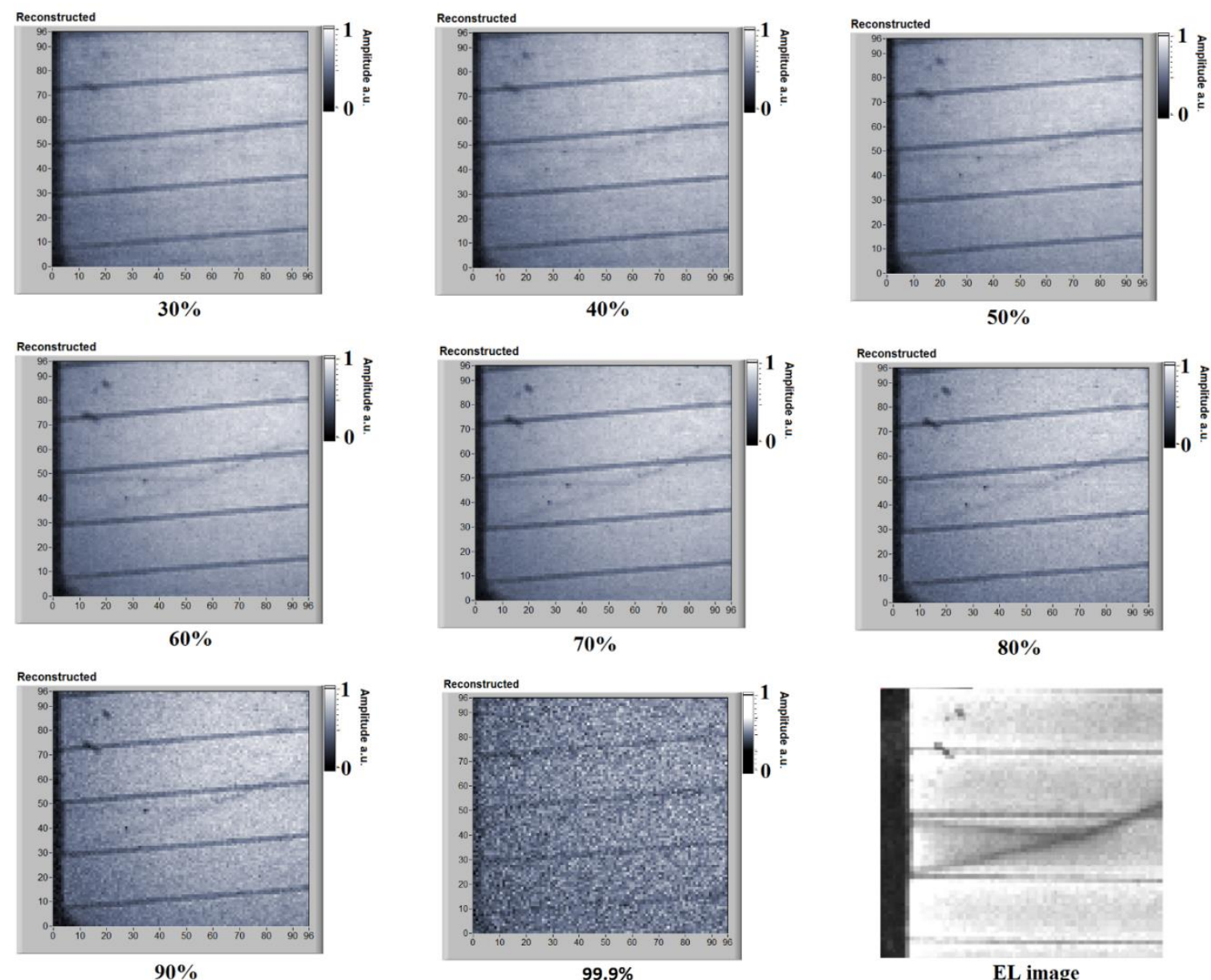

EL image

Figure 5.6. $96 \times 96$ pixel CS current maps at the area of the sample where a crack is visible, delivered by the prototype CS current mapping setup. The percentages represent the ratio of the number of measurements to the total pixels of the image. All the features of the cell's area are clearly visible above $40 \%$ of measurements. The signal is expressed in arbitrary units.

In Figure 5.7, high resolution 128 x 128 pixel (16384 pixels) reconstructed current maps with different numbers of measurements acquired are presented, alongside the EL image of the same area for comparison. The defects present in this area of the sample become visible already from 25\% of measurements (4096 measurements) upwards and adding more measurements results in a sharper reconstruction. The $40 \%$ map already provides a very high resolution current map, showing the power of the compressive sampling method, even with this initial experimental setup. Adding more measurements offers no real improvement of the current map. It is also evident that higher resolution reconstructed current maps converge faster to the real solution, which is consistent with the findings of section 3.4.2. This means that for large-scale current mapping, compressive sampling is more favourable than point by point sampling and has the potential for even larger measurement time reduction. 

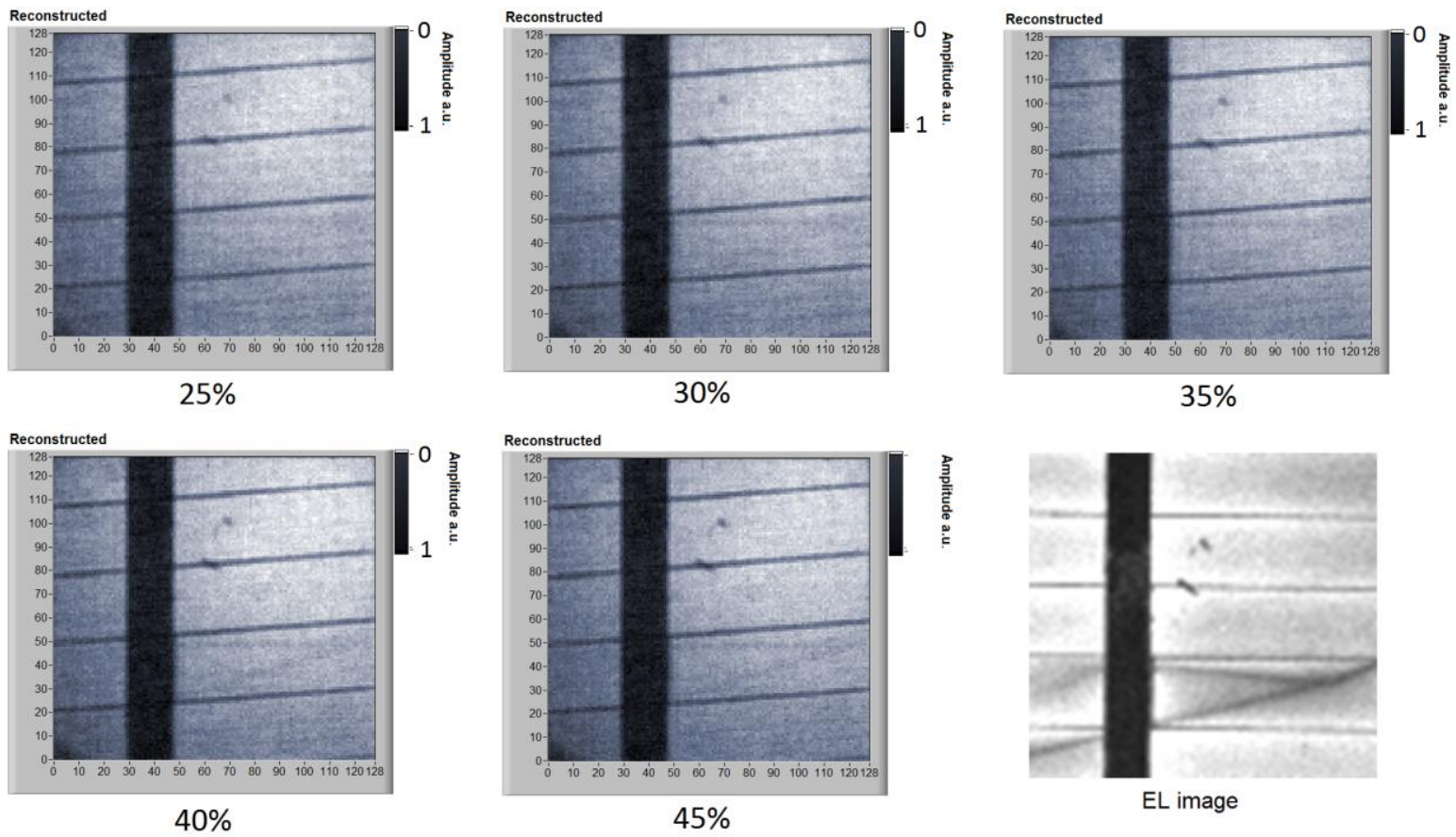

Figure 5.7. $128 \times 128$ pixel CS current maps at the area of the sample where a crack is present, delivered by the prototype CS current mapping setup. The percentages represent the ratio of the number of measurements to the total pixels of the image. The EL image of this area is also presented for comparison

The measurement results of this experimental approach confirm the functionality and the potential of the CS current mapping technique, and validate the simulation results of the previous chapters. Nevertheless, some improvements were identified so this setup was optimised and some of the concerns eliminated.

\subsubsection{Thin film sample}

A thin film sample was also used for measurements with the initial setup. The sample is a $2 \mathrm{~mm}$ by $3 \mathrm{~mm}$ solution processed CIGS cell [31] produced at CREST. The $658 \mathrm{~nm}, 40 \mathrm{~mW}$ single mode fibre coupled laser is used for measurements in this case. CS current mapping results are presented in Figure 5.8, with an LBIC map of the same sample for comparison, acquired with the LBIC system in CREST with a laser wavelength of $635 \mathrm{~nm}$ [107]. The CS current maps have a display resolution of $100 \times 100$ (10000) pixels and different levels of optical resolution were realised. This can be achieved easily by grouping a smaller number of micromirrors of the DMD chip together and measuring a smaller area of interest on the 
sample. The different pixel resolutions in Figure 5.8 were realized by binning $5 \times 5,3 \times 3$, and $2 \times 2$ pixels of the DMD together for control. 5000 (50\%) measurements were acquired to produce the current maps of Figure 5.8 as they provide a reliable reconstruction as shown in previous results. An optical resolution of $27 \mu \mathrm{m}$ is achieved without any demagnification optical elements. Measurements are made here on an area of interest with a large local defect on the top left area of the cell, to provide more information on current non-uniformities of that specific area.

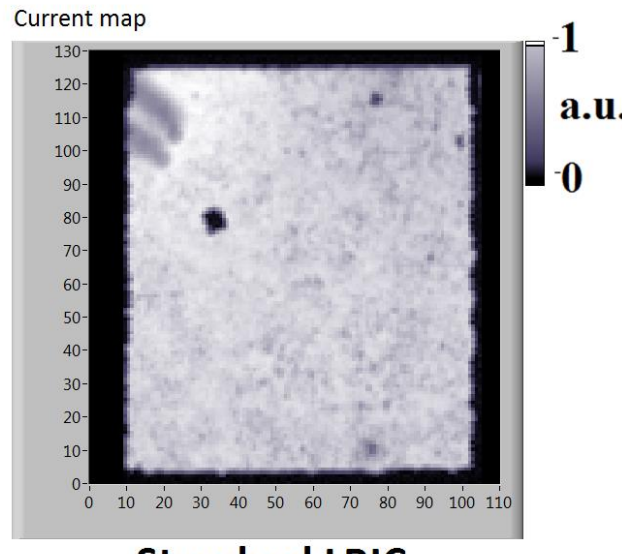

Standard LBIC

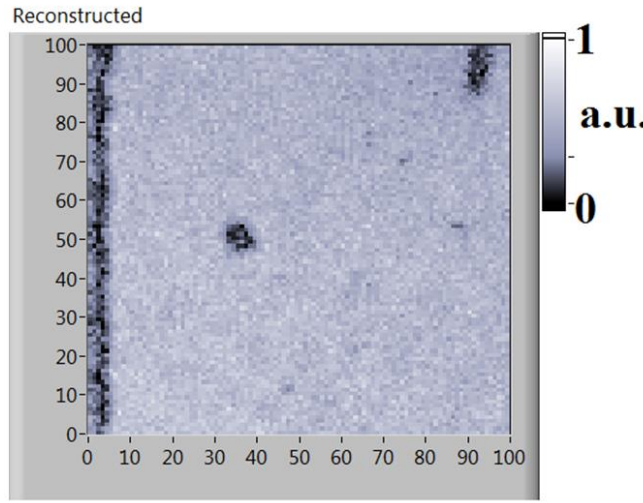

CS 50\% $41 \mu \mathrm{m}$

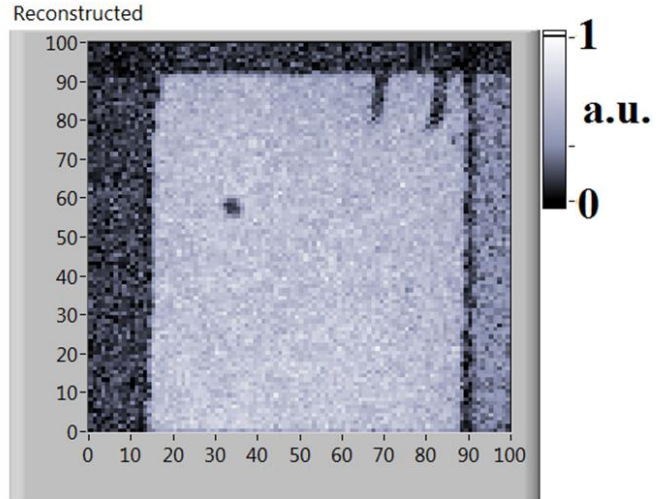

CS $50 \% 67 \mu \mathrm{m}$

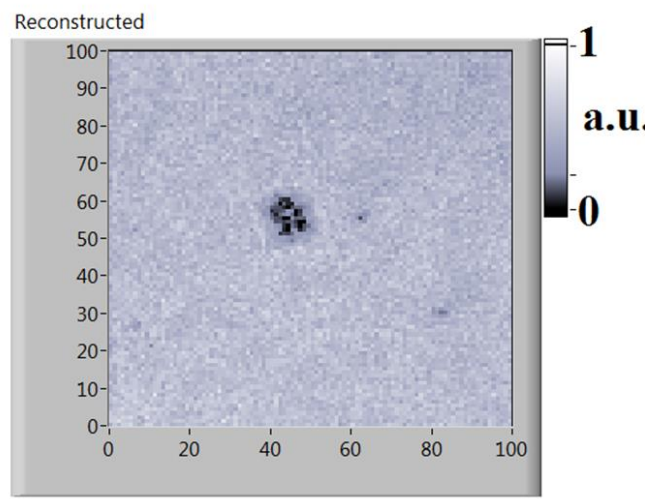

CS 50\% $27 \mu \mathrm{m}$

Figure 5.8. $100 \times 100$ pixel CS current maps of the area of the sample where a pinhole is present. The percentages represent the ratio of the number of measurements to the total number of pixels of the image. An LBIC scan of the same sample is also presented for comparison (top left). The pixel size is also noted for each CS current map.

The small device under test was produced in-house at CREST and consists of several different cells on the same substrate. However, the cells are not perfectly isolated and signal from adjacent cells is also measured as can be seen by the CS current maps. The probes used 
for contacting are clearly seen on the top right corner of the CS map and the top left corner of the LBIC map in Figure 5.8. As measurement points do not overlap each other when using compressive sampling, there is some pixelation in very uniform areas of the cell in the CS current maps. Additionally, the sample exhibited serious preconditioning issues. When it was illuminated, its stabilisation time was in the range of several seconds. This inserted significant amounts of noise in the measurements, which is clear in the reconstructed images, especially when comparing the results with those of the mc-Si sample.

CS current maps of the area with the defect with different numbers of measurements acquired are presented in Figure 5.9. Acquiring more than 5000 (50\%) measurements provides no further improvement of the current map, while even 4000 measurements provide a reliable map of the sample. The performance of the method for this thin film device is similar to that for the mc-Si sample case, with the useful region of undersampling being in the range of $40 \%$ to $80 \%$. Even with this initial experimental setup, these results confirm that the CS current mapping method has similar performance for different types of PV samples.
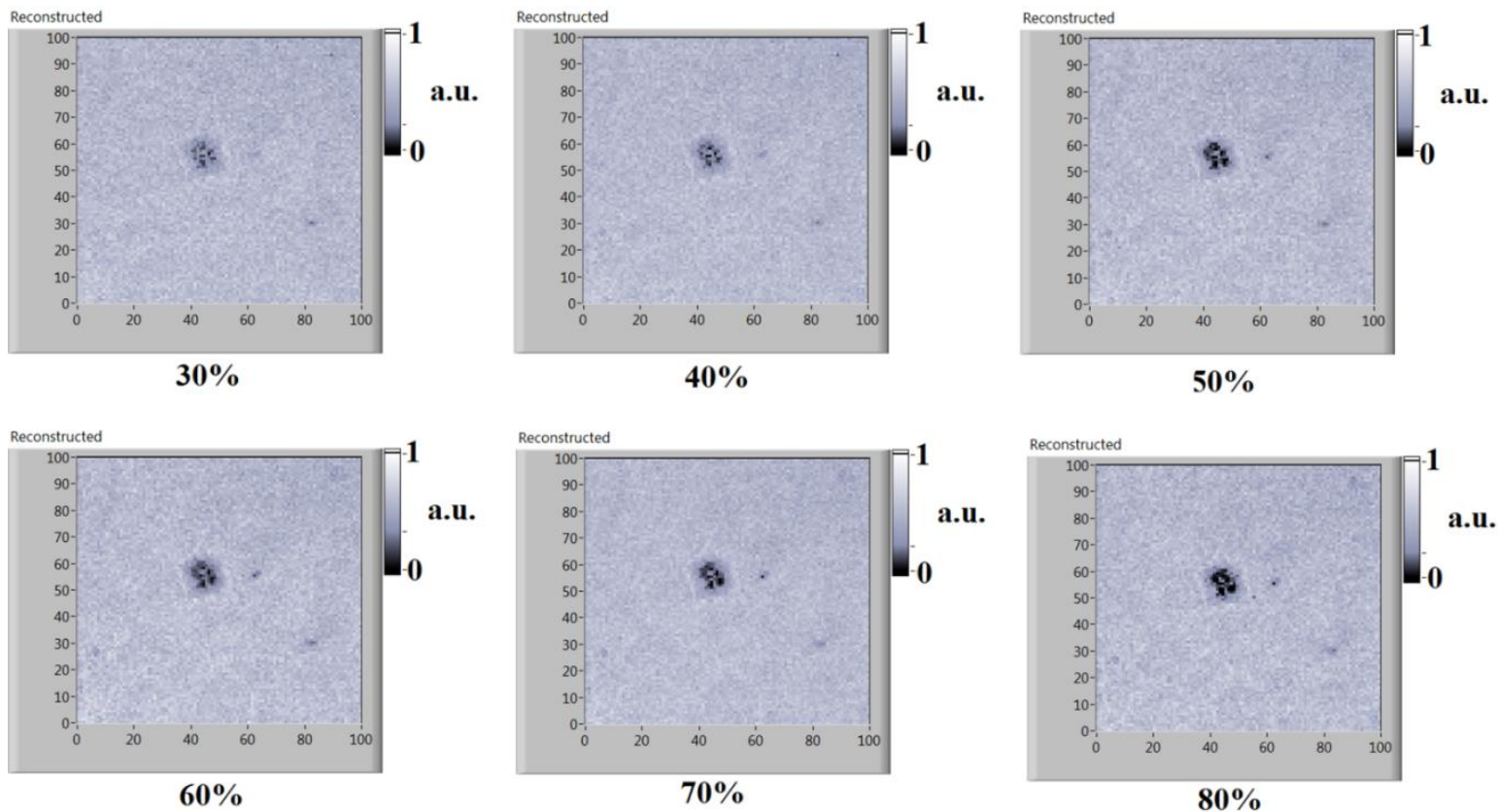

Figure 5.9. $100 \times 100$ pixel CS current maps at the area of the thin film sample where a pinhole is present. The percentages represent the ratio of the number of measurements to the total number of pixels of the image. 


\subsection{Optimised CS current mapping setup}

\subsubsection{Optical System design}

The approach for the optimised CS current mapping setup considers the issues identified in the initial implementation of the previous section. A major improvement is that the setup is significantly simpler and more straightforward to realise with specific differences from the previous approach; larger achromatic doublets are used, with a lens diameter of $50.8 \mathrm{~mm}$, for reducing lens distortion effects; the sample is placed horizontally on a z-stage platform; a more accurate current measurement instrument is used. The setup is analytically described below. With the enhanced optical and measurement performance of this implementation, both compressive and point by point sampling modes were realised successfully so that a direct pixel by pixel comparison between the two sampling methods is possible.

The experimental setup is presented in Figure 5.10. The available laser sources are the same as before; a $40 \mathrm{~mW}$ laser at $658 \mathrm{~nm}$ wavelength and a $100 \mathrm{~mW}$ laser at $785 \mathrm{~nm}$. Both sources are single mode fibre coupled. The light output of the fibre is collimated at a size so that the beam overfills the micro-mirror array (DMD). The DMD is the same V-7000 module, consisting of a $1024 \times 768$ pixel micromirror array, each micromirror having a pixel size of $13.7 \mu \mathrm{m} \times 13.7 \mu \mathrm{m}$. In order to insert the CS patterns into the optical system without any distortion, the collimated beam is incident on the DMD at an angle in this revised setup. Another small benefit is that the beam projection onto the DMD chip has a larger area. A central region of this area is used, which leads to a slight enhancement of final projection uniformity, compared to the previous implementation. The plane of the micromirror area is perpendicular to the spatial filter system. This eventually leads to the coincidence of the plane of the projected pattern and the plane of the sample for this setup. However, as the DMD consists of finite elements (micromirrors) and the beam is incident at an angle, diffraction of the beam is inevitable. To minimise diffraction, the incident angle is set to the blaze angle of the DMD [202]. The blaze angle is wavelength dependent and within a very small range, so precise alignment is necessary to achieve it for the different laser wavelengths. Using the blaze angle, diffraction is suppressed but not completely eliminated. Therefore the spatial filter is once again used to reject diffracted and non-collimated components of the beam. This is necessary for a much clearer laser spot or pattern projection onto the sample. Finally, a mirror is used for guiding the beam onto the sample, which is placed horizontally on a z-stage 
platform. This is a much more convenient approach for placing all types of samples and provides a higher degree of accuracy in positioning the sample in the projection plane.

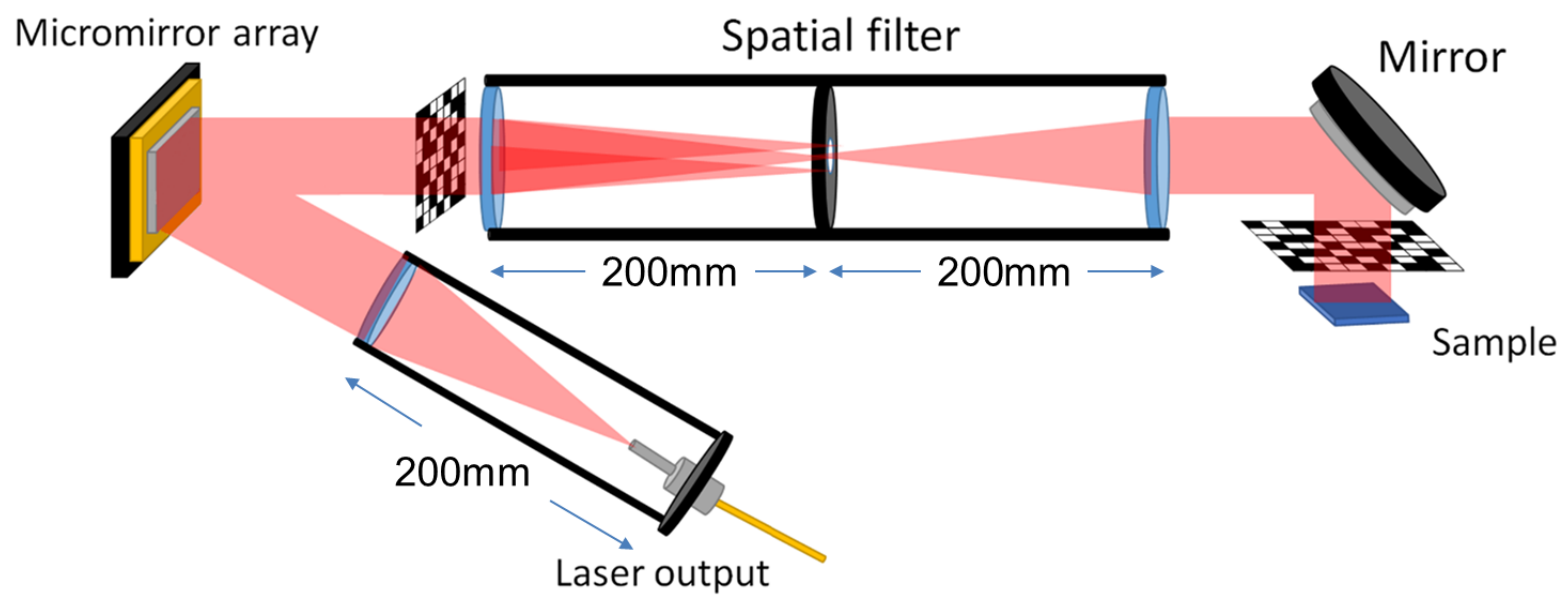

Figure 5.10. Schematic diagram of the DMD based current mapping experimental setup. The random patterns used for the case of CS current mapping are also included in the picture. The diagram is not drawn to scale.

A National Instruments PXle-4139 system source measure unit (SMU) is used for measuring the current response of the sample. This instrument provides much higher accuracy than the previous measurement setup, being able to measure current even in the $\mu \mathrm{A}$ range with $\mathrm{pA}$ resolution. Pictures of the experimental layout are presented in Figure 5.11Figure 5.10 and Figure 5.12. The experimental layout is kept as compact as possible, in order to demonstrate that the realisation of such a system is simple and that only a small amount of space and optical elements are required. The sample is placed at the focal plane of the last lens, so that the scanning spot or the patterns are actually projected on the sample. Nevertheless, a small misplacement from this plane has almost no effect on measurements due to spatial filtering. The optical setup is suitably enclosed for minimising external light contamination as well as for laser safety reasons, as it is illustrated in the photographs of Figure 5.13. 


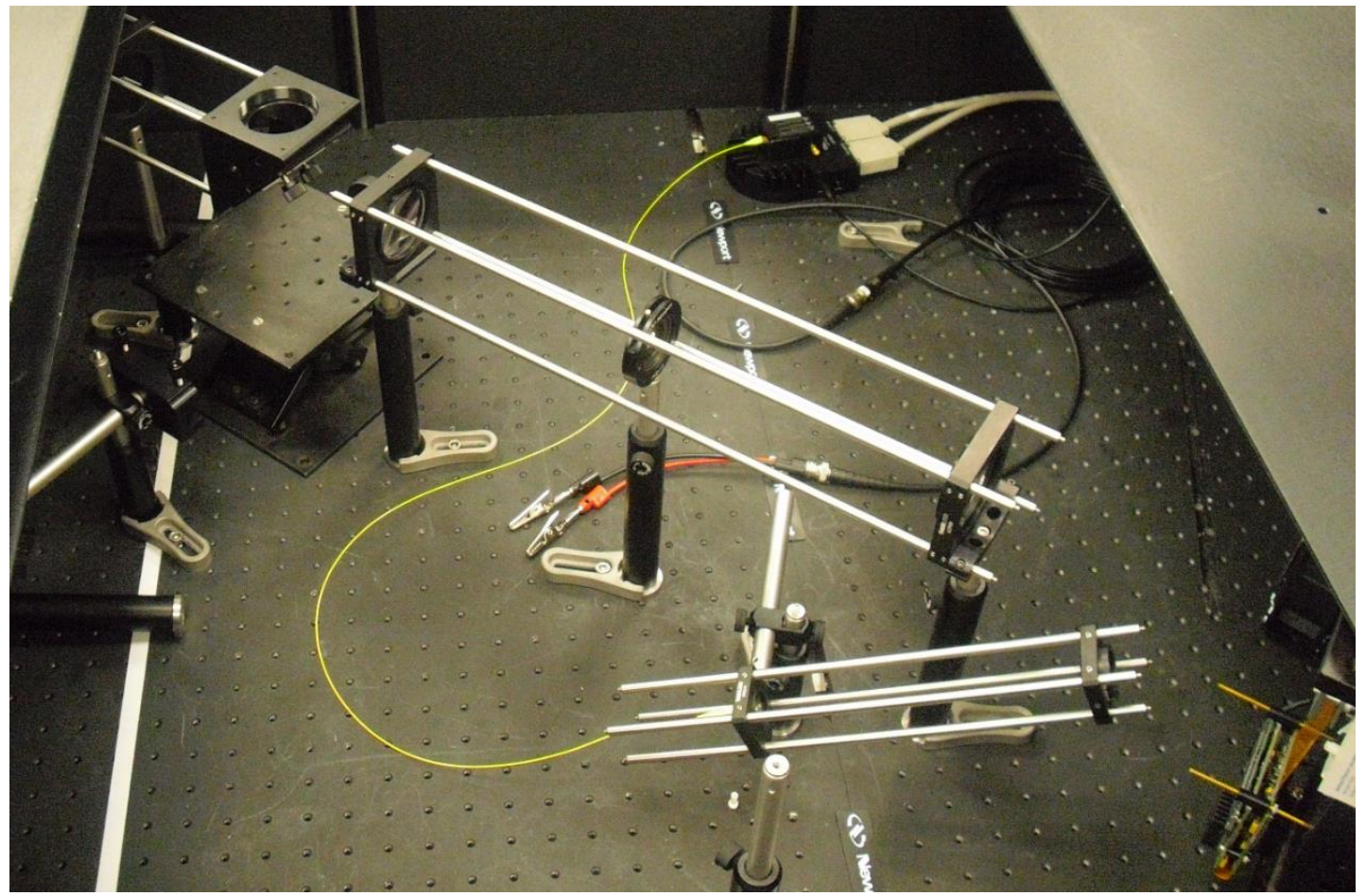

Figure 5.11. The optimized version of the current mapping setup based on the DMD chip developed at NPL.

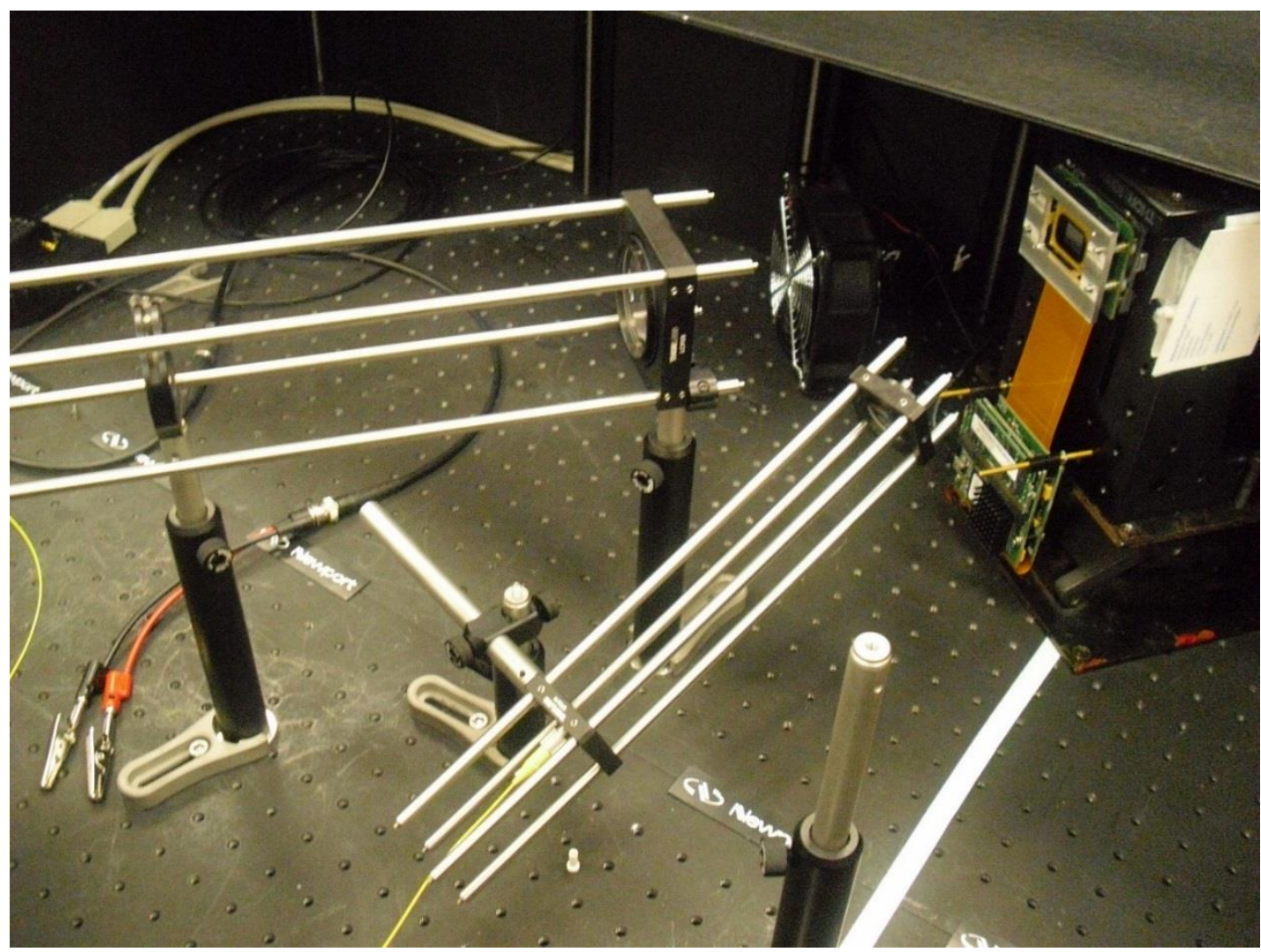

Figure 5.12. Detail of the mounting of the DMD and the laser launch and collimation. The collimated beam is incident on the micromirror area at an angle. 

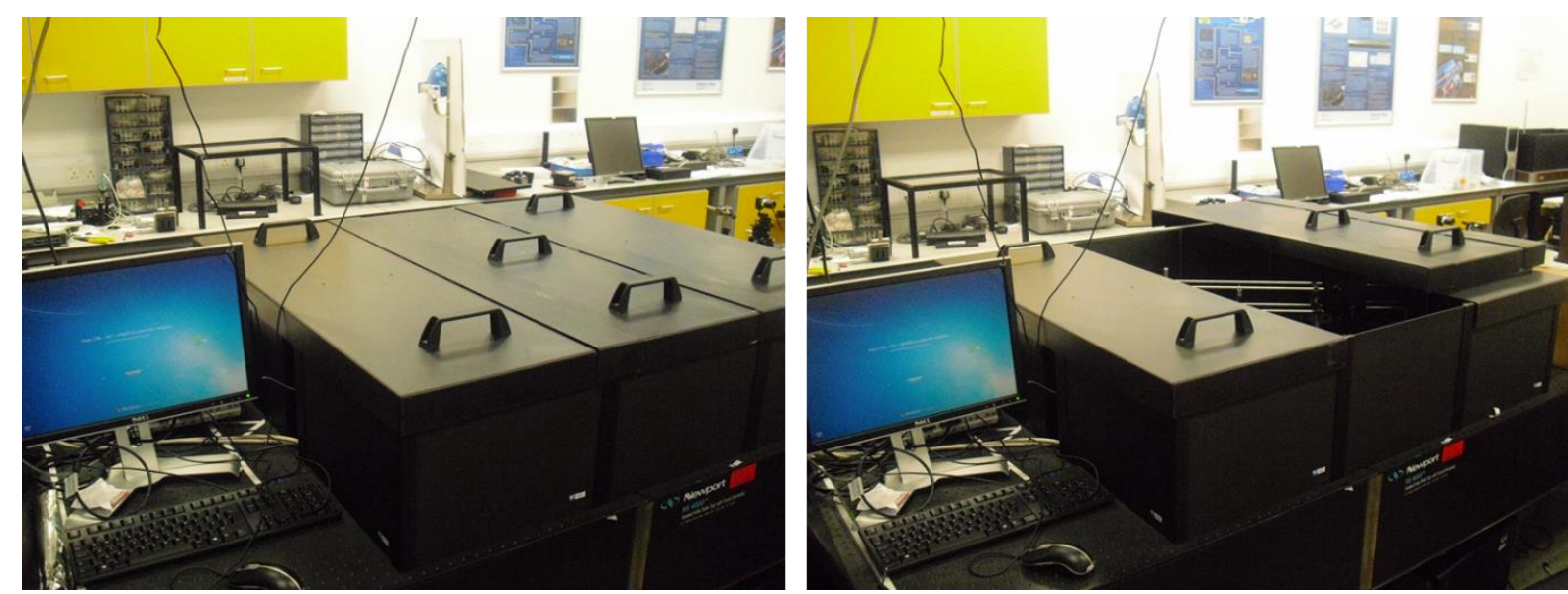

Figure 5.13. The enclosure and workstation of the CS current mapping optical setup developed in this work. The cover of the setup consists of three pieces with handles for easier access.

In order to apply a point by point scan, a number of micromirrors are grouped together for control depending on the desired optical resolution, similar to the case of compressive sampling. It was observed that for this setup, if only one pixel of the micromirror array is used for the scan, the signal is rather low, which results in very noisy measurements. For this reason the minimum group of pixels used in this series of measurements is $9(3 \times 3)$, which results in an optical resolution of $41.1 \mu \mathrm{m}$ and the maximum is $49(7 \times 7)$, which gives a resolution of 96 $\mu \mathrm{m}$. In the case of a point by point scan the spot shape on the sample is square. $100 \times 100$ pixel current maps were acquired in the case of CS current mapping, in order to achieve a more straightforward performance analysis of the measurement system.

As previously discussed, an important advantage of using the DMD module even for a raster scan is that its response time is under $20 \mu \mathrm{s}$. This property can result in increased measurement speed, which would be limited only by the sampling rate and the number of samples taken for each pattern in the case of compressive sampling. The sampling speed of the system in this second series of experiments is set at 10 samples (positions of the spot or patterns) per second. 30 readings are actually acquired for each sample (point or pattern projected), with the average being recorded, as well as the standard deviation. This sampling speed is comparable with the speed of a typical LBIC system. However, a good LBIC system can achieve even higher sampling speed. Higher measurement speeds can also be achieved with the setup described here with optimisation of the control software, which is now still in a primitive stage.

When taking half the measurements $(50 \%)$ a point by point scanning system would need and considering that a $100 \times 100$ pixel image (10000 pixels) is acquired, measurements require approximately 8 minutes in total. A reference measurement is also acquired for monitoring 
laser light intensity. All measurements are once again normalised with the reference measurement, in order to correct for any laser instability.
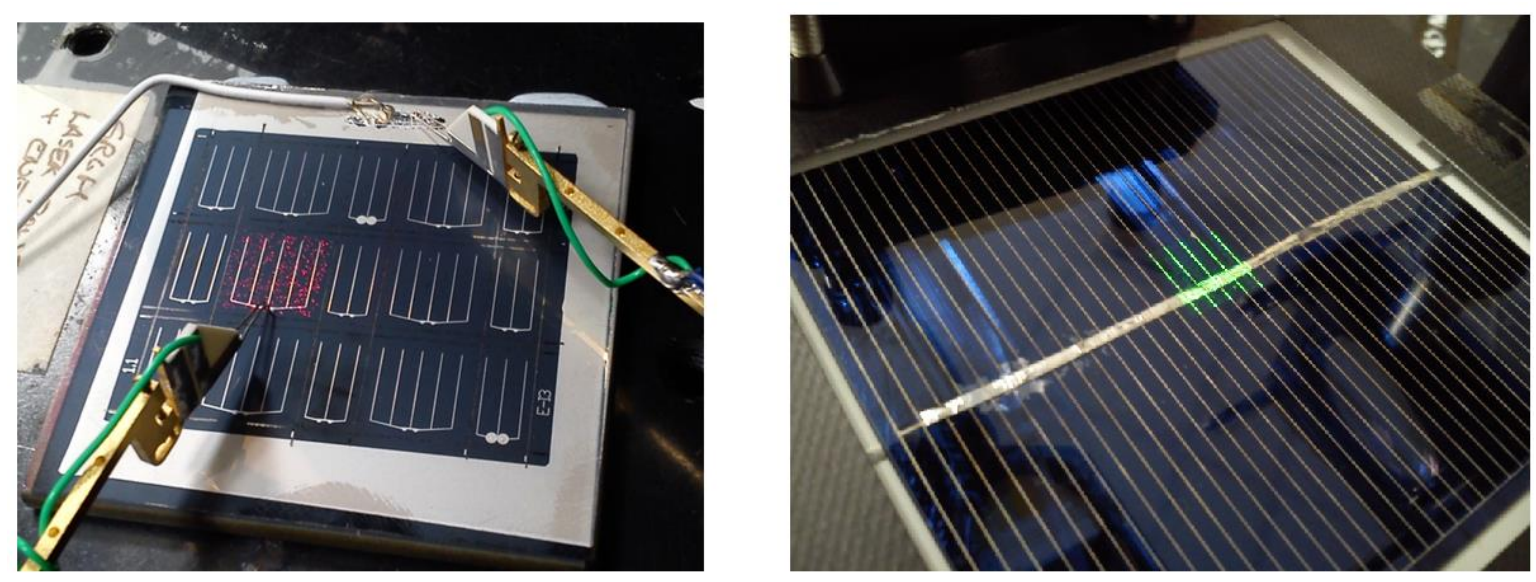

Figure 5.14. The CIGS sample on the left and the mc-Si sample on the right, which are used for measurements in this work. The CIGS sample consists of several individual cells, one of which is contacted by probes. The pattern used for compressive sampling is also visible on the samples, a $532 \mathrm{~nm}$ alignment laser is used in the picture on the right.

A different CIGS sample that consists of several individual cells is used in this series of measurements, as well as the mc-Si PV sample which was used in the previous iteration. The samples are presented in Figure 5.14, placed on the z-stage platform. The patterns projected on the PV cells under test are also visible in the picture. The individual CIGS cell used for measurements had a size of $1 \mathrm{~cm}$ by $1 \mathrm{~cm}$ and was contacted with probes. This device had no preconditioning issues so measurement noise was significantly decreased. As also mentioned above, the mc-Si cell was $8 \mathrm{~cm}$ by $8 \mathrm{~cm}$ and an area of interest of $1 \mathrm{~cm}$ by $1 \mathrm{~cm}$ was selected for measurements.

\subsubsection{Point by point scan}

As a first step, point by point current mapping was realised using this optimised setup. In Figure 5.15, two $100 \times 100$ pixel current maps of the CIGS PV cell are presented, using a different optical resolution each time and applying a point by point scan using the DMD. It is clear that by grouping different numbers of micromirror elements together one can focus on different areas of a sample with various levels of resolution. This is a very convenient feature of a DMD based system, as it allows much more freedom of resolution and scanning settings 
without the need of any additional mechanical equipment for focusing or moving the sample. In Figure 5.15, the PV cell's current response appears rather uniform, apart from local tiny spots, more visible in the higher resolution map on the right of Figure 5.15. The probe used for contacting the cell is also visible at the top of the current map. The local current values are below $1 \mu \mathrm{A}$. A slight general non-uniformity is due to the initial non-uniformity of the Gaussian collimated beam that overfills the DMD micromirror area. However, only the central part of the beam is used and the rest is rejected as it does not fall on the micromirror area, reducing this non-uniformity to less than $10 \%$ for the final current map.
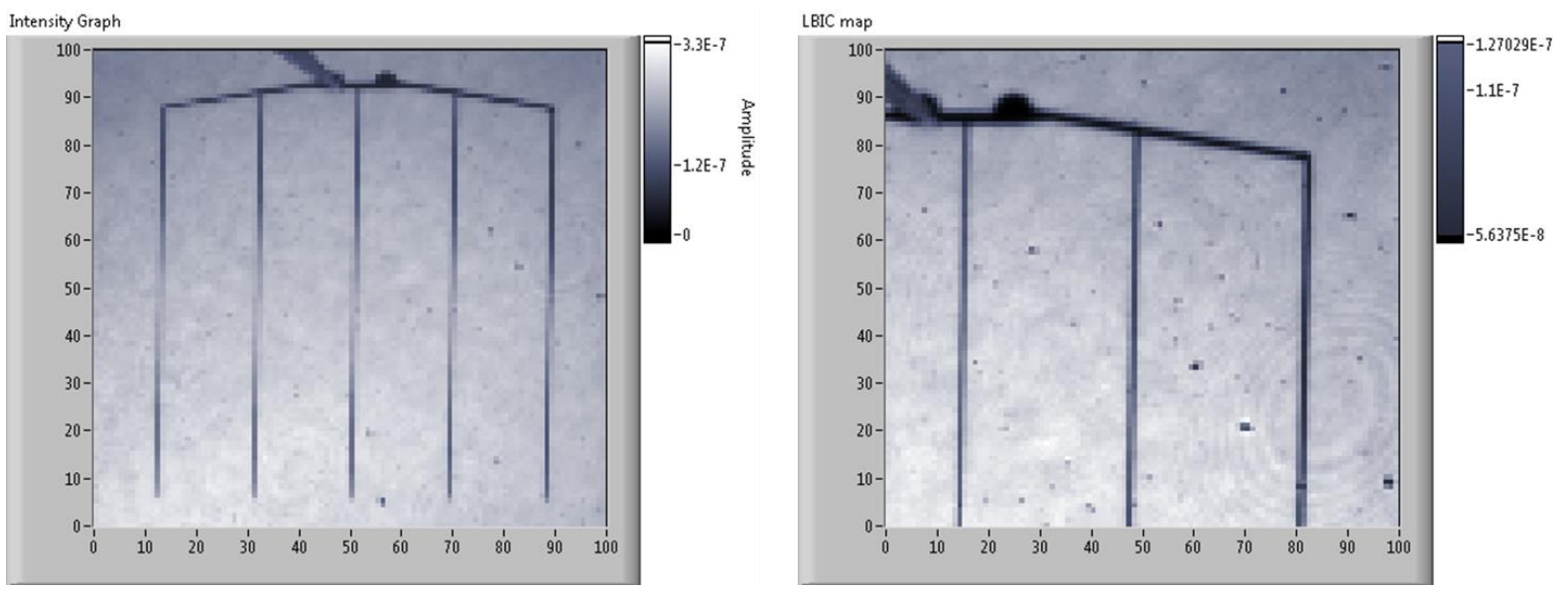

Figure 5.15. $100 \times 100$ pixel currents map of the CIGS sample. On the left, $7 \times 7$ groups of micromirrors are used for the point by point scan, measuring the whole cell. On the right, $4 \times 4$ groups are used, achieving a higher optical resolution, for a smaller area of the sample.

A high resolution scan of this individual cell of the CIGS sample is presented in Figure 5.16. $3 \times 3$ groups of micromirrors were used to create the spot for the raster scan, achieving a resolution of $41.1 \mu \mathrm{m}$. Multiple but barely visible interference patterns are present in the current response map. The source of this artefact is the multiple reflections from the surfaces of the optical elements (lenses) of the spatial filter. Such effects can be significantly reduced by using optics with high performance antireflective coatings, specifically designed for the system's laser sources. It should be highlighted that this resolution of $41 \mu \mathrm{m}$ is achieved without any demagnification optics such as a microscope objective or any other lens system. There are only three achromatic doublets used in the system, one for beam collimation and two in the spatial filter and none of them has any effect on spot size. Adding a microscope objective lens at the sample end of this system could boost optical resolution and achieve nm scale scans, if desired. 


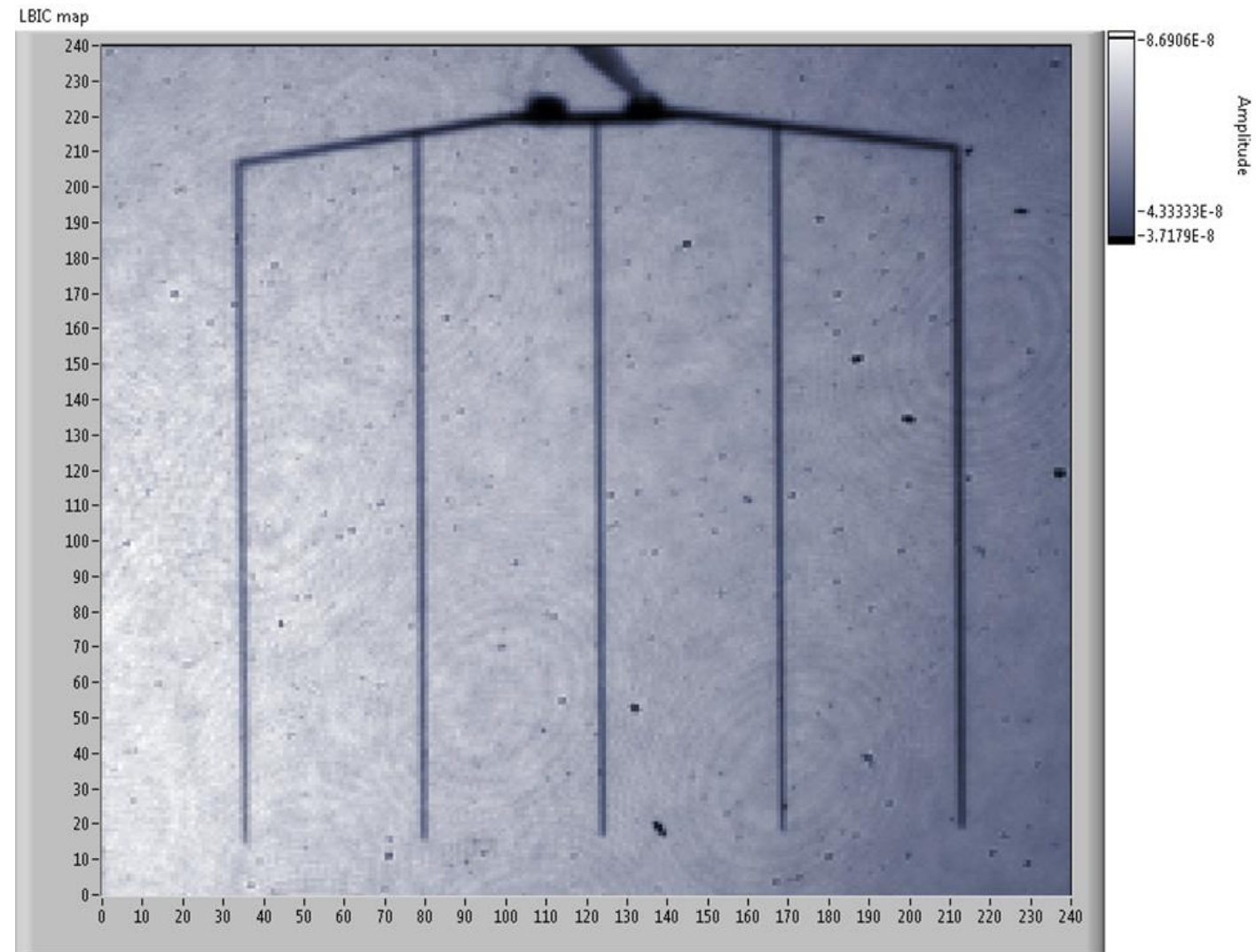

Figure 5.16. A $240 \times 240$ pixel current map of the CIGS cell, using groups of $3 \times 3$ micromirrors for the point by point scan, which provides an optical resolution of $41.1 \mu \mathrm{m}$.

It was not possible to perform point by point scans of the mc-Si sample due to the high noise levels; since the small spot used for the raster scan has a low relatively intensity and only a small area for that large area sample is scanned, the signal levels are comparable to the background noise. This was partly expected, as no lock in techniques are used, which would make the separation of the current response reading from the noise easier. Nevertheless, it is demonstrated below that current mapping of a larger sample or more generally of a sample that exhibits such high noise levels is possible. This is achieved by applying compressive sampling instead of a point by point scan, which significantly enhances signal levels.

\subsubsection{Compressive Sampling}

The same procedures as in the previous implementation were followed in this instance. $7 \times 7$ groups of micromirrors were used and $100 \times 100$ pixel current maps were produced. This resolution was selected for easier data analysis and comparisons with the point by point scan 
case. For the case of the CIGS sample the whole area of an individual PV cell is measured. As the aim of compressive sampling is to apply fewer measurements in order to reconstruct the final current map, different levels of undersampling were investigated. The unique property of the experimental layout demonstrated in this work is that it can apply both point by point and CS current mapping, which means a pixel by pixel comparison can be made, for a more accurate and quantitative experimental evaluation of CS current mapping. This is the first time that an optical system has been built that can perform both compressive and point by point sampling, making it an ideal instrument for investigating reconstruction algorithms and sensing matrices for experimental CS applications.

In Figure 5.17, the reconstructed current maps are expressed as a percentage, the ratio of samples (projected patterns) acquired for reconstruction to the total number of pixels or equivalently, the number of measurements the LBIC scan needs, to provide the current map. The total number of pixels of the current maps is 10000 . The main features of the PV cell become visible from 3000 measurements (30\%) while adding more and more measurements the result converges to the point by point current map. Above $70 \%$ the improvement is marginal, similar to previous results.

Some pixelation effects are very similar to the case of the simulations for the uniform thin film devices, as well as the results with the thin film CIGS sample of the previous section. For this reason the OMP algorithm was also used for reconstruction, as in the case of the simulations in chapter 4 it provided slightly better results for very uniformly performing devices. The reconstructed current maps using the OMP algorithm for this sample are presented in Figure 5.18. Visually, the difference is marginal. The OMP algorithm seems to initially converge faster to the true current map and pixelation effects seem to be slightly reduced, especially when using fewer measurements (<50\%). Nevertheless, the same number of measurements needs to be acquired for detecting the small sharp spots that are obvious in the point by point scan. For a more quantitative comparison of the performance of each algorithm, the correlation coefficient between the reconstructed current maps and the raster scan was calculated. The results are presented in the graph of Figure 5.19. 

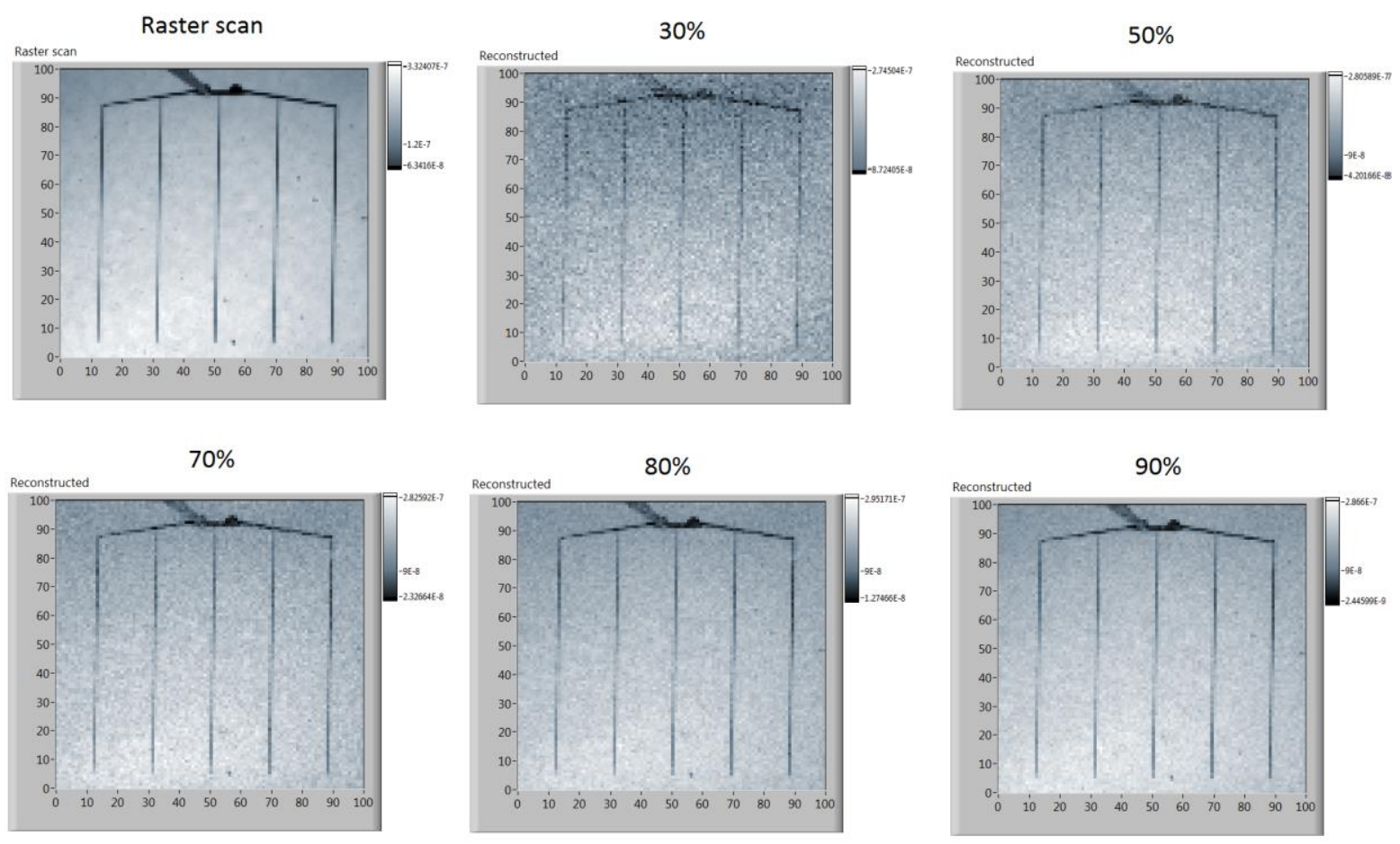

Figure 5.17. Reconstructed current maps of the CIGS PV cell, using the $\ell 1$ minimisation reconstruction algorithm. The percentages indicate the ratio of samples used to the total number of pixels.
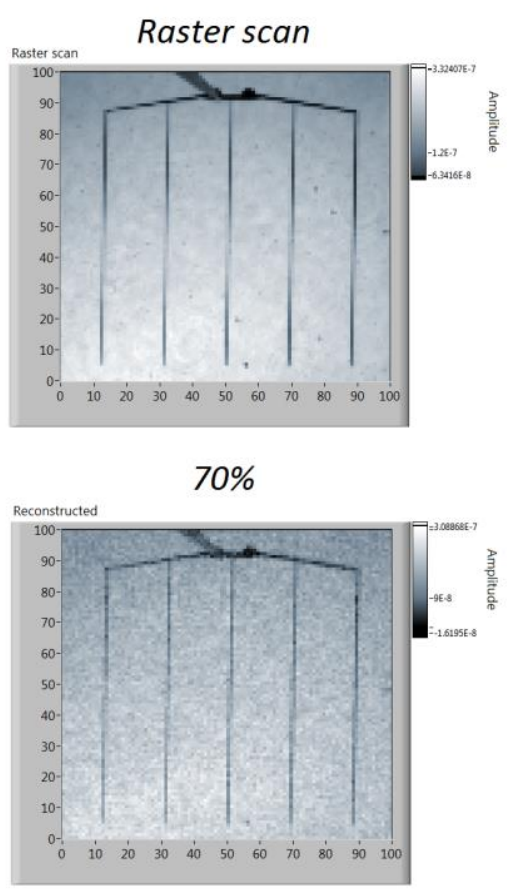

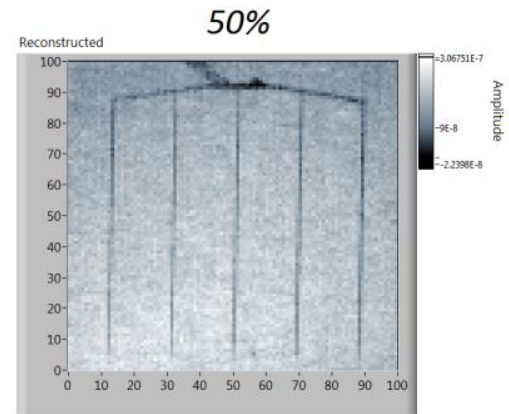

$90 \%$
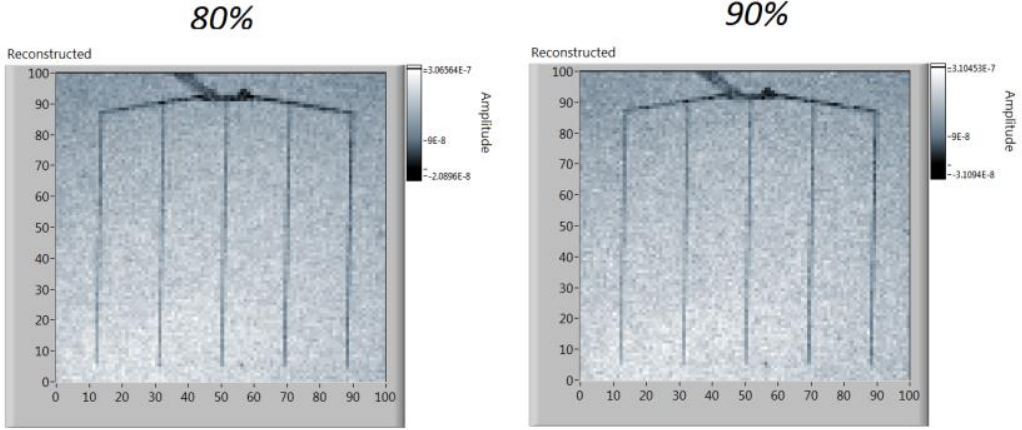

Figure 5.18. Reconstructed current maps of the PV cell, using the OMP reconstruction algorithm. The percentages indicate the ratio of samples used to the total number of pixels. 


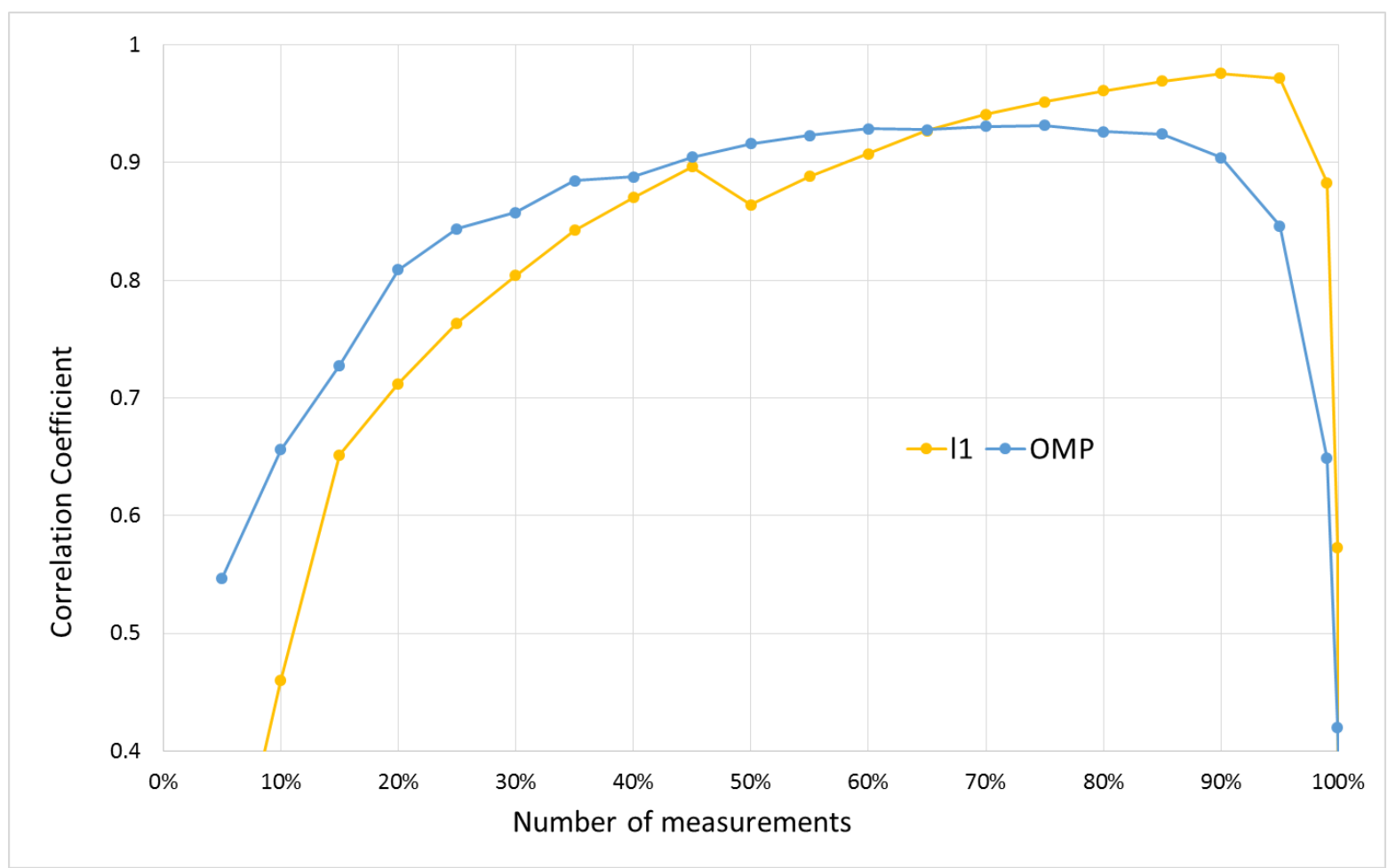

Figure 5.19. Correlation coefficient between the reconstructed current map and the raster scan, as a function of number of measurements acquired, for both the $\mathrm{l} 1$ and the OMP reconstruction algorithms.

From the correlation coefficient graph, the main finding is that the OMP algorithm performs better for up to $65 \%$ of measurements acquired than the $\ell 1$ minimisation algorithm for this sample and this level of resolution. The main reason is the pixelation observed in the $\ell 1$ case for this undersampling range. However, when acquiring over $60 \%$ of measurements, the $\ell 1$ performs better as it seems it is less affected by measurement noise. It should be noted that for this resolution, the OMP algorithm is significantly slower. While the $\ell 1$ needs seconds or a few minutes to reconstruct the current map depending on the number of measurement acquired, when acquiring more than $60 \%$ of measurements the OMP needs up to several hours for large sampling levels, making processing time at least two orders of magnitude longer. This was not completely unexpected as it was also demonstrated in the previous chapter. It is clear that the choice of reconstruction algorithm can have a significant impact for this current mapping method. The step down at $50 \%$ of measurements for the $\ell 1$ is unexpected as it has not occurred in previous results. It could be explained due to the symmetry of the sample and the fact that the discrete cosine transform (DCT) is used, which can be influenced by symmetric structures. This is not a feature of the specific sensing matrix 
used, since it is observed for any sensing matrix applied to this sample when using the $\ell 1$ algorithm, as will be shown later. Such a step is not observed for the OMP algorithm.

Although a point by point current mapping scan could not be realised for the large mcSi sample due to the high amounts of background noise, compressive sampling can still provide reliable results, as also demonstrated already with the previous implementation. Similarly to the previous set of measurements, the same small $1 \mathrm{~cm}$ by $1 \mathrm{~cm}$ area of the cell is measured, that is known to contain cracks and spots. The CS current mapping results are presented in Figure 5.20. A raster scan with our setup results in an extremely noisy current map, which is included in the same figure, where even the sharpest features of the cell area are barely distinguishable. As this is a large area sample, the noise levels are very high, the current signal of the point by point scan is very weak and even with the best amplification the signal is lost within the noise, even though readings are normalised to a reference measurement. No lock-in techniques are used for measurements, which could help reduce measurement noise significantly. In this case, acquiring a current map seems impossible. However, when using compressive sampling, the current signal is greatly enhanced, exceeding the background noise threshold. The reconstructed current maps using the $\ell 1$ minimisation reconstruction algorithm are presented in Figure 5.20.

LBIC maps of the sample using the latest high precision LBIC system at CREST are also presented in the figure, using similar laser wavelengths [107]. Both the CS current mapping and the LBIC scans were implemented with a sampling rate of $10 \mathrm{~S} / \mathrm{s}$. This means, that when CS current mapping is applied, the acquisition of fewer measurements leads to a decrease in measurement time. For producing the CS current maps, both available laser sources were used for measurements, having wavelengths of $658 \mathrm{~nm}$ (red) and $785 \mathrm{~nm}$ (infrared). By using the IR laser, the crack which is obvious in previous EL images and in the $785 \mathrm{~nm}$ LBIC map image becomes visible when increasing the number of measurements. Since the light still does not penetrate deeply into the cell, the crack still appears much thinner than in the EL image. The results are almost identical with the maps of the previous implementation, although illumination uniformity has been slightly improved and there are no misalignment distortions such as the inclination of the grid fingers observed previously. Even though there is some pixelation as a result of the reconstruction process, the reconstructed maps exhibit comparable sharpness and accuracy to the LBIC maps. This is evident especially from $60 \%$ of measurements and above. 
Raster scan with DMD

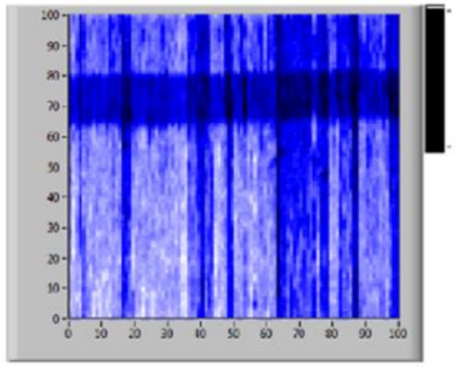

CS $40 \% 658 \mathrm{~nm}$

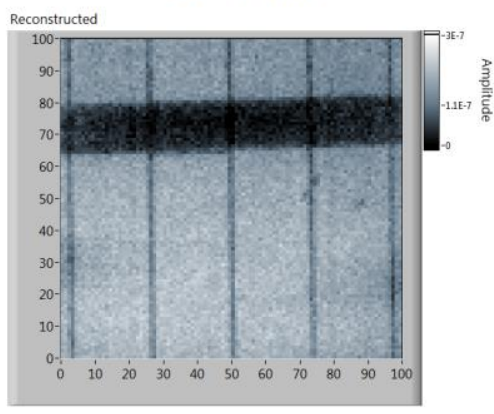

CS $40 \% 785 \mathrm{~nm}$

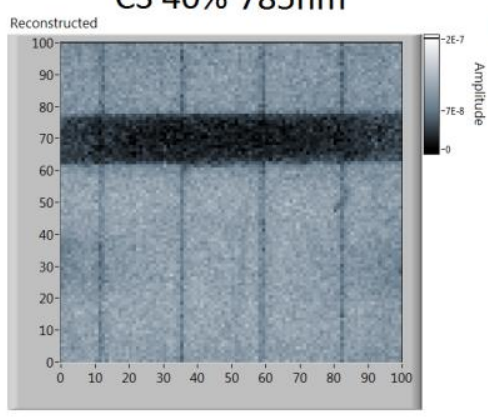

LBIC scan $635 \mathrm{~nm}$

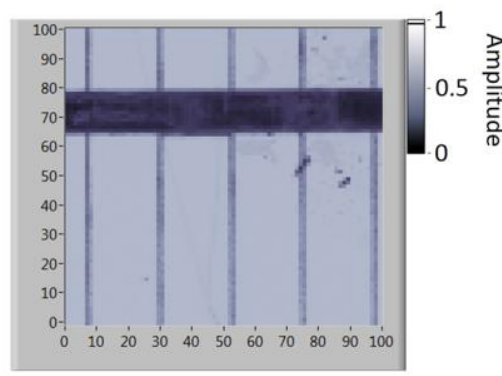

CS $60 \% 658 \mathrm{~nm}$

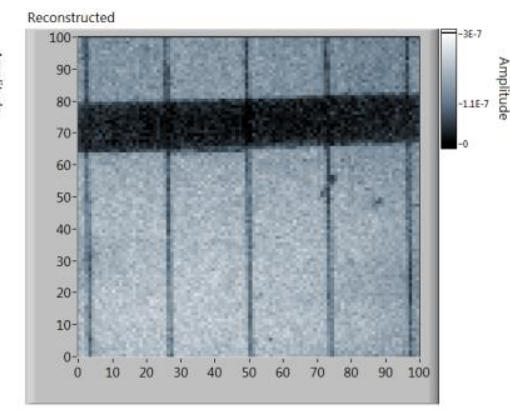

CS $60 \% 785 \mathrm{~nm}$

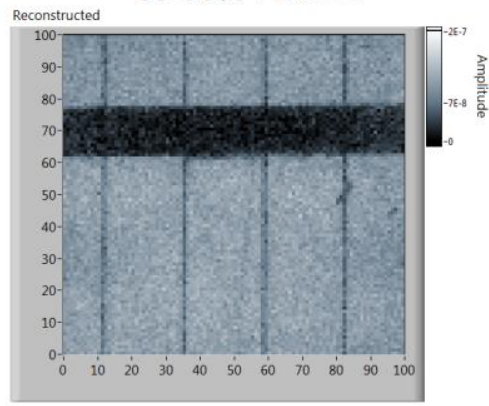

LBIC scan 785nm

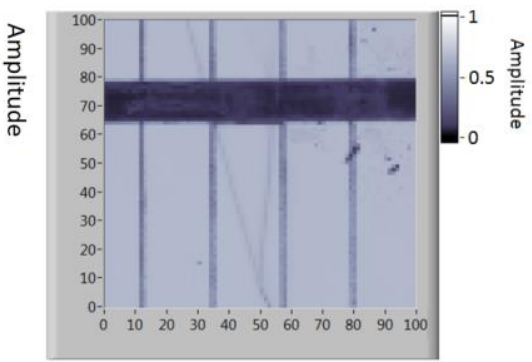

CS $80 \% 658 \mathrm{~nm}$

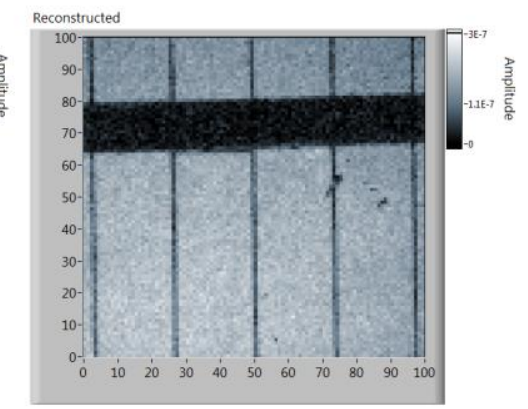

CS $80 \% 785 \mathrm{~nm}$

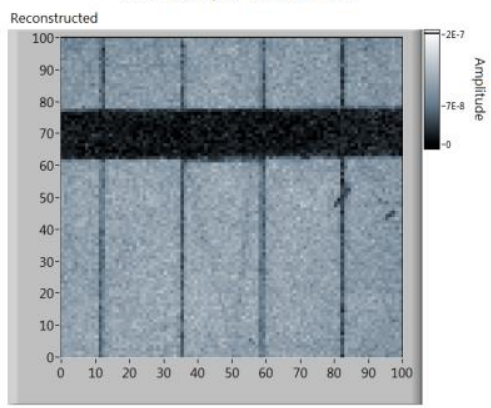

Figure 5.20. In the top row, the noisy raster scan of the small area of the mc-Si cell using the optical system of this work and LBIC scans with the latest system at CREST. In the $2^{\text {nd }}$ and $3^{\text {rd }}$ rows, the reconstructed CS current maps, using the two available laser sources and the $\ell 1$ minimisation reconstruction algorithm. Both the CS current mapping and the LBIC scans were implemented with a sampling rate of $10 \mathrm{~S} / \mathrm{s}$.

In Figure 5.21 the reconstructed current maps using the OMP algorithm are presented. The results are visually almost identical to the ones when the $\ell 1$ minimisation algorithm is used. Similar to the CIGS sample case, the OMP algorithm gives better results when fewer than $60 \%$ of measurements are used, while it has the same performance when acquiring more measurements. Unfortunately, when acquiring more than $60 \%$ of measurements 
reconstruction time becomes very large, in the range of hours, which makes the OMP algorithm impractical for high throughput of measurements with such sampling levels.

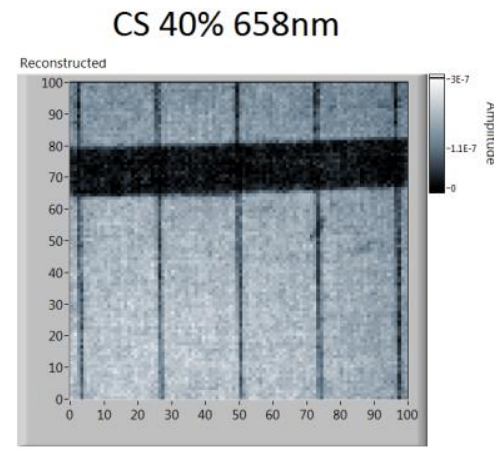

CS $40 \% 785 n m$

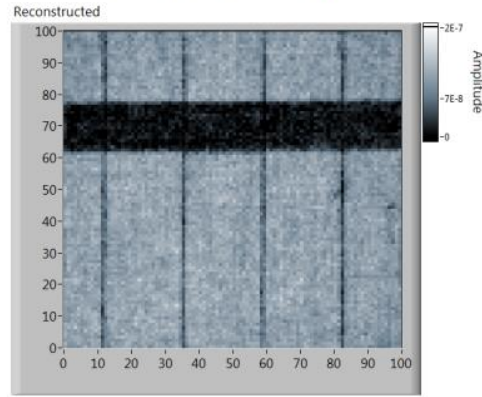

CS $60 \% 658 \mathrm{~nm}$

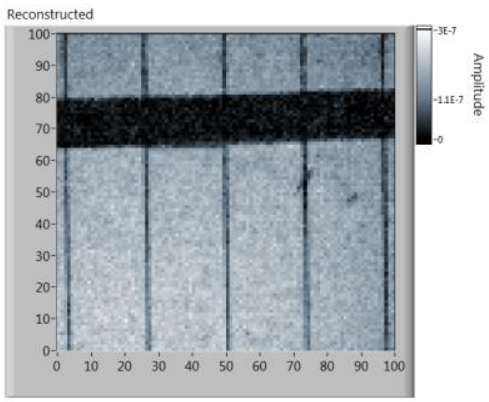

CS $60 \% 785 n m$

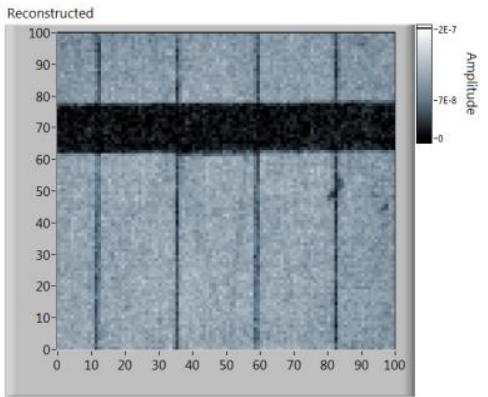

CS $80 \% 658 \mathrm{~nm}$

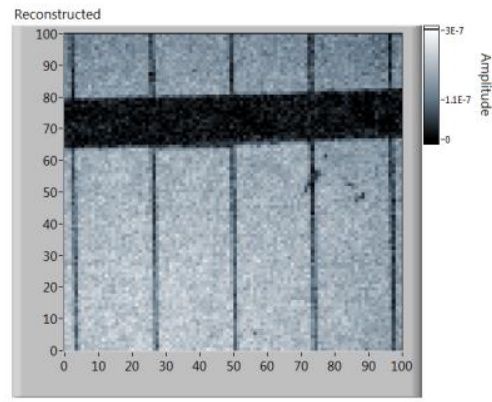

CS $80 \% 785 n m$

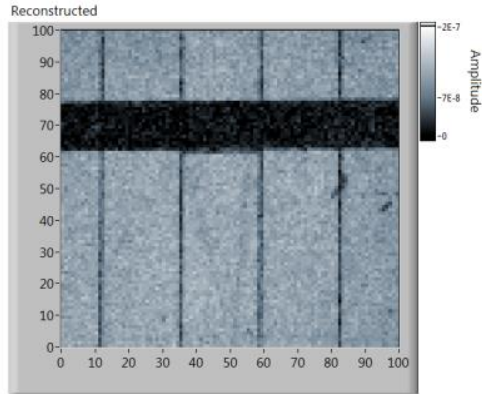

Figure 5.21. The reconstructed CS current maps, using the two available laser sources and the OMP reconstruction algorithm

Direct pixel by pixel comparison of the reconstructed results with a raster scan made with the DMD is not possible for this sample since the raster scan map is extremely noisy and cannot be used as a reference for comparison. Comparing with the accurate LBIC maps would also not yield meaningful results as they were acquired using a different measurement system and a spatially accurate pixel by pixel comparison would not be possible due to image alignment mismatch. Nevertheless, combining the results from both samples it is demonstrated that CS current mapping using this optical setup can be a very practical solution for a simple spatial characterisation instrument. Some further considerations regarding sensing matrices and signal to noise ratio (SNR) are discussed in the following section. 


\subsection{Sensing matrix sparsity}

In this section the impact of sensing matrix sparsity on the measurement process and reconstruction performance is investigated. $100 \times 100$ pixel sensing matrices were used, with different levels of sparsity, having a proportion of pixels in the "on" state from $1 \%$ to $50 \%$. $50 \%$ means that half of the elements of the sensing matrix are one (1) and the rest are zero (0). As a result, the projected patterns on the sample have half of their pixels bright ("on") and the other half dark ("off"). $1 \%$ simply means that only $1 \%$ of the pixels are in the "on" state, which means 100 illuminated pixels for a 10000 pixel projection. This means that the current response measured when a series of patterns (sensing matrix) are projected on the sample will depend on the sparsity of the sensing matrix. As a result, this influences signal levels and so has an impact on the measurement SNR. It should be noted that "measurement SNR" is the SNR at the sampling level; the final SNR of the reconstructed current maps will not be that high and will also depend on the noise inserted by the reconstruction procedure. In reality, initial sampling SNR is only one of the factors that influence the final SNR of the reconstructed image, but it is still a very significant factor for compressive sampling, as will be shown below.

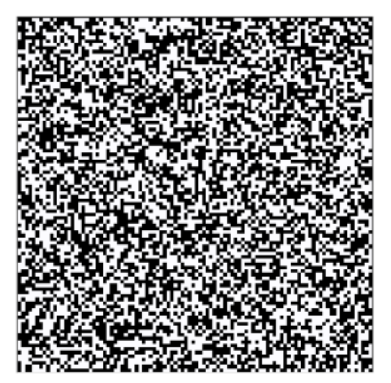

$50 \%$ pixels "on"

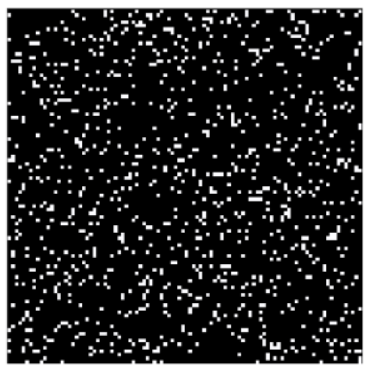

$10 \%$ pixels "on"

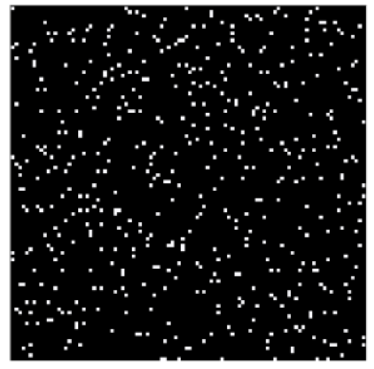

$5 \%$ pixels "on"

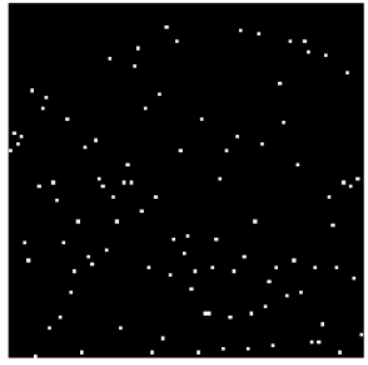

$1 \%$ pixels "on"

Figure 5.22. Visualisation of the sparsity of individual patterns of sensing matrices. Sparser patterns will have many more zeroes than matrices with lower sparsity and this is equivalent to a larger proportion of micromirrors being in the "off" state.

It has already been demonstrated in CS microscopy that sparser sensing matrices have better performance [203]. When using very sparse sensing matrices the probability of having two adjacent pixels at the "on" state at the same time is small. If in a projected pattern there are two adjacent pixels at the "on" state simultaneously, the result may be an overlapping excited area in the sample; In CS application cases as in the optical system of this work, due to light scattering and diffusion of charge carriers, it may be uncertain at which of the two adjacent pixels the additional measured signal that contributes to the global current reading 
of the specific pattern is generated. Consequently, there is eventually increased measurement noise in the final reconstructed current map because of this uncertainty.

These assumptions are confirmed when using sensing matrices with different levels of sparsity for CS current mapping. In Figure 5.23 the 60\% reconstruction current maps are presented for the CIGS cell, using the $\ell 1$ minimisation algorithm for reconstruction. It is visually evident that the reconstructed current maps are becoming sharper when sparser sensing matrices are used. In addition, pixelation effects are significantly reduced with sparser matrices. The same investigation was also realised using the OMP algorithm for reconstruction. The reconstructed current maps are presented in Figure 5.24. The results are very similar to those when using the $\ell 1$ minimisation algorithm; sparser sensing matrices result in sharper images with less pixelation.

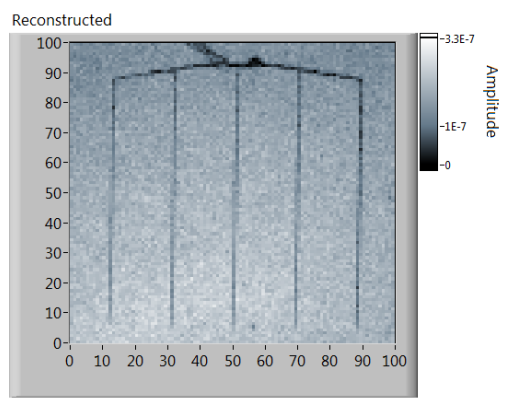

$50 \%$ pixels "on"

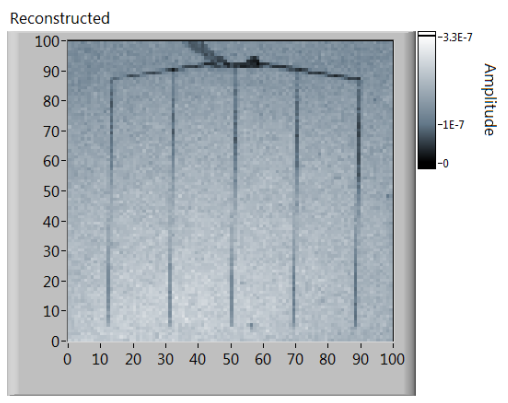

$3 \%$ pixels "on"

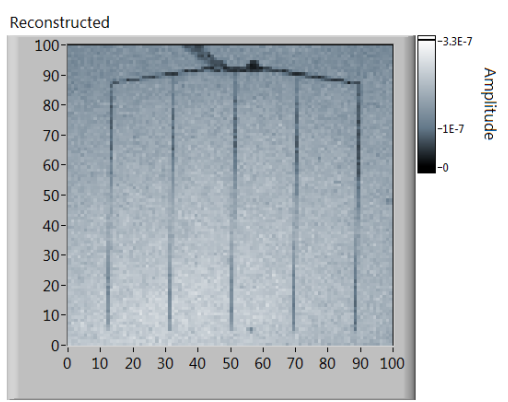

$10 \%$ pixels "on"

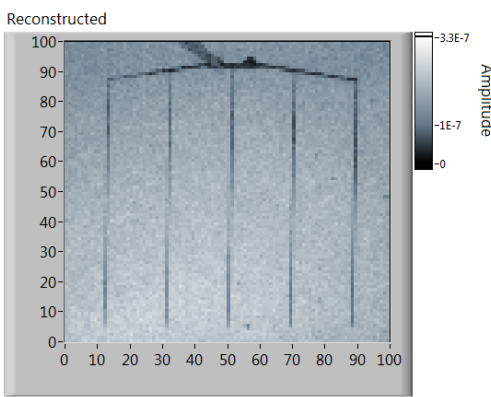

2\% pixels "on"

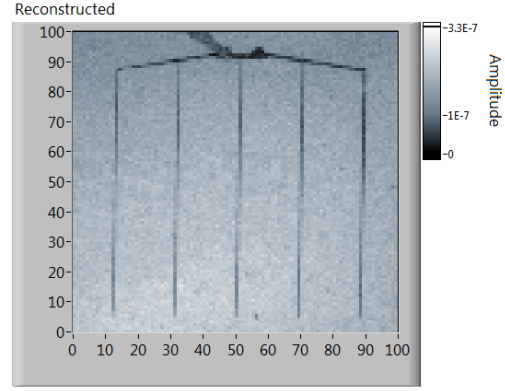

$4 \%$ pixels "on"

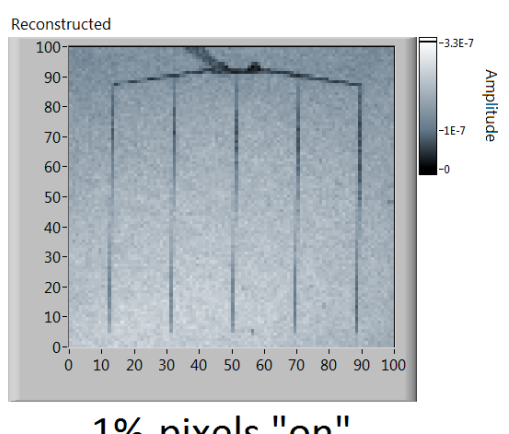

$1 \%$ pixels "on"

Figure 5.23. Reconstructed current maps using $60 \%$ of measurements and the $l 1$ minimisation reconstruction algorithm, acquired with sensing matrices of different sparsity levels.

In order to realise a more meaningful quantitative comparison, the correlation coefficient between the point by point map and the CS current maps was calculated, using obviously the same optical and display resolution for the two methods. The correlation coefficient curves for all sensing matrices and for both algorithms are presented in Figure 5.25. It is evident from the graph that sparser sensing matrices provide better results and 
reconstructed current maps converge faster to the real current map. This general trend is the same regardless of the algorithm used.

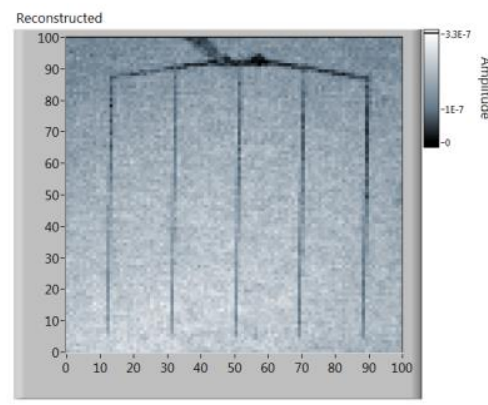

$50 \%$ pixels "on"

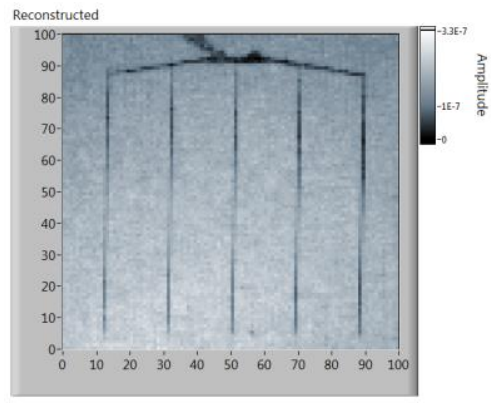

$3 \%$ pixels "on"

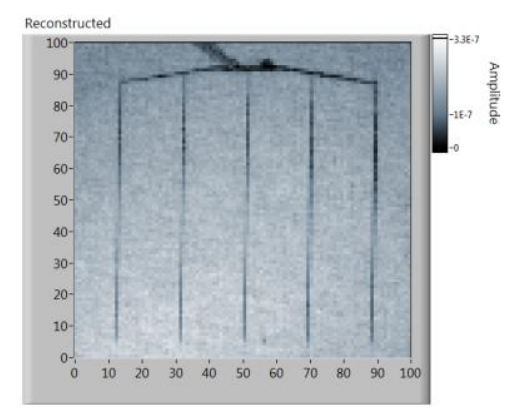

$10 \%$ pixels "on"

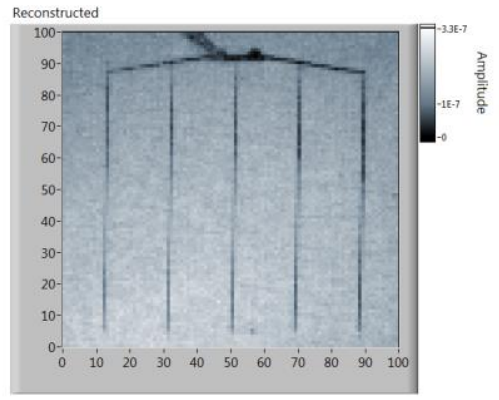

$2 \%$ pixels "on"

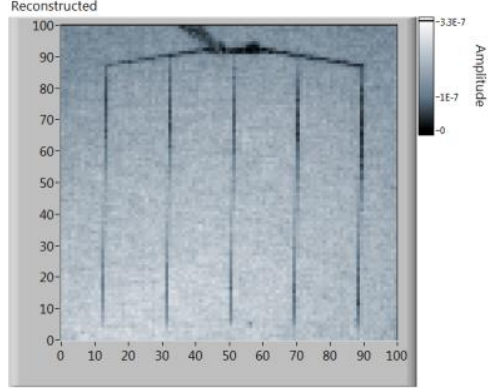

$4 \%$ pixels "on"

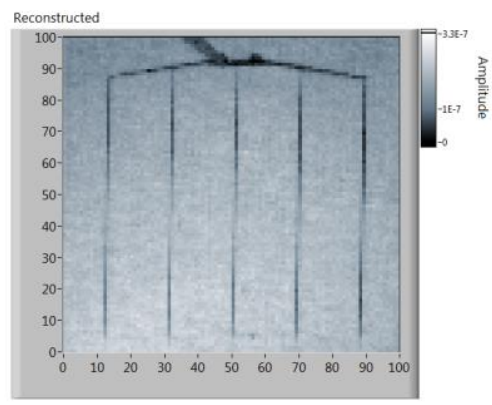

$1 \%$ pixels "on"

Figure 5.24. Reconstructed current maps using $60 \%$ of measurements and the OMP reconstruction algorithm, acquired with sensing matrices of different sparsity levels.

The small local decrease observed in the curves at $50 \%$ of measurements is present for all sensing matrices when using the $\ell 1$ minimisation algorithm. This shows that this behaviour is mainly due to the specific features of the sample that influence the reconstruction process with the specific algorithm, as discussed previously. For this algorithm, the improvement due to sparser matrices becomes clear when acquiring more than $60 \%$ of measurements. There is a great improvement from $50 \%$ to $10 \%$ of pixels at the "on" state, but using even sparser matrices has a very small effect on reconstruction performance. When using matrices of 3\% pixels "on" and above the performance improvement saturates. Similar to the results of the previous section, the OMP algorithm converges faster, although when acquiring more than $60 \%$ of measurements the reconstruction time becomes very large which makes its application impractical. The improvement is significant when comparing the $50 \%$ with the $10 \%$ pixels "on" sensing matrices, but for sparser matrices there is almost no difference in performance, similar to the $\ell 1$ reconstruction case. This behaviour can be crucial in CS applications, since using sparser sensing matrices means that the signal levels are decreasing. 
In this series of experiments where 10000 pixel sensing matrices are used, $10 \%$ means that 1000 pixels are still at the "on" state, which results in a signal amplification of 1000 times, compared to the point by point scan. Nevertheless, even this amplification may not be enough in cases of high background noise levels and this is demonstrated when compressive sampling with different sparsity sensing matrices is applied to the mc-Si sample. Another interesting result when using sparse sensing matrices is that the reconstruction for $100 \%$ measurement acquisition does not fail for the OMP algorithm.

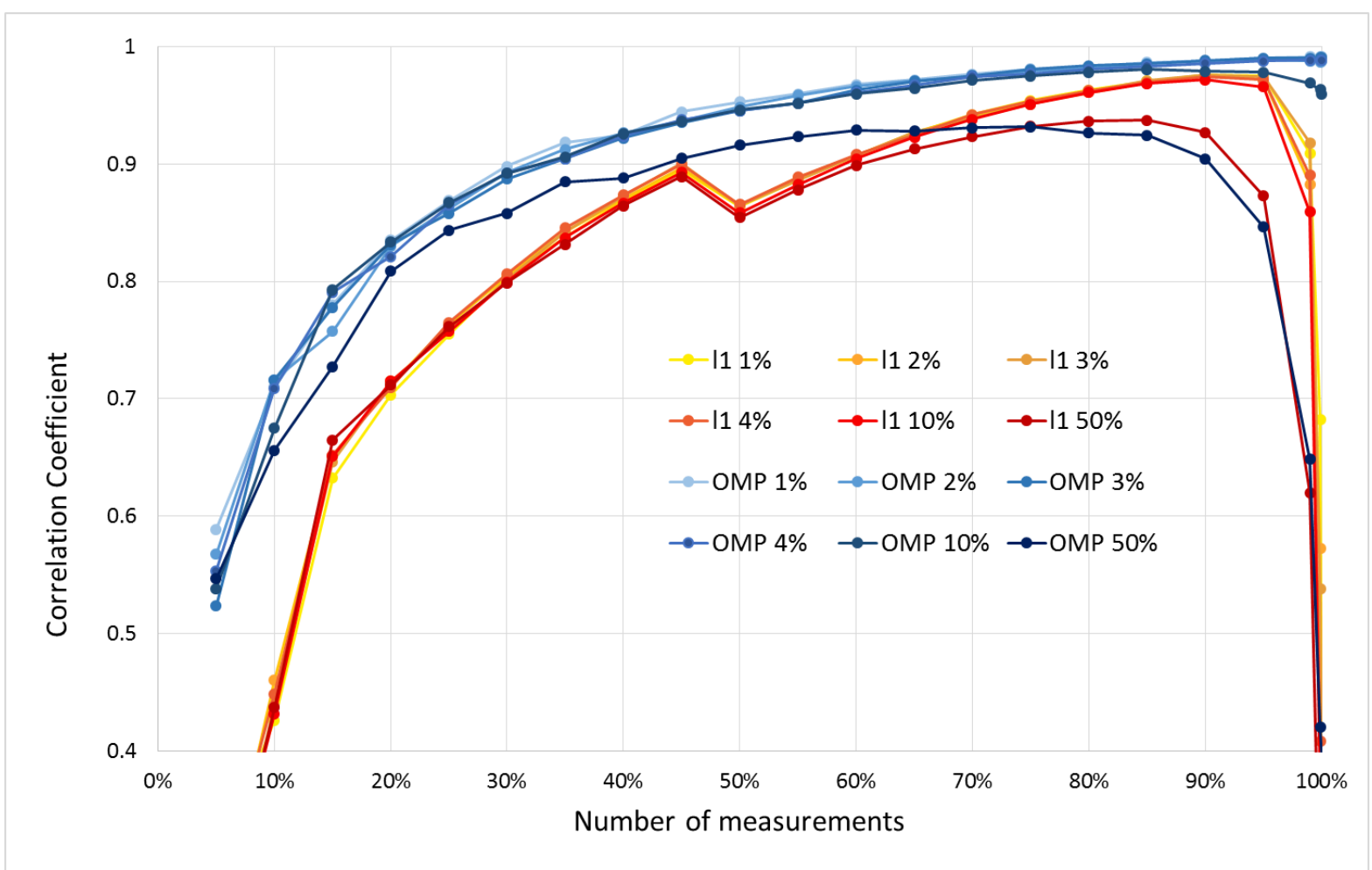

Figure 5.25. The correlation coefficient between the CS current maps and the LBIC map, as a function of number of measurements acquired, for sensing matrices of different levels of sparsity. Results of both the $\ell 1$ minimisation and the OMP reconstruction algorithms are included.

In the case of the mc-Si sample, sensing matrices of $50 \%$ pixels "on" work better than sparser matrices as the measured signal is significantly increased, providing more accurate current readings far above the noise threshold for this sample. Matrices of $1 \%$ pixels "on" do not provide any results at all, as the current signal levels are comparable with the point by point scan. Reducing sensing matrix sparsity gives better and better reconstruction results, as can be seen in Figure 5.26, for $70 \%$ of acquired samples for reconstruction and using both reconstruction algorithms. It is clear from these results that the most suitable sensing matrix 
sparsity depends on the sample and the measurement noise levels of the particular application. Although this means there may be a compromise between accuracy and signal amplification when using CS current mapping, it shows that this technique can be very versatile; current maps of a sample can be acquired even with high background measurement noise.

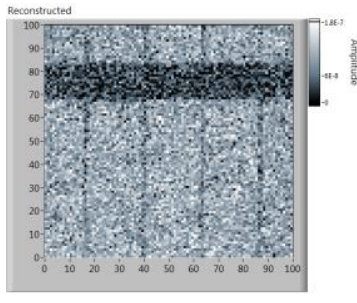

I1 5\% pixels "on"

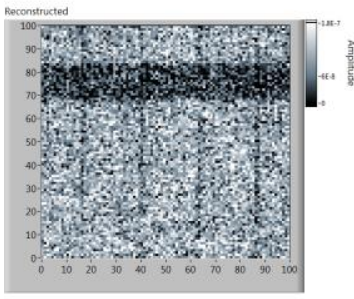

OMP 5\% pixels "on"

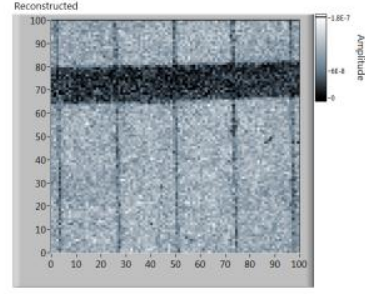

I1 10\% pixels "on"

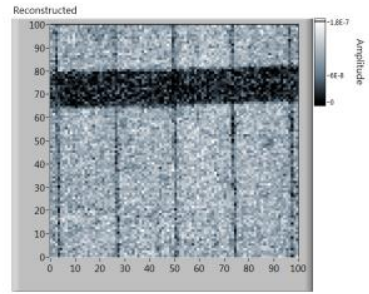

OMP $10 \%$ pixels "on"

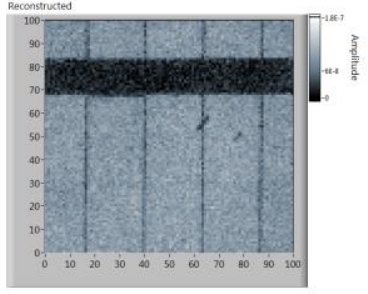

I1 20\% pixels "on"

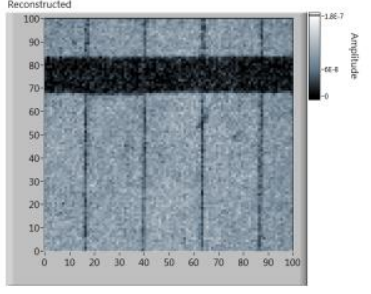

OMP $20 \%$ pixels "on"

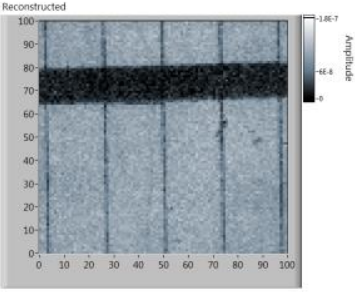

I1 50\% pixels "on"

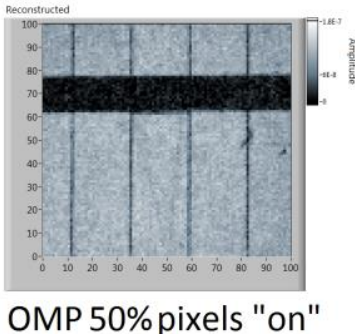

Figure 5.26. CS reconstructed current maps for the mc-Si sample, using $70 \%$ measurement acquisition, for different levels of sensing matrix sparsity. Results using both reconstruction algorithms are presented.

In order to show the influence of measurement SNR on the method's performance, SNR was calculated for all cases of samples and sensing matrix sparsity. The results are presented in Figure 5.27. For the sake of completeness, it is noted that SNR is calculated with the formula:

$$
\text { SNR (Signal to Noise Ratio) }=\frac{\text { Mean Value }}{\text { Standard Deviation }}
$$

As mentioned, each current measurement is the mean of 30 readings, for which the standard deviation $(S D)$ is also calculated. For the point by point scans, the average $S D$ of measurements gives approximately $S N R=626$ for the CIGS sample and $S N R=2$ for the large mc-Si sample. As can be seen from the graph of Figure 5.27, when applying compressive sampling the measurement SNR increases significantly in all cases. When increasing sensing matrix sparsity (reducing pixels in the "on" state) though, the SNR for the CIGS sample stays 
constant, while for the mc-Si sample it decreases, which has an impact on reconstruction quality. This is reflected in the current maps of Figure 5.26. These results show that high SNR is important for CS measurements as when it decreases below 4000 it is evident that the reconstructed current maps are very noisy.

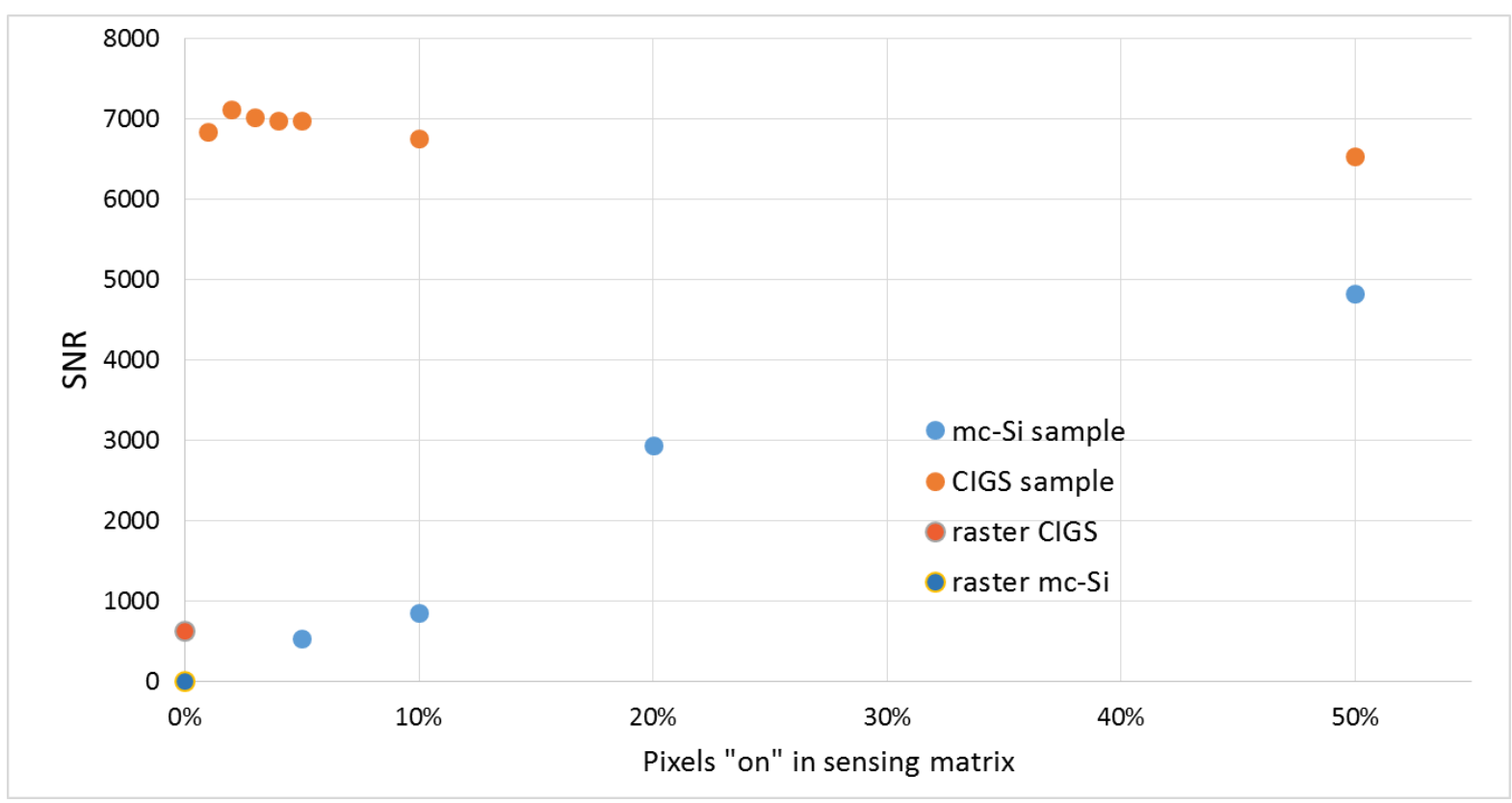

Figure 5.27. Measurement SNR when compressive sampling is applied, as a function of sensing matrix sparsity.

The above results show that the choice of sensing matrix sparsity is a compromise between accuracy and measurement SNR levels. When background noise is low, a $10 \%$ sparsity is optimum, as there is no practical advantage of using even sparser matrices. With increasing background noise, sparsity should be reduced, so that the absolute signal levels are increased. It can be concluded from the graph that CS current mapping requires a measurement SNR of at least 4000 for reliable reconstruction results. In reality, it would not be practical to measure such large samples as the mc-Si with this optical setup; it is designed for small area PV devices as its measurement area is limited to $1 \mathrm{~cm}$ by $1 \mathrm{~cm}$. Consequently, such high noise levels are unlikely. Nevertheless, research PV samples can have very low efficiency so such high relative noise levels can still occur. 


\subsection{Practical Considerations and Discussion}

Some aspects of using such a simple experimental setup for current mapping have to be considered. Regarding the optical setup, it is mentioned already that the beam uniformity after the DMD is not absolutely perfect. This minor non-uniformity can be remedied by inserting additional optical elements after beam collimation; an engineered diffuser and an additional spatial filter, to properly re-collimate the beam after the diffuser. Another option would be initial calibration of beam non-uniformities using a CCD camera, although this will not be sufficient for measurements of non-linear PV samples. Since the beam is expanded and only a small portion of the initial laser output is used on the sample, the light losses are significant and high power laser sources are needed. For the $1 \mathrm{~cm}$ by $1 \mathrm{~cm}$ area in our case a $100 \mathrm{~mW}$ laser source is sufficient. The calculated losses are around $70 \%$, which is a significant but inevitable loss. Bias light could be added to overcome this challenge, as well as series resistance effects.

Expanding the measurement area is realistic and will require only a small number of additional optical elements after the generation of the patterns. Additional laser power will be necessary, depending on the size of the measurement area increase. On the other hand, research PV samples are usually smaller than $1 \mathrm{~cm}$ by $1 \mathrm{~cm}$, which means the experimental setup built in this work, which is straightforward to realise, can provide a reliable current mapping instrument for these uses.

A reference measurement of temporal laser intensity is necessary to avoid any instability influencing measurements. This reference measurement is achieved by adding a beamsplitter and a photodiode in the light path. However, the use of a DMD chip for realizing measurements adds a more cost effective and simple way, just by sacrificing half of the measurement speed, when time is not a significant issue. A specific area of the PV cell under measurement, multiple areas simultaneously or even the whole sample can be used between measurements to obtain a reference reading. The same area has to be used throughout measurements so that the reference current reading depends on only laser output. This keeps the measurement setup still extremely simple without additional parts, while the reference measurement is just a matter of software programming. Unfortunately, this concept will only be able to detect long term laser instability and will miss any spikes. Thus, when it is possible a simultaneous reference measurement with another detector is optimum.

The results with the small CIGS PV cell indicate that very high resolution current mapping can be achieved by using the DMD to apply a point by point scan. CS current mapping also provides reliable results, when around $50 \%$ of measurements and above are acquired. As 
was shown in the previous chapter, CS current mapping based on the $\ell 1$ norm minimization algorithm for reconstruction exhibits reduced performance for very uniform samples. In this case other reconstruction algorithms can perform better, such as the Orthogonal Matching Pursuit approach [180]. On the other hand, even if more samples are needed in some cases for reconstructing the current map, compressive sampling always requires fewer measurements than a point by point scan. After acquiring the measurements, the reconstruction process requires approximately a minute for around $50 \%$ undersampling. This is an offline process, which means another scan can start while the previous set is reconstructing.

The sampling rate achieved for the CS current mapping system of this work is $10 \mathrm{~S} / \mathrm{s}$. Such a sampling rate is comparable with conventional LBIC systems in the literature but still slightly low, when compared to state of the art LBIC systems, such as on-the-fly LBIC [92]. Such a system would be able to acquire a current map of 10000 pixels in 5 minutes, while a $50 \%$ CS current map is acquired in approximately 8 minutes. Such a speed is also much slower than EL imaging, which can be implemented in seconds. Nevertheless, measurement speed could be boosted by using the internal memory of the DMD module. Latest DMD models have an even larger internal memory, which could make it possible to load the complete sensing matrix (series of patterns) into the DMD before measurements. This will save significant communication time between software, DMD and the measurement instrument, as in this case only a trigger will be used to synchronise measurement acquisition with pattern projection. Only the switching rate of the micromirrors and the response of the sample will then limit measurement time. Thus, potentially a sampling rate of $100 \mathrm{~S} / \mathrm{s}$ can be achieved. In such a case, a current map of 10000 pixels can be acquired in a minute.

The flexibility in the choice of resolution for the implementation of the measurements is an important feature when using a DMD. By grouping different numbers of micromirrors together it was shown that optical resolution can be altered from $96 \mu \mathrm{m}$ to $27 \mu \mathrm{m}$, without any mechanical movement. In a conventional LBIC system this would be achieved by mechanical movement of optical elements, such as changing the position of a microscope objective lens. On the other hand, the level of optical resolution achieved by LBIC systems reported in the literature can be as high as $1 \mu \mathrm{m}$, by using microscopy configurations [99][84]. The optical system of this work has not achieved such a high resolution, although it would be possible by inserting a microscope objective lens before the sample, in order to demagnify the projected patterns. 
An important advantage of CS current mapping is that it can be applied in cases when a point by point scan fails to provide any results. In our case, a large mc-Si sample was used which inserted high noise levels compared to the current signal. CS current mapping gave reliable results where the signal of the point by point scan was lost within the noise. This shows that a simple current mapping setup can work in a cost-effective way, without lock-in equipment but by adjusting the suitable sampling method depending on the sample and the noise levels. In addition, sensing matrix sparsity levels can be adjusted in a way that optimised accuracy is achieved. When the noise levels are low, sparser matrices have better performance. However, when noise levels are high and it is difficult to implement a point by point scan, less sparse sensing matrices are preferable and provide better results by significantly increasing signal levels.

The experimental results show that the choice of reconstruction algorithm can have a significant impact on the performance of the method. In the previous chapter, the PVONA simulations showed that the $\ell 1$ minimisation algorithm has slightly better performance than the OMP algorithm. Nevertheless, the experimental results showed that the algorithms perform similarly and in cases where sparse sensing matrices are used the OMP can give better results. Especially for measurement acquisition below $60 \%$, the OMP algorithm always performs better. When using CS current mapping the goal for undersampling is at least $50 \%$ and the useful range is within $40 \%$ to $60 \%$ of measurements. Thus, within this range the OMP algorithm seems to be the optimum solution. Nonetheless, there are a large number of reconstruction algorithms in the literature and it is possible that better reconstruction algorithms exist for this application.

\subsection{Conclusions}

An experimental layout for current mapping of small area PV devices has been built, based on a DMD chip with the aim of applying and investigating compressive sampling. This current mapping system is realised in a very simple and straightforward way, involving no moving parts, complicated optical elements or lock-in methods to achieve high optical resolution and sampling rate. It also offers the unique opportunity for an experimental pixel by pixel quantitative comparison between point by point and CS current mapping. Two different algorithms and sensing matrices with different levels of sparsity have been 
compared. Both mc-Si silicon and thin film samples were investigated, as in the case of the simulations in the previous chapter. The optical layout presented here is the first reliable compressed sensing current mapping setup for PV characterisation reported in the literature.

The experimental results confirm that CS current mapping yields reliable results with fewer acquired measurements than a point by point scan. It is shown that undersampling of at least $50 \%$ can be achieved with compressive sampling, which can lead to a significant reduction of measurement time. The method has similar overall performance for all types of samples. By using compressive sampling with this specific experimental setup, the measurement SNR is enhanced compared to point by point sampling. This makes CS current mapping necessary in cases of very high noise levels, where the signal to noise ratio $(S N R)$ of the measurement must be enhanced for meaningful sampling above the noise threshold. Sparser sensing matrices exhibit better performance, although less sparse matrices may be required in the presence of noise, to increase the $S N R$. Further optimisation of this system is possible, as there are still some shortcomings to be resolved, as described in the previous section.

A significant improvement of this experimental layout can be achieved by making the setup more compact. In the experimental implementations in this work, lenses with long focal lengths were used for making optical alignment easier. By using optical elements with less than half of this focal length, the setup can become much smaller. This is a further step for a realistic commercial application of this method, as a compact and simple instrument for CS current mapping can be built that will occupy a small amount of space. Using a more compact setup, the cost of building this instrument will also be reduced, due to lower space requirements for the enclosure of the system. This simple DMD current mapping system can be utilized for reliable spatial characterisation not only for PV devices, but also for any type of photodetectors and other semiconductor devices, requiring only selection of a suitable laser source.

The core of this current mapping instrument is the DMD chip, which generates the patterns that are projected on the sample. Consequently, a scale up of this method can be investigated using Digital Light Processing (DLP) projection technology, where DMD modules are used for producing the projection images. Most modern projectors, from small room projectors to high power cinema projectors, now use DLP technology, due to its high contrast ratio and overall performance. An initial investigation of the concept of using a commercial DLP projector for CS current mapping of PV modules is realised in the next chapter. 


\section{Chapter 6}

\section{Scalability of Compressed Sensing Current Mapping}

\subsection{Overview}

Although current mapping is important for PV characterisation, applying LBIC measurements to full-size PV modules presents some practical difficulties. Bias light is necessary in all cases, since if all cells are in the dark and only one is illuminated, no current will be measured at the contacts of the module. Additional shading strategies on the cell under measurement then also need to be adopted in order to force its role as the currentlimiting cell and acquire current maps of PV modules of interconnected cells [78]. Finally, the time needed to conduct a point by point scan on a large module is prohibitive for practical applications at useful resolutions. It is thus difficult to measure the spatial current response of large PV modules and as a result other methods are preferred such as EL imaging or lockin thermography.

In the previous chapters, CS current mapping was investigated through electrical simulations as well as with a small area optical measurement setup. In chapter 4, apart from the simulations for confirming this measurement method, the concept of CS current mapping of series interconnected PV modules was investigated. It was shown that in principle this is possible, as in the absence of bypass diodes the current output from a PV module will be 
limited by the cell that produces the least current. As was demonstrated, when the patterns for compressive sampling are projected on an individual cell of the PV module while the rest of the cells are fully illuminated, the current measured at the contacts of the module will be dominated by the current produced by the cell under measurement. This will stand provided that the other cells each produce more current than the cell under test. This feature of CS current mapping implementation, along with the fewer measurements required, has the potential to make current mapping of even large, multi-cell PV modules possible. A first approach of this concept is investigated in this chapter.

CS current mapping has already been successfully applied to small area devices, as it was described in the previous chapter. The sampling patterns were generated by a DMD module for the realisation of compressive sampling. Reliable current maps have been produced by acquiring just $40 \%$ of the measurements that a standard LBIC system would need, with a sampling speed of 10 samples per second achieved. In this chapter, a digital light processing (DLP) projector is used to replace the laser sources of the small-area setups. In DLP projectors, the image is created by DMD chips, where each mirror represents one pixel in the projected image. They are functionally the same as the DMD described in the previous chapters and for this reason, a DLP projector can theoretically be used for applying the necessary illumination patterns for CS current mapping of large PV devices. Such an approach may provide a cost effective way of scaling up the CS current mapping technique for spatial characterisation of PV modules. Point by point current mapping using a DLP projector has already been proposed in the literature [166]. It has also been shown that by utilising projection technology, one can achieve high accuracy and robustness in projecting any structured patterns for spatial characterisation measurements [204].

The aim of this chapter is to demonstrate that a DLP projection based system for CS current mapping can be a feasible solution for PV module characterisation. Such a system would provide a convenient means to project the required patterns onto the sample under measurement, in order to apply compressive sampling. This can be achieved by projecting patterns on the cell under measurement while light biasing the rest of the cells at the same time, following the principles detailed in [78]. In the following, a standard commercial DLP projector is used in an experimental layout, to investigate the issues and features of this approach. The feasibility of CS current mapping with a DLP projector, for both single PV cells and PV modules is investigated and presented in this chapter. 


\subsection{Operating principles of a Digital Light Processing (DLP) projector}

Any display device that uses a DMD module for creating the projections is called a "Digital Light Processing (DLP)" device. DLP technology is used in the majority of modern projection equipment, from small business projectors to cinema projectors. The general principles of a commercial small scale DLP projector with a single DMD chip are described in Figure 6.1. The light from the source, which is usually a broad-spectrum lamp, passes through a colour wheel that separates the different colours. The colour wheel is synchronized with the DMD and filters the light into red, green and blue components. Each micromirror of the DMD is synchronised with these basic colours. By combining these colour components, a single chip DLP projection system can create approximately 16.7 million colours.

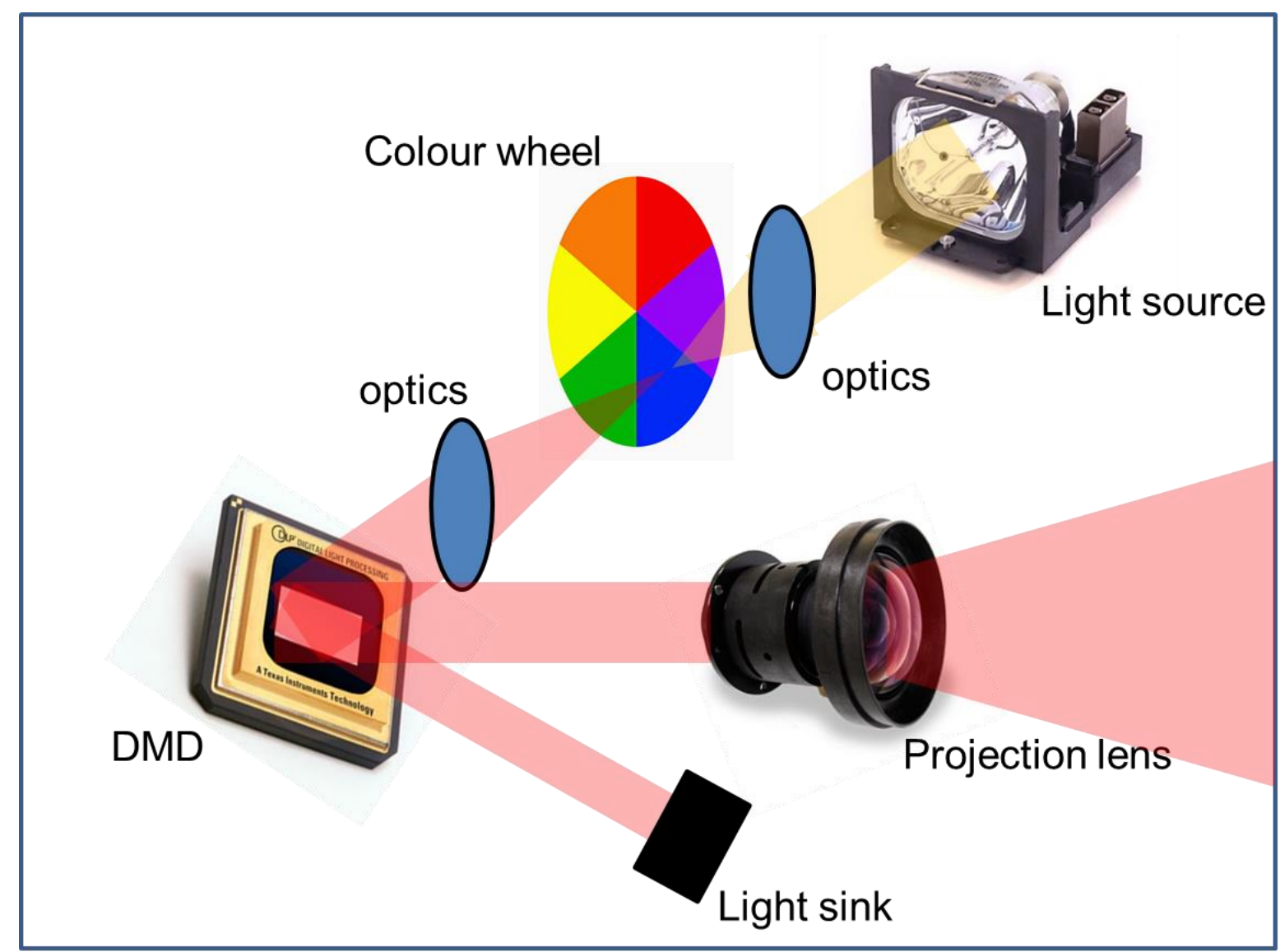

Figure 6.1 Breakdown of a typical single DMD chip DLP projection system

At the projection screen, each illuminated pixel is red, green or blue at any given moment. The DLP technology utilises the perception of a viewer's eyes to blend the pixels into the desired colours of a projected image. As an example, a micro mirror that creates a purple pixel will only reflect the red and blue light to the projection screen. The pixel on the screen is actually a rapidly alternating flash of blue and red light. The balance of blue or red 
components over time determines the final shade of purple that the eyes of the viewer will perceive. The projection lens magnifies the image that the DMD creates, onto the projection screen. A light sink is also used so that the light that the pixels reject when they are at the "off" state is absorbed efficiently. This ensures that there is no stray light to distort the projection. In the latest models of DLP projectors, colour wheels include more colours, such as yellow, white, magenta and cyan, for even more colour combinations and greater colour rendering accuracy.

DLP cinema projection systems usually consist of three DMD chips. Each one of them has its own dedicated light source: red, green or blue. This can be achieved by passing the white light generated from a single broad source lamp through a prism that divides it into red, green and blue components. Other options that have been demonstrated are separate colour LEDs or laser sources [205][206]. High power DLP projector products have been developed lately utilising laser sources [207].

\subsection{Evaluation of the DLP projector as a light source for current mapping}

\subsubsection{Projector features and initial tests}

The projector used for this work is a typical commercial type, the Acer P7605 DLP projector with a $370 \mathrm{~W}$ metal halide lamp. It is a reasonably high intensity projector, capable of generating a brightness of 5000 ANSI lumens. An important advantage of DLP projection technology is the high contrast ratio that can be achieved. This allows the system to efficiently generate a black pixel equivalent to a masked shaded spot which is significant when projecting patterns of dark and bright pixels. The projector is set on a bench and the illuminated samples are mounted vertically. This setup is not on an optical table so vibrations may affect the spatial accuracy of measurements, although their influence will be minor since the resolution achieved with the projector setup is in the $\mathrm{mm}$ scale. All experiments have been conducted in a dark room, so light contamination from external sources is negligible.

Some initial tests were undertaken in order to analyse the projector output and identify potential challenges. An encapsulated $8 \mathrm{~cm}$ by $8 \mathrm{~cm}$ mc-Si PV sample is used for these initial tests. The sample is identical with the one used in the previous chapter. A constant pattern of black and white pixels is projected onto the sample, similar to the patterns applied for compressive sampling. A photograph of this is presented on the left of Figure 6.2. A Keithley 
2420 is used for voltage bias and the current response is measured with a NI DAQ card. The voltage bias is set at a constant $\mathrm{OV}$. A shunt resistor is used for measuring the current response of the PV device with the DAQ card. The circuit configuration is presented on the right of Figure 6.2.
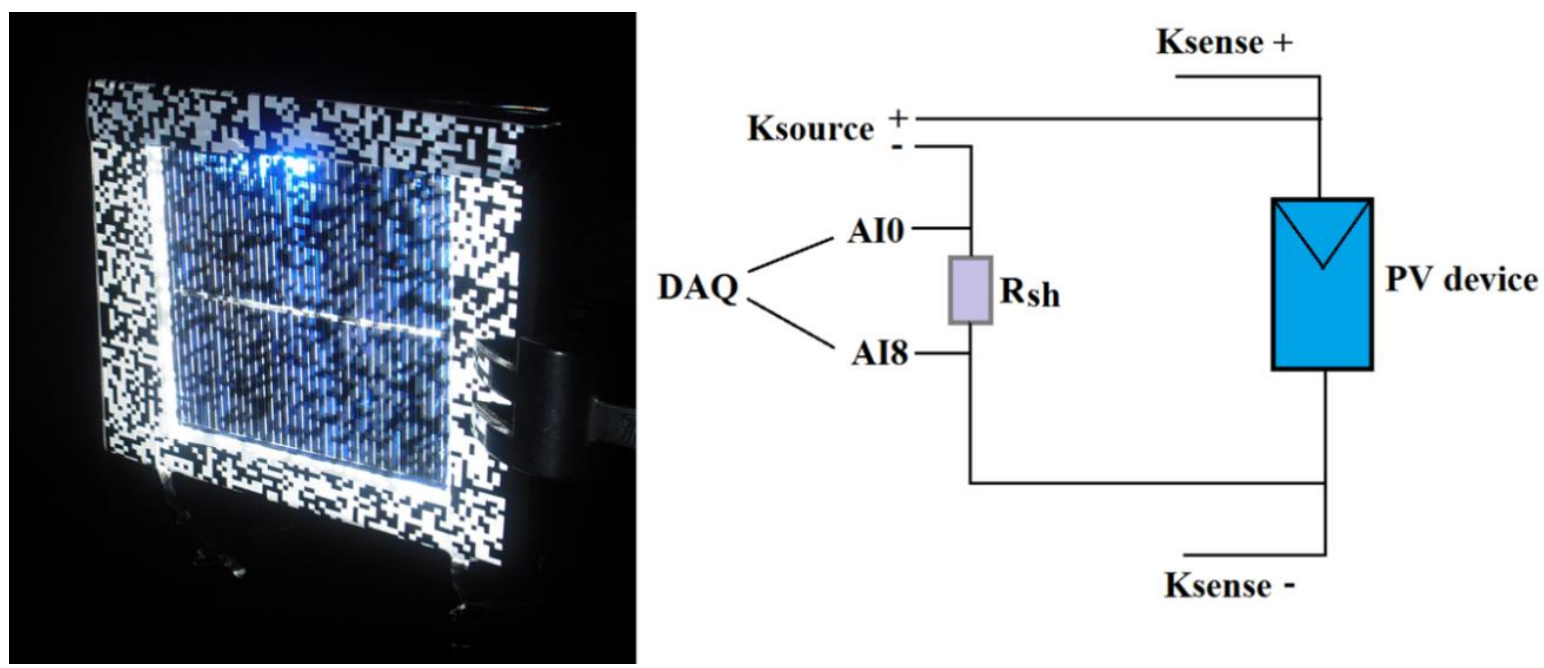

Figure 6.2. On the left, the sample used for initial tests of the projector system, illuminated with a random black and white pixel pattern. On the right, the circuit diagram for making measurements.

Considering the working principles of the DLP projector, temporal, spectral and spatial variations of light intensity are expected. A sampling speed of 250000 samples per second was set for the current reading for a first evaluation of the photocurrent signal. The measured current for 12000 samples $(48 \mathrm{~ms}$ ) is presented on the left of Figure 6.3. It is clear that the light intensity is very unstable, apparently due to both spectral variations from the colour wheel and the uncontrollable switching of the micromirrors of the DMD. In the small area optical setup of chapter 5 there was direct control of the DMD. In this system where the DLP projector is used, the DMD is controlled by the projector's internal system. Although the patterns are created and sent for projection, there is no direct control of the internal DMD of the projector. Even when projecting black and white light patterns, the DMD is still flickering uncontrollably for colour mixing reasons. These variations are periodic; with the $250 \mathrm{kHz}$ sampling rate used, a period is approximately 4170 samples, corresponding to around $16 \mathrm{~ms}$. The signal measured during a whole period is presented on the right of Figure 6.3. Such a light source is highly unstable for current mapping measurements as it is and this will be demonstrated below. 

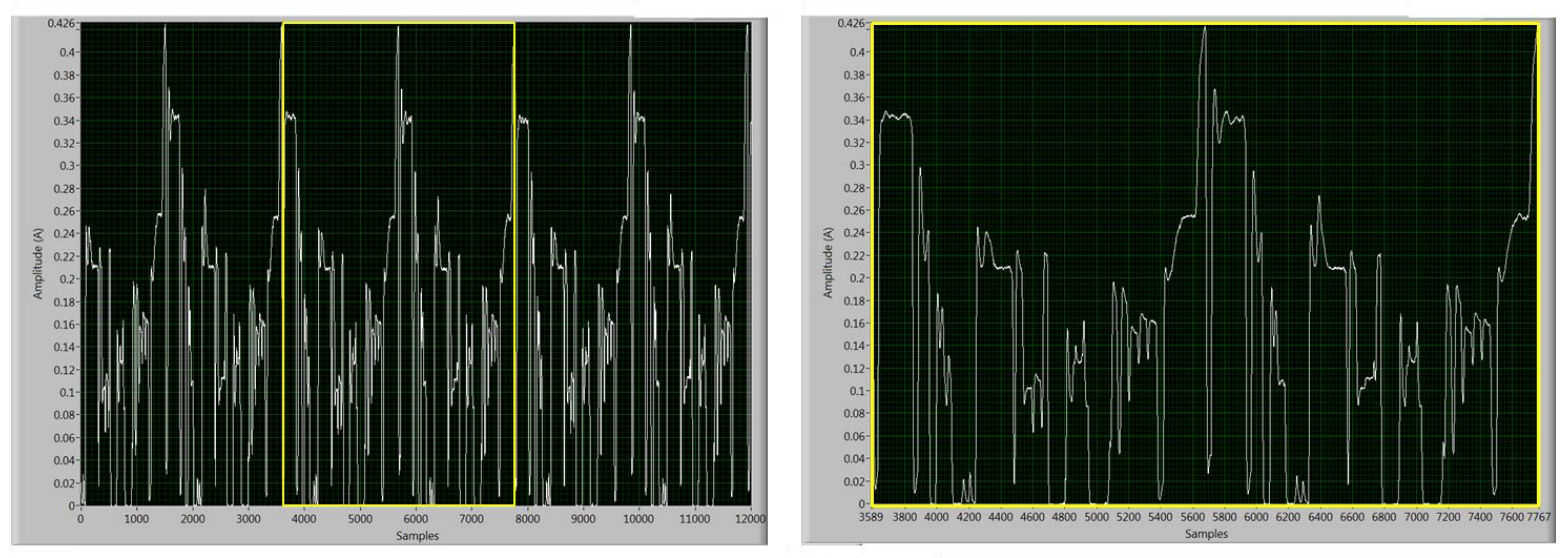

Figure 6.3. Periodic short term temporal variations of the light intensity from the projector. $A$ period is highlighted with the yellow square on the left graph and it is presented zoomed-in on the right.

In addition to these short term variations of light intensity, long term variations have also been detected. In order to evaluate these variations, current measurements need to be stabilised for the short term variations. For this reason, integration over 1 period is realised (4170 readings are acquired and averaged to make one sample reading). This procedure is done 10 times for each current measurement so that the measurement signal is as accurate as possible for a specific projection. Inevitably, this makes the measurement procedure very slow, but is necessary due to the instability of the light intensity. The resulting sampling rate from this strategy is around 2 samples per second. The same pattern is projected on the sample for a long period of time and the current is measured 2000 times with the aforementioned procedure. This corresponds to a total measurement time of approximately 20 minutes. The result is presented in Figure 6.4. It is clear that there are long term fluctuations of light intensity, in the range of $\pm 3.5 \%$ of the average current value. This is not a large amount but it can significantly influence compressive sampled readings and insert background noise in the reconstruction procedure. For this reason a reference measurement needs to be established so that all measurements are normalised to account for these long term fluctuations. This is achieved by adding a photodiode next to the sample, assuring that the projection always has a stable part at the edge (i.e. no pattern variation applied at this part of the projected image, just white light) for monitoring light intensity. Readings from the photodiode are acquired simultaneously with the PV device current measurements. The current signals are then normalised according to any light intensity changes. 


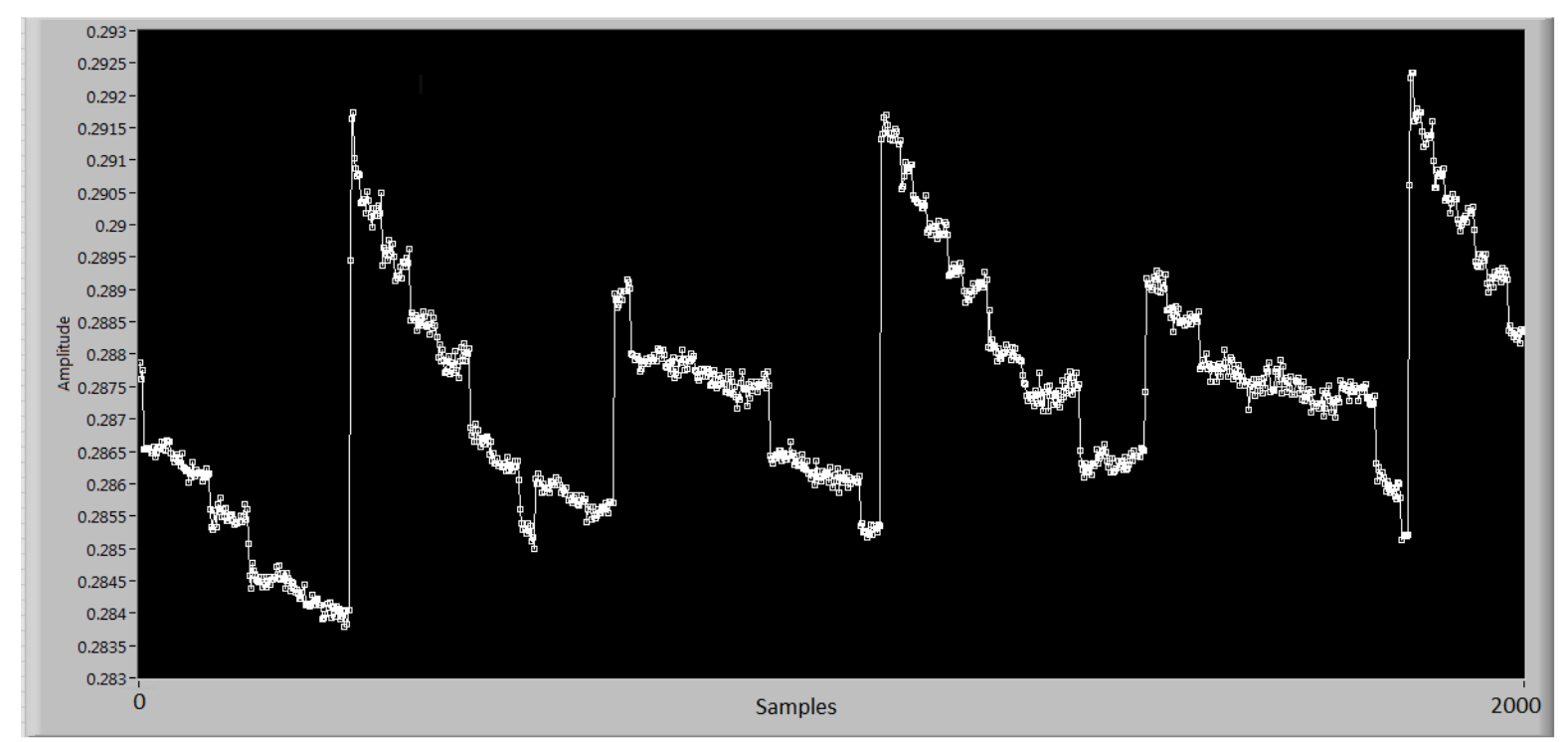

Figure 6.4. Long term variations of light intensity over a period of 20 minutes, acquired by projecting the same pattern on a PV device. The range of the variation is $\pm 3.5 \%$ of the average current measurement value.

Another factor that is expected to influence measurements is the non-uniformity of irradiance on the projection plane. Due to expansion of the beam by the projection lens and additional lens effects, the irradiance is expected to be slightly higher at the centre and lower at the edges of the projection. The homogeneity of the light field generated by the projector was thus investigated and quantified. After placing the projector at a fixed position, the irradiance intensity map of the projection was acquired, using a photodiode on an $x-y$ table. This was placed at a fixed plane parallel to the sample as illustrated in Figure 6.5 (left). The irradiance map is presented on the right of Figure 6.5. The maximum difference in irradiance (maximum - minimum) is approximately $20 \%$ of the average value. However, even when measuring PV modules in the implementation here, the edges of the projection are not used, only the central part. This brings non-uniformity to a value of approximately $10 \%$.

Considering the above inherent issues of the DLP projector when using it as a light source without any modifications, initial CS current mapping was attempted in order to test this concept. A cell identical to the mc-Si cell of the previous chapter is used. The sequence of random patterns for compressive sampling is projected on the sample and the current map is reconstructed using the $\ell 1$ minimisation algorithm as in the previous chapters. 60 by 60 pixel patterns were used by binning DMD physical pixels together, as in the case of the DMD optical setup. The resulting low resolution current map for $50 \%$ measurements is presented in Figure 6.6, along with the EL image of the same sample. Although the method seems to 
work in theory, as a current map is acquired, it is apparent that the noise levels are extremely high and the busbar of the sample is the only distinguishable feature. The main source of this noise is the severe short term instability of illumination, as already presented in Figure 6.3. For this reason, some modifications were made to the DLP projector in order to reduce measurement noise and improve accuracy.
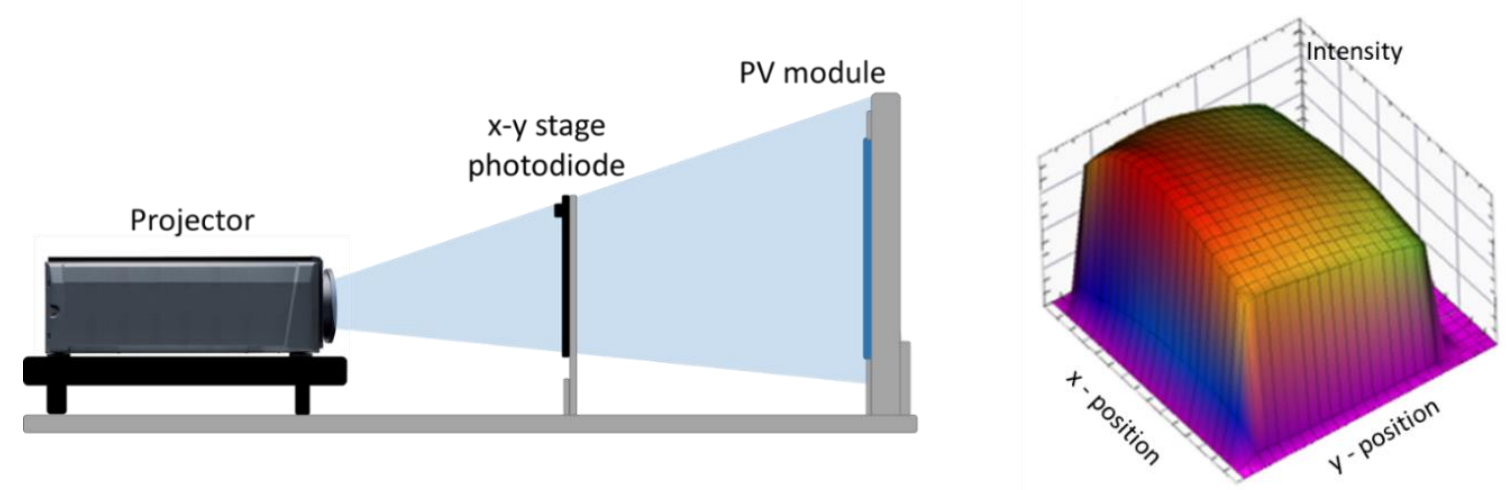

Figure 6.5. On the left, the procedure for measuring the spatial uniformity of irradiance of the projector. On the right, the measured irradiance map.
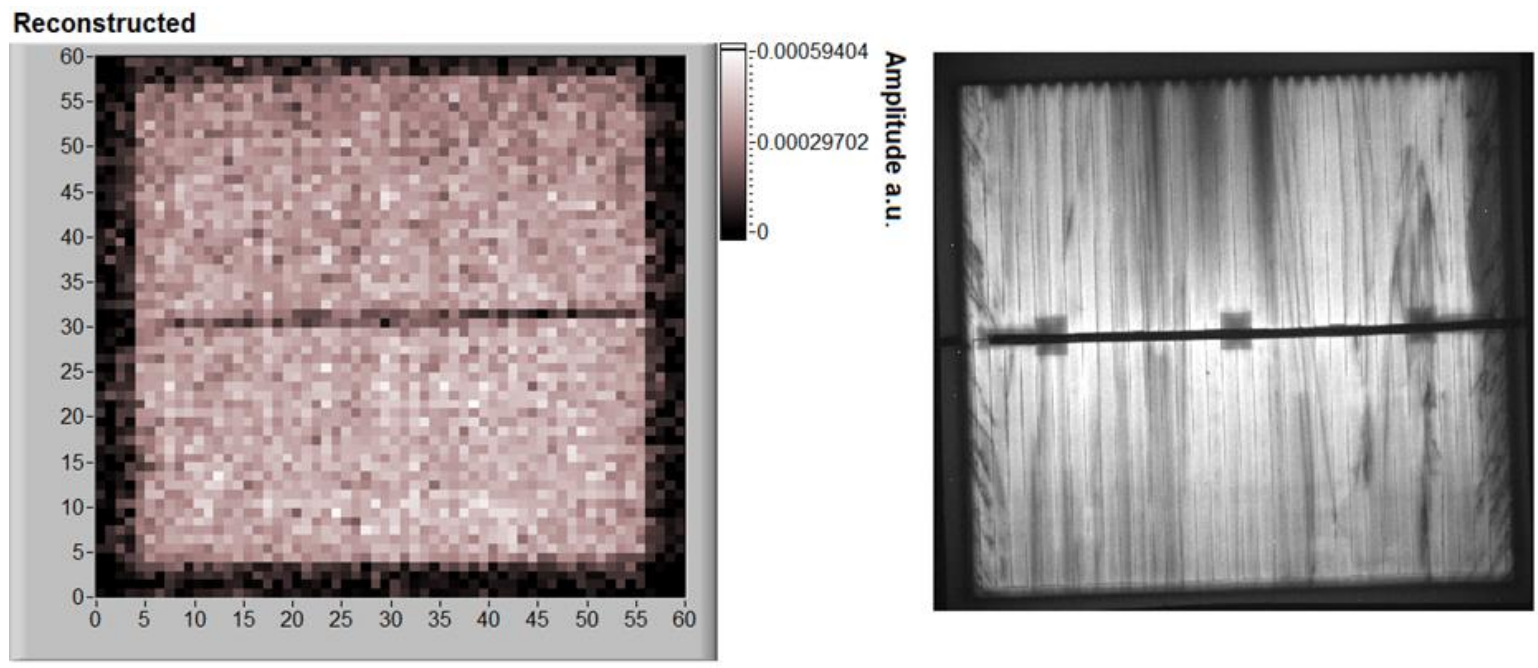

Figure 6.6. On the left, an initial $60 \times 60$ current map of a mc-Si cell, acquired with the unmodified DLP projector as a light source and by applying compressive sampling. On the right, an EL image of the same sample. 


\subsubsection{Projector unit modifications}

As discussed above, the projector utilises a colour wheel filter for splitting the white light source. This operation is well suited for usual projection utilities, but creates significant spectral and temporal variations of irradiance for PV characterisation applications. In order to overcome this limitation, the internal colour wheel of the projector was removed from the light path, by disassembling the projector. A picture of the projector main control board and some of its components is presented on the left of Figure 6.7, with the casing and protection lid of the projector detached. The position of the colour wheel within the projector before its removal is presented on the right of Figure 6.7. One initial obstacle found was that the projector would not operate with the colour wheel completely disconnected and removed, apparently for safety reasons, presenting an error signal.

To overcome this, the colour wheel is physically removed from the light path, but it is left connected electrically to the main board of the projector with all its sensors and cables. The colour wheel is placed outside the projector and it is still always spinning when the projector is turned on, but no longer affects the projected light. There are no safety related risks with this approach; the projector is still hermitically sealed by its protective cover and plastic lid so its inner parts are inaccessible; and there is no risk of injury due to the spinning colour wheel, since it is extremely light-weight and can be stopped with minimum force even by bare fingers. After this adjustment the projections are only black and white, but this is precisely as required in the intended application of CS here. A picture of the modified projector in operation is presented in Figure 6.8.
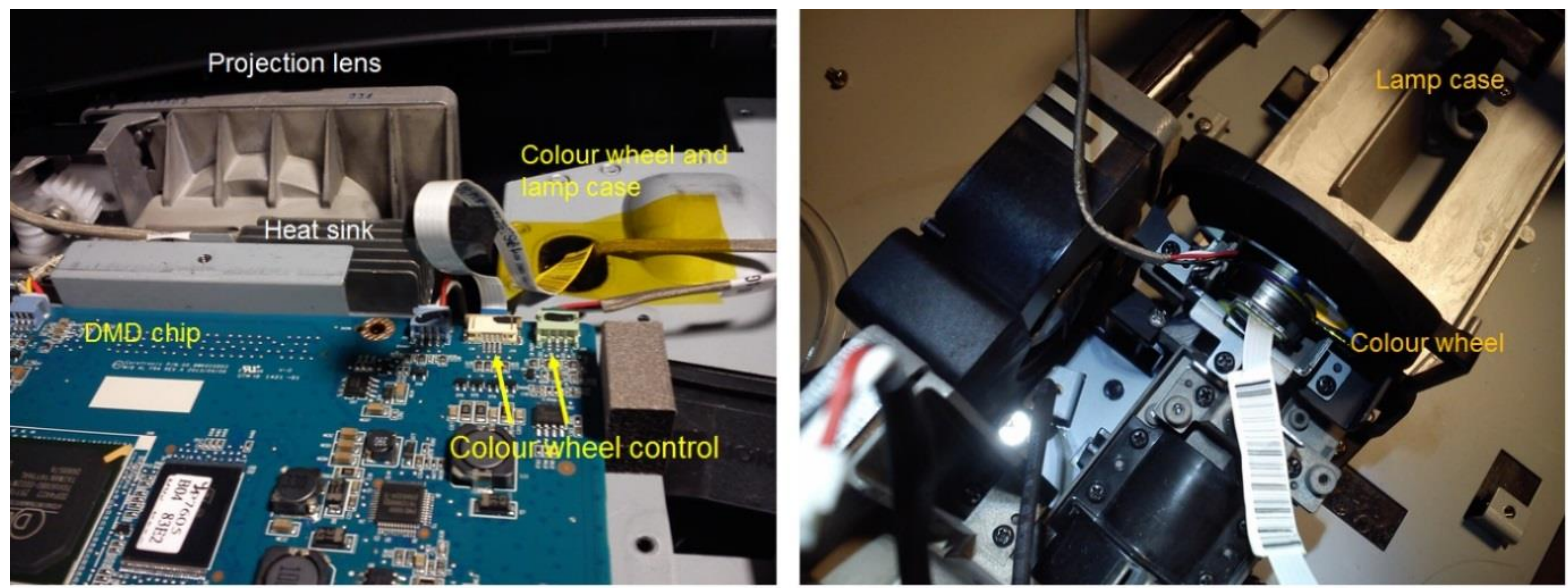

Figure 6.7. Pictures of the disassembly procedure of the projector. 


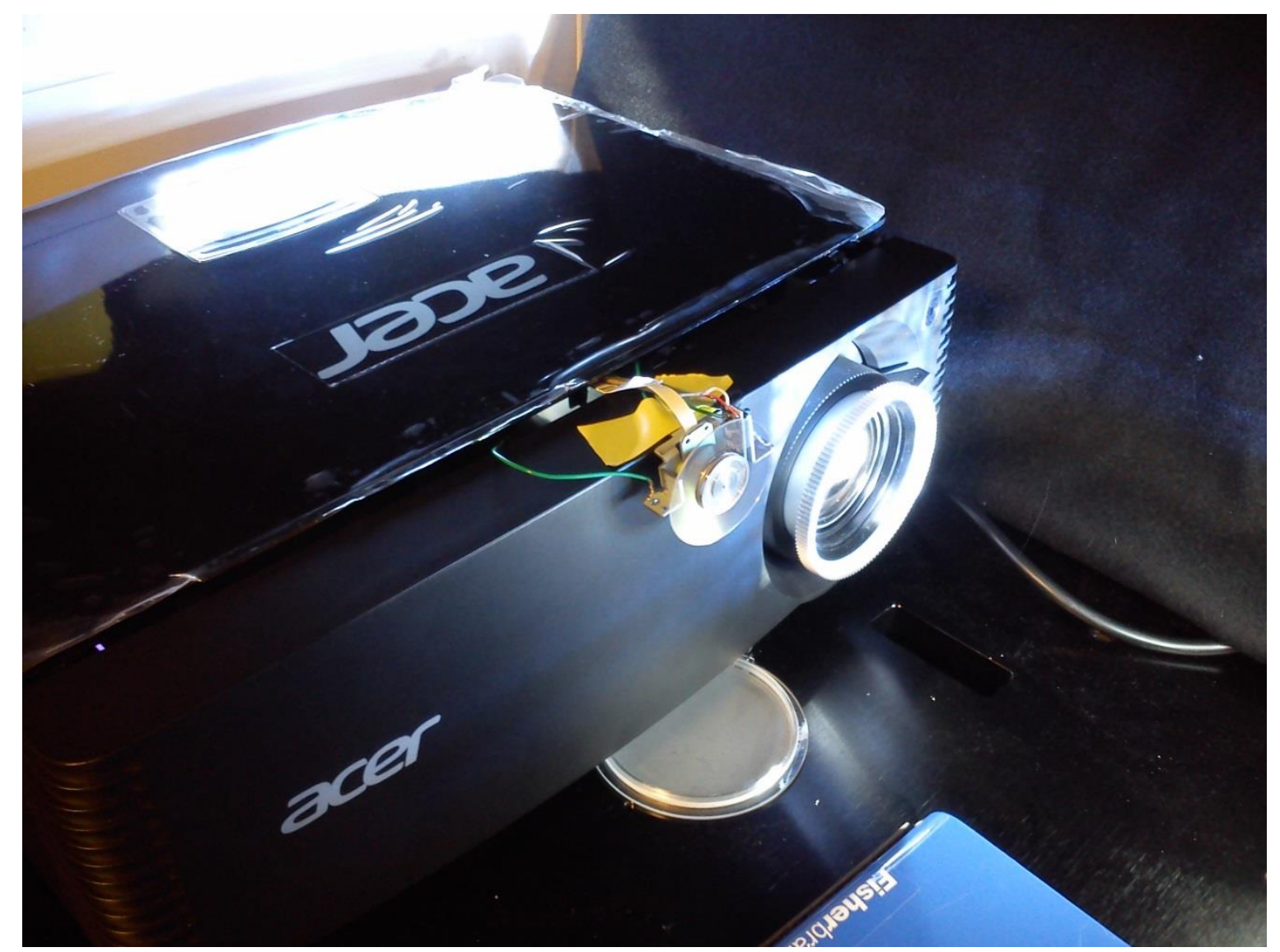

Figure 6.8. The DLP projector operating after the removal of the colour wheel from the light path.

\subsubsection{Custom PV module}

A custom 6-cell monocrystalline silicon (c-Si) module produced at CREST is used for testing the DLP projector current mapping system. This PV module is similar to the standard laminate production methodology used by most c-Si module manufacturers. Thus, apart from the number of cells and lack of surrounding aluminium frame, the module used is of equivalent construction and technology to commercial modules available on the free market. One additional feature is that the terminals of each individual cell were extended to the outside of the encapsulation, allowing direct contact with each cell. This enables a flexible contacting scheme, where tests are either run in a way to simulate commercial modules and all cells are contacted in series, or direct measurements are taken on individual cells. The PV module and its circuit diagram are presented in Figure 6.9. 

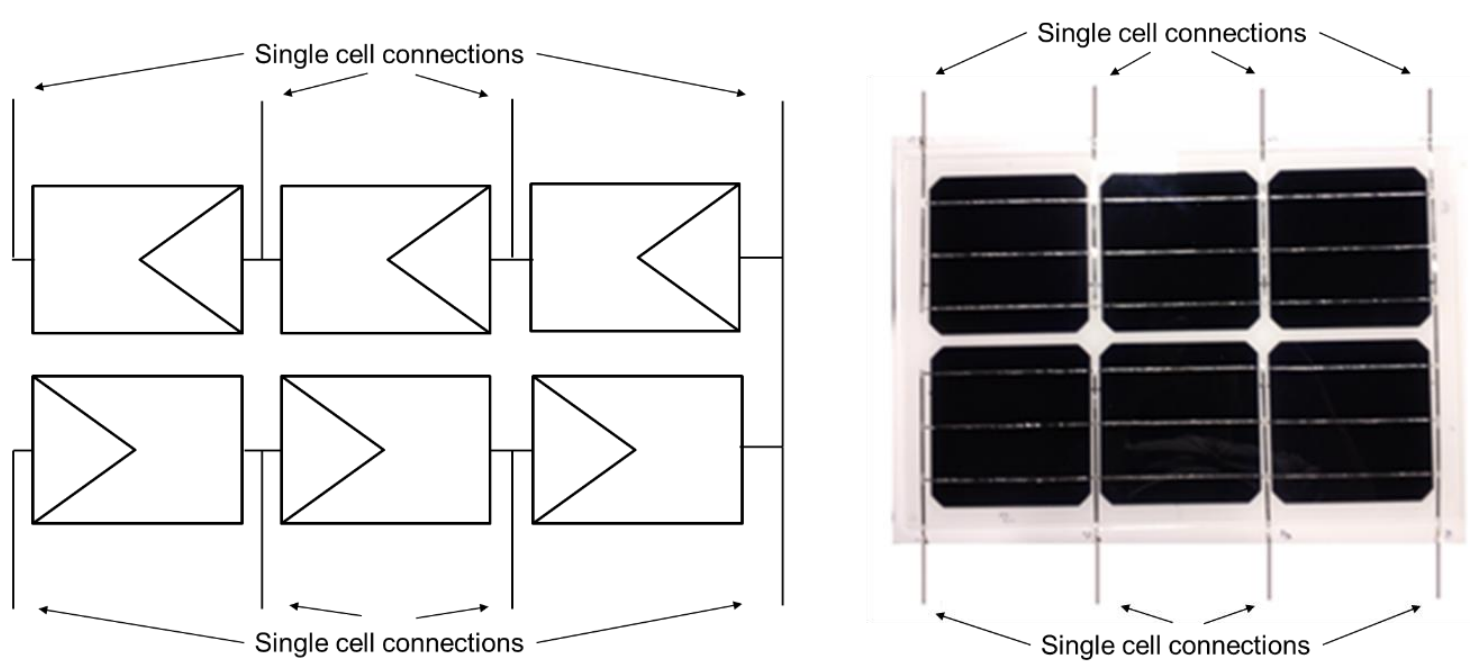

Figure 6.9. On the left, the circuit diagram of the c-Si PV module used in this work. On the right, a photograph of the custom mini-module. In both cases the external individual contacts for each cell are visible, for validation of experimental results.

The standard parameters of all cells of the mini module were measured in a commercial solar simulator (Pasan IIIb) at standard test conditions (STC) and are shown in table 6.1. It is apparent that all the cells exhibit almost identical performance. This is crucial for initial testing of the DLP current mapping system and in order to confirm the feasibility of current mapping with this system and the validity of measurements. Synthetic defects are introduced in a later stage, for a more realistic approach. No bypass diodes were included in the mini-module at this stage.

Table 6.1. STC parameters of the custom module used in this work and its individual cells

$$
I_{\mathrm{SC}}(\mathrm{A}) \quad V_{\mathrm{OC}}(\mathrm{V}) \quad I_{\mathrm{MPP}}(\mathrm{A}) \quad V_{\mathrm{MPP}}(\mathrm{V}) \quad P_{\mathrm{MAX}}(\mathrm{W}) \quad F F(\%) \quad n(\%)
$$

$\begin{array}{lccccccc}\text { Cell 1 } & 9.17 & 0.63 & 8.2 & 0.47 & 3.84 & 0.68 & 15.58 \\ \text { Cell 2 } & 9.09 & 0.63 & 8.35 & 0.46 & 3.83 & 0.67 & 15.54 \\ \text { Cell 3 } & 9.1 & 0.62 & 8.24 & 0.47 & 3.86 & 0.68 & 15.66 \\ \text { Cell 4 } & 9.1 & 0.62 & 8.42 & 0.46 & 3.87 & 0.69 & 15.7 \\ \text { Cell 5 } & 9.06 & 0.62 & 8.33 & 0.46 & 3.82 & 0.68 & 15.5 \\ \text { Cell 6 } & 9.16 & 0.62 & 8.21 & 0.47 & 3.84 & 0.68 & 15.59\end{array}$




\subsubsection{Signal variations and filtering}

After the removal of the colour wheel described above, the projector can produce only white light (or no light). In order to re-evaluate the signal, one of the cells of the mini PV modules was stably illuminated and the current measured with a sampling rate of 250000 samples per second. Initially, only white light is projected on the cell, with all the pixels set to the "on" state. Even without the colour wheel there are still periodic variations of intensity, as can be seen on the left of Figure 6.10. Projecting a black and white random pattern with $50 \%$ ratio of white and dark pixels results in the signal measured on the right of Figure 6.10. The shape of the periodic variation does not change at all and the intensity is much more stable, which is encouraging. The overall intensity (represented by the current measurement is proportional to shading levels (proportion of dark pixels over the cell area). The projector internal DMD control behaves as if the colour wheel is still in place and some intensity variations are inevitable. This resulting signal of Figure 6.10 is in reality the final result after some additional investigations and alterations made via the projector user interface settings, described below.

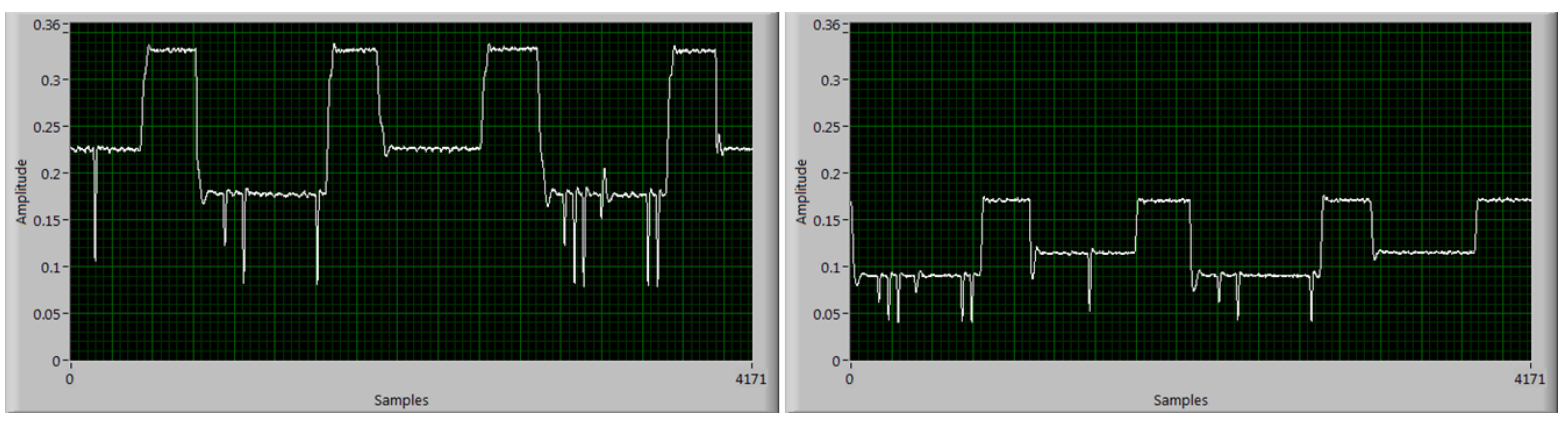

Figure 6.10. Variations of light intensity of the projector, for one period of the signal. On the left a white image is projected, on the right a random pattern with $50 \%$ shading.

The brightness, contrast and colour balance can be altered so that intensity is set as stably as possible. All values have a range from 0 to 100 . The contrast level is set to max here (100) so that a dark pixel is as dark as possible. In order to test the impact of brightness, a $50 \%$ shading random pattern was projected onto a PV cell. At maximum brightness (100), the dark pixels appear more grey than black and the signal of one period is presented on the left of Figure 6.11. By setting the brightness to minimum value, the signal becomes much more stable and the dark pixels are as dark as possible. For this reason minimum brightness was selected, the signal is presented on the right of Figure 6.11. 


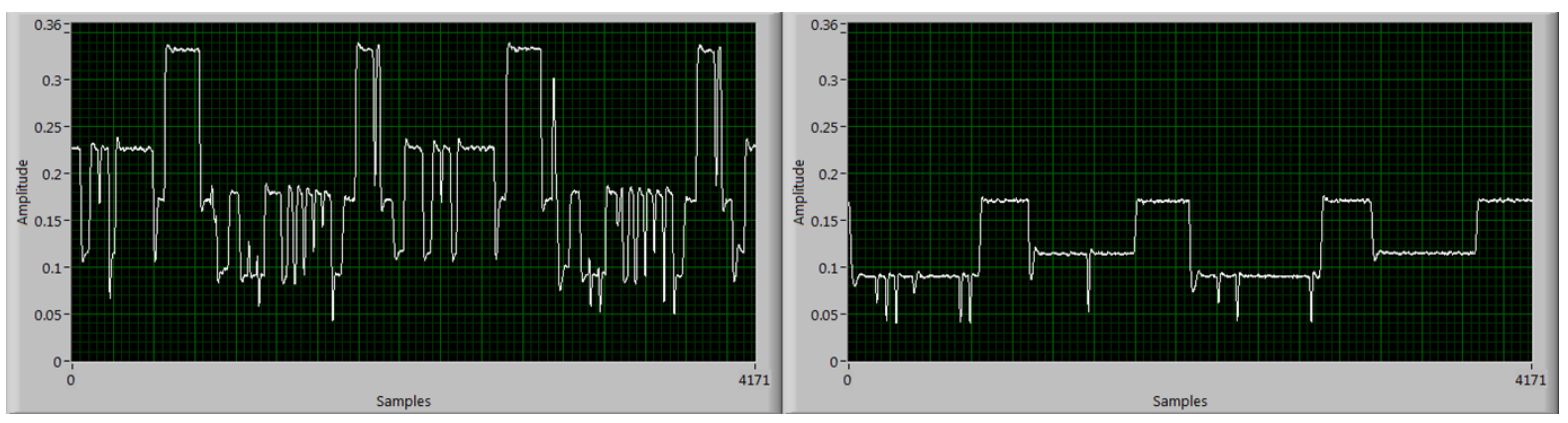

Figure 6.11. Variations of light intensity of the projector, for one period of the signal, while projecting a 50\% shading black and white random pattern. On the left, the brightness set at max value, on the right at minimum.

Another setting that was changed using the projector interface is the "colour temperature" setting. This setting controls the intensity of each of three colours, red, green and blue (RGB), within a range of 0 to 100 , apparently assuming that the colour wheel is in place to apply these settings. The signal in Figure 6.10 was acquired with this setting at RGB: 100-100-100. Even without the colour wheel, altering this setting has an impact on the signal. Different combinations of the "colour temperature" settings are presented in Figure 6.12. Any setting other than 100-100-100 makes the light intensity very unstable, with the signal being at zero levels for long times within one period. In practice these variations cannot be perceived by the human eye, the only visible effect is that the intensity seems significantly lower when removing a colour. For the remainder of the experiments the RGB parameter was set at 100-100-100.

$100-0-0$

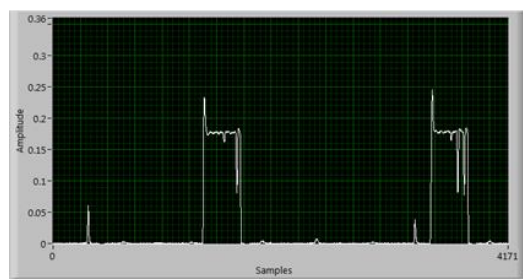

$100-100-0$

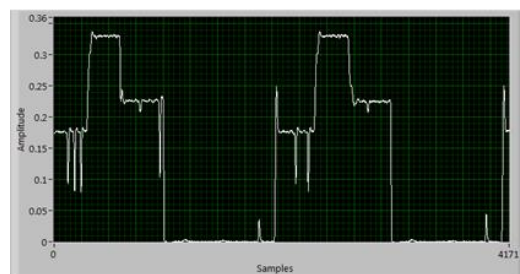

0-100-0

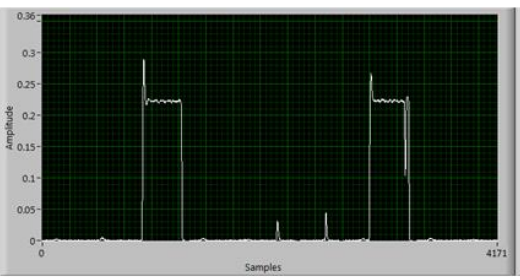

$100-0-100$

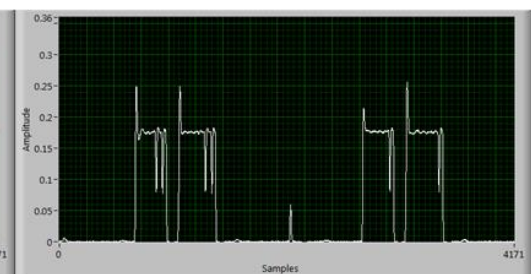

0-0-100

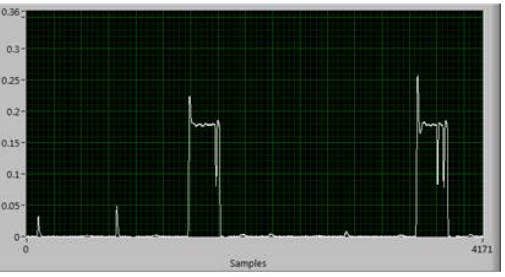

0-100-100

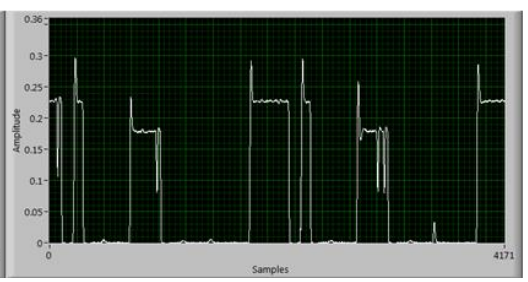

Figure 6.12. The signal within a period, with different combinations of RGB settings. 
The signal of each period is averaged and recorded, and each current measurement averages 10 such readings, for reducing measurement noise due to intensity variation. Using these settings, the SNR achieved is approximately 1000 for compressive sampling, which seems not to be enough according to the findings of the previous chapter. In addition, the sampling rate is extremely slow, around 2 samples per second, due to the signal instability and the long integration times required to accommodate elements of the projector DMD control that are not accessible to modify. In order to improve the signal, additional data filtering is applied. After the sampling procedure, only readings with values above $90 \%$ of the maximum signal value are kept and the rest are rejected. Around $70 \%$ of the readings are thus rejected, however the signal is significantly more stable. This brings the SNR of the measurements to slightly over 2000, still lower than ideal for CS measurements. Nevertheless, low resolution CS current mapping of PV modules can still be achieved, in order to assess the feasibility of the concept.
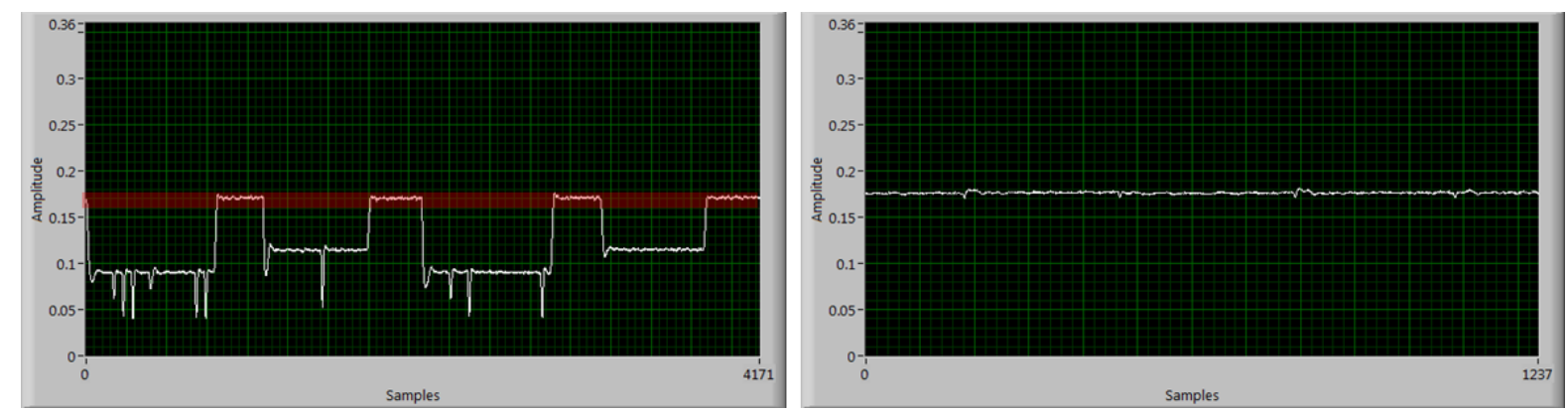

Figure 6.13. The filtering procedure applied to the signal; only readings above $90 \%$ of the maximum value are kept, while the rest are rejected.

\subsubsection{Experimental setup and procedure for CS current mapping}

After considering the results of the above investigations, the experimental setup for CS current mapping with the DLP projector is based on the configuration described in 5.3.1. The sample is placed vertically at a fixed position on a custom mounting base and a Keithley 2420 sourcemeter loads the PV sample at a constant set voltage bias. The DLP projector projects the necessary patterns for compressive sampling onto the device under test, while its colour wheel has been removed. A NI DAQ card reads the current response of the sample for each pattern, with the utilisation of a shunt resistor, as presented in Figure 6.2. The reconstruction of the current map is achieved in MatLab, using the $\ell 1$ minimisation algorithm, as described 
in the previous chapters. The $\ell 1$ minimisation algorithm is used in all cases in this chapter, as with these levels of noise there is no significant difference when using the OMP algorithm, which needs much longer reconstruction times. The projector parameter settings are minimum brightness, maximum contrast and maximum colour level for all three colours (RGB), as described above. A diagram of the experimental layout is presented in Figure 6.14. All the experimental procedure is controlled with a LabVIEW software written specifically for this application. A sensing matrix is generated beforehand and is loaded into the program before measurements start.

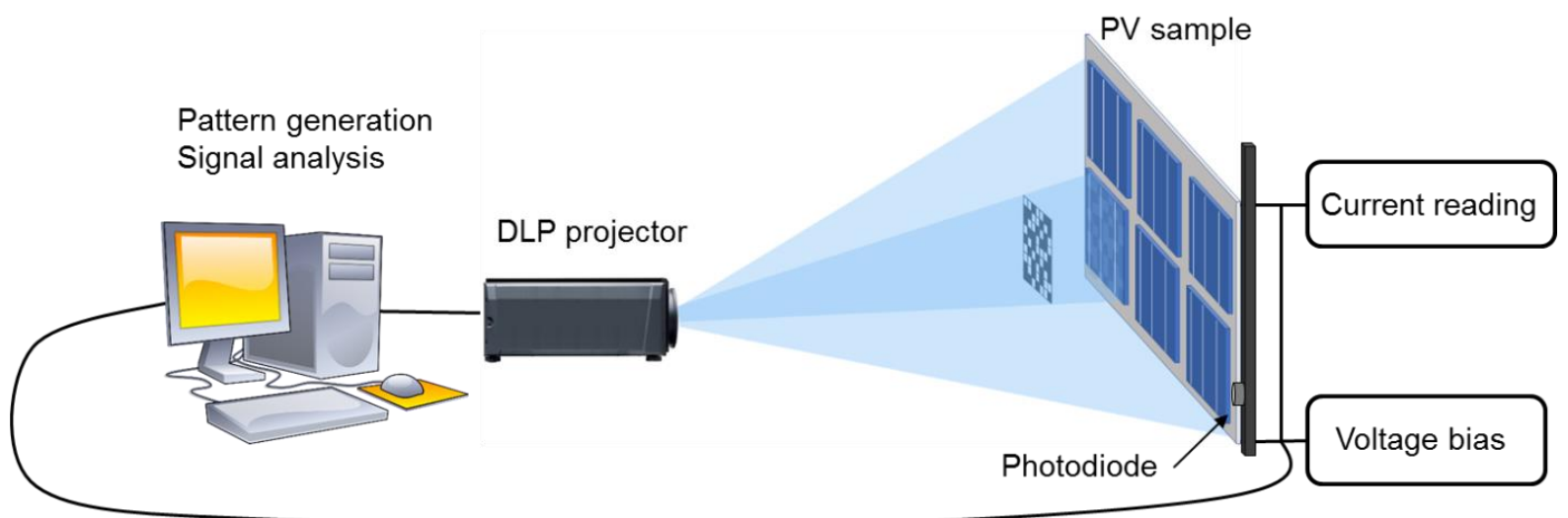

Figure 6.14 The experimental layout. An image is projected onto the PV device. The part of it covering a single cell includes the specific pattern for compressive sampling while the rest of the cells are fully illuminated.

The average absolute value of the irradiance on the sample plane is approximately 30 $\mathrm{W} / \mathrm{m}^{2}$. This is significantly lower than that used in standard test conditions for PV modules $\left(1000 \mathrm{~W} / \mathrm{m}^{2}\right)$, but it allows a reasonable basis for the acquisition of valid measurement results in this study, to evaluate the potential of this system. The long term stability of the light source is also monitored simultaneously with the measurements, this is achieved using a photodiode installed next to the PV sample under test.

With the use of the custom mini-module, the CS current mapping system based on the DLP projector can be tested for both single PV cells and PV modules. In the case of PV module measurements, the binary patterns are projected on the cell under measurement while the other cells in the module are fully light biased. This is necessary to ensure the cell under measurement is the limiting cell of the module [78]. As a result, the current measured at the contacts of the module is the current of the cell under measurement. 


\subsection{CS current mapping measurements}

\subsubsection{Current mapping results for individual, large area cells}

As a first step, current maps of a single PV cell of the mini module were acquired to verify the functionality of the system. A single cell of the mini module was directly connected using the custom connections built into the module. The sequence of random patterns for compressive sampling was projected on this cell as shown in Figure 6.15. An EL image of this cell is also presented in the same figure. The cell was kept at OV bias during CS measurements so that it is measured under Isc conditions. The sensing matrices used had $40 \%$ of the pattern pixels bright and each pattern had a size of $100 \times 100$ pixels. Similar to the previous chapter, this size of sensing matrices was selected for simplicity of implementation and for achieving a relatively fast reconstruction process. The choice of sparsity levels is a compromise between SNR and reconstruction performance.
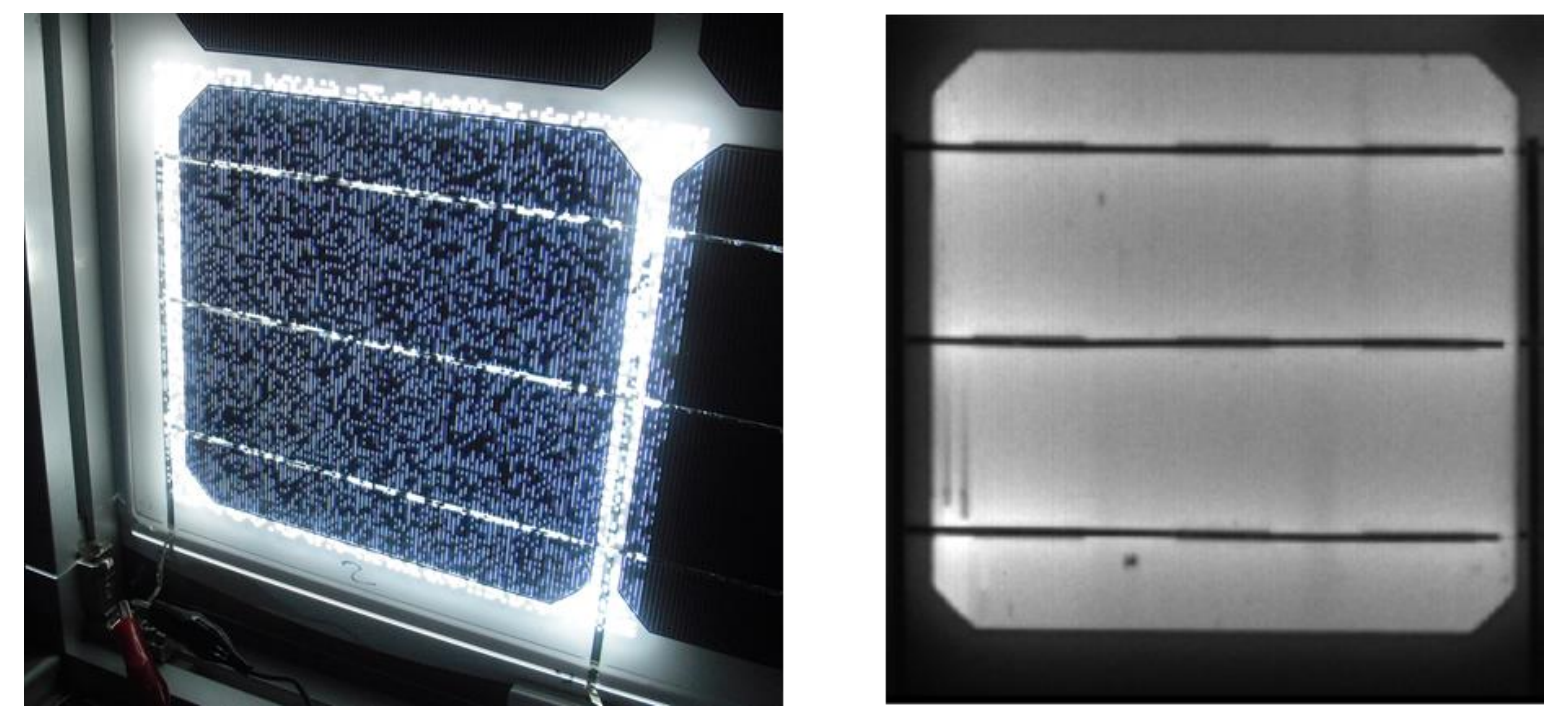

Figure 6.15. The random pattern sequence is projected on one of the cells of the mini module which is directly contacted. An EL image of the cell is also presented on the right.

The current maps acquired using this setup are presented in Figure 6.16. The percentages are the ratio of measurements taken to the total number of pixels of the current map. The feasibility of the method is confirmed as current maps can be produced with this approach. Although the resolution is not high enough to distinguish the fingers of the cell, the busbars are clearly visible. Due to the low resolution and the broad spectrum of the light of the projector, the defects observed in the EL image of Figure 6.15 are not detectable. The white light does not penetrate deep enough into the bulk of the device to reveal defects visible in EL imaging. In addition, measurements are realised at $I_{\mathrm{sc}}$ conditions which means 
series resistance effects will not be visible (whereas they are inherent in the EL images). Thus, the acquisition of a very uniform current map was expected. The resolution achieved in these results is approximately $1.5 \mathrm{~mm}$ per pixel and as a result the fingers of the cell cannot be observed. As for the small area experimental setup, in order to reconstruct the current maps, fewer measurements than the number of pixels of the current map are needed. As can be observed, when acquiring more than 4000 measurements (40\%) no significant further improvement is achieved for the reconstructed current map.
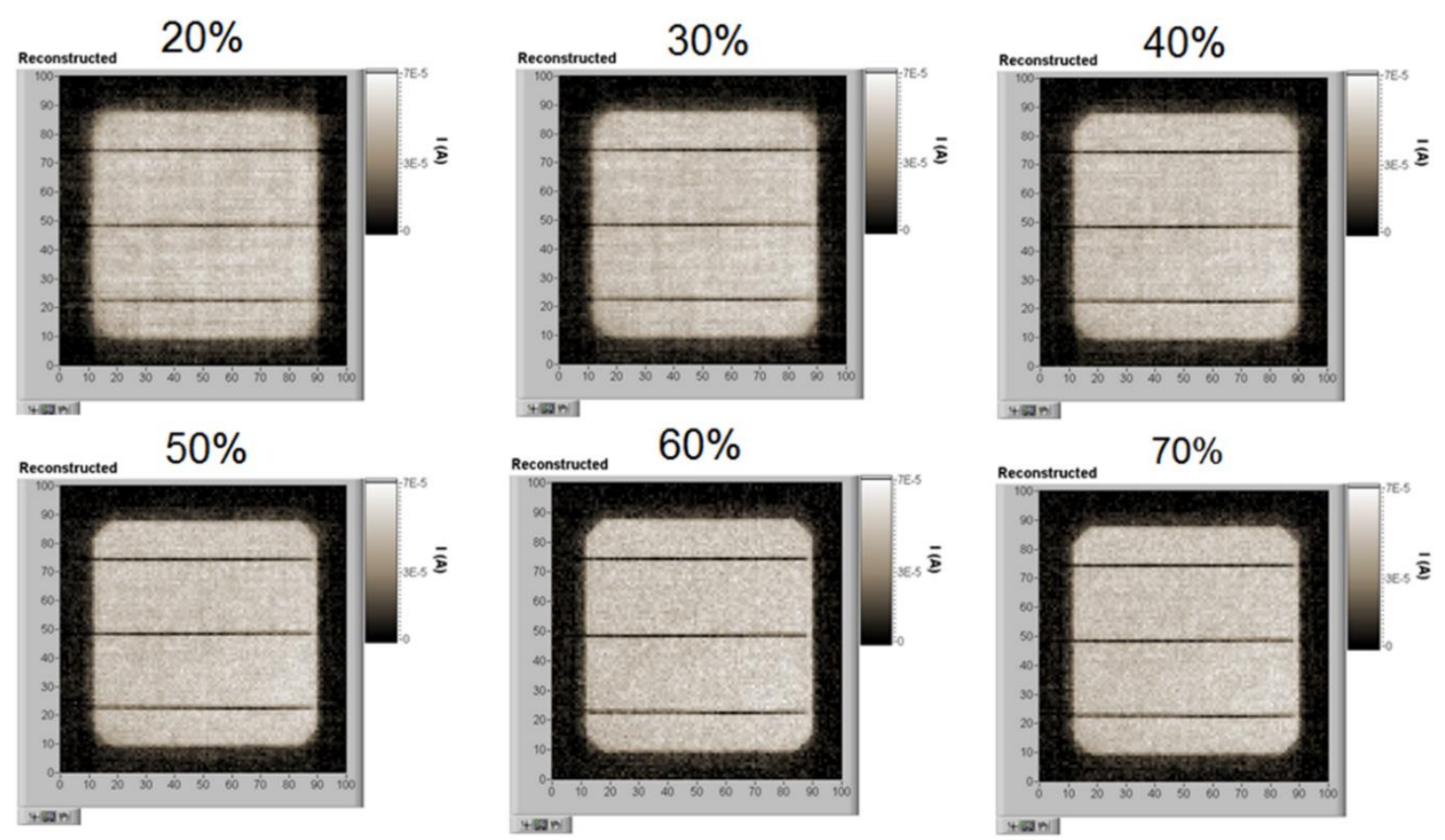

Figure 6.16. CS current maps of a single c-Si cell, individually contacted. Number of measurements is expressed as a ratio of number of measurements acquired to the number of pixels of each image (10000).

The reconstructed current maps are noisy, still affected by the temporal variations of intensity discussed previously. Unfortunately, the measurement SNR achieved in this system when using compressive sampling is approximately 2000 only and this is reflected in the results. However, due to the low levels of irradiance on the sample and the relatively high levels of noise, it was not possible to realise even a poor quality point by point current scan for a direct comparison. This was expected from the findings of the previous chapter; with such levels of noise a meaningful point by point scan on such a full size cell is impossible. However, this shows in practice the usefulness of compressive sampling when a system contains high levels of noise, as in this case. In addition, in this first case the current maps 
were acquired with a very simple setup and a very unstable light source, which suggests good potential for future improvement of the method.

For an investigation of non-uniform performance devices, defect features were synthetically added to individual cells by adjusting black non-transparent covers (masks) on the cells' surfaces. Different cases of shapes of covered areas were tested; a triangular area at the bottom of a cell, which could represent a disconnected area of the cell; a rectangular area on the upper side of a cell, which could simulate broken fingers; and a thin strip and a bent line on a third cell, which could represent cracks. The results are presented in Figure 6.17.
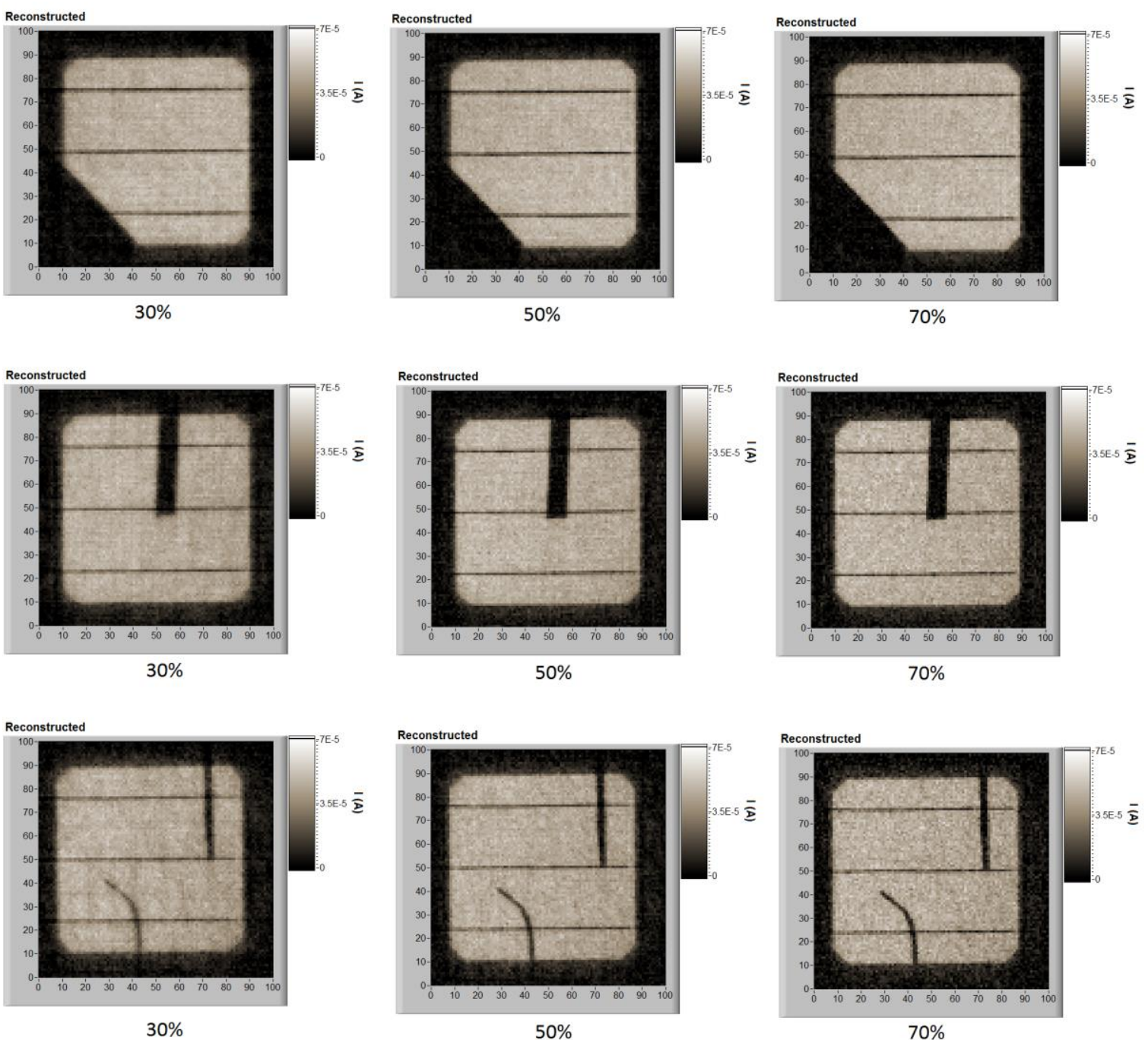

Figure 6.17. CS current maps of individual c-Si cells. Number of measurements is expressed as a ratio of number of measurements acquired to the number of pixels of each image (10000). 
These large features are easily detected by the CS current mapping method, even when acquiring $30 \%$ of the measurements a point by point scan would need. By acquiring more measurements the current map becomes sharper, although there is still measurement noise and increased pixelation. These results demonstrate that the CS current mapping method introduced in the previous chapter for small samples is scalable in order to measure larger cell samples. With the primitive system built based on the commercial projector it is difficult to achieve higher resolution. In addition, the broad spectrum of the white light of the lamp in the visible range does not reveal any detail from the bulk region of the cell, beneath the surface. However, this investigation suggests that a simple custom projection system with a single wavelength light source could offer an alternative solution for current mapping of large wafer based solar cells. In addition, as it is presented in the next section, such a system can achieve current mapping of PV modules comprising interconnected cells, which is not straightforward with current established LBIC systems.

\subsubsection{Voltage bias considerations for current mapping of modules}

For applying CS current mapping to a PV module, some implications of the applied voltage bias have to be considered. When all the cells of the module are almost identical as in this case presented, by setting the PV module to short circuit conditions ( $0 \mathrm{~V})$ and illuminating the full module, each cell should individually be at short circuit conditions also. With the projector as the light source, some small differences in the voltage operating point of each cell should arise due to illumination non-uniformities. When CS sampling is applied to one cell of the module while the rest are fully illuminated and the PV module is set globally to $0 \mathrm{~V}$, it is expected that the cell under measurement is actually reversed biased since it is shaded, while each of the others will be slightly forward biased, resulting in a sum of $0 \mathrm{~V}$ for the module.

To verify this behaviour, all the cells of the custom mini-module were individually contacted and their voltage was measured while the PV module was biased at $0 \mathrm{~V}$. A sequence of 100 shading patterns was projected on one of the cells, each pattern decreasing shading level (proportion of dark pixels randomly distributed over the area of the cell) by $1 \%$. This means that the first projection fully shades one cell of the PV module while the other cells are fully illuminated, while the $100^{\text {th }}$ projection fully illuminates all of the cells. This procedure will demonstrate the effect of application of the compressive sampling sensing matrices on 
the voltage bias of individual cells of the module. This test is applied to cell 1 (indicated in Figure 6.18) and the results for the voltage operating points of all cells are presented on the right of Figure 6.18. On the left of the same figure, the voltage bias levels for all cells are given when all the cells are fully illuminated. This shows the effect of the non-uniformity of the projection, which is already described earlier. Cell 4 receives the highest amount of irradiance and it is slightly more forward biased than the rest, while cells 1 and 5 are slightly reversed biased, as the irradiance intensity at the edges of the projection is slightly lower. Nevertheless, as it will be demonstrated this issue does not prevent measurements to be applied.
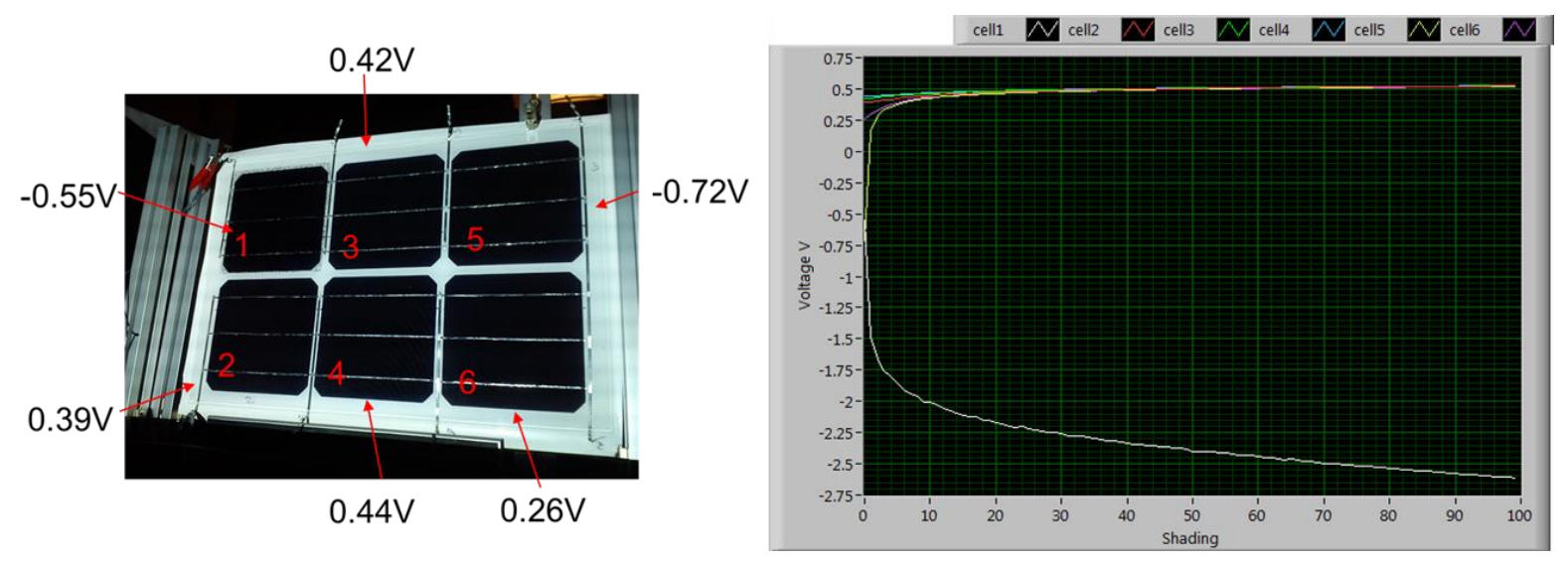

Figure 6.18. On the left, the voltage bias of each cell when all the cells are fully illuminated and the module voltage is set to $0 \mathrm{~V}$. On the right, the voltage levels of each cell when cell1 is shaded with different shading levels.

Regarding the voltage bias levels for each cell presented on the right of Figure 6.18 , it is clear that while shading is increased, the shaded cell becomes reverse biased while the rest of the cells are forward biased as expected. This also shows that initial small non-uniformities of irradiance have no impact on voltage bias levels if one of the cells is shaded. In the case where one cell is underperforming compared to the others in the same module, it is reversed biased even when unshaded. To confirm this, similar defect-emulating masks to the previous section were used to reduce the output of some of the cells of the module. Three of the cells were covered with different sizes of these masks, as presented in Figure 6.19. The largest mask was placed on cell 3 . When all the cells are illuminated, the cell with the largest mask is strongly reversed biased, while all the others are forward biased at very similar levels as can be seen in Figure 6.19 (right). Running the same procedure as before and measuring the voltage of each cell while shading patterns are projected onto cell 1 , the graph on the right of Figure 6.19 is acquired. 

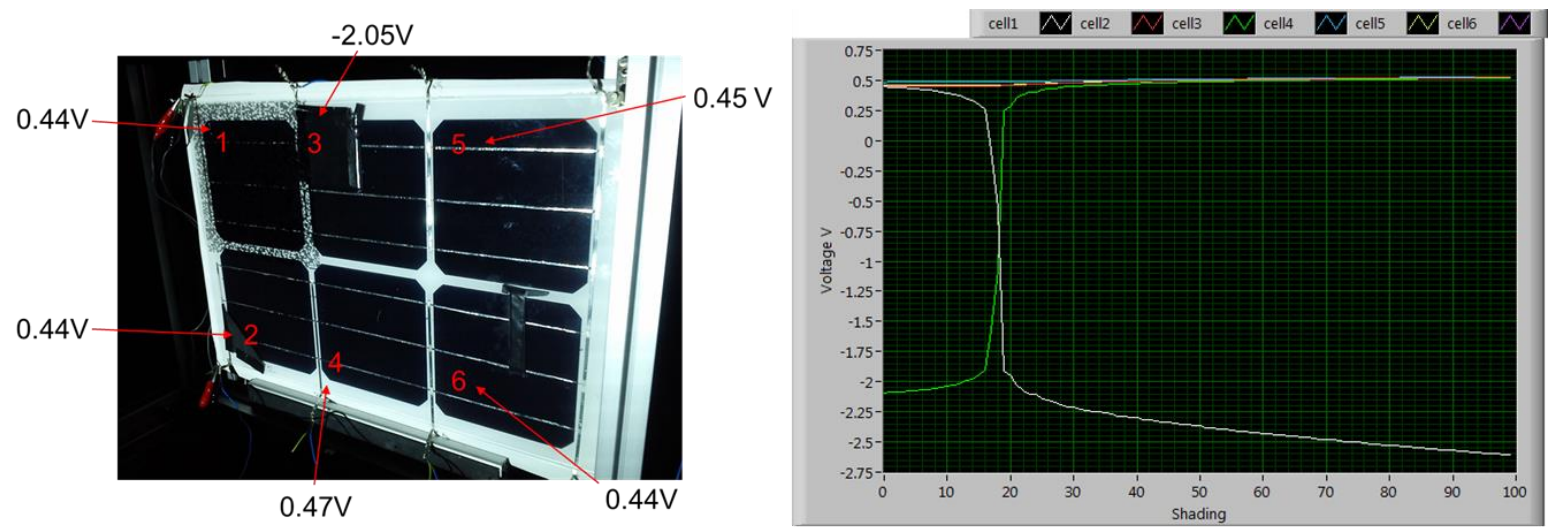

Figure 6.19. On the left, the voltage bias of each cell when all the cells are fully illuminated and the module voltage is set to $0 \mathrm{~V}$. Masks are applied on some of the cells. On the right, the voltage levels of each cell when cell1 is shaded to different levels.

These results show that even if several cells of a module are underperforming, only the worst of them will be reversed biased. By increasing the shading levels on any of the rest of the cells, there is a point above which the shaded cell (and not the underperforming one) becomes reversed biased. This means that when applying CS current mapping, a forward voltage has to be applied to the module to bring the cell under test to operate close to short circuit conditions. To test this approach, the same measurements as above were applied but this time the voltage bias was set to $2.5 \mathrm{~V}$ across the module terminals. This was not selected arbitrarily, but is just below the value of 5/6ths of the $V_{O C}$ of the module ( $V_{O C}=3.15 \mathrm{~V}$ ) when all the cells are illuminated. Since the in the patterns used for compressive sampling $40 \%$ of the pixels are at the "on" state (60\% shading), the cell that is measured will be close to $0 \mathrm{~V}$ when $2.5 \mathrm{~V}$ forward bias is applied to the module. This is also demonstrated in Figure 6.20. In this case of measuring cell1, by applying $2.5 \mathrm{~V}$ forward bias to the module and for $60 \%$ shading, cell1 is close to short-circuit conditions (slightly forward biased $\simeq 0.07 \mathrm{~V}$ ). When applying CS measurements to the other cells, the forward voltage bias of the cell under test is in the region of $0.02 \mathrm{~V}-0.08 \mathrm{~V}$. Since in a PV module where individual cell contacts are inaccessible these direct measurements will not be possible, a module forward bias of $(N-1) * V_{O C}$ should be applied as a rule of thumb, when using sensing matrices of around $40 \%$ sparsity as in our case (where $N$ is the number of cells in series within the module). This ensures that the cell under measurement is always close to short-circuit conditions, or at least only very slightly forward biased. 


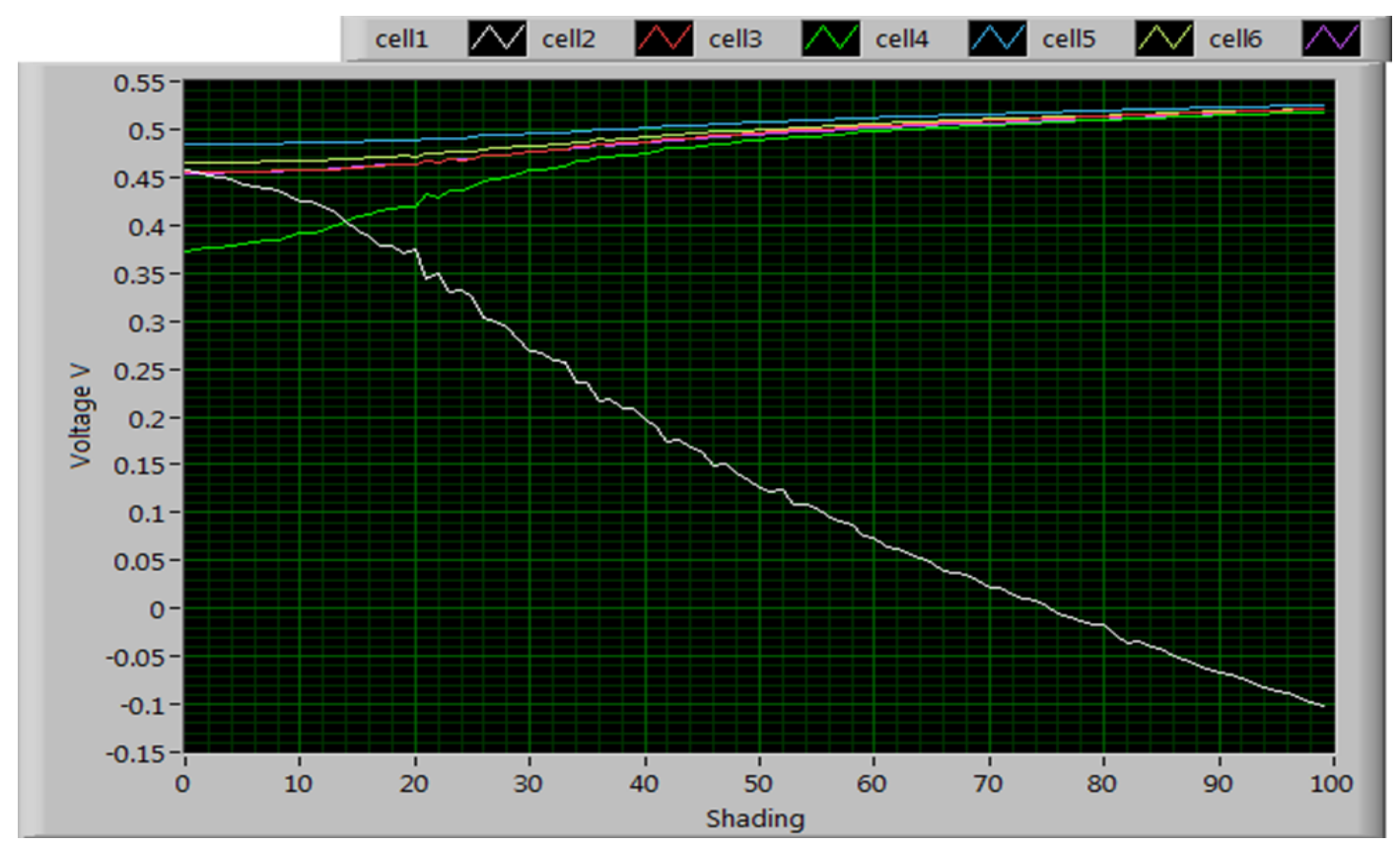

Figure 6.20. The voltage level of each cell of the custom module, when cell1 is shaded with different shading levels and the module is kept at $2.5 \mathrm{~V}$ forward bias.

In addition, provided that any cell of the module does not underperform by more than $60 \%$ of the best cell, CS current mapping measurements will be valid as the limiting cell will be that under measurement. When one of the cells has an even lower performance, sparser sensing matrices should be used for meaningful measurements. This may decrease the SNR ratio of measurements but this approach is necessary for applying this method successfully.

\subsubsection{Current mapping results for PV modules of interconnected cells}

Following the individual cell test cases and the voltage bias considerations, the next step is to test whether CS current mapping of PV modules is feasible practically. The same simple system based on the DLP projector is used. The mini module used is the same as presented in the previous section. Each cell was sampled with the necessary sensing patterns consecutively, while the others were fully illuminated. All the cells were connected in series and the current is measured by contacting only at the PV module terminals. The procedure is illustrated in Figure 6.21, where the patterns are projected onto one of the cells, which is the cell that is measured. As a result, the measured current at the contacts of the module is the current of the limiting cell under measurement. As 100 by 100 pixel random patterns were used, the reconstructed current map of the entire PV module consists of 60000 pixels. Due to the large 
integration and sampling time for each pattern necessary because of the non-customisable aspects of the light source described previously, measurement time was approximately 30 minutes for each cell. The reconstruction process is performed offline, using the $\ell 1$ reconstruction algorithm. This means that as soon as measurement acquisition of one cell has finished, the reconstruction process starts and the patterns move to the next cell at the same instant. For these measurements, the module voltage bias was set at $2.5 \mathrm{~V}$, using the approach described in the previous section. If no voltage bias is applied, acquiring a current map is not possible and reconstructing the measurements results in just images of snow.

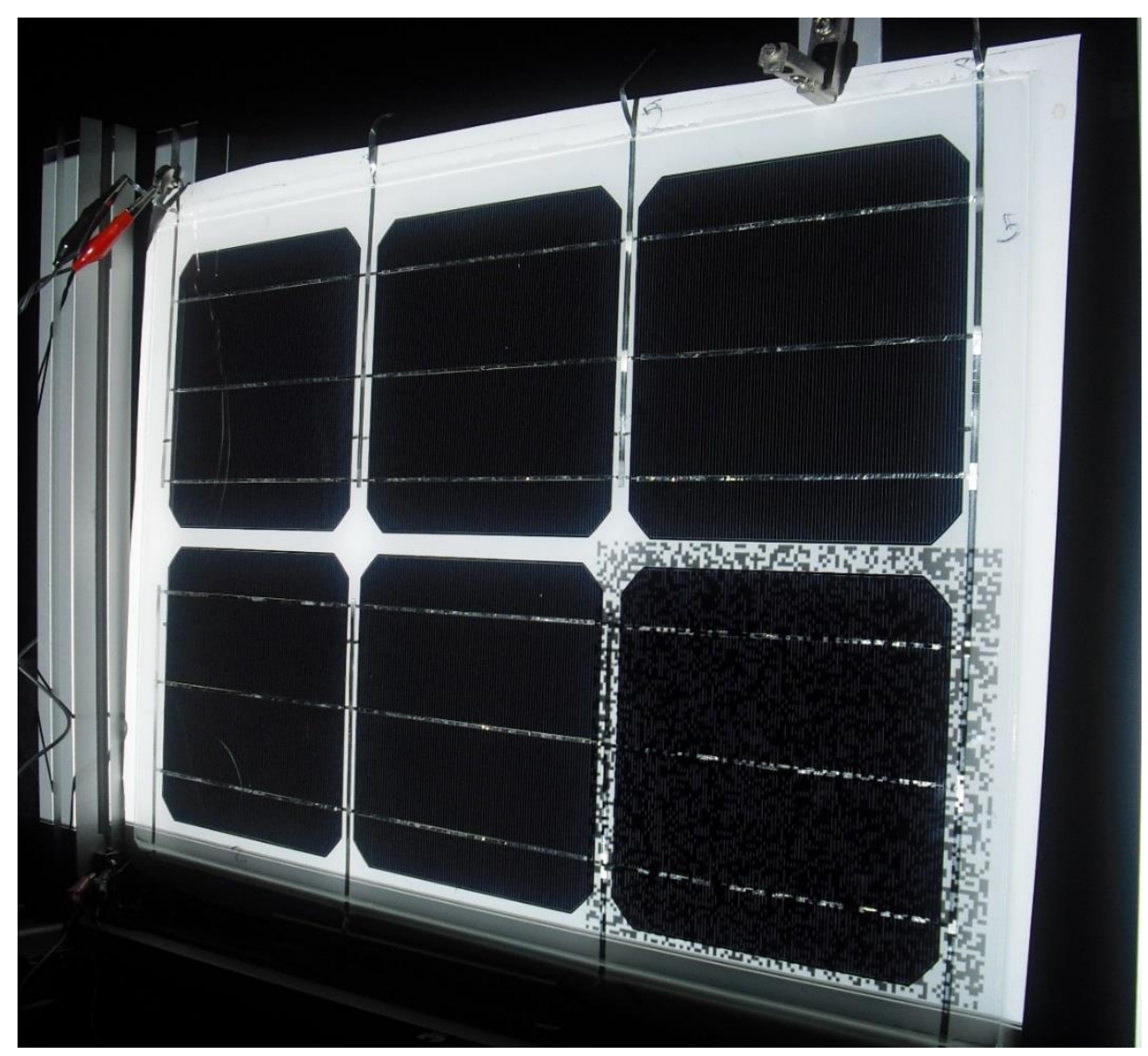

Figure 6.21. The 6-cell mini module used for these experiments. Patterns are projected on each of the cells in turn (bottom right cell shown here) while the others are fully light biased

The reconstructed CS current map of the 6-cell PV module is presented on the right of Figure 6.22. On the left of the same figure an EL image of the PV module is included for reference. The current map was acquired with 30000 measurements, an undersampling level of $50 \%$ of what a point by point scan would need. The noise levels are significantly increased compared to the single cell case, but this is expected when contacting the PV module and not 
individual cells, where current from the rest of the cells affects measurements [78]. The SNR value in this case is reduced to approximately 1000. An additional reason for this noise increase is also the larger active area. Moreover, the slightly non-uniform irradiance can be observed; the small current signal decrease of the top right cell is clear when compared to the bottom left cell. In a custom designed projection system for CS current mapping, a uniformity calibration could be applied in order to correct for such non-uniformities in a future improvement.

\section{EL image}

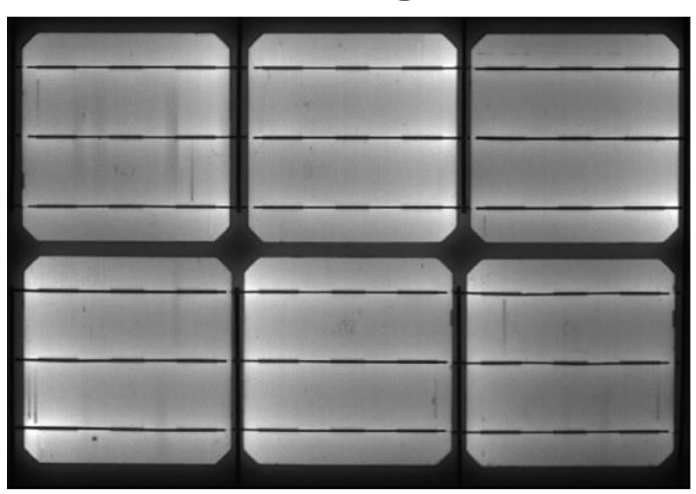

CS Current mapping

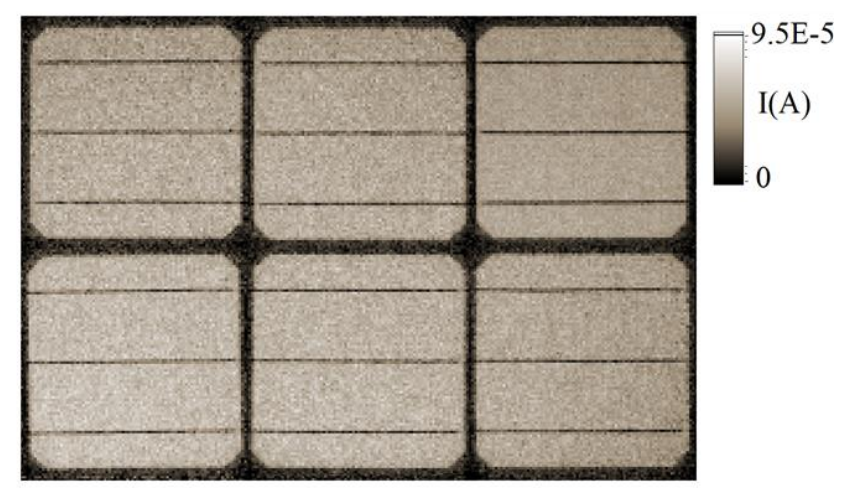

Figure 6.22. EL image and CS current map of the 6 cell PV module used in these experiments. The CS current map is produced having acquired 30000 (50\%) measurements

These initial results using the custom mini module show that the projection approach for current mapping is a very convenient way for acquiring current maps of modules. No mechanical shading is required to bring the cell under test to limiting conditions as in [78], where manual shading was used. The sampling patterns themselves not only realise the measurement but also provide the necessary shading that ensures the current limiting conditions.

These features become more useful when the performance of each cell in the module is slightly different. This causes current mismatch, which would make current mapping with a conventional LBIC system impossible, even when applying partial shading on the cell under measurement. To emulate this case of slight cell mismatch, masks were applied over some of the cells as in the previous case when individual cells were measured; a triangular area at the bottom of cell 2, a thin strip and a bent line on cell 6 and a much larger rectangular area on the upper side of cell 3. The masks are visible in the photograph on the left of Figure 6.23. After these changes, the module has one cell seriously underperforming, while the other masked cells will just slightly underperform. As a result, there are serious current mismatches 
in the module. A voltage bias of $2.5 \mathrm{~V}$ is once again applied to the module so that the measured cell is operating close to short-circuit conditions. The same procedure as in the previous case was followed and the resulting current map for the module is presented on the right of Figure 6.23, with an undersampling level of 50\% (30000 measurements, compared to 60000 pixels of the current map).
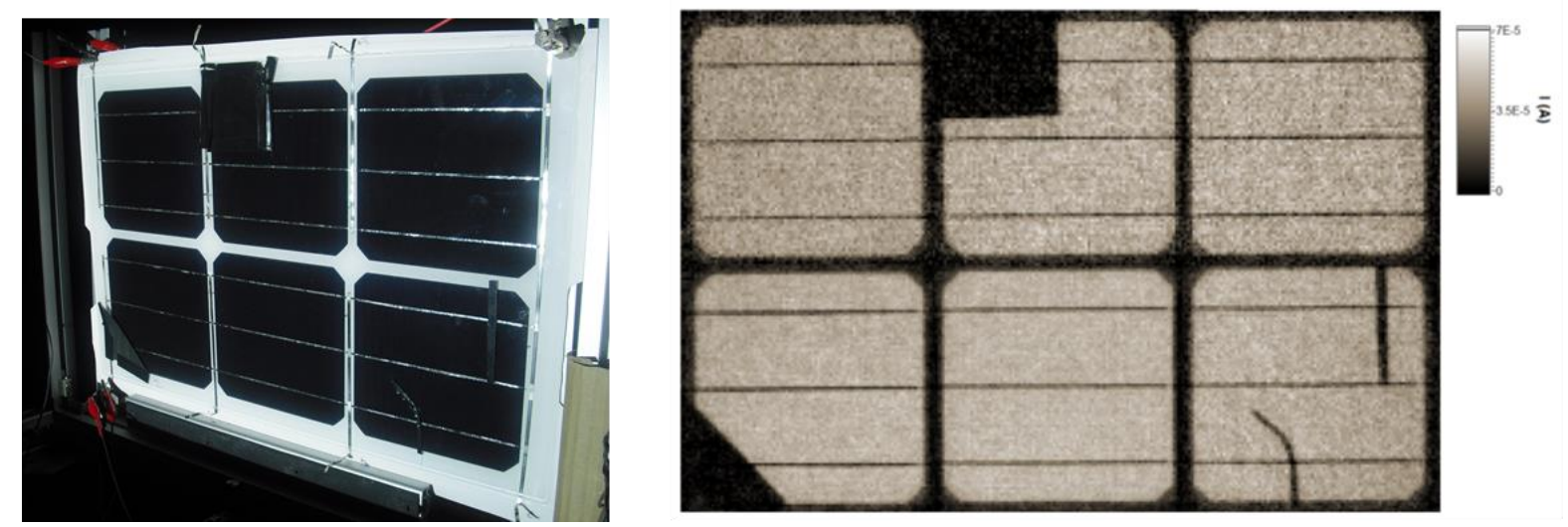

Figure 6.23. On the left, a photograph of the PV module with the attached masks to simulate underperforming cells. On the right, a CS current map of the module, with 50\% undersampling.

The results show that the method works, even in the case when there are current mismatches between the cells of the unshaded module. In reality, mismatches may exist in commercial PV modules, although such high levels of underperformance are more likely to appear in aged or damaged PV modules and not brand new ones. Some increased noise is visible in this case, which increases pixelation issues in the reconstruction process. This is more likely due to the current mismatch influencing measurements. Such issues were also observed in the case of LBIC measurements for PV modules with additional partial shading, where defects of individual cells were masked when contacting the module [78]. In the case of CS current mapping where patterns are projected on the cell under measurement, measuring the global current, this just increases the noise levels of the reconstruction process. 


\subsubsection{Bypass diodes and I-V curve considerations}

In this experiment the same custom PV module is used as in the preceding sections, with 6 PV cells connected in series. However, commercial PV modules always have by-pass diodes installed in order to avoid damage from overheating of underperforming cells and minimise power losses in such cases. By-pass diodes separate the cells of the same module into different sub-strings. This has implications when applying CS current mapping to a PV module with by-pass diodes, since they provide alternate current paths for locally reverse biased cells or cell sub-strings. To establish what modifications to the CS method would be necessary in such a case, two by-pass diodes were added to the custom PV module used here. The PV module now consists of two sub-strings of three cells each, with a bypass across each sub-string. This was straightforward to implement since the contacts of each cell were already extended outside the module. The masks on three of the cells used in the previous section were also applied. The configuration of the PV module with the bypass diodes and defectemulating masks is presented in Figure 6.24.

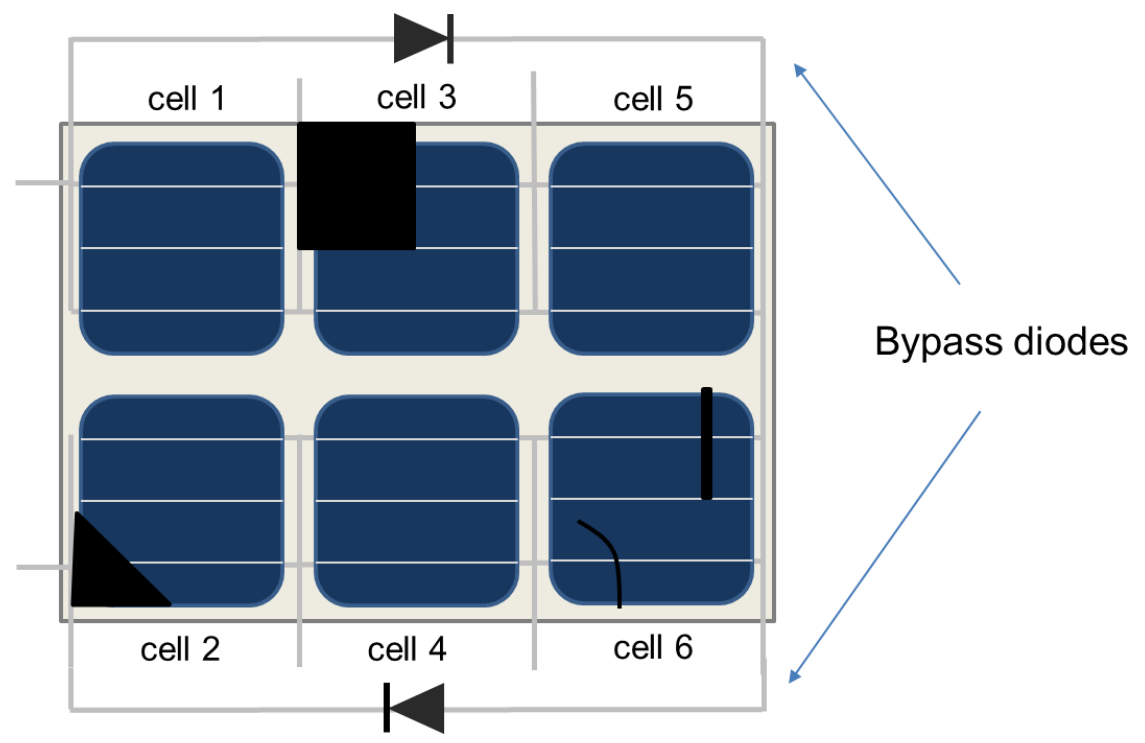

Figure 6.24. The configuration of the custom PV module of this work, after installing two bypass diodes, to create two sub-strings of three cells each.

In order to gain insight into what strategy to follow for meaningful CS current mapping measurements, I-V curves of the PV module were acquired with and without connecting the bypass diodes. The projection system offers the opportunity to set very precise shading levels on individual cells while fully illuminating the remainder, which is something very useful for this investigation. As a first step, the by-pass diodes were disconnected and a sequence of 
100 patterns with increasing levels of shading were applied to one of the cells while the rest are fully illuminated, acquiring the $I-V$ curve of the module for each shading level. This means that for the first $I-V$ curve the cell is completely shaded (all pixels dark for this cell) while the $100^{\text {th }} I-V$ curve is the same in all cases, as there is no shading at all. The $I-V$ curves for the module when each of cells 3 (large 'defect'), 4 (no defects) and 6 (two small 'defects') are shaded are presented in Figure 6.25. A software was written in LabVIEW in order to acquire the $I-V$ curves using the Keithley 2420 sourcemeter, synchronised with the pattern projections.

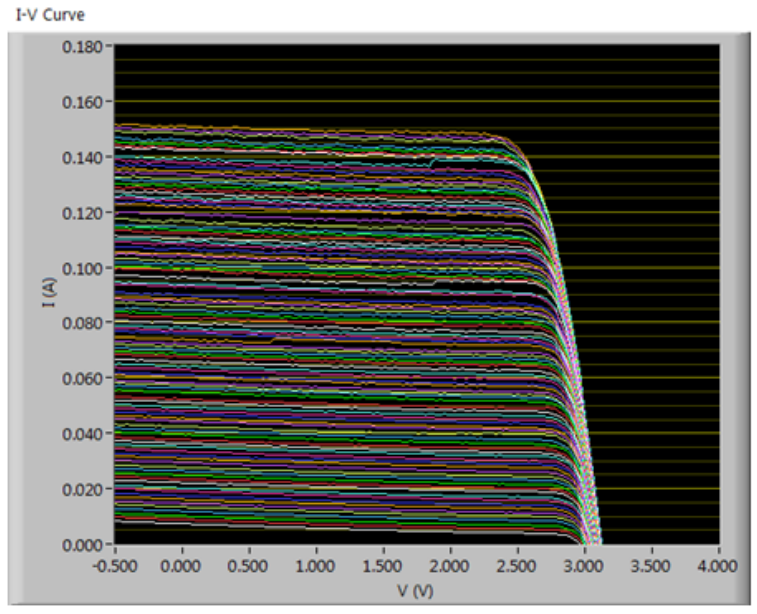

Cell 3

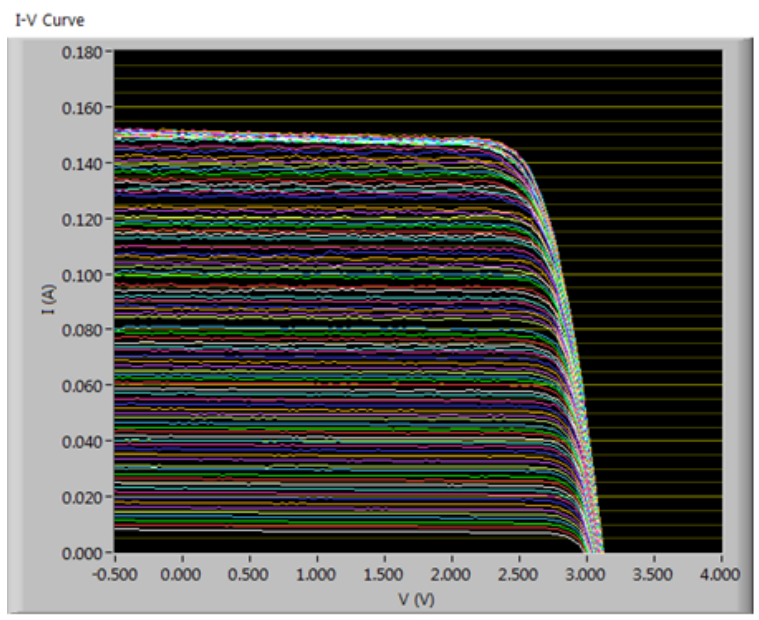

Cell 6

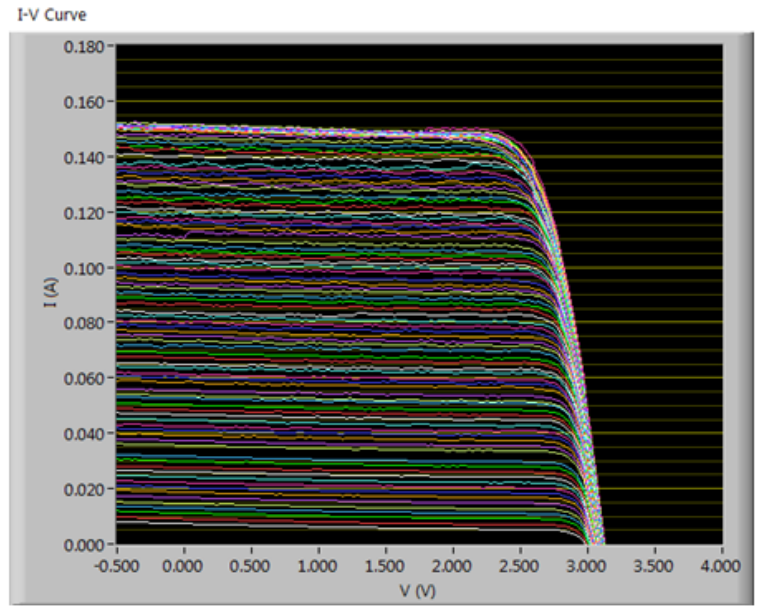

Cell 4

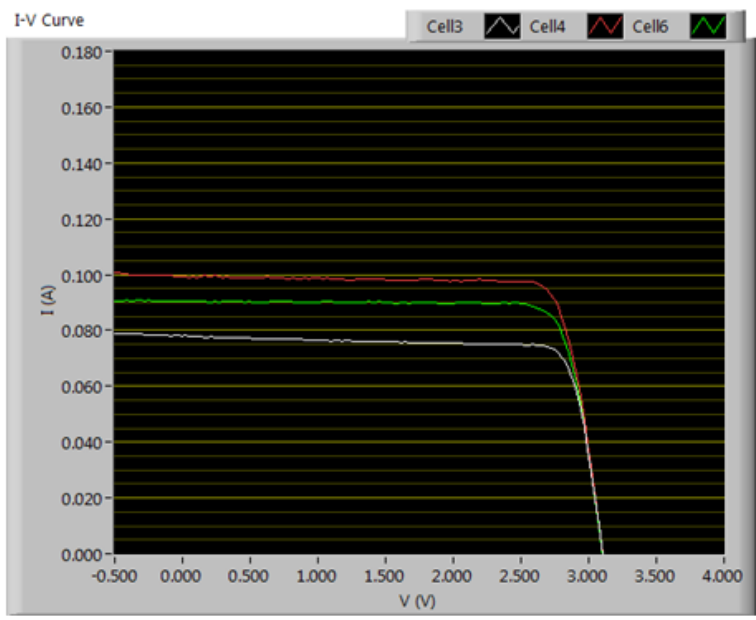

$50 \%$ shading

Figure 6.25. I-V curves of the PV module, acquired by shading one cell each time with different levels of shading. One I-V curve for each cell case with $50 \%$ shading is also included.

The $I-V$ curves of the module for shading of any of the cells exhibit similar behaviour, while for $50 \%$ shading the maximum current depends on the performance of the cell shaded. This is precisely what makes CS current mapping work in the case that all the cells are 
connected in series, which was demonstrated in the previous section. However, when by-pass diodes are installed as shown in Figure 6.24, the $I-V$ curves differ significantly from the case when all the cells are simply series connected. Following the same procedure as before to acquire the same series of $I-V$ curves for shading the same three cells, the results of Figure 6.26 are acquired. It is noteworthy that even with no shading from the projector, the bypass diodes are activated. This is due to the masks that have been applied on some of the cells, resulting in current mismatch, which activates the by-pass diodes.

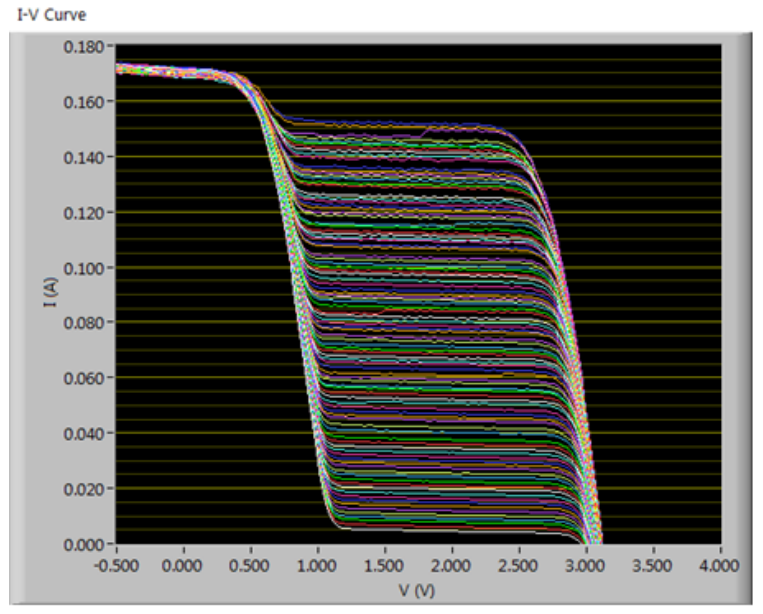

Cell 3

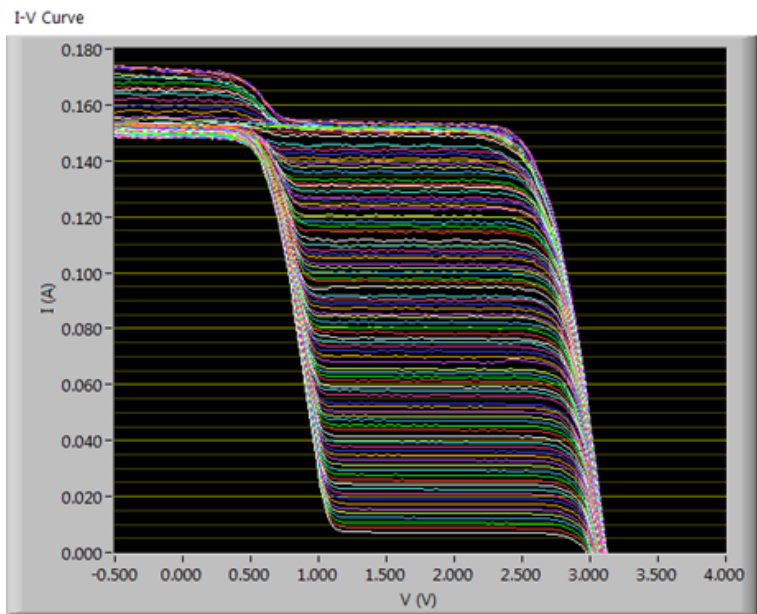

Cell 6

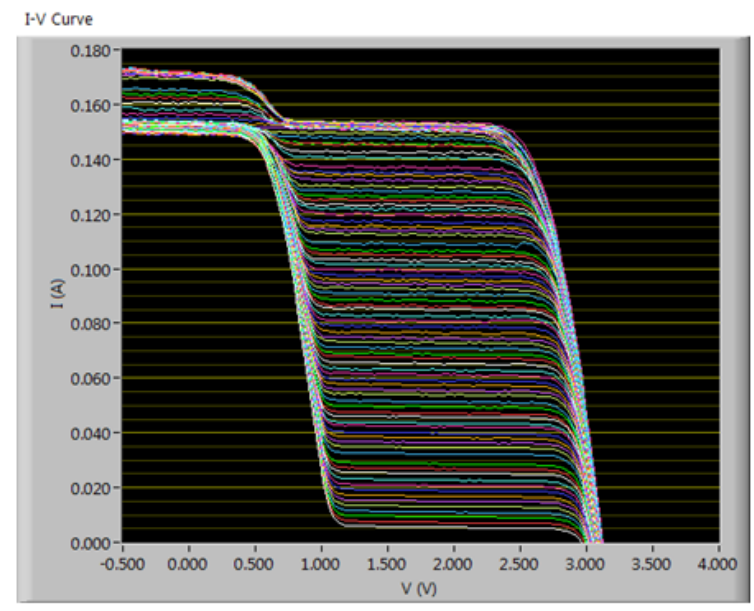

Cell 4

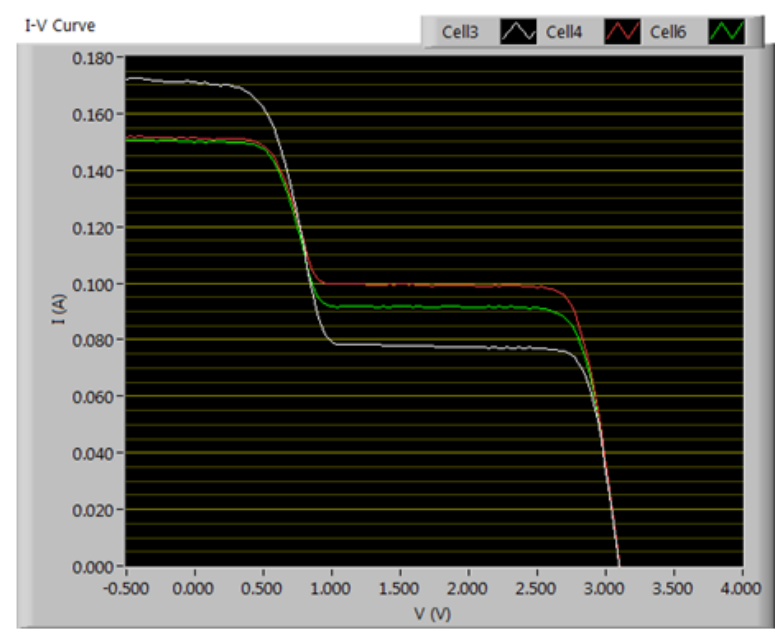

$50 \%$ shading

Figure 6.26. I-V curves of the PV module, acquired by shading one cell each time with different levels of shading, when the PV module has by-pass diodes installed. One I-V curve for each cell case with $50 \%$ shading is also included.

It is clear that the $I-V$ curves differ significantly when by-pass diodes are installed in a PV module. For the application of CS current mapping measurements, a forward voltage bias 
as in the case of the previous section is necessary. Nevertheless, simultaneously with the voltage bias, an additional strategy is also adopted. Since there are three cells in each substring with a by-pass diode, by adjusting the projection on the PV module, one string is completely shaded, while the sub-string that includes the cell that is measured is properly illuminated. The patterns are projected on the cell under test and the rest of the cells of this specific sub-string are fully illuminated. The procedure is illustrated in Figure 6.27.

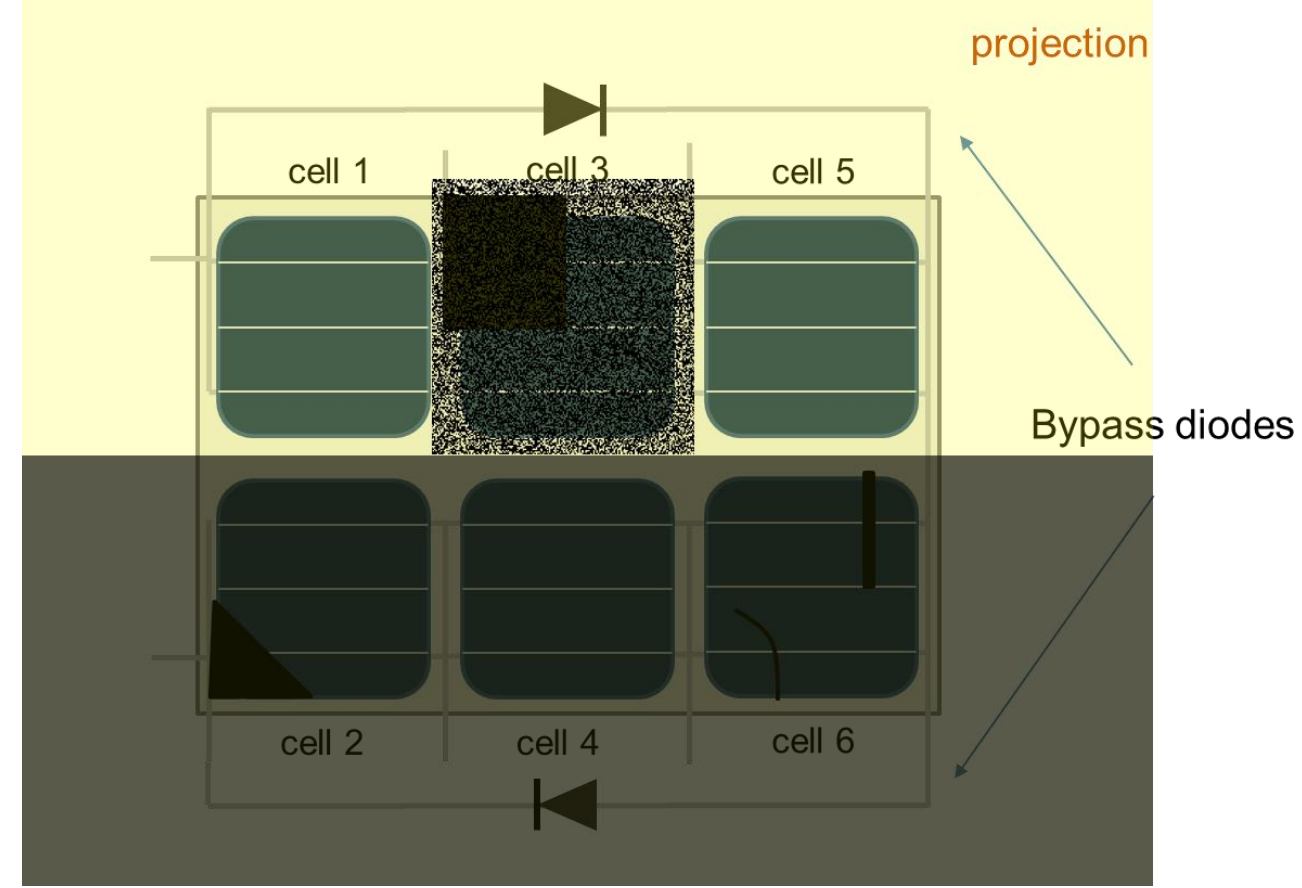

Figure 6.27. The process for CS current mapping measurements of a cell when by-pass diodes are installed in the module. The patterns are projected on the cell under test and the rest of the cells of this specific sub-string are fully illuminated, while the other sub-string is completely shaded.

Following the same procedure for acquiring $I-V$ curves, this time the shading patterns are projected on the cell under test while the rest of the cells of the same sub-string are fully illuminated and the other sub-string is completely shaded (i.e. not illuminated, via software modification of the projector output). The results are presented in Figure 6.28. The influence of the other sub-string is almost negligible and it is barely visible at the bottom of the graphs, where a very low current exists for voltage values higher than $1.1 \mathrm{~V}$. If this voltage region is not considered, the I-V curves resemble those that would be acquired from a 3-cell mini module, with a small voltage drop. This is extremely convenient for the application of compressive sampling, since the current measured at the contacts of the PV module is only 
influenced by the limiting cell (measured cell) of the illuminated sub-string. This is clear when the $I-V$ curves for $50 \%$ shading with this procedure for the three measured cells are displayed in the same graph. It is apparent that the current of the $I-V$ in this instance depends on the performance of the cell of interest.

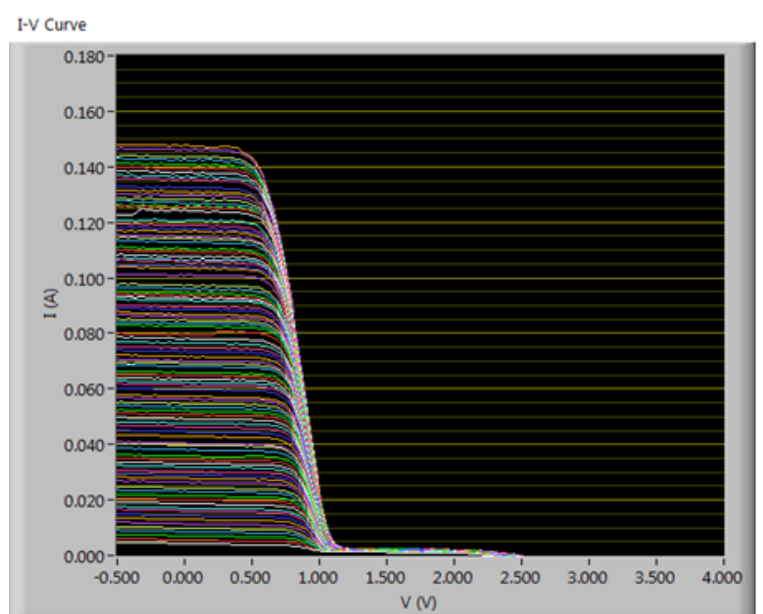

Cell 3

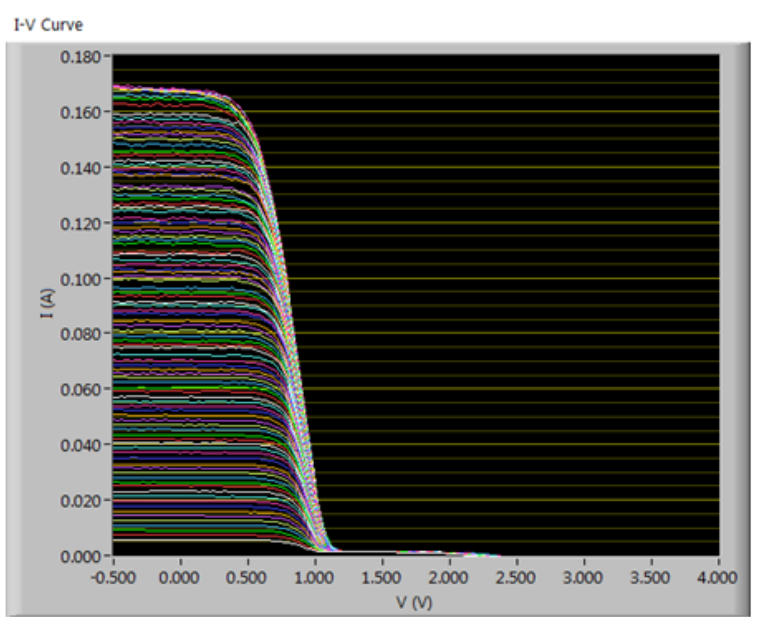

Cell 6

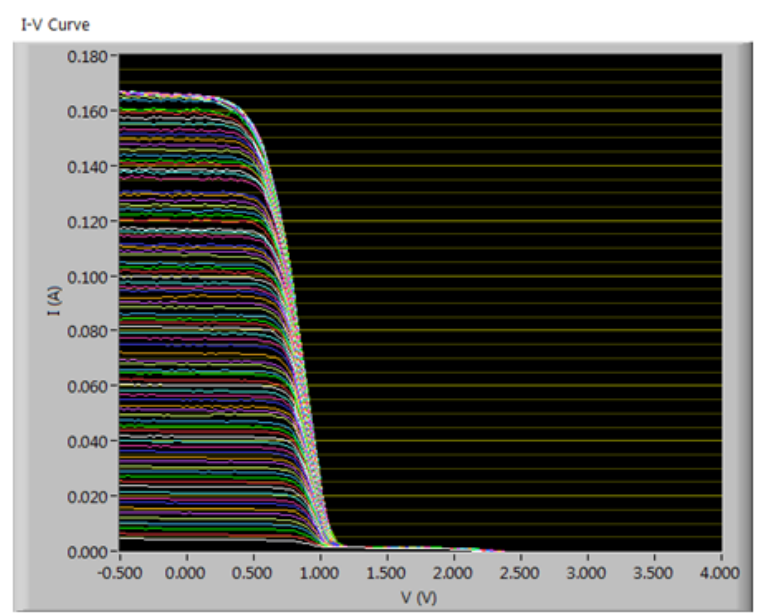

Cell 4

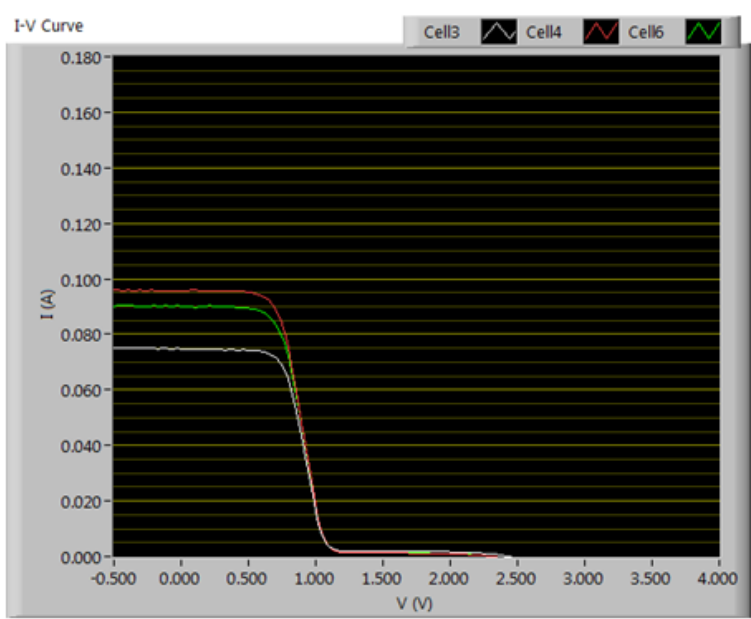

$50 \%$ shading

Figure 6.28. I-V curves of the PV module, acquired by shading one cell each time with different levels of shading, while completely shading the other string, when the PV module has by-pass diodes installed. One I-V curve for each cell case with 50\% shading is also included.

This above strategy where the sub-string not containing the cell under test is shaded is now used for CS current mapping. A forward voltage bias of $0.6 \mathrm{~V}$ is applied during measurements. This value was selected by considering the I-V curves of Figure 6.28. The illuminated cells of the module produce a voltage of around $1 \mathrm{~V}$, hence, by choosing a forward bias of $0.6 \mathrm{~V}$ the cell under test is operating very close to short-circuit conditions. This 
approach also shows that by acquiring the above $I-V$ curves before measurements, one can reveal which cell underperforms. An initial diagnostic test can be set before CS current mapping measurements, where I-V curves are acquired with the same shading level for each cell, following the above shading strategy for disabling cell sub-strings. Such tests are implemented in seconds, since this is the time required to acquire an $I-V$ curve. This procedure can reveal which cells underperform, the cells can be sorted depending on their performance, the correct forward voltage bias levels for the CS current mapping procedure can be chosen. More importantly, with this procedure the correct sparsity levels for the sensing matrices can be determined, to ensure that the patterns will shade the cells to a lower level than the output of the worst performing cell.

Following the procedure of Figure 6.27 for all cells of the mini module with the by-pass diodes installed, the current map shown in Figure 6.29 is acquired. The reconstructed current map for an undersampling level of 50\% (30000 measurements, 60000 pixels) is presented. In the same figure, a photograph of the module during measurements is also presented. The results are very similar to the case of the measurements for the mini module before installing by-pass diodes. The noise is slightly increased, since the average measurement SNR is further reduces, this time being approximately 700 . Nevertheless, meaningful measurements are acquired and this demonstrates the first time that current mapping of a PV module with bypass diodes has been achieved, even with this low resolution.
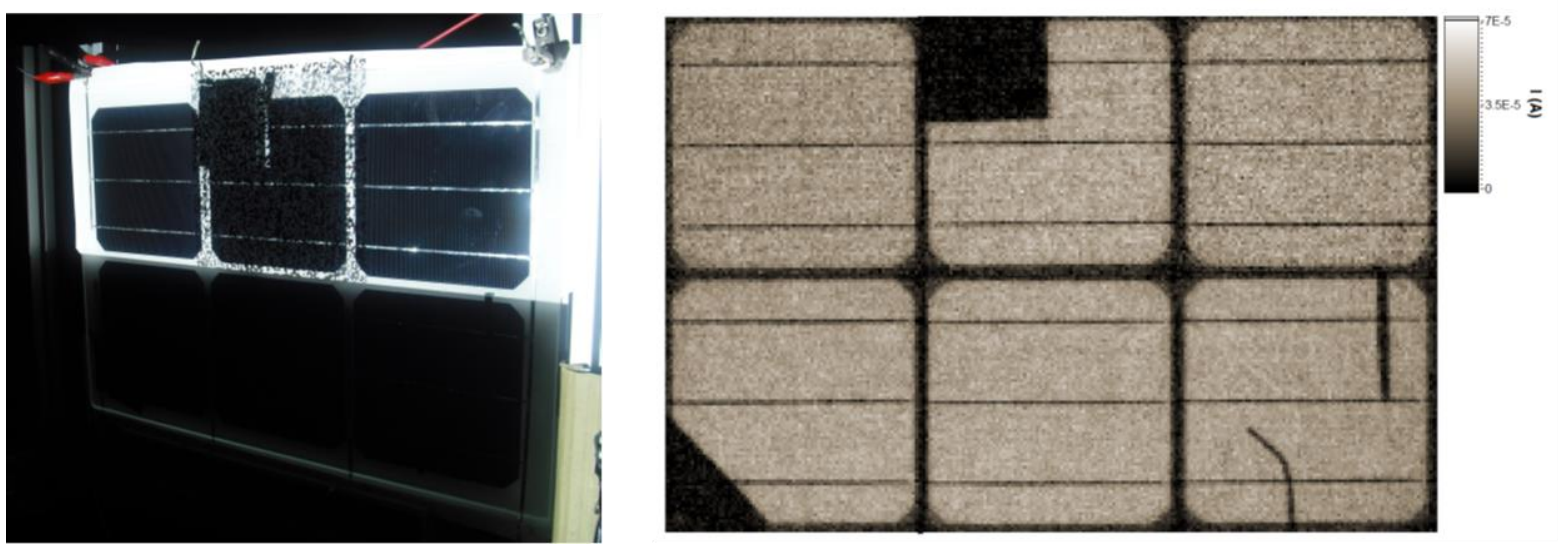

Figure 6.29. On the left, a photograph of the PV module with the attached mask, during measurement when bypass diodes are included. On the right, a CS current map of the PV module with by-pass diodes, with 50\% undersampling. 


\subsubsection{Practical Considerations and Discussion}

The current proof of concept system is based on a commercial projector and as a result, there are several challenges that have already been mentioned and will be summarized in this section. The SNR and the resolution levels achieved are adequate for this proof of concept investigation, but several improvements are possible in order for a DLP projection system to achieve higher measurement accuracy. Considering possible solutions and improvements for minimizing mitigating the issues, the properties of a dedicated projection based system for CS current mapping can be defined.

There is no direct control of the internal DMD kit of the commercial projector. Even after the removal of the colour wheel, the DMD is still switching independently, creating significant variations of intensity. By adjusting the correct settings (brightness, colour balance, contrast) through the user interface of the projector and by applying filters the signal stability was significantly enhanced. In a custom system where the DMD would be directly controlled, similar to the small area system of the previous chapter, the signal can be made very stable. This will reduce noise levels and increase both the speed and accuracy of measurements, achieving a signal to noise ratio similar to that of the small area optical system.

As presented earlier, there are long term instabilities of illumination intensity of around $\pm 3.5 \%$ of the average value. This variation can affect compressive sampling and for this reason the monitoring of illumination intensity with the photodiode is significant, in order for each measurement to be normalised. A custom future projector system could rely on a high power laser source, which would provide more stability. An approach similar to high end cinema DLP projectors could be adopted, which can boost irradiance levels by at least a factor of 10 [208][205]. 3 DMD chips can be used, with each having its own independent light source. As a result, no colour wheel is used, with the system being more efficient and irradiance intensity being far more stable. A reference measurement with a photodiode for irradiance monitoring can still be applied for normalising readings and increasing measurement accuracy. A single wavelength light source will also set a specific penetration depth of light into the PV device under test. The current white light of the projector lamp has a very broad spectrum for such an investigation. The selection of a specific single wavelength light source, in the infra-red region for measuring silicon samples, will allow probing of the bulk region of silicon PV devices, as in the case of the small area setup.

Before starting any measurements with the current setup, there is a period of time of around 15 minutes that a white image is projected on the sample, with all the pixels at the 
"on" state, without realising any measurement. This is done because it was observed that the temperature of the projection lens influenced its focal length. During the initial system setup, after a lens warm-up period, the projection was focused on the sample plane and this focus setting was kept throughout measurements, with frequent testing of sharpness of projection. Regarding the temperature of the PV device, due to the low irradiance levels any increase in temperature will be negligible. Nevertheless, in the case of forward voltage bias or when a cell is significantly shaded, the temperature increase may not be negligible anymore. Consequently, temperature monitoring should be considered in a future implementation, especially for the cell under measurement.

Irradiance uniformity of the projection on the sample plane is also important. Not all the projection area is used but only a central part, nevertheless, a non-uniformity of approximately $10 \%$ still exists. A calibration can be considered in a future system to correct out this effect. However, non-linearity of samples for low irradiance may also add some error to measurements. On the other hand, in the case that a high power projection setup is utilised, similar to cinema projection systems, a configuration similar to that of solar simulators can be adopted, with a long projection distance. This would reduce irradiance non-uniformities to acceptable levels.

It is observed that from the PV cell to the PV module case of CS current mapping, the measurement SNR is reduced. Even if a custom projector system is realised which will have increased measurement accuracy and higher SNR, this decrease from cell to module case will still be present. This is due to the influence from the rest of the cells of the module, which are fully illuminated. It was already observed in previous work regarding current mapping of PV modules that the features of individual cells become less sharp when point by point current mapping is applied at a module level [78]. In our case, due to the global nature of each measurement, this results in a slightly increased measurement noise. Nonetheless, increasing the $S N R$ to a higher level in a future custom projection system, this issue would become less pronounced and even negligible, depending on the SNR achieved.

Regardless of the limitations mentioned above, the projector based system offers some special features that can be utilised for PV characterisation generally and not only for current mapping. The ease of application of shading patterns and the accuracy of the level of shading settings can provide a very useful tool for investigation of shading effects on PV modules. This is demonstrated specifically in section 6.4.4 where $I-V$ curves of the module with the by-pass diodes were acquired for several different shading conditions. Although in our case the aim is to apply suitable shading patterns for successful CS current mapping, a projector system 
can be an extremely useful tool for studying the effects of shading on a commercial module's overall performance. In addition, it is clear that by applying the suitable shading strategies on a module, the performance of individual cells can be acquired by just measuring the I-V curve of the PV module. There are several methods in the literature that utilise mechanical shading for this purpose [209][210]. A projector system would be an extremely practical tool for the application of shading strategies for such method, increasing measurement speed and shading accuracy.

\subsection{Conclusion}

A simple DLP projection based system has been developed that utilizes CS for current mapping of PV devices. The system has been used to demonstrate that a scale up of the small area optical CS current mapping system presented in the previous chapter is possible. The issues and limitations of the DLP projector as a light source are investigated and the necessary adjustments are made in order to make the system suitable for current mapping measurements.

Experimental results demonstrate that a DLP projector based CS current mapping system is feasible, since CS current mapping is successfully applied to PV cells and also to PV modules. Current maps of individual PV cells in encapsulated modules have been acquired, something that is extremely difficult to achieve with conventional LBIC systems. This is the first time that current mapping of a PV module with by-pass diodes has been successfully demonstrated. Specific shading strategies were developed for this purpose in order to isolate the cell under test. It is also demonstrated that such shading strategies can also be applied and the I-V curve of the module can be acquired, in order to detect if any cell in a module is underperforming. This diagnosis process takes seconds using the DLP projector and can sort the cells of a module in order of performance. This can also be useful in order to determine the sensing matrix sparsity necessary for CS current mapping measurements.

Due to the application of compressive sampling, current maps are acquired even if the SNR levels are so low that a point by point scan is not possible. Using CS, significantly fewer measurements are required to produce the current maps than a point by point scan would 
need. On the other hand, this primitive prototype system approach cannot achieve very high accuracy and optical resolution by using such a commercial DLP projector. This is mainly because of the high noise levels and the low irradiance produced by this system. A custom system will provide more control on the DMD chip and will be able to reduce temporal variations of light intensity. This would decrease noise levels and provide higher accuracy. Recent developments of DLP high power multiple wavelength cinema laser projectors have been identified that would allow the use of high irradiance levels, close to standard test conditions for PV modules. The necessary properties that such a projector based system would need to possess are determined and discussed in this work, making such an implementation a realistic solution for a new approach for PV module spatial characterisation. 


\section{Chapter 7}

\section{Conclusions}

\subsection{Conclusions}

The main goal of this work was to combine current mapping measurements of PV devices with compressive sampling, in order to develop a novel current mapping method for faster, more accurate and less expensive measurements than provided by the current stateof-the-art. This work presents the first application of the compressed sensing sampling theory to PV characterisation. This approach was investigated through three different phases:

- Electrical simulations of CS current mapping were implemented using a spatially resolved model previously developed in CREST, simulating the whole measurement procedure.

- A small area optical CS current mapping system for PV devices, built by the author at NPL. This setup was based on a DMD chip for applying compressive sampling and it is realised in a very simple and straightforward way, requiring no moving parts, complicated optical elements or lock-in methods.

- A projector based CS current mapping system built at CREST. This setup was based on a commercial DLP projector and its aim was to demonstrate that a scale up of the small area system for current mapping of PV modules is possible. 
The following sections summarise the findings and draw the main conclusions within these three areas. Recommendations for further research and future prospects resulting from this work are given in the last section.

\subsubsection{Spatially Resolved Electrical Simulations of Compressed Sensing Current Mapping}

Before realising any experimental implementations, in order to theoretically confirm the feasibility of the method and evaluate its performance, simulations of CS sensing current mapping were implemented. The spatially resolved electrical simulations were realised using the PVONA toolset for solving the spatially resolved model of solar cells, based on the one diode model. Simulated cases of both c-Si and thin film devices were investigated, showing that the performance of the method is the same, regardless of the features a sample may contain.

Through the simulations it was demonstrated that reliable current maps with compressive sampling can be acquired with fewer than half of the measurements that a point by point scan would need. In addition to simulating individual PV devices, a simulation for the application of this method to PV modules was realised. Through the series of simulations the feasibility of the method was validated and the performance with different levels of undersampling was investigated.

A significant advantage of the simulation process is the absence of noise and the controlled environment it provides, excluding measurement artefacts. For this reason, simulation was selected as an appropriate way to achieve a reliable evaluation of different CS reconstruction algorithms prior to experimental implementations. Several different reconstruction algorithms were tested, in order to find the most suitable for this application. The results showed that the $\ell 1$ minimisation algorithm had the best performance for the simulated case, both in terms of speed and reconstruction accuracy. For this reason, it was the preferred algorithm throughout this work, along with the OMP algorithm.

Different types of sensing matrices for compressive sampling were also tested. These included different types of random matrices and structured Hadamard matrices. It was shown that all random matrices have the same performance, slightly better than the Hadamard matrices. Hence, in this work uniformly distributed random binary matrices are used for compressive sampling, since they are also easy to implement and they meet the incoherence criterion when the DCT transform is used for reconstruction. 
Controlled levels of noise were added to the experimental procedure to test the noise tolerance of the method. Although the performance of CS current mapping can be affected by noise, the noise levels are usually low during compressive sampling, as the signal is enhanced since a large area of the sample is illuminated. This was clearer during the experimental procedure.

\subsubsection{Small Area Compressed Sensing Current Mapping system}

After the realisation of the electrical simulations which theoretically confirmed the feasibility of the method, a CS current mapping optical setup was implemented at NPL. This measurement system was built based on a DMD chip, with the aim of achieving an alternative approach for current mapping of PV devices. The system was designed so that it mitigates some drawbacks of conventional LBIC systems; low measurement speed, complicated layouts and expensive equipment necessary for accurate measurements. The result was the implementation of a very simple and compact measurement setup, with no moving parts and a small number of optical elements. In addition, the need for the use of lock-in techniques for increased measurement accuracy is diminished, reducing the system cost significantly. Apart from the above properties, the system developed in this work is the first reported implementation of a reliable current mapping system for PV devices that utilises compressive sampling. By using different optical elements to reduce the size of the experimental layout, it was demonstrated that a compact, inexpensive instrument can be built which can be commercialised as a PV characterisation tool.

The experimental results with this setup showed that CS current mapping yields reliable results with fewer acquired measurements than a point by point scan. Reliable current maps with undersampling levels of at least $50 \%$ were acquired with compressive sampling, which can lead to a significant reduction of measurement time. Due to control software restrictions, the system achieved a sampling rate of only 10 measurement per second. Considering that fewer measurements are acquired, the overall measurement time is similar with a modern, state of the art LBIC system. Nevertheless, a much higher sampling rate is achievable, by accessing the internal memory of the DMD's memory board to store the sensing matrix prior to measurements. This can increase sampling rate by even an order of magnitude compared to the latest LBIC systems, depending of course on the sample response. Combined with the fewer measurements required, the spatial characterisation method developed in this work shows great potential for reducing current mapping measurement time of PV devices. 
Measurements of both thin film and crystalline silicon PV samples were implemented. It was shown that for small area samples with low noise levels, this setup can apply both point by point scans and compressive sampling. When noise levels are increased, as they are for the large mc-Si sample, a point by point scan failed due to increased noise. However, compressive sampling still provides reliable current mapping results, significantly enhancing measurement SNR levels. This shows that using this setup a different sampling strategy can be selected every time, depending on the type of the sample, the required resolution, the SNR levels and the time constraints. Thus, the current mapping measurement instrument developed is not only cost effective but also very versatile.

Since both a raster scan and compressive sampling can be applied with the small area CS setup, a direct pixel by pixel comparison of current maps acquired with each method was possible. This allowed an experimental, quantitative evaluation of CS current mapping, using two different types of reconstruction algorithms and sensing matrices with different levels of sparsity. The results showed that the choice of reconstruction algorithm is important since different algorithms can have different performance, depending on the sample, the noise levels and the sensing matrix sparsity. It was shown that sensing matrix sparsity is a compromise between high SNR and reconstruction performance, which is in accordance with other studies in the literature. The majority, if not all, comparisons of CS with point by point sampling reported in the literature are realised using simulations, whereas this work has directly demonstrated the technique with real measurements. This optical setup allows the investigation of different CS components such as algorithms, sensing matrices and transforms in a real measurement environment. As a result, such an instrument can be useful not only for PV characterisation, but also for the CS metrology community.

\subsubsection{Scalability Compressed Sensing Current Mapping}

The final step of this work was to investigate if a scale up for this method is possible, in order to apply it to large samples and specifically PV modules. This was studied utilising a commercial DLP projector. Since DLP projectors are based on a DMD chip, such a system provides a perfect means to project the required patterns onto the sample under measurement, in order to apply compressive sampling. A DLP projection based current mapping system was built at CREST and measurements with wafer based c-Si cells and a custom PV module were implemented. The feasibility of CS current mapping with a DLP projector for both single PV cells and PV modules was confirmed as a result of these 
measurements. Similar to the small area CS current mapping system, this setup included no moving parts, the cost was rather low and its implementation was simple. Since the commercial DLP projector used is not intended for use as a light source for metrology applications, there were several inherent challenges that had to be overcome. Temporal spectral variations, short and long term stability of irradiance intensity and non-uniformity of illuminated area were some of the problems that had to be tackled and mitigated. Several changes applied resulted in a system that could apply CS current mapping measurements that confirmed the feasibility of this upscale approach. In addition, these alterations indicated the properties that a custom projection system intended for CS current mapping should possess.

The most significant achievement with this system was the acquisition of current maps of PV modules, using compressive sampling. Current maps of individual PV cells in encapsulated modules can be acquired, something that is extremely difficult to achieve with conventional LBIC systems. In fact, this is the first time that current mapping of a PV module with by-pass diodes has been successfully delivered. By application of specific sampling strategies depending on the PV module architecture, individual cells of any PV module can be assessed with a projection based current mapping system. An additional useful feature is that the $I-V$ curves of the module with specific shading strategies can be acquired, in order to detect if any cell in a module is underperforming. This process can sort the individual cells of a module depending on performance and define the required sparsity of the sensing matrices that should be used for a specific module. The suitable sensing matrix sparsity ensures that the cell under test is the limiting cell of the module, or the sub-string of cells that is being measured.

The compressive sampling principles for current mapping were successfully applied, acquiring current maps with fewer measurements than required by a point by point scan. Current maps can be acquired even if the SNR levels are so low that a point by point scan is not possible. Even if the sampling rate was rather low and not such a high resolution was achieved, this proof of concept study was successful and it has the potential to become a reference point for future applications of projection technology on PV characterisation. 


\subsection{Further research and future prospects}

This work developed and investigated thoroughly the CS current mapping method through simulations and two different experimental layouts, but also identified aspects that would benefit from further research. The main aspect with room for improvement concerns the mathematical part of the method. Although several algorithms and sensing matrices were investigated, the CS mathematical field is vast and new reconstruction algorithms and procedures are constantly reported in the literature. The adoption of some of the latest algorithms may significantly improve the performance of the method, further reducing measurement time. In addition, large-scale CS current mapping with megapixel resolution can become possible, although complex mathematical models have to be integrated into the experimental procedure. Such an approach can be extremely useful in the case of PV module characterisation. High resolution CS current mapping can finally offer a realistic solution for reliable current mapping of PV modules, where measurements for a large module last hours instead of days.

The small area CS current mapping setup could be commercialised as a low cost PV current mapping instrument, with only a few changes. These are mainly the irradiance uniformity on the sample, the use of shorter focal length optical elements to decrease the size of the setup and optimisation of the DMD control software. Along with the development of a standard CS procedure suitable for this application, including sensing matrices, transforms and reconstruction algorithms integrated into the software, such an instrument can become a realistic low cost solution for current mapping of small area devices. This would be very useful for research laboratories that work on the development of new solar cell materials and designs, where the samples are generally smaller than $1 \mathrm{~cm}$ by $1 \mathrm{~cm}$.

Furthermore, an imaging microscope objective lens can be integrated in the small area CS setup, leading to even higher optical resolution with a very small additional cost. In addition, using this approach and considering CS microscopy applications [194][189], this setup can be transformed to a more broad spatial characterisation instrument, since a potential imaging microscope objective lens can also be used as a photon collection optic. The simultaneous application of different characterisation techniques such as current mapping, photoluminescence and spatial Raman spectroscopy is theoretically possible, with the addition of the necessary detectors and optics. The application of CS to most of these characterisation techniques has already been reported in the literature and the development of such a CS instrument for PV characterisation is just a matter of time. The design and 
approach of any such setup will inevitably use the principles and main design of the optical system demonstrated in this work.

Regarding the DLP projector system, after the proof of concept system developed and demonstrated in this work, the next step would be a custom CS DLP system, with a design dedicated for current mapping of PV modules. Such a system cannot be based on a commercial projector, but should be built from components, considering the findings of this work about the properties that such a system should possess. With the recent advances in cinema high power laser projectors, it is possible that 1 sun illumination on a large PV module can be achieved. This can even lead to the acquisition of current maps of PV modules under standard conditions, using a projection system and the methodology of this work. DLP projection based solar simulators can be developed in the future, where individual cells can be sensed even after encapsulation as modules.

The huge flexibility of the DLP projector system in creating shading patterns demonstrated in this work can help in a more thorough evaluation of shading effects in a PV module, helping to validate and further develop establish models. In addition, assessing individual cells using known methods can become faster when projector technology is used instead of mechanical shading. The accuracy and variety of controlled shading patterns that a projector can apply to a PV module, combined with the acquired I-V curves of the module can lead to the evaluation of specific parameters of individual cells of a module. As a result of this potential capability, new models can be developed for determining parameters of individual cells, using shaded $I-V$ curves of the module, even when by-pass diodes are included. This will greatly help the understanding of cell ageing mismatch effects on PV module performance and can support research into more reliable PV products.

Overall, the contribution of this work reflects not only current mapping of PV devices, but also on PV characterisation as a whole, both for PV cells and modules. There is a high possibility that new characterisation methods are developed based on this work, some of them already mentioned above. In addition, through this first application of the CS theory on PV characterisation, it is shown that CS can be a very useful sampling technique that can have further applications within the metrology community. 


\section{Appendix}

\section{Reconstruction algorithms for CS used in this work}

\section{Reconstruction based on $\mathrm{\ell} 1$ norm minimisation}

As described in chapter 3 [cross ref], After applying compressive sampling, the reconstruction algorithm must take the $M$ measurements in the vector $y$, the random binary matrix $\Phi$, the basis $\psi$ (transform) and reconstruct the $N$-length signal $x$ or, equivalently, its sparse coefficient vector $\boldsymbol{\alpha}$, as $\boldsymbol{x}=\Psi \boldsymbol{\alpha}$ and $\boldsymbol{y}=\Phi \boldsymbol{x}=\Phi \Psi \boldsymbol{\alpha}$. When the $\ell 1$ minimisation algorithm is used, the solution to the underdetermined problem is the $\boldsymbol{x}$ vector (or more precisely the $\alpha$ vector) with the minimum $\ell_{1}$ norm [174]:

$$
\widehat{\boldsymbol{x}}=\operatorname{argmin}\|\boldsymbol{x}\|_{1} \text { subject to } \Phi \widehat{\boldsymbol{x}}=\boldsymbol{y}
$$

Or

$$
\widehat{\boldsymbol{\alpha}}=\operatorname{argmin}\|\boldsymbol{\alpha}\|_{1} \text { subject to } \Phi \Psi \widehat{\boldsymbol{\alpha}}=\boldsymbol{y}
$$

Where the $\ell 1$ norm is given by

$$
\|x\|_{1}=\sum_{i=1}^{n}\left|x_{i}\right|
$$

In the case where measurement noise is included, equation (1) can be written as [211]:

$$
\widehat{\boldsymbol{x}}=\operatorname{argmin}\|\boldsymbol{x}\|_{1} \text { subject to }\|\Phi \widehat{\boldsymbol{x}}-\boldsymbol{y}\|_{2} \leq \varepsilon
$$

Where $\varepsilon$ is a tolerance parameter. This algorithm mentioned as $\ell 1$ denoise, was used in chapter 4 [cross ref] without improving reconstruction results where noise was included. Equation (1) can be rewritten as

$$
\min _{x, u} \sum_{i=1}^{n}\left|\boldsymbol{u}_{i}\right|
$$

With

$$
\begin{gathered}
x_{i}-u_{i} \leq 0, i=1,2, \ldots, n \\
-x_{i}-u_{i} \leq 0, i=1,2, \ldots, n \\
A \boldsymbol{x}=\boldsymbol{y}
\end{gathered}
$$


This last form is a standard linear programming problem known as basis pursuit [212][213]. A relatively simple primal-dual algorithm for linear programming is described in [213]. As the $\ell 1$ primal dual implementation included in the $\ell 1$ magic package is mostly used in this work, the primal dual algorithm is briefly described here. The standard form linear program is

$$
\begin{array}{ll}
\min _{z}\left\langle c_{0}, z\right\rangle \text { subject to } & A_{0} z=b \\
& f_{i}(z) \leq 0
\end{array}
$$

Where $z \in \mathbb{R}^{N}$ is the search vector, be $R^{K}, A_{0}$ is a $K \times N$ matrix and $f_{i}, i=1,2, \ldots, m$ is the linear functional

$$
f_{i}(\mathrm{z})=\left\langle c_{i}, z\right\rangle+d_{i}
$$

Where $c_{i} \in \mathbb{R}^{N}$ and $d_{i} \in \mathbb{R}$ are constants. At the optimal point $z^{*}$ there will exist dual vectors $v^{*} \in \mathbb{R}^{K}, \lambda \in R^{m}$, such that the Karush-Kuhn-Tucker ${ }^{1}$ conditions are satisfied.

$$
\begin{gathered}
c_{0}+A_{0}^{T} v^{*}+\sum_{\iota} \lambda_{i}^{*} c_{i}=0 \\
A_{0} z^{*}=b \begin{array}{r}
\lambda_{i}^{*} f_{i}\left(z^{*}\right)=0, i=1,2, \ldots, m \\
f_{i}\left(z^{*}\right) \leq 0, i=1,2, \ldots, m
\end{array}
\end{gathered}
$$

The primal dual algorithm solves this system of nonlinear equations and provides the optimal $z^{*}$. The classical Newton method is used for this procedure. The system is linearised and solved at an interior point $\left(z^{k}, v^{k}, \lambda^{k}\right)$, with the interior point constraints of $f_{i}\left(z^{k}\right)<0$ and $\lambda^{k}>0$. The step of the iterative method has to be modified so that we remain in the interior. In practice, a slackness parameter $\tau$ is introduced and the slackness condition $\lambda_{i} f_{i}=$ 0 is relaxed to 


$$
\lambda_{i}^{k} f_{i}\left(z^{k}\right)=-1 / \tau^{\kappa}
$$

$T \tau$ is increased after each newton iteration. The primal, dual and central residuals that quantify how close how close a point $(z, \lambda, v)$ it to satisfy (8) with the new relaxed conditions are given by:

$$
\begin{gathered}
r_{d u a l}=c_{0}+A_{0}^{T} v+\sum_{i} \lambda_{i} c_{i} \\
r_{c e n t}=-\Lambda f-(1 / \tau) \mathbf{1} \\
r_{p r i}=A_{0} z-b
\end{gathered}
$$

Where $\Lambda$ is the diagonal matrix $(\Lambda)_{i i}=\lambda_{i}$, and $f=\left(f_{1}(z), \ldots, f_{m}(z)\right)^{T}$. Assuming the current point is $(z, v, \lambda)$, the aim is to find the $\operatorname{step}(\Delta z, \Delta v, \Delta \lambda)$ such that:

$$
r_{\tau}(z+\Delta z, v+\Delta v, \lambda+\Delta \lambda)=0
$$

The Taylor expansion of (11) around the point $(z, v, \lambda)$,

$$
r_{\tau}(z+\Delta z, v+\Delta v, \lambda+\Delta \lambda) \approx r_{\tau}(z, v, \lambda)+J_{r_{\tau}}(z, v, \lambda)\left(\begin{array}{l}
\Delta z \\
\Delta v \\
\Delta \lambda
\end{array}\right)
$$

Where $J_{r_{\tau}}(z, v, \lambda)$ is the Jacobian of $r_{\tau}$. The result is the system

$$
\left(\begin{array}{ccc}
0 & A_{0}^{T} & C^{T} \\
-\Lambda C & 0 & -F \\
A_{0}^{T} & 0 & 0
\end{array}\right)\left(\begin{array}{c}
\Delta z \\
\Delta v \\
\Delta \lambda
\end{array}\right)=-\left(\begin{array}{c}
c_{0}+A_{0}^{T} v+\sum_{i} \lambda_{i} c_{i} \\
-\Lambda f-(1 / \tau) \mathbf{1} \\
A_{0} z-b
\end{array}\right)
$$

Where $\mathrm{C}$ is a $\mathrm{m} \times \mathrm{N}$ matrix with $c_{i}^{T}$ as rows and $\mathrm{F}$ is a diagonal matrix with $(F)_{i i}=f_{i}(z)$. $\Delta \lambda$ can be eliminated using:

$$
\Delta \lambda=-\Lambda F^{-1} C \Delta z-\lambda-(1 / \tau) f^{-1}
$$

Resulting in the system: 


$$
\left(\begin{array}{cc}
-C^{T} F^{-1} \Lambda C & A_{0}^{T} \\
A_{0} & 0
\end{array}\right)\left(\begin{array}{c}
\Delta z \\
\Delta v
\end{array}\right)=\left(\begin{array}{c}
-c_{0}+\left(\frac{1}{\tau}\right) C^{T} f^{-1}-A_{0}^{T} v \\
b-A_{0} z
\end{array}\right)
$$

With $(\Delta \mathrm{z}, \Delta \mathrm{v}, \Delta \lambda)$ being the step. Assuming a step length of $\mathrm{s}$, with $0<s<1$, the step has to satisfy the two criteria:

1. $z+s \Delta z$ and $\lambda+\Delta \lambda$ are in the interior, which means $f_{i}(z+s \Delta z)<0, \lambda_{i}>0$ stand for all $i$.

2. The norm of the residuals has decreased sufficiently:

$$
\left\|r_{\tau}(z+s \Delta z, v+s \Delta v, \lambda+s \Delta \lambda)\right\|_{2} \leq(1-\alpha s) \cdot\left\|r_{\tau}(z, v, \lambda)\right\|_{2}
$$

Where $\alpha$ is a user specified tolerance constant. When $r_{\text {dual }}$ and $r_{\text {primal }}$ are small, $n=-f^{T} \lambda$ is an approximation of how close a certain point $(z, v, \lambda)$ is to being the optimal, since $\left\langle c_{0}, z\right\rangle-\left\langle c_{0}, z^{*}\right\rangle \approx n$. The primal dual algorithm repeats the Newton iterations described above until $\mathrm{n}$ has decreased below a set tolerance.

Other options for solving the $\ell 1$ optimisation problem also exist, such as the Dantzig selector algorithm [179] which is included in $\ell 1$ magic toolbox in MatLab [214], or the spectral projected-gradient (SPG) algorithm [178][215], which are briefly used in chapter 4 [cross ref], but not described here.

\section{Reconstruction based on Total Variation norm minimisation}

Similarly with $\mathrm{e} 1$ optimisation, the total variation norm minimisation is briefly used in chapter 4 [cross ref]. Since the signal we are measuring is a 2D image, the discrete gradient at every point can be calculated. With $\boldsymbol{x}_{i j}$ being the pixel in the $i$ row and $j$ column of an $n \times$ $n$ image $\boldsymbol{x}$ the operators of the discrete gradient of the image $\mathbf{x}$ can be defined:

$$
\begin{gathered}
D_{h ; i j} x=\left\{\begin{array}{ll}
x_{i+1, j}-x_{i j} & i<n \\
0 & i=0
\end{array} \quad D_{v ; i j} x= \begin{cases}x_{i, j+1}-x_{i j} & i<n \\
0 & i=0\end{cases} \right. \\
D_{i j} x=\left(\begin{array}{l}
D_{h ; i j} x \\
D_{v ; i j} x
\end{array}\right)
\end{gathered}
$$


The total variation of $\mathbf{x}$ is the sum of magnitudes of this gradient at every point:

$$
\|x\|_{T V}=T V(x)=\sum_{i j} \sqrt{\left(D_{h: i j} x\right)^{2}+\left(D_{v: i j} x\right)^{2}}=\sum_{i j}\left\|D_{i j} x\right\|_{2}
$$

As a result, instead of equation (1), when using TV minimisation the optimisation problem becomes:

$$
\widehat{\boldsymbol{x}}=\operatorname{argmin}\|\boldsymbol{x}\|_{T V} \text { subject to } \Phi \widehat{\boldsymbol{x}}=\boldsymbol{y}
$$

Primal dual algorithms for solving this problem as the one described for $\ell 1$ minimisation exist [216][217]. However, the implementation used in this work included in the $\ell 1$ magic toolbox uses the log-barrier method, more information of which can be found in [213] and it is not described here.

\section{Summary of matching pursuit algorithms}

Matching pursuit is a sparse approximation algorithm which aims at finding the "best matching" representation of data using a specific dictionary $\boldsymbol{D}$ (a "dictionary" could be a transform domain like the Fourier transform). A signal $f$ can be represented as a sum of vectors $\left\{g_{\gamma}\right\}_{\gamma \in \Gamma}, \Gamma$ being the index set and $\left\|g_{\gamma}\right\|=1$. The vectors $\left\{g_{\gamma}\right\}_{\gamma \in \Gamma}$ are called atoms, taken from $\boldsymbol{D}$. An approximation of the signal using $\mathrm{N}$ atoms can be written as

$$
\hat{f}=\sum_{n=1}^{N} a_{n} g_{n}
$$

Where $a_{n}$ are weighing factors for the atoms. This decomposition can be written as

$$
f=\langle f, g\rangle g+r^{(g)}
$$


Which includes the actual signal $f$ as well as the approximation $\langle f, g\rangle g$ using the dictionary $\boldsymbol{D} . r^{(g)}$ is the residual after approximating $f$ by the atoms $g_{n}$. As the residual is orthogonal to $f$, the following equation stands:

$$
\|f\|^{2}=|\langle f, g\rangle|^{2}+\left\|r^{(g)}\right\|^{2}
$$

For achieving the "best" approximation the residual should be minimised, or $|\langle f, g\rangle|^{2}$ should be maximised, given $\|f\|^{2}$ is the actual signal and always the same value. At each iteration a vector is chosen that is "almost best", in the sense that

$$
|\langle f, g\rangle|^{2} \geq \operatorname{asup}\left|\left\langle f, g_{\gamma}\right\rangle\right|
$$

Where sup is the supremum and $\alpha$ is a constant that satisfies $1<a \leq 1$. At each iteration the matching pursuit algorithm will choose the almost best approximation so that the current residual from the dictionary is minimised, or equivalently $|\langle f, g\rangle|^{2}$ is maximised. The $n$th residual is given by

$$
r^{(n)}=\left\langle r^{(n)}, g^{(n)}\right\rangle g^{(n)}+r^{(n+1)}
$$

Resulting in

$$
f=\sum_{i=0}^{n}\left\langle r^{(i)}, g^{(i)}\right\rangle g^{(i)}+r^{(n+1)}
$$

The Orthogonal matching pursuit (OMP) algorithm is an improved implementation of matching pursuit. It has been shown that OMP can be successfully applied for compressed sensing reconstruction [180]. Once again, at every iteration an element is picked from the dictionary that best approximates the residual. However, the original function is fitted to all the already selected dictionary elements via least squares or projecting the function orthogonally onto all the selected dictionary atoms. This is also the reason it is called orthogonal matching pursuit. The OMP algorithm implementation used in this work is created by Stephen Becker [218] and it is available for MatLab in the Mathworks website. The Compressive Sampling matching pursuit (CoSaMP) algorithm is also briefly used [219], which is also based on OMP. The implementation used in chapter 4 [cross ref] is available also in [218]. 


\section{References}

1] I. E. Agency, "Technology Roadmap: Solar Photovoltaic Energy," 2014.

[2] C. Zheng and D. M. Kammen, "An innovation-focused roadmap for a sustainable global photovoltaic industry," Energy Policy, vol. 67, pp. 159-169, 2014.

[3] P. Y. Gan and Z. Li, "Quantitative study on long term global solar photovoltaic market," Renew. Sustain. Energy Rev., vol. 46, pp. 88-99, 2015.

[4] K. Yoshikawa, H. Kawasaki, W. Yoshida, T. Irie, K. Konishi, K. Nakano, T. Uto, D. Adachi, M. Kanematsu, H. Uzu, and K. Yamamoto, "Silicon heterojunction solar cell with interdigitated back contacts for a photoconversion efficiency over 26\%," Nat. Energy, vol. 2, no. 5, p. 17032, Mar. 2017.

[5] Fraunhofer Ise, "Multicrystalline Silicon Solar Cell with 21.9 Percent Efficiency: Fraunhofer ISE Again Holds World Record," $2017 . \quad$ [Online]. Available: https://www.ise.fraunhofer.de/en/press-media/press-releases/2017/multicrystalline-siliconsolar-cell-with-21-point-9-percent-efficiency-fraunhofer-ise-again-holds-world-record.html. [Accessed: 16-Jun-2017].

[6] P. Jackson, R. Wuerz, D. Hariskos, E. Lotter, W. Witte, and M. Powalla, "Effects of heavy alkali elements in $\mathrm{Cu}(\mathrm{In}, \mathrm{Ga}) \mathrm{Se2}$ solar cells with efficiencies up to $22.6 \%$," Phys. Status Solidi - Rapid Res. Lett., vol. 10, no. 8, pp. 583-586, 2016.

[7] First Solar, "First Solar Achieves yet Another Cell Conversion Efficiency World Record," First Solar Media, 2016.2 [Online]. Available: http://investor.firstsolar.com/releasedetail.cfm?ReleaselD=956479. [Accessed: 16-Jun-2017].

[8] W. Shockley and H. J. Queisser, "Detailed balance limit of efficiency of p-n junction solar cells," J. Appl. Phys., vol. 32, no. 3, pp. 510-519, 1961.

[9] T. Fuyuki, H. Kondo, T. Yamazaki, Y. Takahashi, and Y. Uraoka, "Photographic surveying of minority carrier diffusion length in polycrystalline silicon solar cells by electroluminescence," Appl. Phys. Lett., vol. 86, no. 26, pp. 1-3, 2005.

[10] T. Trupke, B. Mitchell, J. W. Weber, W. McMillan, R. a. Bardos, and R. Kroeze, "Photoluminescence imaging for photovoltaic applications," Energy Procedia, vol. 15, no. 2011, pp. 135-146, 2012.

[11] O. Breitenstein, J. P. Rakotoniaina, and M. H. Al Rifai, "Quantitative evaluation of shunts in solar cells by lock-in thermography," Prog. Photovoltaics Res. Appl., vol. 11, no. 8, pp. 515-526, Dec. 2003.

[12] J. Carstensen, G. Popkirov, J. Bahr, and H. Föll, "CELLO: an advanced LBIC measurement technique for solar cell local characterization," Sol. Energy Mater. Sol. Cells, vol. 76, no. 4, pp. 599-611, Apr. 2003.

[13] M. Padilla, B. Michl, B. Thaidigsmann, W. Warta, and M. C. Schubert, "Short-circuit current 
density mapping for solar cells," Sol. Energy Mater. Sol. Cells, vol. 120, pp. 282-288, Jan. 2014.

[14] D. Donoho, "Compressed sensing," IEEE Trans. Inf. Theory, vol. 52, no. 4, pp. 1289-1306, Apr. 2006.

[15] E. J. Candes, J. Romberg, and T. Tao, "Robust uncertainty principles: exact signal reconstruction from highly incomplete frequency information," IEEE Trans. Inf. Theory, vol. 52, no. 2, pp. 489509, Feb. 2006.

[16] M. Lustig, D. Donoho, and J. M. Pauly, "Sparse MRI: The application of compressed sensing for rapid MR imaging," Magn. Reson. Med., vol. 58, no. 6, pp. 1182-1195, Dec. 2007.

[17] L. J. Hornbeck, "The DMDTM Projection Display Chip: A MEMS-Based Technology," MRS Bull., vol. 26, no. 4, pp. 325-327, 2001.

[18] Xiaofeng Wu, M. Bliss, A. Sinha, T. R. Betts, R. Gupta, and R. Gottschalg, "Accelerated Spatially Resolved Electrical Simulation of Photovoltaic Devices Using Photovoltaic-Oriented Nodal Analysis," IEEE Trans. Electron Devices, vol. 62, no. 5, pp. 1390-1398, May 2015.

[19] V. V. Tyagi, N. A. A. Rahim, N. a. Rahim, and J. a L. Selvaraj, "Progress in solar PV technology: Research and achievement," Renew. Sustain. Energy Rev., vol. 20, pp. 443-461, 2013.

[20] N. G. Park, "Perovskite solar cells: An emerging photovoltaic technology," Materials Today, vol. 18, no. 2. Elsevier Ltd., pp. 65-72, 2015.

[21] P. Würfel, Physics of Solar Cells. Wiley-VCH Verlag GmbH \& Co. KGaA, 2005.

[22] G. . Smestad, Optoelectronics of solar cells, 1st ed. Washington, D.C.: SPIE-The International Society for Optical Engineering, 2002.

[23] S. Kasap, Principles of electronic materials and devices, 2nd editio. 2006.

[24] a. Goetzberger and V. U. Hoffmann, Photovoltaic solar energy generation, vol. 112. Berlin: Springer-Verlag Berlin Heidelberg, 2005.

[25] S. R. Wenham, M. A. Green, M. E. Watt, R. Corkish, and A. Sproul, Applied Photovoltaics, 2nd Editio. Earthscan, 2011.

[26] R. Sah, R. Noyce, and W. Shockley, "Carrier generation and recombination in pn junctions and pn junction characteristics," Proc. IRE, vol. 1, 1957.

[27] A. Cuevas and D. Macdonald, "Measuring and interpreting the lifetime of silicon wafers," Sol. Energy, vol. 76, no. 1-3, pp. 255-262, Jan. 2004.

[28] M. Wolf and H. Rauschenbach, "Series resistance effects on solar cell measurements," Adv. Energy Convers., vol. 3, no. 2, pp. 455-479, 1963.

[29] N. Enebish, D. Agchbayar, S. Dorjkhand, D. Baatar, and I. Ylemj, "Numerical analysis of solar cell current-voltage characteristics," Sol. Energy Mater. Sol. Cells, vol. 29, no. 3, pp. 201-208, 1993.

[30] M. Kntges, I. Kunze, S. Kajari-Schrder, X. Breitenmoser, and B. Bjørneklett, "The risk of power loss in crystalline silicon based photovoltaic modules due to micro-cracks," Sol. Energy Mater. Sol. Cells, vol. 95, no. 4, pp. 1131-1137, Apr. 2011. 
[31] P. Grunow, P. Clemens, V. Hoffmann, B. Litzenburger, and L. Podlowski, "Influence of micro cracks in multi-crystalline silicon solar cells on the reliability of PV modules," Proc. 20th EUPVSEC, WIP, Barcelona, Spain, no. June 2005, pp. 2042-2047, 2005.

[32] S. Kajari-Schröder, I. Kunze, and M. Köntges, "Criticality of cracks in PV modules," in Energy Procedia, 2012, vol. 27, pp. 658-663.

[33] A. Gabor, M. Ralli, S. Montminy, L. Alegria, C. Bordonaro, J. Woods, and L. Felton, "Soldering induced damage to thin Si solar cells and detection of cracked cells in modules," in 21st European Photovoltaic Solar Energy Conference, 2006.

[34] M. Köntges, M. Siebert, A. Morlier, R. Illing, N. Bessing, and F. Wegert, "Impact of transportation on silicon wafer-based photovoltaic modules," Prog. Photovoltaics Res. Appl., vol. 24, no. 8, pp. 1085-1095, Aug. 2016.

[35] X. F. Brun and S. N. Melkote, "Analysis of stresses and breakage of crystalline silicon wafers during handling and transport," Sol. Energy Mater. Sol. Cells, vol. 93, no. 8, pp. 1238-1247, 2009.

[36] O. Breitenstein, J. P. Rakotoniaina, M. H. Al Rifai, and M. Werner, "Shunt types in crystalline silicon solar cells," Prog. Photovoltaics Res. Appl., vol. 12, no. 7, pp. 529-538, Nov. 2004.

[37] S. Wendlandt and A. Drobisch, "Hot spot Risk analysis on silicon cell modules," 25th EU PVSEC, vol. 49, no. 30, pp. 1-5, 2010.

[38] D. Giaffreda, P. Magnone, M. Meneghini, M. Barbato, G. Meneghesso, E. Zanoni, E. Sangiorgi, and C. Fiegna, "Local shunting in multicrystalline silicon solar cells: Distributed electrical simulations and experiments," IEEE J. Photovoltaics, vol. 4, no. 1, pp. 40-47, 2014.

[39] M. Langenkamp and O. Breitenstein, "Classification of shunting mechanisms in crystalline silicon solar cells," Sol. Energy Mater. Sol. Cells, vol. 72, no. 1-4, pp. 433-440, 2002.

[40] A. Helbig, T. Kirchartz, R. Schaeffler, J. H. Werner, and U. Rau, "Quantitative electroluminescence analysis of resistive losses in $\mathrm{Cu}(\mathrm{In}, \mathrm{Ga}) \mathrm{Se} 2$ thin-film modules," Sol. Energy Mater. Sol. Cells, vol. 94, no. 6, pp. 979-984, Jun. 2010.

[41] S. Besold, U. Hoyer, J. Bachmann, T. Swonke, P. Schilinsky, R. Steim, and C. J. Brabec, "Quantitative imaging of shunts in organic photovoltaic modules using lock-in thermography," Sol. Energy Mater. Sol. Cells, vol. 124, pp. 133-137, 2014.

[42] S. Dongaonkar, J. D. Servaites, G. M. Ford, S. Loser, J. Moore, R. M. Gelfand, H. Mohseni, H. W. Hillhouse, R. Agrawal, M. a. Ratner, T. J. Marks, M. S. Lundstrom, and M. a. Alam, "Universality of non-Ohmic shunt leakage in thin-film solar cells," J. Appl. Phys., vol. 108, no. 12, 2010.

[43] H. J. Möller, C. Funke, M. Rinio, and S. Scholz, "Multicrystalline silicon for solar cells," Thin Solid Films, vol. 487, no. 1-2, pp. 179-187, Sep. 2005.

[44] T. Buonassisi, a. a. Istratov, M. D. Pickett, M. Heuer, J. P. Kalejs, G. Hahn, M. a. Marcus, B. Lai, Z. Cai, S. M. Heald, T. F. Ciszek, R. F. Clark, D. W. Cunningham, a. M. Gabor, R. Jonczyk, S. Narayanan, E. Sauar, and E. R. Weber, "Chemical natures and distributions of metal impurities 
in multicrystalline silicon materials," Prog. Photovoltaics Res. Appl., vol. 14, no. 6, pp. 512-531, 2006.

[45] M. Seibt, R. Khalil, V. Kveder, and W. Schröter, "Electronic states at dislocations and metal silicide precipitates in crystalline silicon and their role in solar cell materials," Appl. Phys. A Mater. Sci. Process., vol. 96, no. 1, pp. 235-253, 2009.

[46] J. D. Major, "Grain boundaries in CdTe thin film solar cells: a review," Semicond. Sci. Technol., vol. 31, no. 9, p. 93001, 2016.

[47] C. Li, Y. Wu, J. Poplawsky, T. J. Pennycook, N. Paudel, W. Yin, S. J. Haigh, M. P. Oxley, A. R. Lupini, M. Al-Jassim, S. J. Pennycook, and Y. Yan, "Grain-boundary-enhanced carrier collection in CdTe solar cells," Phys. Rev. Lett., vol. 112, no. 15, pp. 1-5, 2014.

[48] J. D. Major, Y. Y. Proskuryakov, K. Durose, G. Zoppi, and I. Forbes, "Control of grain size in sublimation-grown $\mathrm{CdTe}$, and the improvement in performance of devices with systematically increased grain size," Sol. Energy Mater. Sol. Cells, vol. 94, no. 6, pp. 1107-1112, 2010.

[49] D. Keller, S. Buecheler, P. Reinhard, F. Pianezzi, B. Bissig, R. Carron, F. Hage, Q. Ramasse, R. Erni, and A. N. Tiwari, "Band gap widening at random CIGS grain boundary detected by valence electron energy loss spectroscopy," Appl. Phys. Lett., vol. 109, no. 15, pp. 0-4, 2016.

[50] W. Li, Y. Ma, S. Yang, J. Gong, S. Zhang, and X. Xiao, "Nanoscopic study of the compositions, structures, and electronic properties of grain boundaries in $\mathrm{Cu}(\operatorname{InGa}) \mathrm{Se} 2$ photovoltaic thin films," Nano Energy, vol. 33, no. January, pp. 157-167, 2017.

[51] Y. Yan, W. J. Yin, Y. Wu, T. Shi, N. R. Paudel, C. Li, J. Poplawsky, Z. Wang, J. Moseley, H. Guthrey, H. Moutinho, S. J. Pennycook, and M. M. Al-Jassim, "Physics of grain boundaries in polycrystalline photovoltaic semiconductors," J. Appl. Phys., vol. 117, no. 11, 2015.

[52] A. Ndiaye, A. Charki, A. Kobi, C. M. F. Kébé, P. A. Ndiaye, and V. Sambou, "Degradations of silicon photovoltaic modules: A literature review," Sol. Energy, vol. 96, pp. 140-151, 2013.

[53] R. Khatri, S. Agarwal, I. Saha, S. K. Singh, and B. Kumar, "Study on long term reliability of photovoltaic modules and analysis of power degradation using accelerated aging tests and electroluminescence technique," in Energy Procedia, 2011, vol. 8, pp. 396-401.

[54] P. Chaturvedi, B. Hoex, and T. M. Walsh, "Broken metal fingers in silicon wafer solar cells and PV modules," in Solar Energy Materials and Solar Cells, 2013, vol. 108, pp. 78-81.

[55] I. Visoly-Fisher, K. D. Dobson, J. Nair, E. Bezalel, G. Hodes, and D. Cahen, "Factors affecting the stability of $\mathrm{CdTe} / \mathrm{CdS}$ solar cells deduced from stress tests at elevated temperature," Adv. Funct. Mater., vol. 13, no. 4, pp. 289-299, 2003.

[56] E. Voroshazi, B. Verreet, T. Aernouts, and P. Heremans, "Long-term operational lifetime and degradation analysis of P3HT:PCBM photovoltaic cells," Sol. Energy Mater. Sol. Cells, vol. 95, no. 5, pp. 1303-1307, 2011.

[57] Y. Han, S. Meyer, Y. Dkhissi, K. Weber, J. M. Pringle, U. Bach, L. Spiccia, and Y.-B. Cheng, "Degradation observations of encapsulated planar $\mathrm{CH} 3 \mathrm{NH} 3 \mathrm{Pbl} 3$ perovskite solar cells at high 
temperatures and humidity," J. Mater. Chem. A, vol. 3, no. 15, pp. 8139-8147, 2015.

[58] T. Trupke, R. Bardos, and M. Abbott, "Progress with luminescence imaging for the characterisation of silicon wafers and solar cells," Proceeding ..., no. September, pp. 3-7, 2007.

[59] J. Bauer, O. Breitenstein, and J. Wagner, "Lock-in thermography: a versatile tool for failure analysis of solar cells," Electron. Device Fail. Anal., vol. 3, pp. 6-12, 2009.

[60] T. Kirchartz, A. Helbig, B. E. Pieters, and U. Rau, "Electroluminescence Analysis of Solar Cells and Solar Modules," in Advanced Characterization Techniques for Thin Film Solar Cells, D. AbouRas, T. Kirchartz, and U. Rau, Eds. Weinheim: Wiley-VCH Verlag GmbH \& Co. KGaA, 2011, pp. 61-80.

[61] B. Michl, M. Padilla, I. Geisemeyer, S. T. Haag, F. Schindler, M. C. Schubert, and W. Warta, "Imaging techniques for quantitative silicon material and solar cell analysis," IEEE J. Photovoltaics, vol. 4, no. 6, pp. 1502-1510, 2014.

[62] M. Kasemann, W. Kwapil, M. C. Schubert, H. Habenicht, B. Walter, M. The, S. Kontermann, S. Rein, O. Breitenstein, J. Bauer, A. Lotnyk, B. Michl, H. Nagel, A. Schutt, J. Carstensen, H. Foll, T. Trupke, Y. Augarten, H. Kampwerth, R. a Bardos, S. Pingel, J. Berghold, W. Warta, and S. . Glunz, "Spatially resolved silicon solar cell characterization using infrared imaging methods," 33rd IEEE Photovolt. Spec. Conf., no. July 2016, pp. 1-7, 2008.

[63] F. Goucher, G. Pearson, M. Sparks, G. Teal, and W. Shockley, "Theory and Experiment for a Germanium p-n Junction," Phys. Rev., vol. 81, no. 4, pp. 637-638, 1951.

[64] C. Hu and D. Clifford, "DETERMINATION OF DIfFUSION LENGTH AND SURFACE," Solid State Electron., vol. 21, no. 2, pp. 965-968, 1978.

[65] J. D. Zook, "Effects of grain boundaries in polycrystalline solar cells," Appl. Phys. Lett., vol. 37, no. 2, p. $223,1980$.

[66] B. L. Sopori and A. Baghdadi, "Some investigations on the influence of defects/grain boundaries on photovoltaic mechanisms in polycrystalline silicon films," Sol. Cells, vol. 1, no. 3, pp. 237250, 1980.

[67] J. Gee, "The effect of parasitic absorption losses on light trapping in thin silicon solar cells," ... Conf. 1988., Conf. Rec. ..., pp. 549-554, 1988.

[68] J. Isenberg, O. Bartels, and W. Warta, "Separation of bulk diffusion length and rear surface recombination velocity in SR-LBIC mappings," Photovolt. Spec. Conf. 2002. Conf. Rec. TwentyNinth IEEE, no. 2, pp. 328-331, 2002.

[69] D. Sontag, G. Hahn, P. Geiger, P. Fath, and E. Bucher, "Two-dimensional resolution of minority carrier diffusion constants in different silicon materials," Sol. Energy Mater. Sol. Cells, vol. 72, no. 1-4, pp. 533-539, Apr. 2002.

[70] P. a. Basore, "Extended spectral analysis of internal quantum efficiency," Conf. Rec. Twenty Third IEEE Photovolt. Spec. Conf. - 1993 (Cat. No.93CH3283-9), pp. 147-152, 1993.

[71] M. Spiegel and B. Fischer, "Separation of bulk diffusion length and back surface recombination 
velocity by improved IQE-analysis," Photovolt. Spec. Conf. 2000. Conf. Rec. Twenty-Eighth IEEE, pp. 311-314, 2000.

[72] W. S. M. Brooks, S. J. C. Irvine, and V. Barrioz, "High-resolution laser beam induced current measurements on Cd0.9Zn0.1S/ CdTe solar cells," Energy Procedia, vol. 10, no. 0, pp. 232-237, Jan. 2011.

[73] F. J. Vorster and E. E. van Dyk, "High saturation solar light beam induced current scanning of solar cells.," Rev. Sci. Instrum., vol. 78, no. 1, p. 13904, 2007.

[74] T. Dennis, J. B. Schlager, and K. A. Bertness, "A novel solar simulator based on a supercontinuum laser for solar cell device and materials characterization," IEEE J. Photovoltaics, vol. 4, no. 4, pp. 1119-1127, 2014.

[75] W. Shockley and W. R. Jr, "Statistics of the recombinations of holes and electrons," Phys. Rev., no. 46, 1952.

[76] M. Stemmer, "Mapping of the local minority carrier diffusion length in silicon wafers," Appl. Surf. Sci., vol. 63, pp. 213-217, 1993.

[77] T. Yoshida, "Analysis of Photocurrent in Screen-Printed CdS/CdTe Solar Cells," J. Electrochem. Soc., vol. 139, no. 8, p. 2353, 1992.

[78] P. Vorasayan, T. R. Betts, and R. Gottschalg, "Limited laser beam induced current measurements: a tool for analysing integrated photovoltaic modules," Meas. Sci. Technol., vol. 22, no. 8, p. 85702, Aug. 2011.

[79] P. Vorasayan, T. R. Betts, A. N. Tiwari, and R. Gottschalg, "Multi-laser LBIC system for thin film PV module characterisation," Sol. Energy Mater. Sol. Cells, vol. 93, no. 6-7, pp. 917-921, Jun. 2009.

[80] J. Marek, "Light-beam-induced current characterization of grain boundaries," J. Appl. Phys., vol. 55, no. 2, p. 318, 1984.

[81] H. Takakura, K. Fujimoto, K. Okuda, C. Coluzza, and Y. Hamakawa, "Spectroscopic laser scanning analysis of photo-induced current on a-Si solar cells," Jpn. J. Appl. Phys., vol. 22, no. 4, pp. 569574, 1983.

[82] W. D. Sawyer, "An improved method of light-beam-induced current characterization of grain boundaries," J. Appl. Phys., vol. 59, no. 7, p. 2361, 1986.

[83] S. Damaskinos, "An improved laser scanning technique for evaluation of solar cells: Application to CulnSe 2/(CuZn) S devices," Sol. Cells, vol. 26, pp. 151-158, 1989.

[84] M. Breitwieser, F. D. Heinz, A. Büchler, M. Kasemann, J. Schön, W. Warta, and M. C. Schubert, "Analysis of solar cell cross sections with micro-light beam induced current ( $\mu \mathrm{LBIC})$, " Sol. Energy Mater. Sol. Cells, vol. 131, pp. 124-128, Dec. 2014.

[85] J. Hiltner and J. Sites, "High resolution laser stepping measurements on polycrystalline solar cells," 16th Eur. Photovolt. Sol. ..., no. May, 2000. 
[86] M. Rinio, H. J. Müller, and M. Werner, "LBIC investigations of the lifetime degradation by extended defects in multicrystalline solar silicon," Solid State Phenom., vol. 63-64, pp. 115$122,1998$.

[87] C. H. Seager, "The determination of grain-boundary recombination rates by scanned spot excitation methods," J. Appl. Phys., vol. 53, no. 8, p. 5968, 1982.

[88] M. S. Leite, M. Abashin, H. J. Lezec, A. Gianfrancesco, A. Alec, and N. B. Zhitenev, "Mapping the Local Photo-Electronic Properties of Polycrystalline Solar Cells through High Resolution LaserBeam-Induced Current Microscopy," J. Photovoltaics, vol. 4, no. 1, pp. 311-316, 2014.

[89] T. J. K. Brenner and C. R. McNeill, "Spatially resolved spectroscopic mapping of photocurrent and photoluminescence in polymer blend photovoltaic devices," J. Phys. Chem. C, vol. 115, no. 39, pp. 19364-19370, 2011.

[90] S. L. Howell, S. Padalkar, K. Yoon, Q. Li, D. D. Koleske, J. J. Wierer, G. T. Wang, and L. J. Lauhon, "Spatial mapping of efficiency of $\mathrm{GaN} / \mathrm{InGaN}$ nanowire array solar cells using scanning photocurrent microscopy," Nano Lett., vol. 13, no. 11, pp. 5123-5128, 2013.

[91] L. Lombez, D. Ory, M. Paire, and A. Delamarre, "Micrometric investigation of external quantum efficiency in microcrystalline CulnGa (S, Se) 2 solar cells," Thin Solid Films, vol. 565, pp. 32-36, 2014.

[92] R. M. Geisthardt and J. R. Sites, "Nonuniformity characterization of cdte solar cells using LBIC," IEEE J. Photovoltaics, vol. 4, no. 4, pp. 1114-1118, 2014.

[93] R. M. Geisthardt and J. R. Sites, "Light-beam-induced-current characterization of CdTe solar cells," in 2014 IEEE 40th Photovoltaic Specialist Conference, PVSC 2014, 2014, pp. 1848-1851.

[94] P. Engelhart and N. Harder, "RISE: $21.5 \%$ efficient back junction silicon solar cell with laser technology as a key processing tool," Photovolt. Energy Conversion, Conf. Rec. 2006 IEEE 4th World Conf., pp. 900-904, 2006.

[95] F. Castaño, D. Morecroft, M. Cascant, H. Yuste, M. Lamers, A. Mewe, I. Romijn, E. Bende, Y. Komatsu, A. Weeber, and I. Cesar, "Industrially feasible> 19\% efficiency IBC cells for pilot line processing," Photovolt. Spec. Conf. (PVSC), 2011 37th IEEE, pp. 1038-1042, 2011.

[96] F. Clement, M. Menkoe, T. Kubera, C. Harmel, R. Hoenig, W. Wolke, H. Wirth, D. Biro, and R. Preu, "Industrially feasible multi-crystalline metal wrap through (MWT) silicon solar cells exceeding 16\% efficiency," Sol. Energy Mater. Sol. Cells, vol. 93, no. 6-7, pp. 1051-1055, Jun. 2009.

[97] M. Deceglie and H. Emmer, "Scanning Laser-Beam-Induced Current Measurements of Lateral Transport Near-Junction Defects in Silicon Heterojunction Solar Cells," IEEE J. Photovoltaics, vol. 4, no. 1, pp. 154-159, Jan. 2014.

[98] N. Kwarikunda, E. van Dyk, and F. Vorster, "Application of LBIC measurements for characterisation of triple junction solar cells," Phys. B Condens. ..., pp. 1-4, 2013.

[99] Q. Chen and Y. Zhang, "The reversal of the laser-beam-induced-current contrast with varying 
illumination density in a Cu2ZnSnSe4 thin-film solar cell," Appl. Phys. Lett., vol. 103, no. 24, p. 242104, 2013.

[100] J. M. Kroon, M. M. Wienk, W. J. H. Verhees, and J. C. Hummelen, "Accurate efficiency determination and stability studies of conjugated polymer/fullerene solar cells," Thin Solid Films, vol. 403-404, pp. 223-228, Feb. 2002.

[101] F. C. Krebs, R. Søndergaard, and M. Jørgensen, "Printed metal back electrodes for R2R fabricated polymer solar cells studied using the LBIC technique," Sol. Energy Mater. Sol. Cells, vol. 95, no. 5, pp. 1348-1353, May 2011.

[102] C. Peters, I. Sachs-Quintana, J. Kastrop, S. Beaupre, M. Leclerc, and D. McGehee, "High efficiency polymer solar cells with long operating lifetimes," Adv. Energy Mater., vol. 1, pp. 491-494, 2011.

[103] M. Bokalič and M. Topič, "Light Beam Characterisation of LBIC Apparatus and Selected Complementary Applications," Proc. 46th Int. Conf. Microelectron. Devices Mater., no. October 2016, pp. 233-237, 2010.

[104] F. J. Navas, R. Alcántara, C. Fernández-Lorenzo, and J. Martín, "A methodology for improving laser beam induced current images of dye sensitized solar cells.," Rev. Sci. Instrum., vol. 80, no. 6, p. 63102, Jun. 2009.

[105] J. Navas, R. Alc?ntara, C. Fern?ndez-Lorenzo, and J. Mart?n-Calleja, "Evaluation of decay photocurrent measurements in dye-sensitized solar cells: Application to laser beam-induced current technique," Int. J. Energy Res., vol. 36, no. 2, pp. 193-203, Feb. 2012.

[106] V. Sivakov, G. Andrä, A. Gawlik, and A. Berger, "Silicon nanowire-based solar cells on glass: synthesis, optical properties, and cell parameters," Nanoletters, vol. 9, no. 4, pp. 1549-1554, 2009.

[107] M. Bliss, G. Koutsourakis, T. R. Betts, and R. Gottschalg, "Development of a Solar Cell Spectral Response Mapping System using Multi-LBIC Excitation Multi-LBIC local SR system First Measurements," in PVSAT-12 Proceedings, 2016, pp. 55-58.

[108] K. Bedrich, M. Bliss, T. R. Betts, and R. Gottschalg, "Evaluation of Uncertainty in Electroluminescence Imaging of PV Devices due to Optical and Perspective Distortions," in Proceedings of the 31st Photovoltaic Solar Energy Conference, Hamburg, 2015, p. to be published.

[109] M. Bliss, X. Wu, K. G. Bedrich, J. W. Bowers, T. R. Betts, and R. Gottschalg, "Spatially and spectrally resolved electroluminescence measurement system for photovoltaic characterisation," IET Renew. Power Gener., vol. 9, no. 5, pp. 446-452, 2015.

[110] K. Bothe, P. Pohl, and J. Schmidt, "Electroluminescence imaging as an in-line characterisation tool for solar cell production," 21st Eur. ..., no. September, pp. 597-600, 2006.

[111] a. G. Aberle, S. R. Wenham, and M. a. Green, "A new method for accurate measurements of the lumped series resistance of solar cells," in Conference Record of the Twenty Third IEEE Photovoltaic Specialists Conference - 1993 (Cat. No.93CH3283-9), 1993, pp. 133-139. 
[112] C. Schinke, D. Hinken, K. Bothe, C. Ulzhöfer, A. Milsted, J. Schmidt, and R. Brendel, "Determination of the Collection Diffusion Length by Electroluminescence Imaging," Energy Procedia, vol. 8, no. April, pp. 147-152, Jan. 2011.

[113] P. Würfel, T. Trupke, T. Puzzer, E. Schäffer, W. Warta, and S. W. Glunz, "Diffusion lengths of silicon solar cells from luminescence images," J. Appl. Phys., vol. 101, no. 12, p. 123110, 2007.

[114] J. a. Giesecke, M. Kasemann, and W. Warta, "Determination of local minority carrier diffusion lengths in crystalline silicon from luminescence images," J. Appl. Phys., vol. 106, no. 1, p. 14907, 2009.

[115] T. Potthoff, K. Bothe, U. Eitner, D. Hinken, and M. Königes, "Detection of the voltage distribution in photovoltaic modules by electroluminescence imaging," Prog. Photovoltaics Res. Appl., vol. 18, no. 2, pp. 100-106, Mar. 2010.

[116] B. Li, A. Stokes, and D. M. J. Doble, "Two Dimensional Mapping of Electrical Properties of PV Modules using Electroluminescence," in 26th European PVSEC, 2011, vol. 26, pp. 3285-3289.

[117] B. Li, A. Stokes, and D. M. J. Doble, "Evaluation of two-dimensional electrical properties of photovoltaic modules using bias-dependent electroluminescence," Prog. Photovolt Res. Appl., vol. 20, no. 2012, pp. 936-944, 2012.

[118] J. Haunschild, M. Glatthaar, M. Kasemann, S. Rein, and E. R. Weber, "Fast series resistance imaging for silicon solar cells using electroluminescence," Phys. Status Solidi - Rapid Res. Lett., vol. 3, no. 7-8, pp. 227-229, 2009.

[119] D. Hinken, K. Ramspeck, K. Bothe, B. Fischer, and R. Brendel, "Series resistance imaging of solar cells by voltage dependent electroluminescence," Appl. Phys. Lett., vol. 91, no. 18, pp. 6-9, 2007.

[120] K. Brecl, M. Bokalic, and M. Topic, "Examination of Photovoltaic Silicon Module Degradation Under High-Voltage Bias and Damp Heat by Electroluminescence," J. Sol. Energy Eng., vol. 139, no. 3, p. 31011, 2017.

[121] M. Bokalič, B. E. Pieters, A. Gerber, U. Rau, and M. Topič, "Bandgap imaging in $\mathrm{Cu}(\mathrm{In}, \mathrm{Ga}) \mathrm{Se} 2$ photovoltaic modules by electroluminescence," Prog. Photovoltaics Res. Appl., vol. 25, no. 2, pp. 184-191, Feb. 2017.

[122] M. Bokalič, J. Raguse, J. R. Sites, and M. Topič, "Analysis of electroluminescence images in small-area circular CdTe solar cells," J. Appl. Phys., vol. 114, no. 12, 2013.

[123] J. M. Raguse and J. R. Sites, "Correlation of Electroluminescence with Open-Circuit voltage from thin-films CdTe Solar Cells," IEEE J. Photovoltaics, vol. 5, no. 4, pp. 1175-1178, 2015.

[124] Y. Augarten, A. Wrigley, U. Rau, and B. E. Pieters, "Calculation of the TCO sheet resistance in thin film modules using electroluminescence imaging," Conf. Rec. IEEE Photovolt. Spec. Conf., vol. 2016-Novem, no. 4, pp. 1527-1531, 2016.

[125] A. Gerber, V. Huhn, T. M. H. Tran, M. Siegloch, Y. Augarten, B. E. Pieters, and U. Rau, “Advanced large area characterization of thin-film solar modules by electroluminescence and 
thermography imaging techniques," Sol. Energy Mater. Sol. Cells, vol. 135, pp. 35-42, 2015.

[126] M. Okano, M. Endo, A. Wakamiya, M. Yoshita, H. Akiyama, and Y. Kanemitsu, "Degradation mechanism of perovskite $\mathrm{CH} 3 \mathrm{NH} 3 \mathrm{Pbl} 3$ diode devices studied by electroluminescence and photoluminescence imaging spectroscopy," Appl. Phys. Express, vol. 8, no. 10, 2015.

[127] Z. Hameiri, A. Mahboubi Soufiani, M. K. Juhl, L. Jiang, F. Huang, Y.-B. Cheng, H. Kampwerth, J. W. Weber, M. A. Green, and T. Trupke, "Photoluminescence and electroluminescence imaging of perovskite solar cells," Prog. Photovoltaics Res. Appl., vol. 23, no. 12, pp. 1697-1705, Dec. 2015.

[128] I. Tarasov, S. Ostapenko, and J. P. Kalejs, "Defect monitoring using scanning photoluminescence spectroscopy in \nmulticrystalline silicon solar cell," Conf. Rec. Twenty-Eighth IEEE Photovolt. Spec. Conf. - 2000 (Cat. No.00CH37036), vol. 15, pp. 840-848, 2000.

[129] M. Tajima, Z. Li, S. Sumie, H. Hashizume, and A. Ogura, "Comparison of Silicon-on-Insulator Wafer Mappings between Photoluminescence Intensity and Microwave Photoconductivity Decay Lifetime," Japanese J. Appl. Physics, Part 1 Regul. Pap. Short Notes Rev. Pap., vol. 43, no. 2, pp. 432-438, 2004.

[130] K. Bothe, G. H. Bauer, and T. Unold, "Spatially resolved photoluminescence measurements on $\mathrm{Cu}(\mathrm{In}, \mathrm{Ga}) \mathrm{Se} 2$ thin films," in Thin Solid Films, 2002, vol. 403-404, pp. 453-456.

[131] T. Trupke and R. Bardos, "Photoluminescence imaging of silicon wafers," Appl. Phys. Lett., vol. 89, no. 4, p. 44107, 2006.

[132] K. Lauer, a. Laades, H. Übensee, H. Metzner, and a. Lawerenz, "Detailed analysis of the microwave-detected photoconductance decay in crystalline silicon," J. Appl. Phys., vol. 104, no. 10, p. 104503, 2008.

[133] J. a. Giesecke, M. C. Schubert, B. Michl, F. Schindler, and W. Warta, "Minority carrier lifetime imaging of silicon wafers calibrated by quasi-steady-state photoluminescence," Sol. Energy Mater. Sol. Cells, vol. 95, no. 3, pp. 1011-1018, Mar. 2011.

[134] J. Haunschild, M. Glatthaar, M. Demant, J. Nievendick, M. Motzko, S. Rein, and E. R. Weber, "Quality control of as-cut multicrystalline silicon wafers using photoluminescence imaging for solar cell production," Sol. Energy Mater. Sol. Cells, vol. 94, no. 12, pp. 2007-2012, Dec. 2010.

[135] A. Liu, Y. C. Fan, and D. Macdonald, "Interstitial iron concentrations across multicrystalline silicon wafers via photoluminescence imaging," Prog. Photovoltaics Res. Appl., vol. 19, no. 6, pp. 649-657, 2011.

[136] D. MacDonald, J. Tan, and T. Trupke, "Imaging interstitial iron concentrations in boron-doped crystalline silicon using photoluminescence," J. Appl. Phys., vol. 103, no. 7, pp. 1-8, 2008.

[137] T. Trupke, E. Pink, R. a. Bardos, and M. D. Abbott, "Spatially resolved series resistance of silicon solar cells obtained from luminescence imaging," Appl. Phys. Lett., vol. 90, no. 9, pp. 1-4, 2007.

[138] H. Kampwerth, T. Trupke, J. W. Weber, and Y. Augarten, "Advanced luminescence based effective series resistance imaging of silicon solar cells," Appl. Phys. Lett., vol. 93, no. 20, pp. 
91-94, 2008.

[139] C. Shen, H. Kampwerth, M. Green, T. Trupke, J. Carstensen, and A. Schütt, "Spatially resolved photoluminescence imaging of essential silicon solar cell parameters and comparison with CELLO measurements," Sol. Energy Mater. Sol. Cells, vol. 109, pp. 77-81, 2013.

[140] R. A. Sinton and T. Trupke, "Limitations on dynamic excess carrier lifetime calibration methods," Prog. Photovoltaics Res. Appl., vol. 20, no. 2, pp. 246-249, 2012.

[141] P. Kuo and T. Ahmed, "Phase-locked image acquisition in thermography," 1988 Robot. ..., vol. D, 1989.

[142] O. Breitenstein, J. Rakotoniaina, and Et.al., "Lock-in thermography-A universal tool for local analysis of solar cells," Eur. Photovolt. Sol. Energy Conf., no. June, pp. 590-593, 2005.

[143] O. Breitenstein and H. Straube, "Lock-in thermography investigation of solar modules," Proc. 26th Eur. PVSEC, pp. 1451-1453, 2011.

[144] M. Kaes, S. Seren, T. Pernau, and G. Hahn, "Light-modulated lock-in thermography for photosensitive pn-structures and solar cells," Prog. Photovoltaics Res. Appl., vol. 12, no. 5, pp. 355-363, 2004.

[145] O. Breitenstein, J. P. Rakotoniaina, A. S. H. van der Heide, and J. Carstensen, "Series resistance imaging in solar cells by lock-in thermography," Prog. Photovoltaics Res. Appl., vol. 13, no. 8 , pp. 645-660, Dec. 2005.

[146] A. A. S. H. van der Heide, J. H. Bultman, J. Hoornstra and andW. C. S. Schonecker, G. P.Wyers, "Locating losses due to contact resistance, shunts and recombination by potential mapping with the Corescan method," in Proceedings of 12th NRELWorkshop on Crystalline Silicon Solar CellMaterials and Processes, 2002, no. August, pp. 117-125.

[147] A. S. H. Van der Heide, J. H. Bultman, J. Hoornstra, and A. Schönecker, "Error diagnosis and optimisation of c-Si solar cell processing using contact resistances determined with the Corescanner," Sol. Energy Mater. Sol. Cells, vol. 74, no. 1-4, pp. 43-50, Oct. 2002.

[148] K. Drabczyk, G. Kulesza-Matlak, a. Drygała, M. Szindler, and M. Lipiński, "Electroluminescence imaging for determining the influence of metallization parameters for solar cell metal contacts," Sol. Energy, vol. 126, pp. 14-21, 2016.

[149] H. J. Leamy, "Charge collection scanning electron microscopy," J. Appl. Phys., vol. 53, no. 6, 1982.

[150] J. Chen, T. Sekiguchi, S. Nara, and D. Yang, "The characterization of high quality multicrystalline silicon by the electron beam induced current method," J. Phys. Condens. Matter, vol. 16, no. 2, pp. S211-S216, 2004.

[151] H. P. Yoon, P. M. Haney, D. Ruzmetov, H. Xu, M. S. Leite, B. H. Hamadani, a. A. Talin, and N. B. Zhitenev, "Local electrical characterization of cadmium telluride solar cells using low-energy electron beam," Sol. Energy Mater. Sol. Cells, vol. 117, pp. 499-504, 2013.

[152] P. Reuter, T. Rath, A. Fischereder, G. Trimmel, and P. Hadley, "Electron Beam-Induced Current 
(EBIC) in solution-processed solar cells," Scanning, vol. 33, no. 1, pp. 1-6, 2011.

[153] M. Kaseman, W. Kwapil, B. Walter, J. Giesecke, B. Michl, M. The, J.-M. Wagner, J. Bauer, A. Schutt, J. Carstensen, S. Kluska, F. Granek, H. Kampwerth, P. Gundel, M. C. Schubert, R. a Bardos, H. Foll, H. Nagel, P. Würfel, T. Trupke, O. Breitenstein, M. Hermle, W. Warta, and S. W. Glunz, "Progress in Silicon Solar Cell Characterization With Infrared Imaging Methods," 23rd Eur. Photovolt. Sol. Energy Conf., no. September, pp. 1-5, 2008.

[154] M. Kasemann, M. C. Schubert, M. The, M. Köber, M. Hermle, and W. Warta, "Comparison of luminescence imaging and illuminated lock-in thermography on silicon solar cells," Appl. Phys. Lett., vol. 89, no. 22, pp. 10-13, 2006.

[155] M. Israil, S. a. Anwar, and M. Z. Abdullah, "Automatic detection of micro-crack in solar wafers and cells: a review," Trans. Inst. Meas. Control, vol. 35, no. 5, pp. 606-618, Oct. 2012.

[156] E. J. Candès, J. K. Romberg, and T. Tao, "Stable signal recovery from incomplete and inaccurate measurements," Commun. Pure Appl. Math., vol. 59, no. 8, pp. 1207-1223, 2006.

[157] A. Jerri, "The Shannon sampling theorem - Its various extensions and applications: A tutorial review," Proc. IEEE, vol. 65, no. 11, 1977.

[158] L. Hornbeck, "Spatial Light Modualtor and Method," US Pat. 5,583,688, 1987.

[159] J. B. Sampsell, "Digital micromirror device and its application to projection displays," J. Vac. Sci. Technol. B Microelectron. Nanom. Struct., vol. 12, no. 6, p. 3242, 1994.

[160] D. W. Monk and R. O. Gale, "The digital micromirror device for projection display," Microelectron. Eng., vol. 27, no. 1-4, pp. 489-493, 1995.

[161] L. Hornbeck, “Multi-level digital micromirror device," US Pat. 5,583,688, 1996.

[162] M. Douglass, "DMD reliability: a MEMS success story," Proc. Soc. Photo-Optical Instrum. Eng., vol. 4980, pp. 1-11, 2003.

[163] M. R. Douglass, "Lifetime estimates and unique failure mechanisms of the Digital Micromirror Device (DMD)," 1998 IEEE Int. Reliab. Phys. Symp. Proceedings. 36th Annu., no. Dmd, pp. 9-16, 1998.

[164] A. B. Sontheimer and D. J. Mehrl, "Effects of operating conditions on DMD hinge memory lifetime," in IEEE International Reliability Physics Symposium Proceedings, 2003. 41st Annual., 2003, pp. 473-477.

[165] R. Gupta and O. Breitenstein, "Digital micromirror device application for inline characterization of solar cells by tomographic light beam-induced current imaging," Proc. SPIE, vol. 6616, pp. 66160-1-66160-9, 2007.

[166] J.-K. Yoo, S. K. Kim, D.-H. Lee, and S.-N. Park, "Spatial uniformity inspection apparatus for solar cells using a projection display.," Appl. Opt., vol. 51, no. 20, pp. 4563-8, 2012.

[167] J. P. Rice, S. W. Brown, D. W. Allen, H. W. Yoon, M. Litorja, and J. C. Hwang, "Hyperspectral image projector applications," Spie Moems-Mems, vol. 8254, p. 82540R, 2012. 
[168] A. Y. Fong, "Application of digital micromirror devices for spectral-response characterization of solar cells and photovoltaics," in Emerging Digital Micromirror Device Based Systems and Applications II, 2010, vol. 7596, p. 75960I-75960I-8.

[169] T. Missbach, C. Karcher, and G. Siefer, "Frequency-division-multiplex-based quantum efficiency determination of solar cells," IEEE J. Photovoltaics, vol. 6, no. 1, pp. 266-271, 2016.

[170] E. J. Candes and M. Wakin, "An Introduction To Compressive Sampling," IEEE Signal Process. Mag., vol. 25, no. 2, pp. 21-30, 2008.

[171] Y. Eldar and G. Kutyniok, Compressed sensing: theory and applications. Cambridge: Cambridge University Press, 2012.

[172] V. Abolghasemi, "On optimization of the measurement matrix for compressive sensing," EUSIPCO Aalborg, Denmark, pp. 427-431, 2010.

[173] E. Candes and T. Tao, "Decoding by linear programming," Inf. Theory, IEEE Trans., vol. 51, no. 12, pp. 4203-4215, 2005.

[174] R. G. Baraniuk, "Compressive sensing," IEEE Signal Process. Mag., vol. 24, no. 4, pp. 1-9, 2007.

[175] E. Candès and J. Romberg, "Sparsity and incoherence in compressive sampling," Inverse Probl., vol. 23, no. 3, pp. 969-985, Jun. 2007.

[176] L. Gan, T. Do, and T. Tran, "Fast compressive imaging using scrambled block Hadamard ensemble," in European Signal Processing Conference, 2008, pp. 2-6.

[177] Z. He, T. Ogawa, and M. Haseyama, "The simplest measurement matrix for compressed sensing of natural images," Image Process. (ICIP), 2010, pp. 4301-4304, 2010.

[178] E. Van Den Berg and M. P. Friedlander, "Probing the Pareto frontier for basis pursuit solutions," SIAM J. Sci. Comput., vol. 31, no. 2, pp. 890-912, 2008.

[179] E. Candes and T. Tao, "The Dantzig selector: Statistical estimation when $\mathrm{p}$ is much larger than n," Ann. Stat., vol. 35, no. 6, pp. 2313-2351, 2007.

[180] J. a. Tropp and A. C. Gilbert, "Signal recovery from random measurements via orthogonal matching pursuit," IEEE Trans. Inf. Theory, vol. 53, no. 12, pp. 4655-4666, 2007.

[181] D. Needell and J. a. Tropp, "CoSaMP: Iterative signal recovery from incomplete and inaccurate samples," Appl. Comput. Harmon. Anal., vol. 26, no. 3, pp. 301-321, 2009.

[182] M. F. Duarte, M. A. Davenport, D. Takhar, J. N. Laska, T. Sun, K. F. Kelly, and R. G. Baraniuk, "Single-Pixel Imaging via Compressive Sampling," IEEE Signal Process. Mag., vol. 25, no. 2, pp. 83-91, 2008.

[183] Jianwei Ma, "Single-Pixel Remote Sensing," IEEE Geosci. Remote Sens. Lett., vol. 6, no. 2, pp. 199-203, Apr. 2009.

[184] J. M. Lustig, Michael and Donoho, David L and Santos, Juan M and Pauly, "Compressed sensing MRI," Signal Process. Mag. IEEE, vol. 25, no. 2, pp. 72-82, 2008.

[185] C. G. Graff and E. Y. Sidky, "Compressive sensing in medical imaging," Appl. Opt., vol. 54, no. 8, 
p. C23, 2015.

[186] M. a. Herman and T. Strohmer, "High-resolution radar via compressed sensing," IEEE Trans. Signal Process., vol. 57, no. 6, pp. 2275-2284, 2009.

[187] M. Tello Alonso, P. Lopez-Dekker, and J. J. Mallorqui, “A Novel Strategy for Radar Imaging Based on Compressive Sensing," IEEE Trans. Geosci. Remote Sens., vol. 48, no. 12, pp. 4285-4295, 2010.

[188] A. C. Gurbuz, J. H. McClellan, and W. R. Scott, "Compressive sensing for subsurface imaging using ground penetrating radar," Signal Processing, vol. 89, no. 10, pp. 1959-1972, 2009.

[189] V. Studer, J. Bobin, M. Chahid, H. S. Mousavi, E. Candes, and M. Dahan, "Compressive fluorescence microscopy for biological and hyperspectral imaging.," Proc. Natl. Acad. Sci. USA, vol. 109, no. 26, pp. E1679-87, 2012.

[190] B. M. Davis, A. J. Hemphill, D. Cebeci Maltaş, M. a Zipper, P. Wang, and D. Ben-Amotz, "Multivariate Hyperspectral Raman Imaging Using Compressive Detection," Anal. Chem., vol. 83, no. 13, pp. 5086-5092, Jul. 2011.

[191] Y. August, C. Vachman, Y. Rivenson, and A. Stern, "Compressive hyperspectral imaging by random separable projections in both the spatial and the spectral domains.," Appl. Opt., vol. 52, no. 10, pp. D46-54, 2013.

[192] D. G. Carreno and H. A. Fuentes, "Transmittance analysis in coded aperture compressive Raman spectroscopy imaging," in Symposium of Signals, Images and Artificial Vision - 2013, STSIVA 2013, 2013, pp. 0-4.

[193] M. M. Marim, M. Atlan, E. D. Angelini, and J.-C. Olivo-Marin, "Compressed sensing for digital holographic microscopy," in 2010 IEEE International Symposium on Biomedical Imaging: From Nano to Macro, 2010, no. 1, pp. 684-687.

[194] P. Ye, J. L. Paredes, G. R. Arce, Y. Wu, C. Chen, and D. W. Prather, "Compressive confocal microscopy," in 2009 IEEE International Conference on Acoustics, Speech and Signal Processing, 2009, vol. 7210, pp. 429-432.

[195] J. Ma, "Compressed sensing for surface characterization and metrology," IEEE Trans. Instrum. Meas., vol. 59, no. 6, pp. 1600-1615, 2010.

[196] R. Leary, Z. Saghi, P. a. Midgley, and D. J. Holland, "Compressed sensing electron tomography," Ultramicroscopy, vol. 131, pp. 70-91, 2013.

[197] S. Andersson and L. Pao, "Non-raster sampling in atomic force microscopy: A compressed sensing approach," Am. Control Conf., no. 1, pp. 2485-2490, 2012.

[198] D. a. Redfern, E. P. G. Smith, C. a. Musca, J. M. Dell, and L. Faraone, "Interpretation of current flow in photodiode structures using laser beam-induced current for characterization and diagnostics," IEEE Trans. Electron Devices, vol. 53, no. 1, pp. 23-31, 2006.

[199] X. Wu, M. Bliss, A. Sinha, T. Betts, R. Gupta, and R. Gottschalg, "Distributed electrical network modelling approach for spatially resolved characterisation of photovoltaic modules," IET 
Renew. Power Gener., vol. 8, no. 5, pp. 459-466, Jul. 2014.

[200] X. Wu, "Fast spatially-resolved electrical modelling and quantitative characterisation of photovoltaic devices," Loughborough University, 2015.

[201] J. R. Treichler, M. a Davenport, J. N. Laska, and R. G. Baraniuk, "Dynamic range and compressive sensing acquisition receivers," Proc Def. Appl. Signal Process. DASP, no. July, 2011.

[202] J. P. Rice, J. E. Neira, M. Kehoe, and R. Swanson, "DMD diffraction measurements to support design of projectors for test and evaluation of multispectral and hyperspectral imaging sensors," Emerg. Digit. Micromirror Device Based Syst. Appl. Proc. SPIE, vol. 7210, p. 72100D, 2009.

[203] P. Ye, J. L. Paredes, Y. Wu, C. Chen, G. R. Arce, and D. W. Prather, "Compressive confocal microscopy: 3D reconstruction algorithms," in Proc. SPIE, 2009, vol. 7210, p. 72100G-72100G12.

[204] R. Y. R. Yang, S. C. S. Cheng, W. Y. W. Yang, and Y. C. Y. Chen, "Robust and Accurate Surface Measurement Using Structured Light," IEEE Trans. Instrum. Meas., vol. 57, no. 6, pp. 1275$1280,2008$.

[205] K. Li, "High-power laser phosphor light source with liquid cooling for digital cinema applications," Proc. SPIE 9005, Adv. Disp. Technol. IV, vol. 9005, p. 900507, 2014.

[206] K. Li, "LED Array with Recycling for High-Power Projector Applications," SID Symp. Dig. Tech. Pap., vol. 42, no. 1, pp. 323-325, Jun. 2011.

[207] "Flagship laser cinema projectors - Barco." [Online]. Available: http://www.barco.com/en/Products/Projectors/Cinema-projectors/Flagship-laser-cinemaprojectors. [Accessed: 01-Feb-2017].

[208] B. D. Silverstein, A. F. Kurtz, J. R. Bietry, and G. E. Nothhard, "25 . 4 : A Laser-Based Digital Cinema Projector Polarization Management," in SID 11 DIGEST, 2011, pp. 326-329.

[209] G. B. B. Alers, J. Zhou, C. Deline, P. Hacke, and S. R. R. Kurtz, "Degradation of individual cells in a module measured with differential IV analysis," Prog. Photovoltaics Res. Appl., vol. 19, no. 8, pp. 977-982, 2011.

[210] Y. S. Kim, S. M. Kang, B. Johnston, and R. Winston, "A novel method to extract the series resistances of individual cells in a photovoltaic module," Sol. Energy Mater. Sol. Cells, vol. 115, pp. 21-28, 2013.

[211] E. Candes, M. Rudelson, T. Tao, and R. Vershynin, "Error correction via linear programming," 46th Annu. IEEE Symp. Found. Comput. Sci., pp. 668-681, 2005.

[212] S. S. Chen, D. L. Donoho, and M. a Saunders, "Atomic Decomposition by Basis Pursuit," SIAM Rev. c Soc. Ind. Appl. Math., vol. 43, no. 1, pp. 129-159, 2001.

[213] S. Boyd and L. Vandenberghe, Convex Optimization, 1st ed., vol. 25, no. 3. New York: Cambridge University Press, 2010.

[214] "I1-Magic." [Online]. Available: https://statweb.stanford.edu/ candes/I1magic/. [Accessed: 
13-Dec-2016].

[215] E. Van Den Berg and M. P. Friedlander, "SPGL1: A solver for large-scale sparse reconstruction." [Online]. Available: http://www.cs.ubc.ca/ mpf/spgl1/citing.html. [Accessed: 13-Dec-2016].

[216] F. Alizadeh and D. Goldfarb, "Second-order cone programming," Math. Program. Ser. B, vol. 95, no. 1, pp. 3-51, 2003.

[217] M. S. Lobo, L. Vandenberghe, S. Boyd, and H. Lebret, "Applications of second-order cone programming," Linear Algebra Appl., vol. 284, no. 1-3, pp. 193-228, 1998.

[218] S. Becker, "CoSaMP and OMP for sparse recovery." [Online]. Available: https://uk.mathworks.com/matlabcentral/fileexchange/32402-cosamp-and-omp-for-sparserecovery/content/OMP.m. [Accessed: 12-Dec-2016].

[219] D. Needell and J. a. Tropp, "CoSaMP: Iterative signal recovery from incomplete and inaccurate samples," Appl. Comput. Harmon. Anal., vol. 26, no. 3, pp. 301-321, 2009. 


\title{
Publications and achievements
}

\author{
Journal publications
}

G. Koutsourakis, M. Cashmore, S. R. G. Hall, M. Bliss, T. R. Betts, and R. Gottschalg, “Compressed Sensing Current Mapping Spatial Characterization of Photovoltaic Devices," IEEE J. Photovoltaics, vol. 7, no. 2, pp. 486-492, Mar. 2017.

M. T. Cashmore, G. Koutsourakis, R. Gottschalg, and S. R. G. Hall, "Optical technique for photovoltaic spatial current response measurements using compressive sensing and random binary projections," J. Photonics Energy, vol. 6, no. 2, p. 25508, 2016.

S. R. G. Hall, M. Cashmore, J. Blackburn, G. Koutsourakis, and R. Gottschalg, "Compressive Current Response Mapping of Photovoltaic Devices Using MEMS Mirror Arrays," IEEE Trans. Instrum. Meas., vol. 65, no. 8, pp. 1945-1950, 2016.

\section{Conference Publications}

Oral presentations delivered

M. Bliss, B. V Mihaylov, G. Koutsourakis, T. R. Betts, and R. Gottschalg, "Uncertainty Contributions in Photocurrent Linearity Measurements of PV Devices using a Flash Solar Simulator," in PVSAT-13, 2017.

G. Koutsourakis, M. Cashmore, M. Bliss, S. R. G. Hall, T. R. Betts, and R. Gottschalg, "Compressed Sensing Current Mapping Methods for PV Characterisation," in IEEE 43nd Photovoltaic Spec. Conf., Portland, 2016.

G. Koutsourakis, M. Bliss, S. R. G. Hall, T. R. Betts, and R. Gottschalg, "Compressed Sensing Current Mapping of PV Devices Using a DLP Projector," in PVSAT-12 Proceedings, 2015. 
G. Koutsourakis, X. Wu, M. Cashmore, S. Hall, M. Bliss, T. R. Betts, and R. Gottschalg, "Fast Current Mapping Of Photovoltaic Devices By Compressive Sampling," in 31th EUPVSEC, 2015, pp. 29-34.

G. Koutsourakis, M. Cashmore, S. R. G. Hall, M. Bliss, T. R. Betts, and R. Gottschalg, "Towards Current Mapping of Photovoltaic Devices by Compressed Imaging," in PVSAT-11 proceedings, 2015, pp. 29-32.

Posters

G. Koutsourakis, S. R. G. Hall, M. Bliss, T. R. Betts, and R. Gottschalg, "A Simple Optical Setup for Current Mapping of Small Area PV Devices Using Different Sampling Strategies," in PVSAT13, 2017.

Contributions

V. Tsai, G. Koutsourakis, M. Bliss, T. R. Betts and R. Gottschalg, "Evaluation of Charge Carrier Lifetime of Thin Film Solar Cells using Transient Photovoltage Decay Measurements," in PVSAT-13.

M. Bliss, G. Koutsourakis, T. R. Betts, and R. Gottschalg, "Development of a Solar Cell Spectral Response Mapping System using Multi-LBIC Excitation Multi-LBIC local SR system First Measurements," in PVSAT-12 Proceedings, 2016, pp. 55-58.

Presentations in Workshops and Technical meetings delivered

G. Koutsourakis, M. Cashmore, S. R.G. Hall, M. Bliss, T. R Betts and R. Gottschalg "Current Mapping of Small Area PV devices using a Digital Micromirror Device Optical Setup" SuperSolar Winter Technical Meeting, 2016 
G. Koutsourakis, M. Cashmore, S. R.G. Hall, M. Bliss, T. R Betts and R. Gottschalg "Compressed Sensing Current Mapping for Spatial Characterisation of Photovoltaic Devices" National Physical Laboratory Post Graduate Institute Workshop, 2016

\section{Supervised Theses}

"Use of a Simulation to Determine I-V Characteristics of Individual Cells in a Fully Encapsulated Photovoltaic Module" C. Birkinshaw, MSc Thesis, Renewable Energy Systems Technology MSc, Loughborough University

"Novel Method for Evaluating the Series Resistance of Individual Cells in an encapsulated PV Module by Means of a DLP Projection System" J.D. Silva, MSc Thesis, Renewable Energy Systems Technology MSc, Loughborough University

\section{Awards}

Student prize award of EUPVSEC 2015: G. Koutsourakis, X. Wu, M. Cashmore, S. Hall, M. Bliss, T. R. Betts, and R. Gottschalg, "Fast Current Mapping of Photovoltaic Devices by Compressive Sampling," in 31th EUPVSEC, 2015.

Best paper award of PVSAT-11: G. Koutsourakis, M. Cashmore, S. R. G. Hall, M. Bliss, T. R. Betts, and R. Gottschalg, "Towards Current Mapping of Photovoltaic Devices by Compressed Imaging," in PVSAT-11 proceedings, 2015, pp. 29-32. 